\title{
The Strong Perfect Graph Theorem
}

\author{
Maria Chudnovsky \\ Princeton University, Princeton NJ 08544 \\ Neil Robertson ${ }^{1}$ \\ Ohio State University, Columbus, Ohio 43210 \\ Paul Seymour ${ }^{2}$ \\ Princeton University, Princeton NJ 08544 \\ Robin Thomas ${ }^{3}$ \\ Georgia Institute of Technology, Atlanta, GA 30332
}

June 20, 2002; revised July 19, 2005

${ }_{1}^{1}$ Supported by ONR grant N00014-01-1-0608, NSF grant DMS-0071096, and AIM.
${ }^{2}$ Supported by ONR grants N00014-97-1-0512 and N00014-01-1-0608, and NSF grant DMS-0070912.
${ }^{3}$ Supported by ONR grant N00014-01-1-0608, NSF grants DMS-9970514 and DMS-0200595, and AIM. 


\begin{abstract}
A graph $G$ is perfect if for every induced subgraph $H$, the chromatic number of $H$ equals the size of the largest complete subgraph of $H$, and $G$ is Berge if no induced subgraph of $G$ is an odd cycle of length at least five or the complement of one.

The "strong perfect graph conjecture" (Berge, 1961) asserts that a graph is perfect if and only if it is Berge. A stronger conjecture was made recently by Conforti, Cornuéjols and Vušković that every Berge graph either falls into one of a few basic classes, or admits one of a few kinds of separation (designed so that a minimum counterexample to Berge's conjecture cannot have either of these properties).

In this paper we prove both these conjectures.
\end{abstract}




\section{Introduction}

We begin with definitions of some of the terms we use which may be nonstandard. All graphs in this paper are finite and simple. The complement $\bar{G}$ of a graph $G$ has the same vertex set as $G$, and distinct vertices $u, v$ are adjacent in $\bar{G}$ just when they are not adjacent in $G$. A hole of $G$ is an induced subgraph of $G$ which is a cycle of length at least 4 . An antihole of $G$ is an induced subgraph of $G$ whose complement is a hole in $\bar{G}$. A graph $G$ is Berge if every hole and antihole of $G$ has even length.

A clique in $G$ is a subset $X$ of $V(G)$ such that every two members of $X$ are adjacent. A graph $G$ is perfect if for every induced subgraph $H$ of $G$, the chromatic number of $H$ equals the size of the largest clique of $H$. The study of perfect graphs was initiated by Claude Berge, partly motivated by a problem from information theory (finding the "Shannon capacity" of a graph — it lies between the size of the largest clique and the chromatic number, and so for a perfect graph it equals both). In particular, in 1961 Berge [1] proposed two celebrated conjectures about perfect graphs. Since the second implies the first, they were known as the "weak" and "strong" perfect graph conjectures respectively, although both are now theorems, the following:

\subsection{The complement of every perfect graph is perfect.}

\subsection{A graph is perfect if and only if it is Berge.}

The first was proved by Lovász [16] in 1972. The second, the strong perfect graph conjecture, received a great deal of attention over the past 40 years, but remained open until now, and is the main theorem of this paper.

It is easy to see that every perfect graph is Berge, and so to prove 1.2 it remains to prove the converse. By a minimum imperfect graph we mean a counterexample to 1.2 with as few vertices as possible (in particular, any such graph is Berge and not perfect). Much of the published work on 1.2 falls into two classes: proving that the theorem holds for graphs with some particular graph excluded as an induced subgraph, and investigating the structure of minimum imperfect graphs. For the latter, linear programming methods have been particularly useful; there are rich connections between perfect graphs and linear and integer programming (see [5, 20] for example).

But a third approach has been developing in the perfect graph community over a number of years; the attempt to show that every Berge graph either belongs to some well-understood basic class of (perfect) graphs, or admits some feature that a minimum imperfect graph cannot admit. Such a result would therefore prove that no minimum imperfect graph exists, and consequently prove 1.2. Our main result is of this type, and our first goal is to state it.

Thus, let us be more precise, and we start with two definitions. We say that $G$ is a double split graph if $V(G)$ can be partioned into four sets $\left\{a_{1}, \ldots, a_{m}\right\},\left\{b_{1}, \ldots, b_{m}\right\},\left\{c_{1}, \ldots, c_{n}\right\},\left\{d_{1}, \ldots, d_{n}\right\}$ for some $m, n \geq 2$, such that:

- $a_{i}$ is adjacent to $b_{i}$ for $1 \leq i \leq m$, and $c_{j}$ is nonadjacent to $d_{j}$ for $1 \leq j \leq n$

- there are no edges between $\left\{a_{i}, b_{i}\right\}$ and $\left\{a_{i^{\prime}}, b_{i^{\prime}}\right\}$ for $1 \leq i<i^{\prime} \leq m$, and all four edges between $\left\{c_{j}, d_{j}\right\}$ and $\left\{c_{j^{\prime}}, d_{j^{\prime}}\right\}$ for $1 \leq j<j^{\prime} \leq n$

- there are exactly two edges between $\left\{a_{i}, b_{i}\right\}$ and $\left\{c_{j}, d_{j}\right\}$ for $1 \leq i \leq m$ and $1 \leq j \leq n$, and these two edges have no common end. 
(The name is because such a graph can be obtained from what is called a "split graph" by doubling each vertex). The line graph $L(G)$ of a graph $G$ has vertex set the set $E(G)$ of edges of $G$, and $e, f \in E(G)$ are adjacent in $L(G)$ if they share an end in $G$. Let us say a graph $G$ is basic if either $G$ or $\bar{G}$ is bipartite or is the line graph of a bipartite graph, or is a double split graph. (Note that if $G$ is a double split graph then so is $\bar{G}$.) It is easy to see that all basic graphs are perfect. (For bipartite graphs it is trivial; for line graphs of bipartite graphs it is a theorem of König [15]; for their complements it follows from Lovász' theorem 1.1, although originally these were separate theorems of König; and for double split graphs we leave it to the reader.)

Now we turn to the various kinds of "features" that we will prove exist in every Berge graph that is not basic. They are all decompositions of one kind or another, so henceforth we call them that. If $X \subseteq V(G)$ we denote the subgraph of $G$ induced on $X$ by $G \mid X$. First, a special case of the "2-join" due to Cornuéjols and Cunningham [13] - a proper 2-join in $G$ is a partition $\left(X_{1}, X_{2}\right)$ of $V(G)$ such that there exist disjoint nonempty $A_{i}, B_{i} \subseteq X_{i}(i=1,2)$ satisfying:

- every vertex of $A_{1}$ is adjacent to every vertex of $A_{2}$, and every vertex of $B_{1}$ is adjacent to every vertex of $B_{2}$,

- there are no other edges between $X_{1}$ and $X_{2}$,

- for $i=1,2$, every component of $G \mid X_{i}$ meets both $A_{i}$ and $B_{i}$, and

- for $i=1,2$, if $\left|A_{i}\right|=\left|B_{i}\right|=1$ and $G \mid X_{i}$ is a path joining the members of $A_{i}$ and $B_{i}$, then it has odd length $\geq 3$.

(Thanks to Kristina Vušković for pointing out that we could include the "odd length" condition above with no change to the proof.)

If $X \subseteq V(G)$ and $v \in V(G)$, we say $v$ is $X$-complete if $v$ is adjacent to every vertex in $X$ (and consequently $v \notin X)$, and $v$ is $X$-anticomplete if $v$ has no neighbours in $X$. If $X, Y \subseteq V(G)$ are disjoint, we say $X$ is complete to $Y$ (or the pair $(X, Y)$ is complete) if every vertex in $X$ is $Y$-complete; and being anticomplete to $Y$ is defined similarly. Our second decomposition is a slight variation of the "homogeneous pair" of Chvátal and Sbihi [7] — a proper homogeneous pair in $G$ is a pair of disjoint nonempty subsets $(A, B)$ of $V(G)$, such that, if $A_{1}, A_{2}$ respectively denote the sets of all $A$-complete vertices and all $A$-anticomplete vertices in $V(G)$, and $B_{1}, B_{2}$ are defined similarly, then:

- $A_{1} \cup A_{2}=B_{1} \cup B_{2}=V(G) \backslash(A \cup B)$ (and in particular, every vertex in $A$ has a neighbour in $B$ and a nonneighbour in $B$, and vice versa)

- the four sets $A_{1} \cap B_{1}, A_{1} \cap B_{2}, A_{2} \cap B_{1}, A_{2} \cap B_{2}$ are all nonempty.

A path in $G$ is an induced subgraph of $G$ which is non-null, connected, not a cycle, and in which every vertex has degree $\leq 2$ (this definition is highly nonstandard, and we apologise, but it avoids writing "induced" about 600 times), and an antipath is an induced subgraph whose complement is a path. The length of a path is the number of edges in it (and the length of an antipath is the number of edges in its complement). We therefore recognize paths and antipaths of length 0 . If $P$ is a path, $P^{*}$ denotes the set of internal vertices of $P$, called the interior of $P$; and similarly for antipaths. Let $A, B$ be disjoint subsets of $V(G)$. We say the pair $(A, B)$ is balanced if there is no odd path between nonadjacent vertices in $B$ with interior in $A$, and there is no odd antipath between adjacent vertices 
in $A$ with interior in $B$. A set $X \subseteq V(G)$ is connected if $G \mid X$ is connected (so $\emptyset$ is connected); and anticonnected if $\bar{G} \mid X$ is connected.

The third kind of decomposition we use is due to Chvátal [6] - a skew partition in $G$ is a partition $(A, B)$ of $V(G)$ such that $A$ is not connected and $B$ is not anticonnected. Despite their elegance, skew partitions pose a difficulty that the other two decompositions do not, for it has not been shown that a minimum imperfect graph cannot admit a skew partition; indeed, this is a well-known open question, first raised by Chvátal [6], the so-called "skew partition conjecture". We get around it by confining ourselves to balanced skew partitions, which do not present this difficulty. (Another difficulty posed by skew partitions is that they are not really "decompositions" in the sense of being the inverse of a composition operation, but that does not matter for our purposes.)

We shall prove the following (the proof is the contents of sections $2-24$ ).

1.3 For every Berge graph $G$, either $G$ is basic, or one of $G, \bar{G}$ admits a proper 2-join, or $G$ admits a proper homogeneous pair, or $G$ admits a balanced skew partition.

There is in fact only one place in the entire proof that we use the homogeneous pair outcome (in the proof of 13.4), and it is natural to ask if homogeneous pairs are really needed. In fact they can be eliminated; one of us (Chudnovsky) showed in her PhD thesis [3, 4] that the following holds:

1.4 For every Berge graph $G$, either $G$ is basic, or one of $G, \bar{G}$ admits a proper 2-join, or $G$ admits a balanced skew partition.

But the proof of 1.4 is very long (it consists basically of reworking the proof of this paper for more general structures than graphs where the adjacency of some pairs of vertices is undecided) and cannot be given here, so in this paper we accept proper homogeneous pairs.

All nontrivial double split graphs admit skew partitions, so if we delete "balanced" from 1.3 then we no longer need to consider double split graphs as basic - four basic classes suffice. Unfortunately, nontrivial double split graphs do not admit balanced skew partitions, and general skew partitions are not good enough for the application to 1.2 , so we have to do it the way we did.

Let us prove that 1.3 implies 1.2. For that, we need one lemma, the following. (A maximal connected subset of a nonempty set $A \subseteq V(G)$ is called a component of $A$, and a maximal anticonnected subset is called an anticomponent of $A$.) The lemma following is related to results of [14] that were used by Roussel and Rubio in their proof [23] of 2.1. Indeed, lemma 2.2 of [14] has a similar proof, and one could use that lemma to make this proof a little shorter.

1.5 If $G$ is a minimum imperfect graph, then $G$ admits no balanced skew partition.

Proof. Suppose that $(A, B)$ is a balanced skew partition of $G$, and let $B_{1}$ be an anticomponent of $B$. Let $G^{\prime}$ be the graph obtained from $G$ by adding a new vertex $z$ with neighbour set $B_{1}$.

(1) $G^{\prime}$ is Berge.

For suppose not. Then in $G^{\prime}$ there is an odd hole or antihole using $z$. Suppose first that there is an odd hole, $C$ say. Then the neighbours of $z$ in $C$ (say $x, y$ ) belong to $B_{1}$, and no other vertex of $B_{1}$ is in $C$. No vertex of $B \backslash B_{1}$ is in $C$ since it would be adjacent to $x, y$ and $C$ would have length 4; so $C \backslash z$ is an odd path of $G$, with ends in $B_{1}$ and with interior in $A$, contradicting that $(A, B)$ 
is balanced. So we may assume there is no such $C$. Now assume there is an odd antihole $D$ in $G^{\prime}$, again using $z$. Then exactly two vertices of $D \backslash z$ are nonadjacent to $z$, so all the others belong to $B_{1}$. Hence in $G$ there is an odd antipath $Q$ of length $\geq 3$, with ends $x, y \notin B_{1}$ and with interior in $B_{1}$. Since both $x$ and $y$ have nonneighbours in the interior of $Q$ it follows that $x, y \notin B$; and so $x, y \in A$, again contradicting that $(A, B)$ is balanced. This proves (1).

For a subset $X$ of $V(G)$, we denote the size of the largest clique in $X$ by $\omega(X)$. Let $\omega\left(B_{1}\right)=s$, and $\omega(A \cup B)=t$. Since $G$ is minimum imperfect it cannot be $t$-coloured. Let $A_{1}, \ldots, A_{m}$ be the components of $A$.

(2) For $1 \leq i \leq m$ there is a subset $C_{i} \subseteq A_{i}$ such that $\omega\left(C_{i} \cup B_{1}\right)=s$ and

$$
\omega\left(\left(A_{i} \backslash C_{i}\right) \cup\left(B \backslash B_{1}\right)\right) \leq t-s .
$$

For let $H=G^{\prime} \mid\left(B \cup A_{i} \cup\{z\}\right)$; then $H$ is Berge, by (1). Now by [6], there are at least two vertices of $G$ not in $H$ (all the vertices in $A \backslash A_{i}$ ), and since $H$ has only one new vertex it follows that $|V(H)|<|V(G)|$. From the minimality of $|V(G)|$ we deduce that $H$ is perfect. Now a theorem of Lovász [16] shows that replicating a vertex of a perfect graph makes another perfect graph; so if we replace $z$ by a set $Z$ of $t-s$ vertices all complete to $B_{1}$ and to each other, and with no other neighbours in $A_{i} \cup B$, then the graph we make is perfect. From the construction, the largest clique in this graph has size $\leq t$, and so it is $t$-colourable. Since $Z$ is a clique of size $t-s$, we may assume that colours $1, \ldots, s$ do not occur in $Z$, and colours $s+1, \ldots, t$ do. Since $B_{1}$ is complete to $Z$, colours $s+1, \ldots, t$ do not occur in $B_{1}$, and so only colours $1, \ldots, s$ occur in $B_{1}$; and since $\omega\left(B_{1}\right)=s$, all these colours do occur in $B_{1}$. Since $B_{1}$ is complete to $B \backslash B_{1}$, none of colours $1, \ldots, s$ occur in $B \backslash B_{1}$. Let $C_{i}$ be the set of vertices $v \in A_{i}$ with colours $1, \ldots, s$. Then $C_{i} \cup B_{1}$ has been coloured using only $s$ colours, and so $\omega\left(C_{i} \cup B_{1}\right)=s$; and the remainder of $H \backslash z$ has been coloured using only colours $s+1, \ldots, t$, and so

$$
\omega\left(\left(A_{i} \backslash C_{i}\right) \cup\left(B \backslash B_{1}\right)\right) \leq t-s .
$$

This proves (2).

Now let $C=B_{1} \cup C_{1} \cup \cdots \cup C_{m}$ and $D=V(G) \backslash C$. Since there are no edges between different $A_{i}$ 's, it follows from $(2)$ that $\omega(C)=s$, and similarly $\omega(D) \leq t-s$. Since $|C|,|D|<|V(G)|$ it follows that $G|C, G| D$ are both perfect; so they are $s$-colourable and $(t-s)$-colourable, respectively. But then $G$ is $t$-colourable, a contradiction. Thus there is no such $(A, B)$. This proves 1.5.

\section{Proof of 1.2, assuming 1.3.}

Suppose that there is a minimum imperfect graph $G$. Thus $G$ is Berge and not perfect. Every basic graph is perfect, and so $G$ is not basic. It is shown in [13] that $G$ does not admit a proper 2-join. From Lovász's theorem 1.1, it follows that $\bar{G}$ is also a minimum imperfect graph, and therefore $\bar{G}$ also does not admit a proper 2-join. It is shown in [7] that $G$ does not admit a proper homogeneous pair, and $G$ does not admit a balanced skew partition by 1.5. It follows that $G$ violates 1.3, and therefore there is no such graph $G$. This proves 1.2.

There were a series of statements like 1.3 conjectured over the past twenty years (although they were mostly unpublished, and were unknown to us when we were working on 1.3.) Let us sketch the 
course of evolution, kindly furnished to us by a referee. A star cutset is a skew partition $(A, B)$ such that some vertex of $B$ is adjacent to all other vertices of $B$. An even pair means a pair of vertices $u, v$ in a graph such that every path between them has even length. It was known $[2,6,18]$ that no minimum imperfect graph admits a star cutset or an even pair, and the earlier versions of 1.3 involved these concepts. For instance, in Reed's PhD thesis [19], the following conjecture appears:

1.6 Conjecture: For every perfect graph $G$, either one of $G, \bar{G}$ is a line graph of a bipartite graph, or one of them has a star cutset or an even pair.

Reed also studied the same question for Berge graphs, and researchers at that time were considering using general skew partitions instead of star cutsets (although this would not by itself imply 1.2 , since the skew partition conjecture was still open).

A counterexample to all these versions of the conjecture was obtained in the early 1990's by Irena Rusu. At about the same time, Conforti, Cornuéjols and Rao [9] proved a statement analogous to 1.3 for the class of bipartite graphs in which every induced cycle has length a multiple of four, and their theorem involved 2-joins. Since Cornuéjols and Cunningham [13] had already proved that no minimum imperfect graph admits a 2-join, it was natural to add 2-joins to the arsenal.

At a conference in Princeton in 1993, Conforti and Cornuéjols gave a series of talks on their work; and in working sessions at the conference (particularly one in which Irena Rusu presented her counterexample), new variants of the conjecture were discussed, including the following:

\subsection{Conjecture: For every Berge graph $G$, either}

- one of $G, \bar{G}$ is a line graph of a bipartite graph, or

- one of $G, \bar{G}$ admits a 2-join, or

- $G$ admits a skew partition, or

- one of $G, \bar{G}$ has an even pair.

More recently, Conforti, Cornuéjols and Vušković [10] proposed a similar conjecture, with the "even pair" alternative replaced by "one of $G, \bar{G}$ is bipartite", although without explicitly listing a proposed set of decompositions. Our result 1.3 is essentially a version of this conjecture, except that we only accept skew partitions that are balanced (and therefore need a fifth basic class) and also we include homogeneous pairs.

How can we prove a theorem of the form of 1.3 ? There are several other theorems of this kind in graph theory - for example, [7, 10, 17, 21, 22, 24, 25] and others. All these theorems say that "every graph (or matroid) not containing an object of type X either falls into one of a few basic classes or admits a decomposition". And for each of these theorems, the proof is basically a combination of the same two methods (below, we say "graph" and "subgraph", although the objects and containment relations vary depending on the context):

- We judiciously choose an explicit X-free graph $H$ ( $X$-free means not containing a subgraph of type $\mathrm{X}$ ) that does not fall into any of the basic classes; check that it has a decomposition of the kind it is supposed to have; and show that this decomposition extends to a decomposition of every bigger X-free graph containing $H$. That proves that the theorem is true for all X-free graphs that contain $H$, so now we may focus on the X-free graphs that do not contain $H$. 
- We choose a graph $J$, in one of the basic classes and "decently-connected", whatever that means in the circumstances. Let $G$ be a bigger $X$-free graph containing $J$ that we still need to understand. Enlarge $J$ to a maximal subgraph $K$ of $G$ that is still decently-connected and belongs to the same basic class as $J$. We can assume that $K \neq G$, for otherwise $G$ satisfies the theorem. Making use of the maximality of $K$, we prove that the way the remainder of $G$ attaches to $K$ is sufficiently restricted that we can infer a decomposition of $G$. Now we may focus on the $X$-free graphs that do not contain $J$.

It turns out that these two methods can be used for Berge graphs, in just the same way. We need about twelve iterations of this process.

The paper is organized as follows. The next three sections develop tools that will be needed all through the paper. Section 2 concerns a fundamental lemma of Roussel and Rubio; we give several variations and extensions of it, and more in section 3 , of a different kind. In section 4 we develop some features of skew partitions, to make them easier to handle in the main proof, which we begin in section 5. Sections 5-8 prove that every Berge graph containing a "substantial" line graph as an induced subgraph, satisfies 1.3 ("substantial" means a line graph of a bipartite subdivision of a 3 -connected graph $J$, with some more conditions if $J=K_{4}$ ). Section 9 proves the same thing for line graphs of subdivisions of $K_{4}$ that are not "substantial" - this is where double split graphs come in. In section 10 we prove that Berge graphs containing an "even prism" satisfy 1.3. (To prove this we may assume we are looking at a Berge graph that does not contain the line graph of a subdivision of $K_{4}$, for otherwise we could apply the results of the earlier sections. The same thing happens later - at each step we may assume the current Berge graph does not contain any of the subgraphs that were handled in earlier steps.) Sections 11-13 do the same for "long odd prisms", and section 14 does the same for a subgraph we call the "double diamond". Section 15 is a break for resharpening the tools we proved in the first four sections, and in particular, here we prove Chvátal's skew partition conjecture [6], that no minimum imperfect graph admits a skew partition. (Or almost - Chvátal actually conjectured that no minimal imperfect graph admits a skew partition, and we only prove it here for minimum imperfect graphs. But that is all we need, and of course the full conjecture of Chvátal follows from 1.2.) Section 16 proves that any Berge graph containing what we call an "odd wheel" satisfies 1.3, in sections 17-23 we prove the same for wheels in general, and finally in section 24 we handle Berge graphs not containing wheels.

These steps are summarized more precisely in the next theorem, which we include now in the hope that it will be helpful to the reader, although some necessary definitions have not been given yet — for the missing definitions, the reader should see the appropriate section(s) later. Let $\mathcal{F}_{1}, \ldots, \mathcal{F}_{11}$ be the classes of Berge graphs defined as follows (each is a subclass of the previous class):

- $\mathcal{F}_{1}$ is the class of all Berge graphs $G$ such that for every bipartite subdivision $H$ of $K_{4}$, every induced subgraph of $G$ isomorphic to $L(H)$ is degenerate

- $\mathcal{F}_{2}$ is the class of all graphs $G$ such that $G, \bar{G} \in \mathcal{F}_{1}$ and no induced subgraph of $G$ is isomorphic to $L\left(K_{3,3}\right)$

- $\mathcal{F}_{3}$ is the class of all Berge graphs $G$ such that for every bipartite subdivision $H$ of $K_{4}$, no induced subgraph of $G$ or of $\bar{G}$ is isomorphic to $L(H)$

- $\mathcal{F}_{4}$ is the class of all $G \in \mathcal{F}_{3}$ such that no induced subgraph of $G$ is an even prism 
- $\mathcal{F}_{5}$ is the class of all $G \in \mathcal{F}_{3}$ such that no induced subgraph of $G$ or of $\bar{G}$ is a long prism

- $\mathcal{F}_{6}$ is the class of all $G \in \mathcal{F}_{5}$ such that no induced subgraph of $G$ is isomorphic to a double diamond

- $\mathcal{F}_{7}$ is the class of all $G \in \mathcal{F}_{6}$ such that $G$ and $\bar{G}$ do not contain odd wheels

- $\mathcal{F}_{8}$ is the class of all $G \in \mathcal{F}_{7}$ such that $G$ and $\bar{G}$ do not contain pseudowheels

- $\mathcal{F}_{9}$ is the class of all $G \in \mathcal{F}_{8}$ such that $G$ and $\bar{G}$ do not contain wheels

- $\mathcal{F}_{10}$ is the class of all $G \in \mathcal{F}_{9}$ such that, for every hole $C$ in $G$ of length $\geq 6$, no vertex of $G$ has three consecutive neighbours in $C$, and the same holds in $\bar{G}$

- $\mathcal{F}_{11}$ is the class of all $G \in \mathcal{F}_{10}$ such that every antihole in $G$ has length 4.

1.8 (The steps of the proof of 1.3)

1. For every Berge graph $G$, either $G$ is a line graph of a bipartite graph, or $G$ admits a proper 2 -join or a balanced skew partition, or $G \in \mathcal{F}_{1}$; and (consequently) either one of $G, \bar{G}$ is a line graph of a bipartite graph, or one of $G, \bar{G}$ admits a proper 2-join, or $G$ admits a balanced skew partition, or $G, \bar{G} \in \mathcal{F}_{1}$.

2. For every $G$ with $G, \bar{G} \in \mathcal{F}_{1}$, either $G=L\left(K_{3,3}\right)$, or $G$ admits a balanced skew partition, or $G \in \mathcal{F}_{2}$.

3. For every $G \in \mathcal{F}_{2}$, either $G$ is a double split graph, or one of $G, \bar{G}$ admits a proper 2-join, or $G$ admits a balanced skew partition, or $G \in \mathcal{F}_{3}$.

4. For every $G \in \mathcal{F}_{1}$, either $G$ is an even prism with $|V(G)|=9$, or $G$ admits a proper 2-join or a balanced skew partition, or $G \in \mathcal{F}_{4}$.

5. For every $G$ such that $G, \bar{G} \in \mathcal{F}_{4}$, either one of $G, \bar{G}$ admits a proper 2-join, or $G$ admits a proper homogeneous pair, or $G$ admits a balanced skew partition, or $G \in \mathcal{F}_{5}$.

6. For every $G \in \mathcal{F}_{5}$, either one of $G, \bar{G}$ admits a proper 2-join, or $G$ admits a balanced skew partition, or $G \in \mathcal{F}_{6}$.

7. For every $G \in \mathcal{F}_{6}$, either $G$ admits a balanced skew partition, or $G \in \mathcal{F}_{7}$.

8. For every $G \in \mathcal{F}_{7}$, either $G$ admits a balanced skew partition, or $G \in \mathcal{F}_{8}$.

9. For every $G \in \mathcal{F}_{8}$, either $G$ admits a balanced skew partition, or $G \in \mathcal{F}_{9}$.

10. For every $G \in \mathcal{F}_{9}$, either $G$ admits a balanced skew partition, or $G \in \mathcal{F}_{10}$.

11. For every $G \in \mathcal{F}_{10}$, either $G \in \mathcal{F}_{11}$ or $\bar{G} \in \mathcal{F}_{11}$.

12. For every $G \in \mathcal{F}_{11}$, either $G$ admits a balanced skew partition, or $G$ is complete or bipartite.

The twelve statements of 1.8 are proved in $5.1,5.2,9.6,10.6,13.4,14.3,16.3,18.7,23.2,23.4$, 23.5 , and 24.1 respectively. 


\section{The Roussel-Rubio lemma}

There is a beautiful and very powerful theorem of [23] which we use many times throughout the paper. (We proved it independently, in joint work with Carsten Thomassen, but Roussel and Rubio found it earlier.) Its main use is to show that in some respects, the common neighbours of an anticonnected set of vertices (in a Berge graph) act like or almost like the neighbours of a single vertex.

If $X \subseteq V(G)$, we say an edge $u v$ is $X$-complete if $u, v$ are both $X$-complete. Let $P$ be a path in $G$ (we remind the reader that this means $P$ is an induced subgraph which is a path), of length $\geq 2$, and let the vertices of $P$ be $p_{1}, \ldots, p_{n}$ in order. A leap for $P($ in $G)$ is a pair of nonadjacent vertices $a, b$ of $G$ such that there are exactly six edges of $G$ between $a, b$ and $V(P)$, namely $a p_{1}, a p_{2}, a p_{n}, b p_{1}, b p_{n-1}, b p_{n}$.

The Roussel-Rubio lemma (slightly reformulated for convenience) is the following.

2.1 Let $G$ be Berge, let $X \subseteq V(G)$ be anticonnected, and $P$ be a path in $G \backslash X$ with odd length, such that both ends of $P$ are $X$-complete. Then either:

1. some edge of $P$ is $X$-complete, or

2. $P$ has length $\geq 5$ and $X$ contains a leap for $P$, or

3. $P$ has length 3 and there is an odd antipath with interior in $X$, joining the internal vertices of $P$.

This has a number of corollaries that again we shall need throughout the paper, and in this section we prove some of them.

2.2 Let $G$ be Berge, let $X$ be an anticonnected subset of $V(G)$, and $P$ be a path in $G \backslash X$ with odd length, such that both ends of $P$ are $X$-complete, and no edge of $P$ is $X$-complete. Then every $X$-complete vertex of $G$ has a neighbour in $P^{*}$.

Proof. Let $v$ be $X$-complete. Certainly $P$ has length $>1$, since its ends are $X$-complete and therefore nonadjacent. Suppose first it has length $>3$. Then by 2.1, $X$ contains a leap, and so there is a path $Q$ with ends in $X$ and with $Q^{*}=P^{*}$. Then $v$ is adjacent to both ends of $Q$, and since $G \mid(V(Q) \cup\{v\})$ is not an odd hole, it follows that $v$ has a neighbour in $Q^{*}=P^{*}$, as required. Now suppose $P$ has length 3 , and let its vertices be $p_{1^{-}} \cdots-p_{4}$ in order. By 2.1 , there is an odd antipath $Q$ between $p_{2}$ and $p_{3}$ with interior in $X$. Since $Q$ cannot be completed to an odd antihole via $p_{3}-v-p_{2}$, it follows that $v$ is adjacent to one of $p_{2}, p_{3}$, as required.

Here is another easy lemma that gets used enough that it is worth stating separately.

2.3 Let $G$ be Berge, let $X \subseteq V(G)$ be anticonnected, and let $P$ be a path or hole in $G \backslash X$. Let $Q$ be a subpath of $P$ (and hence of $G$ ) with both ends $X$-complete. Then either the number of $X$-complete edges in $Q$ has the same parity as the length of $Q$, or the ends of $Q$ are the only $X$-complete vertices in $P$. In particular, if $P$ is a hole, then either there are an even number of $X$-complete edges in $P$, or there are exactly two $X$-complete vertices and they are adjacent. 
Proof. The second assertion follows from the first. For the first, we use induction on the length of $Q$. If some internal vertex of $Q$ is $X$-complete then the result follows from the inductive hypothesis, so we may assume not. If $Q$ has length 1 or even then the theorem holds, so we may assume its length is $\geq 3$ and odd. We may assume that there is an $X$-complete vertex $v$ say of $P$ that is not an end of $Q$, and therefore does not belong to $Q$; and since $P$ is a path or hole, it follows that $v$ has no neighbour in $Q^{*}$, contrary to 2.2 . This proves 2.3 .

A triangle in $G$ is a set of three vertices, mutually adjacent. We say a vertex $v$ can be linked onto a triangle $\left\{a_{1}, a_{2}, a_{3}\right\}$ (via paths $\left.P_{1}, P_{2}, P_{3}\right)$ if:

- the three paths $P_{1}, P_{2}, P_{3}$ are mutually vertex-disjoint

- for $i=1,2,3 a_{i}$ is an end of $P_{i}$

- for $1 \leq i<j \leq 3, a_{i} a_{j}$ is the unique edge of $G$ between $V\left(P_{i}\right)$ and $V\left(P_{j}\right)$

- $v$ has a neighbour in each of $P_{1}, P_{2}$ and $P_{3}$.

The following is well-known and quite useful:

2.4 Let $G$ be Berge, and suppose $v$ can be linked onto a triangle $\left\{a_{1}, a_{2}, a_{3}\right\}$. Then $v$ is adjacent to at least two of $a_{1}, a_{2}, a_{3}$.

Proof. Let $v$ be linked via paths $P_{1}, P_{2}, P_{3}$. For $1 \leq i \leq 3, v$ has a neighbour in $P_{i}$; let $P_{i}$ be the path from $v$ to $a_{i}$ with interior in $V\left(Q_{i}\right)$. At least two of $Q_{1}, Q_{2}, Q_{3}$ have lengths of the same parity, say $Q_{1}, Q_{2}$; and since $G \mid\left(V\left(Q_{1}\right) \cup V\left(Q_{2}\right)\right)$ is not an odd hole, it is a cycle of length 3 , and the claim follows.

A variant of 2.2 is sometimes useful, the following:

2.5 Let $G$ be Berge, let $X \subseteq V(G)$, and let $P$ be a path in $G \backslash X$ of odd length, with vertices be $p_{1}-\cdots-p_{n}$, such that $p_{1}, p_{n}$ are $X$-complete, and no edge of $P$ is $X$-complete. Let $v \in V(G)$ be $X$-complete. Then either $v$ is adjacent to one of $p_{1}, p_{2}$, or the only neighbour of $v$ in $P^{*}$ is $p_{n-1}$.

Proof. By 2.2, $v$ has a neighbour in $P^{*}$, and we may assume that $p_{n-1}$ is not its only such neighbour, so $v$ has a neighbour in $\left\{p_{2}, \ldots, p_{n-2}\right\}$. If $P$ has length $\leq 3$ then the result follows, so we may assume its length is at least 5 . By 2.1 , there is a leap $a, b$ for $P$ in $X$; so there is a path $a-p_{2^{-}} \cdots-p_{n-1}-b$. Now $\left\{p_{1}, p_{2}, a\right\}$ is a triangle, and $v$ can be linked onto it via the three paths $b-p_{1}, P \backslash\left\{p_{1}, p_{n-1}, p_{n}\right\}$, $a$; and so $v$ has two neighbours in the triangle, by 2.4 , and the claim follows.

2.6 If $G$ is Berge and $A, B \subseteq V(G)$ are disjoint, and $v \in V(G) \backslash(A \cup B)$, and $v$ is complete to $B$ and anticomplete to $A$, then $(A, B)$ is balanced.

The proof is clear.

2.7 Let $(A, B)$ be balanced in a Berge graph $G$. Let $C \subseteq V(G) \backslash(A \cup B)$. Then :

1. if $A$ is connected and every vertex in $B$ has a neighbour in $A$, and $A$ is anticomplete to $C$, then $(C, B)$ is balanced 
2. if $B$ is anticonnected and no vertex in $A$ is $B$-complete, and $B$ is complete to $C$, then $(A, C)$ is balanced.

Proof. The first statement follows from the second by taking complements, so it suffices to prove the second. Suppose $u, v \in A$ are adjacent and joined by an odd antipath $P$ with interior in $C$. Since $B$ is anticonnected and $u, v$ both have non-neighbours in $B$, they are also joined by an antipath $Q$ with interior in $B$, which is even since $(A, B)$ is balanced. But then $u-P-v-Q-u$ is an odd antihole, a contradiction. Now suppose there are nonadjacent $u, v \in C$, joined by an odd path $P$ with interior in $A$. Then $P$ has length $\geq 5$, since otherwise its vertices could be reordered to be an odd antipath of the kind we already handled. The ends of $P$ are $B$-complete, and no internal vertex is $B$-complete, and so $B$ contains a leap for $P$ by 2.1 ; and hence there is an odd path with ends in $B$ and interior in $A$, which is impossible since $(A, B)$ is balanced. This proves 2.7 .

We already said what we mean by linking a vertex onto a triangle, but now we do the same for an anticonnected set. We say an anticonnected set $X$ can be linked onto a triangle $\left\{a_{1}, a_{2}, a_{3}\right\}$ (via paths $\left.P_{1}, P_{2}, P_{3}\right)$ if:

- the three paths $P_{1}, P_{2}, P_{3}$ are mutually vertex-disjoint

- for $i=1,2,3 a_{i}$ is an end of $P_{i}$

- for $1 \leq i<j \leq 3, a_{i} a_{j}$ is the unique edge of $G$ between $V\left(P_{i}\right)$ and $V\left(P_{j}\right)$

- each of $P_{1}, P_{2}$ and $P_{3}$ contains an $X$-complete vertex.

There is a corresponding extension of 2.4, the following:

2.8 Let $G$ be Berge, let $X$ be an anticonnected set, and suppose $X$ can be linked onto a triangle $\left\{a_{1}, a_{2}, a_{3}\right\}$ via $P_{1}, P_{2}, P_{3}$. For $i=1,2,3$ let $P_{i}$ have ends $a_{i}$ and $b_{i}$, and let $b_{i}$ be the unique vertex of $P_{i}$ that is $X$-complete. Then either at least two of $P_{1}, P_{2}, P_{3}$ have length 0 (and hence two of $a_{1}, a_{2}, a_{3}$ are $X$-complete) or one of $P_{1}, P_{2}, P_{3}$ has length 0 and the other two have length 1 (say $P_{3}$ has length 0$)$; and in this case, every $X$-complete vertex in $G$ is adjacent to one of $a_{1}, a_{2}$.

Proof. Some two of $P_{1}, P_{2}, P_{3}$ have lengths of the same parity, say $P_{1}$ and $P_{2}$. Hence the path $Q=b_{1}-P_{1}-a_{1}-a_{2}-P_{2}-b_{2}$ (with the obvious meaning - we shall feel free to specify paths by whatever notation is most convenient) is odd, and its ends are $X$-complete, and none of its internal vertices are $X$-complete. If $Q$ has length 1 then the theorem holds, so we assume it has length $\geq 3$. By 2.2, every $X$-complete vertex has a neighbour in $Q^{*}$, and since $b_{3}$ is $X$-complete, it follows that $b_{3}=a_{3}$. Hence we may assume both $P_{1}$ and $P_{2}$ have length $\geq 1$ for otherwise the claim holds. Suppose that $Q$ has length 3 . Then $P_{1}$ and $P_{2}$ have length 1 , and the claim holds again. So we may assume (for a contradiction) that $Q$ has length $\geq 5$, and from the symmetry we may assume $P_{1}$ has length $\geq 2$. Since $b_{3}$ is not adjacent to the end $b_{1}$ of $Q$ or to its neighbour in $Q$, and yet it has at least two neighbours in $Q^{*}$ (namely $a_{1}$ and $a_{2}$ ), this contradicts 2.5 . This proves 2.8 .

As we said earlier, the main use of 2.1 is to show that the common neighbours of an anticonnected set behave in some respects like the neighbours of a single vertex. From this point of view, 2.1 itself tells us something about when there can be an odd "pseudohole", in which one "vertex" is actually an anticonnected set. We also need a version of this when there are two such vertices, the following. 
2.9 Let $G$ be Berge, and let $X, Y$ be disjoint nonempty anticonnected subsets of $V(G)$, complete to each other. Let $P$ be a path in $G \backslash(X \cup Y)$ with even length $>0$, with vertices $p_{1}, \ldots, p_{n}$ in order, such that $p_{1}$ is the unique $X$-complete vertex of $P$ and $p_{n}$ is the unique $Y$-complete vertex of $P$. Then either:

1. $P$ has length $\geq 4$ and there are nonadjacent $x_{1}, x_{2} \in X$ such that $x_{1}-p_{2}-\cdots-p_{n}-x_{2}$ is a path, or

2. $P$ has length $\geq 4$ and there are nonadjacent $y_{1}, y_{2} \in Y$ such that $y_{1}-p_{1}-\cdots-p_{n-1}-y_{2}$ is a path, or

3. $P$ has length 2 and there is an antipath $Q$ between $p_{2}$ and $p_{3}$ with interior in $X$, and an antipath $R$ between $p_{1}$ and $p_{2}$ with interior in $Y$, and exactly one of $Q, R$ has odd length.

In each case, either $\left(V\left(P \backslash p_{1}\right), X\right)$ or $\left(V\left(P \backslash p_{n}\right), Y\right)$ is not balanced.

Proof. It follows from the hypotheses that $X, Y$ and $V(P)$ are mutually disjoint. If $P$ has length 2, choose an antipath $Q$ between $p_{2}$ and $p_{3}$ with interior in $X$, and an antipath $R$ between $p_{1}$ and $p_{2}$ with interior in $Y$. Then $p_{2}-Q-p_{3}-p_{1}-R-p_{2}$ is an antihole, and so exactly one of $Q, R$ has odd length and the theorem holds. So we may assume $P$ has length $\geq 4$. We may assume that $V(G)=V(P) \cup X \cup Y$, by deleting any other vertices. Let $G^{\prime}$ be obtained from $G \backslash Y$ by adding a new vertex y with neighbour set $X \cup\left\{p_{n}\right\}$. Let $P^{\prime}$ be the path $p_{1^{-}} \cdots-p_{n^{-}} y$ of $G^{\prime}$. Then $P^{\prime}$ has odd length $\geq 5$. If $G^{\prime}$ is Berge then by 2.1 there is a leap for $P^{\prime}$ in $X$, and the result follows. So we may assume $G^{\prime}$ is not Berge.

Assume first that there is an odd hole $C$ of length $\geq 7$ in $G^{\prime}$. It necessarily uses $y$, and the neighbours of $y$ in $C$ are $Y$-complete, and no other vertices of $C \backslash y$ are $Y$-complete. Hence there is an odd path $Q$ in $G \backslash Y$ of length $\geq 5$, with both ends $Y$-complete and no internal vertices $Y$ complete. So the ends of $Q$ belong to $X \cup\left\{p_{n}\right\}$ and its interior to $V(P) \backslash\left\{p_{n}\right\}$. By $2.1 Y$ contains a leap for $Q$; so there is an odd path $R$ of length $\geq 5$ with ends $\left(y_{1}, y_{2}\right.$ say) in $Y$ and with interior in $V(P) \backslash\left\{p_{n}\right\}$. Since $R$ cannot be completed to a hole via $y_{2}-p_{n}-y_{1}$ it follows that $p_{n}$ has a neighbour in $R^{*}$, and so $p_{n-1}$ belongs to $R$. If also $p_{1}$ belongs to $R$ then the theorem holds, so we may assume it does not. Since $R$ is odd and $P$ is even it follows that $p_{2}$ also does not belong to $R$, and so $p_{1}$ has no neighbour in $R^{*}$; yet the ends of $R$ are $X$-complete and its internal vertices are not, contrary to 2.2. This completes the case when there is an odd hole in $G^{\prime}$ of length $\geq 7$.

Since an odd hole of length 5 is also an odd antihole, we may assume that there is an odd antihole in $G^{\prime}$, say $D$. Again $D$ must use $y$, and uses exactly two nonneighbours of $y$; so in $G$ there is an odd antipath $Q$ between adjacent vertices of $P \backslash p_{n}$ (say $u$ and $v$ ), and with interior in $X \cup\left\{p_{n}\right\}$. Since $u$ and $v$ are not $Y$-complete, they are also joined by an antipath $R$ with interior in $Y$, and $R$ must also be odd since its union with $Q$ is an antihole. Since $R$ cannot be completed to an antihole via $v-p_{n}-u$ it follows that $p_{n}$ is adjacent to one of $u, v$, and hence we may assume that $u=p_{n-2}$ and $v=p_{n-1}$. Since $P$ has length $\geq 4$ it follows that $u, v$ are also joined by an antipath with interior in $X$, say $S$, and again $S$ is odd since its union with $R$ is an antihole. But $S$ can be completed to an antihole via $v-p_{1}-u$, a contradiction. This proves 2.9 .

Next we need a version of 2.1 for holes. Let $C$ be a hole in $G$, and let $e=u v$ be an edge of it. A leap for $C$ (in $G$, at uv) is a leap for the path $C \backslash e$ in $G \backslash e$. A hat for $C$ (in $G$, at uv) is a vertex of $G$ adjacent to $u$ and $v$ and to no other vertex of $C$. 
2.10 Let $G$ be Berge, let $X \subseteq V(G)$ be anticonnected, let $C$ be a hole in $G \backslash X$ with length $>4$, and let $e=u v$ be an edge of $C$. Assume that $u, v$ are $X$-complete and no other vertex of $C$ is $X$-complete. Then either $X$ contains a hat for $C$ at uv, or $X$ contains a leap for $C$ at uv.

Proof. Let the vertices of $C$ be $p_{1}, \ldots, p_{n}$ in order, where $u=p_{1}$ and $v=p_{n}$. Let $G_{1}=G \mid(V(C) \cup X)$, and let $G_{2}=G_{1} \backslash e$. If $G_{2}$ is Berge, then from 2.1 applied to the path $C \backslash e$ in $G_{2}$ it follows that $X$ contains a leap for $C$ at $u v$. So we may assume that $G_{2}$ is not Berge. Consequently it has an odd hole or antihole $D$ say, and since $D$ is not an odd hole or antihole in $G_{1}$ it must use both $p_{1}$ and $p_{n}$. Suppose first that $D$ is an odd hole. Since every vertex in $X$ is adjacent to both $p_{1}$ and $p_{n}$ it follows that at most one vertex of $X$ is in $D$; and since $G_{2} \backslash X$ has no cycles, there is exactly one vertex of $X$ in $D$, say $x$. Hence $D \backslash x$ is a path of $G_{2} \backslash X$ between $p_{1}$ and $p_{n}$, and so $D \backslash x=C \backslash e$; and since $D$ is a hole of $G_{2}$ it follows that $x$ has no neighbours in $\left\{p_{2}, \ldots, p_{n-1}\right\}$, and therefore is a hat as required. Next assume that $D$ is an antihole. Since it uses both $p_{1}$ and $p_{n}$, and they are nonadjacent in $G_{2}$, it follows that they are consecutive in $D$, so the vertices of $D$ can be numbered $d_{1}, \ldots, d_{m}$ in order, where $d_{1}=p_{1}$ and $d_{m}=p_{n}$, and therefore $m \geq 5$. Consequently, both $d_{2}$ and $d_{m-1}$ are not in $X$, since they are not complete to $\left\{p_{1}, p_{n}\right\}$, and therefore $d_{1}, d_{2}, d_{m-1}, d_{m}$ are vertices of $C$. Yet $d_{1} d_{m-1}, d_{m-1} d_{2}, d_{2} d_{m}$ are edges of $G_{1}$, which is impossible since $n \geq 6$. This proves 2.10 .

There is an analogous version of 2.9 , as follows.

2.11 Let $G$ be Berge, and let $X, Y$ be disjoint nonempty anticonnected subsets of $V(G)$, complete to each other. Let $P$ be a path in $G \backslash(X \cup Y)$ with even length $\geq 4$, with vertices $p_{1}, \ldots, p_{n}$ in order, such that $p_{1}$ is the unique $X$-complete vertex of $P$, and $p_{1}, p_{n}$ are the only $Y$-complete vertices of $P$. Then either:

1. there exists $x \in X$ non-adjacent to all of $p_{2}, \ldots, p_{n}$, or

2. there are nonadjacent $x_{1}, x_{2} \in X$ such that $x_{1}-p_{2}-\cdots-p_{n}-x_{2}$ is a path.

Proof. The proof is similar to that of 2.9. We may assume $V(G)=V(P) \cup X \cup Y$. Let $G^{\prime}$ be obtained from $G \backslash Y$ by adding a new vertex y with neighbour set $X \cup\left\{p_{1}, p_{n}\right\}$. If $G^{\prime}$ is Berge then the result follows from 2.10, so we may assume $G^{\prime}$ is not Berge. Assume first that there is an odd hole $C$ of length $\geq 7$ in $G^{\prime}$. Hence there is an odd path $Q$ in $G \backslash Y$ of length $\geq 5$, with both ends $Y$-complete and no internal vertices $Y$-complete. So the ends of $Q$ belong to $X \cup\left\{p_{1}, p_{n}\right\}$ and its interior to $V\left(P^{*}\right)$. By $2.1 Y$ contains a leap for $Q$; so there is an odd path $R$ of length $\geq 5$ with ends $\left(y_{1}, y_{2}\right.$ say) in $Y$ and with interior in $V\left(P^{*}\right)$. Since $R$ is odd and $R^{*}$ is a subpath of the even path $P^{*}$, it follows that not both $p_{2}$ and $p_{n-1}$ belong to $R$; but then $R$ can be completed to an odd hole via one of $y_{2}-p_{n}-y_{1}, y_{2}-p_{1}-y_{1}$, a contradiction. This completes the case when there is an odd hole in $G^{\prime}$ of length $\geq 7$, so now we may assume that there is an odd antihole in $G^{\prime}$, say $D$. Again $D$ must use $y$, and uses exactly two nonneighbours of $y$; so in $G$ there is an odd antipath $Q$ between adjacent vertices of $P^{*}$ (say $u$ and $v$ ), and with interior in $X \cup\left\{p_{n}\right\}$. Since $u$ and $v$ are not $Y$-complete, they are also joined by an antipath $R$ with interior in $Y$, and $R$ must also be odd since its union with $Q$ is an antihole. Since one of $p_{1}, p_{n}$ is nonadjacent to both of $u, v$, we may complete $R$ to an odd antihole via one of $u-p_{1}-v, u-p_{n}-v$, a contradiction. This proves 2.11 . 


\section{Paths and antipaths meeting}

Another class of applications of 2.1 is to the situation when a long path or hole meets a long antipath or antihole. In this section we prove a collection of useful lemmas of this type. First, a neat application of 2.1 (we include this only because it is striking - in fact we do not use it at all).

3.1 Let $G$ be Berge, let $C$ be a hole in $G$, and $D$ an antihole in $G$, both of length $\geq 8$. Then $|V(C) \cap V(D)| \leq 3$.

Proof. It is easy to see that $|V(C) \cap V(D)| \leq 4$, without using that $G$ is Berge. Suppose that $|V(C) \cap V(D)|=4$; then $V(C) \cap V(D)$ is the vertex set of a 3 -edge path. Let $C$ have vertices $p_{1}, \ldots, p_{m}$ in order, and $D$ have vertices $q_{1}, \ldots, q_{n}$ in order, where $m, n \geq 8$ and $p_{1}=q_{2}, p_{2}=q_{4}, p_{3}=q_{1}, p_{4}=q_{3}$. Let $P$ be the path $p_{4}-p_{5^{-}} \cdots-p_{m}-p_{1}$, and $Q$ the antipath $q_{4^{-}} q_{5^{-}} \cdots-q_{n^{-}} q_{1}$. Let $X$ be the interior of $Q$. Then $p_{1}$ and $p_{4}$ are $X$-complete (since $D$ is an antihole), and $P$ is a path with length odd and $\geq 5$ between these two vertices. If some vertex $p_{i}$ say in the interior of $P$ is $X$-complete, then since $p_{i}$ is nonadjacent to both $p_{2}$ and $p_{3}$ we can complete $Q$ to an odd antihole via $q_{1}-p_{i}-q_{4}$, a contradiction. So by $2.1 X$ contains a leap for $P$; so there exists $i$ with $5 \leq i<n$ and a path $P^{\prime}$ joining $q_{i}$ and $q_{i+1}$ with the same interior as $P$. Since $n \geq 8$, either $i>5$ or $i+1<n$ and from the symmetry we may assume the first. But then $P^{\prime}$ can be completed to an odd hole via $q_{i+1}-p_{2}-q_{i}$, a contradiction. This proves 3.1 .

The next two lemmas are results of the same kind:

3.2 Let $p_{1}-\cdots-p_{m}$ be a path in a Berge graph $G$. Let $2 \leq s \leq m-2$, and let $p_{s}-q_{1}-\cdots-q_{n}-p_{s+1}$ be an antipath, where $n \geq 2$ is odd. Assume that each of $q_{1}, \ldots, q_{n}$ has a neighbour in $\left\{p_{1}, \ldots, p_{s-1}\right\}$ and a neighbour in $\left\{p_{s+2}, \ldots, p_{m}\right\}$. Then either:

- $s \geq 3$ and the only nonedges between $\left\{p_{s-2}, p_{s-1}, p_{s}, p_{s+1}, p_{s+2}\right\}$ and $\left\{q_{1}, \ldots, q_{n}\right\}$ are $p_{s-1} q_{n}$, $p_{s} q_{1}, p_{s+1} q_{n}$, or

- $s \leq m-3$ and the only nonedges between $\left\{p_{s-1}, p_{s}, p_{s+1}, p_{s+2}, p_{s+3}\right\}$ and $\left\{q_{1}, \ldots, q_{n}\right\}$ are $p_{s} q_{1}, p_{s+1} q_{n}, p_{s+2} q_{1}$.

Proof. The antipath $p_{s^{-}} q_{1^{-}} \cdots-q_{n}-p_{s+1}$ is even, of length $\geq 4$; all its vertices have neighbours in $\left\{p_{1}, \ldots, p_{s-1}\right\}$ except $p_{s+1}$, and they all have neighbours in $\left\{p_{s+2}, \ldots, p_{m}\right\}$ except $p_{s}$. Since the sets $\left\{p_{1}, \ldots, p_{s-1}\right\},\left\{p_{s+2}, \ldots, p_{m}\right\}$ are both connected and are anticomplete to each other, it follows from 2.9 applied in $\bar{G}$ and the symmetry that we may assume that there are adjacent vertices $u, v \in\left\{p_{1}, \ldots, p_{s-1}\right\}$ such that $u-p_{s^{-}} q_{1^{-}} \cdots-q_{n^{-}} v$ is an antipath. Since $v$ is adjacent to $p_{s}$ and to $u$ it follows that $s \geq 3, v=p_{s-1}$ and $u=p_{s-2}$. Since $p_{s-2}-p_{s^{-}} q_{1^{-}} \cdots-q_{n-1} p_{s-1}$ is an odd antipath of length $\geq 5$, and its ends are anticomplete to $\left\{p_{s+1}, \ldots, p_{m}\right\}$ and its internal vertices are not, it follows from 2.1 applied in $\bar{G}$ that there are adjacent $w, x \in\left\{p_{s+1}, \ldots, p_{m}\right\}$ such that $w-p_{s^{-}} q_{1^{-}} \cdots-q_{n^{-}} x$ is an antipath. Since $x$ is adjacent to $p_{s}$ and to $w$ it follows that $x=p_{s+1}$ and $w=p_{s+2}$. But then the theorem holds. This proves 3.2 .

3.3 Let $G$ be Berge, let $C$ be a hole in $G$ of length $\geq 6$, with vertices $p_{1}, \ldots, p_{m}$ in order, and let $Q$ be an antipath with vertices $p_{1}, q_{1}, \ldots, q_{n}, p_{2}$, with length $\geq 4$ and even. Let $z \in V(G)$, complete to $V(Q)$ and with no neighbours among $p_{3}, \ldots, p_{m}$. There is at most one vertex in $\left\{p_{3}, \ldots, p_{m}\right\}$ complete to either $\left\{q_{1}, \ldots, q_{n-1}\right\}$ or $\left\{q_{2}, \ldots, q_{n}\right\}$, and any such vertex is one of $p_{3}, p_{m}$. 
Proof. It follows that none of $q_{1}, \ldots, q_{n}$ belong to $C$, since they are all adjacent to $z$. Let $X=\left\{q_{1}, \ldots, q_{n}\right\}$, and let $Y_{1}, Y_{2}$ be the sets of vertices in $\left\{p_{3}, \ldots, p_{m}\right\}$ complete to $X \backslash\left\{q_{n}\right\}, X \backslash\left\{q_{1}\right\}$ respectively.

(1) $Y_{1} \subseteq Y_{2} \cup\left\{p_{m}\right\}$, and $Y_{2} \subseteq Y_{1} \cup\left\{p_{3}\right\}$.

For suppose some $p_{i} \in Y_{1}$, and is not in $Y_{2}$; then since the odd antipath $Q \backslash p_{2}$ cannot be completed to an odd antihole via $q_{n}-p_{i}-p_{1}$, it follows that $i=m$. This proves (1).

(2) If $Y_{1} \nsubseteq\left\{p_{m}\right\}$ then $p_{3} \in Y_{1} \cap Y_{2}$, and if $Y_{2} \nsubseteq\left\{p_{3}\right\}$ then $p_{m} \in Y_{1} \cap Y_{2}$.

For assume $Y_{1} \nsubseteq\left\{p_{m}\right\}$, and choose $i$ with $3 \leq i \leq m-1$ minimum so that $p_{i} \in Y_{1}$. By (1), $p_{i} \in Y_{2}$, so we may assume $i>3$, for otherwise the claim holds. If $i$ is odd, then the path $p_{2}-p_{3^{-}} \cdots-p_{i}$ is odd and between $X \backslash\left\{q_{n}\right\}$-complete vertices, and no internal vertex is $X \backslash\left\{q_{n}\right\}$-complete, and yet the $X \backslash\left\{q_{n}\right\}$-complete vertex $z$ does not have a neighbour in its interior, contrary to 2.2. So $i$ is even. The path $p_{i^{-}} \cdots-p_{m}-p_{1}$ is therefore odd, and has length $\geq 3$, and its ends are $X \backslash\left\{q_{1}\right\}$-complete, and the $X \backslash\left\{q_{1}\right\}$-complete vertex $z$ does not have a neighbour in its interior; so by 2.2 some vertex $v$ of its interior is in $Y_{2}$, and therefore in $Y_{1} \cap Y_{2}$ by (1). But the path $z-p_{2} \cdots-p_{i}$ is odd, and between $X$-complete vertices, and has no more such vertices in its interior, and $v$ has no neighbour in its interior, contrary to 2.2 . This proves (2).

Now not both $p_{3}, p_{m}$ are in $Y_{1} \cap Y_{2}$, for otherwise $Q$ could be completed to an odd antihole via $p_{2}-p_{m}-p_{3}-p_{1}$. Hence we may assume $p_{3} \notin Y_{1} \cap Y_{2}$, and so from (2), $Y_{1} \subseteq\left\{p_{m}\right\}$. By (1), $Y_{2} \subseteq\left\{p_{3}\right\} \cup Y_{1}$, and so $Y_{1} \cup Y_{2} \subseteq\left\{p_{3}, p_{m}\right\}$. We may therefore assume that $Y_{1} \cup Y_{2}=\left\{p_{3}, p_{m}\right\}$, for otherwise the theorem holds. In particular, $p_{3} \in Y_{2}$. If also $p_{m} \in Y_{2}$, then $p_{3}-p_{4}-\cdots-p_{m}$ is an odd path between $X \backslash\left\{q_{1}\right\}$-complete vertices, and none of its internal vertices are $X \backslash\left\{q_{1}\right\}$-complete, and yet the

$X \backslash\left\{q_{1}\right\}$-complete vertex $z$ does not have a neighbour in its interior, contrary to 2.2 . So $p_{m} \notin Y_{2}$, and so $p_{m} \in Y_{1}$; but then $p_{3}-q_{1}-q_{2^{-}} \cdots-q_{n}-p_{m}-p_{3}$ is an odd antihole, a contradiction. This proves 3.3.

\section{Skew partitions}

In the main proof (which starts in the next section), it happens quite frequently that we can identify a skew partition in $G$, and what we really want is to show that $G$ admits a balanced skew partition. In this section we prove several lemmas to facilitate that process.

4.1 Let $G$ be Berge, and suppose that $G$ admits a skew partition $(A, B)$ such that either some component of $A$ or some anticomponent of $B$ has only one vertex. Then $G$ admits a balanced skew partition.

Proof. By taking complements if necessary we may assume that for some $a_{1} \in A,\left\{a_{1}\right\}$ is a component of $A$. Let $N$ be the set of vertices of $G$ adjacent to $a_{1}$; so $N \subseteq B$. Assume first that $N$ is not anticonnected. Then $(V(G) \backslash N, N)$ is a skew partition of $G$, and it is easy to check that it is balanced, as required. So we may assume that $N$ is anticonnected. Consequently $N$ is a subset of 
some anticomponent of $B$, say $B_{1}$. Choose $b_{2} \in B \backslash B_{1}$. Then $N^{\prime}=N \cup\left\{b_{2}\right\}$ is not anticonnected, and so $\left(V(G) \backslash N^{\prime}, N^{\prime}\right)$ is a skew partition of $G$, and once again it is easily checked to be balanced. This proves 4.1 .

Let us say a skew partition $(A, B)$ of $G$ is loose if either some vertex in $B$ has no neighbour in some component of $A$, or some vertex in $A$ is complete to some anticomponent of $B$. In the main proof later in the paper, many of the skew partitions we construct are loose, and so the next lemma is very useful.

4.2 If $G$ is Berge, and admits a loose skew partition, then it admits a balanced skew partition.

Proof. Let $(A, B)$ be a loose skew partition of $G$. By taking complements if necessary, we may assume that some vertex in $B$ has no neighbour in some component of $A$. With $G$ fixed, let us choose the skew partition $(A, B)$ and a component $A_{1}$ of $A$ and an anticomponent $B_{1}$ of $B$ with $|B|-2\left|B_{1}\right|$ minimum, such that some vertex in $B_{1}$ (say $b_{1}$ ) has no neighbour in $A_{1}$. (We call this property the "optimality" of $(A, B)$.) Let the other components of $A$ be $A_{2}, \ldots, A_{m}$, and the other anticomponents of $B$ be $B_{2}, \ldots, B_{n}$. By 4.1 we may assume that no $\left|A_{i}\right|$ or $\left|B_{j}\right|=1$, and in this case we shall show that the skew partition $(A, B)$ is balanced.

(1) For $2 \leq j \leq n$, no vertex in $A$ is $B_{j}$-complete and not $B_{1}$-complete, and every vertex in $B \backslash B_{1}$ has a neighbour in $A_{1}$.

For the first claim, assume some vertex $v \in A$ is $B_{2}$-complete and not $B_{1}$-complete, say. Let $A_{1}^{\prime}=A_{1}$ if $v \notin A_{1}$, and let $A_{1}^{\prime}$ be a maximal connected subset of $A_{1} \backslash\{v\}$ otherwise. (So $A_{1}^{\prime}$ is nonempty since we assumed $\left|A_{1}\right| \geq 2$.) Let $A^{\prime}=A \backslash\{v\}$ and $B^{\prime}=B \cup\{v\}$; then $B_{2}$ is still an anticomponent of $B^{\prime}$, so $\left(A^{\prime}, B^{\prime}\right)$ is a skew partition, violating the optimality of $(A, B)$ (for since $v$ is not $B_{1}$-complete, there is an anticomponent of $B^{\prime}$ including $\left.B \cup\{v\}\right)$. For the second claim, assume that some vertex $v \in B_{2}$ say has no neighbour in $A_{1}$. Then since $\left|B_{2}\right| \geq 2$, it follows that $(A \cup\{v\}, B \backslash\{v\})$ is a skew partition of $G$, again violating the optimality of $(A, B)$. This proves (1).

By 2.6, the pair $\left(A_{1}, B_{j}\right)$ is balanced, for $2 \leq j \leq n$, since $b_{1}$ is complete to $B_{j}$ and has no neighbours in $A_{1}$. By (1) and 2.7.1, it follows that $\left(A_{i}, B_{j}\right)$ is balanced for $2 \leq i \leq m$ and $2 \leq j \leq n$. It remains to check all the pairs $\left(A_{i}, B_{1}\right)$. Let $1 \leq i \leq m$, and let $A_{i}^{\prime}$ be the set of vertices in $A_{i}$ that are not $B_{1}$-complete. By (1), no vertex in $A_{i}^{\prime}$ is $B_{2}$-complete, and $\left(A_{i}^{\prime}, B_{2}\right)$ is balanced, and hence by 2.7.2, so is $\left(A_{i}^{\prime}, B_{1}\right)$, and consequently so is $\left(A_{i}, B_{1}\right)$. This proves that $(A, B)$ is balanced, and so completes the proof of 4.2 .

4.3 Let $(A, B)$ be a skew partition of a Berge graph $G$. If either:

- there exist $u, v \in B$ joined by an odd path with interior in $A$, and joined by an even path with interior in $A$, or

- there exist $u, v \in A$ joined by an odd antipath with interior in $B$, and joined by an even antipath with interior in $B$,

then $(A, B)$ is loose and therefore $G$ admits a balanced skew partition. 
Proof. By taking complements we may assume that the first case of the theorem applies. There is an even path $P_{1}$ and an odd path $P_{2}$ joining $u, v$, both with interior in $A$. Let $A_{1}$ be the component of $A$ including the interior of $P_{1}$. Since $P_{1} \cup P_{2}$ is not a hole, it follows that $P_{2}$ also has interior in $A_{1}$. Let $A_{2}$ be another component of $A$. If $u, v$ are joined by a path with interior in $A_{2}$, then its union with one of $P_{1}, P_{2}$ would be an odd hole, a contradiction; so there is no such path. Hence one of $u, v$ has no neighbours in $A_{2}$, and hence $(A, B)$ is loose, and the theorem follows from 4.2. This proves 4.3.

If $(A, B)$ is a skew partition of $G$, and $A^{\prime}$ is a component of $A$, and $B^{\prime}$ is an anticomponent of $B$, we call the pair $\left(A^{\prime}, B^{\prime}\right)$ a path pair if there is an odd path in $G$ with ends nonadjacent vertices of $B^{\prime}$ and with interior in $A^{\prime}$; and $\left(A^{\prime}, B^{\prime}\right)$ is an antipath pair if there is an odd antipath in $G$ with ends adjacent vertices of $A^{\prime}$ and with interior in $B^{\prime}$.

4.4 Let $(A, B)$ be a skew partition of a Berge graph $G$, and let $A_{1}, \ldots, A_{m}$ be the components of $A$, and $B_{1}, \ldots, B_{n}$ the anticomponents of $B$. Then either:

- $(A, B)$ is loose or balanced, or

- $\left(A_{i}, B_{j}\right)$ is a path pair for all $i, j$ with $1 \leq i \leq m$ and $1 \leq j \leq n$, or

- $\left(A_{i}, B_{j}\right)$ is an antipath pair for all $i, j$ with $1 \leq i \leq m$ and $1 \leq j \leq n$.

Proof. We may assume $(A, B)$ is not loose and not balanced.

(1) If for some $i, j$ there is an odd path of length $\geq 5$ with ends in $B_{j}$ and interior in $A_{i}$, then the theorem holds.

For assume there is such a path for $i=j=1$ say. Let this path, $P_{1}$ say, have vertices $b_{1}-p_{1}-p_{2}-\ldots-p_{n^{-}}-b_{1}^{\prime}$, where $b_{1}, b_{1}^{\prime} \in B_{1}$ and $p_{1}, \ldots, p_{n} \in A_{1}$. Let $2 \leq j \leq n$. Then $P_{1}$ is an odd path of length $\geq 5$ between common neighbours of $B_{j}$, and no internal vertex of it is $B_{j}$-complete since $(A, B)$ is not loose. By 2.1, $B_{j}$ contains a leap; so there exist nonadjacent $b_{j}, b_{j}^{\prime} \in B_{j}$ such that $b_{j}-p_{1}-p_{2^{-}} \ldots-p_{n}-b_{j}^{\prime}$ is a path. Hence $\left(A_{1}, B_{j}\right)$ is a path pair. Now let $2 \leq i \leq m$ and $1 \leq j \leq n$. Since $(A, B)$ is not loose, $b_{j}$ and $b_{j}^{\prime}$ both have neighbours in $A_{i}$, and so there is a path $P_{2}$ say joining them with interior in $A_{i}$; it is odd by 4.3 , and so $\left(A_{i}, B_{j}\right)$ is a path pair. This proves (1).

From (1) we may assume that for all $i, j$, every odd path of length $>1$ with ends in $B_{j}$ and interior in $A_{i}$ has length 3 ; and similarly every odd antipath of length $>1$ with ends in $A_{i}$ and interior in $B_{j}$ has length 3 . Consequently, every path pair is also an antipath pair (because a path of length 3 can be reordered to be an antipath of length 3$)$. We may assume that $\left(A_{1}, B_{1}\right)$ is a path pair, and so there exist $b_{1}, b_{1}^{\prime} \in B_{1}$ and $a_{1}, a_{1}^{\prime} \in A_{1}$ such that $b_{1}-a_{1}-a_{1}^{\prime}-b_{1}^{\prime}$ is a path $P_{1}$ say. Let $2 \leq i \leq m$. Since $b_{1}$ and $b_{1}^{\prime}$ both have neighbours in $A_{i}$, they are joined by a path with interior in $A_{i}$, odd by 4.3 ; and so by (1) it has length 3 . Hence there exist $a_{i}, a_{i}^{\prime} \in A_{i}$ such that $b_{1}-a_{i}-a_{i}^{\prime}-b_{1}^{\prime}$ is a path. By the same argument in the complement, it follows that for all $1 \leq i \leq m$ and $2 \leq j \leq n$, there exist $b_{j}, b_{j}^{\prime} \in B_{j}$ such that $b_{j}-a_{i}-a_{i}^{\prime}-b_{j}^{\prime}$ is a path. So every pair $\left(A_{i}, B_{j}\right)$ is both a path and antipath pair. This proves 4.4 . 
We can reformulate the previous result in a form that is easier to apply, as follows.

4.5 Let $G$ be Berge. Suppose that there is a partition of $V(G)$ into four nonempty sets $X, Y, L, R$, such that there are no edges between $L$ and $R$, and $X$ is complete to $Y$. If either:

- some vertex in $X \cup Y$ has no neighbours in $L$ or no neighbours in $R$, or

- some vertex in $L \cup R$ is complete to $X$ or complete to $Y$, or

- $(L, Y)$ is balanced

then $G$ admits a balanced skew partition.

Proof. Certainly $(L \cup R, X \cup Y)$ is a skew partition, so by 4.2 we may assume it is not loose, and therefore neither of the first two alternative hypotheses holds. So we assume the third hypothesis holds. Let $A_{1}, \ldots, A_{m}$ be the components of $L \cup R$, and let $B_{1}, \ldots, B_{n}$ be the anticomponents of $X \cup Y$. Since $X, Y, L, R$ are all nonempty we may assume that $A_{1} \subseteq L$, and $B_{1} \subseteq X$. By hypothesis, $\left(A_{1}, B_{1}\right)$ is not a path or antipath pair, and so by 4.4 the skew partition is balanced. This proves 4.5 .

Let $(A, B)$ be a skew partition of $G$. We say that an anticonnected subset $W$ of $B$ is a kernel for the skew partition if some component of $A$ contains no $W$-complete vertex.

4.6 Let $(A, B)$ be a skew partition of a Berge graph $G$, and let $W$ be a kernel for it. Let $A_{1}$ be a component of $A$, and suppose that

- every pair of nonadjacent vertices of $W$ with neighbours in $A_{1}$ are joined by an even path with interior in $A$

- every pair of adjacent vertices of $A_{1}$ with nonneighbours in $W$ are joined by an even antipath with interior in $B$.

Then $G$ admits a balanced skew partition.

Proof. By 4.2 we may assume $(A, B)$ is not loose. Let the components of $A$ be $A_{1}, \ldots, A_{m}$, and the anticomponents of $B$ be $B_{1}, \ldots, B_{n}$.

(1) $\left(A_{i}, W\right)$ is balanced for $1 \leq i \leq m$.

For this is true by 4.3 if $i=1$, so assume $i>1$. From 4.3 there is no odd path between nonadjacent vertices of $W$ with interior in $A_{i}$. Suppose there is an odd antipath $Q$ of length $>1$, with ends in $A_{i}$ and interior in $W$. Then it has length $\geq 5$, for otherwise it can be reordered to be an odd path that we have already shown impossible. Now the ends of $Q$ have no neighbours in the connected set $A_{1}$, and its internal vertices all have neighbours in $A_{1}$; and so by 2.1 in the complement, there is a leap in the complement; that is, there is an antipath with ends in $A_{1}$ and with the same interior as $Q$, which is impossible. This proves (1).

Since $W$ is anticonnected, we may assume that $W \subseteq B_{1}$. Since (1) restores the symmetry between $A_{1}, \ldots, A_{m}$, we may assume that there is no $W$-complete vertex in $A_{1}$. By 4.4 we may 
assume $\left(A_{1}, B_{2}\right)$ is a path or antipath pair. Suppose first that it is an antipath pair. Then there is an odd antipath $Q_{1}$ of length $\geq 3$ with ends in $A_{1}$ and interior in $B_{2}$. Since its ends both have nonneighbours in $W$, its ends are also joined by an antipath $Q_{2}$ with interior in $W$, odd by 4.3 , contrary to (1). So there is no such $Q_{1}$. Hence there is an odd path $P$ with ends in $B_{2}$ and interior in $A_{1}$, necessarily of length $\geq 5$ (since we already did the antipath case). Since the interior of $P$ contains no $W$-complete vertex, 2.1 implies that $W$ contains a leap; and so there is a path with ends in $W$ with the same interior as $P$, a contradiction. This proves 4.6.

\section{Small attachments to a line graph}

We come now to the first of the major steps of the proof. Suppose that $G$ is Berge, and contains as an induced subgraph a substantial line graph $L(H)$. Then in general, $G$ itself can only be basic by being a line graph, so 1.3 would imply that either $G$ is a line graph, or it has a decomposition in accordance with 1.3. Proving a result of this kind is our first main goal, but exactly how it goes depends on what we mean by "substantial". To make the theorem as powerful as possible, we need to weaken what we mean by "substantial" as much as we can; but when $L(H)$ gets very small, all sorts of bad things start to happen. One is that the theorem is not true any more. For instance, when $H=K_{3,3}$ or $K_{3,3} \backslash e$ (the graph obtained from $K_{3,3}$ by deleting one edge), then $L(H)$ is not only a line graph but also the complement of a line graph (indeed, it is isomorphic to its own complement). So $L(H)$ can live happily inside bigger graphs that are complements of line graphs, without inducing any kind of decomposition. The best we can hope for, when $L(H)$ is so small, is therefore to prove that either $G$ is a line graph or the complement of a line graph, or has a decomposition of the kind we like. This works for $L\left(K_{3,3}\right)$, but for $L\left(K_{3,3} \backslash e\right)$ the situation is even worse, because this graph is basic in three ways - it is a line graph, the complement of a line graph, and a double split graph. So for Berge graphs $G$ that contain $L\left(K_{3,3} \backslash e\right)$, the best we can hope is that either $G$ is a line graph or the complement of one or a double split graph, or it has a decomposition. And that turns out to be true, but it also explains why the small cases will be something of a headache, as the reader will see.

The best way to partition these cases appears to be as follows. If $H$ is a bipartite subdivision of $K_{4}$, we say that $L(H)$ is degenerate if there is a cycle of $H$ of length four containing the four vertices of $H$ that have degree three in $H$, and nondegenerate otherwise. First we prove the following.

5.1 Let $G$ be Berge, and assume some nondegenerate $L(H)$ is an induced subgraph of $G$, where $H$ is a bipartite subdivision of $K_{4}$. Then either $G$ is a line graph, or $G$ admits a proper 2-join, or $G$ admits a balanced skew partition. In particular, 1.8.1 holds.

Now we consider the case when $G$ contains $L(H)$ for some bipartite subdivision $H$ of a 3-connected $J$, and yet 5.1 does not apply. It turns out that then either $H=K_{3,3}$, or $H$ is a subdivision of $K_{4}$ and $L(H)$ is degenerate. The case when $H=K_{3,3}$ is handled by the next theorem.

5.2 Let $G$ be Berge, and assume it contains $L\left(K_{3,3}\right)$ as an induced subgraph. Then either:

- $G=L\left(K_{3,3}\right)$, or

- for some bipartite subdivision $H$ of $K_{4}, L(H)$ is nondegenerate and is an induced subgraph of one of $G, \bar{G}$, or 
- $G$ admits a balanced skew partition.

In particular, 1.8.2 holds.

The proofs of these two theorems are similar, and we prove them both together. The remaining case, when $H$ is a subdivision of $K_{4}$ and $L(H)$ is degenerate, seems to have a different character, and is best handled by a separate argument later.

The proof of the two theorems above is roughly as follows. We choose a 3-connected graph $J$, as large as possible such that $G$ contains $L(H)$ for some bipartite subdivision $H$ of $J$. (For the the first theorem, we also assume that $L(H)$ includes some nondegenerate $L\left(H^{\prime}\right)$ where $H^{\prime}$ is a bipartite subdivision of $K_{4}$, and for the second theorem, when necessarily $H=K_{3,3}$, we also assume that passing to the complement will not give us a bigger choice of $J$ ). Now we investigate how the remainder of $G$ can attach onto $L(H)$. The edges of $J$ correspond to edge-disjoint paths of $H$, which in turn become vertex-disjoint paths of $L(H)$, which we call "rungs" (we will do the definitions properly later). One thing we find is that the remainder of $G$ can contain alternative rungs paths that could replace one of the rungs in $L(H)$ to give a new $L\left(H^{\prime}\right)$, for some other bipartite subdivision $H^{\prime}$ of the same graph $J$. We find it advantageous to assemble all these alternative rungs in one "strip", for each edge of $J$, and to maximize the union of these strips (being careful that there are no unexpected edges of $G$ between strips). Each strip corresponds to an edge of $J$, and runs between two sets of vertices (called "potatoes" for now) that correspond to vertices of $J$. Let the union of the strips be $Z$ say. Again we ask, how does the remainder of $G$ attach onto this "generalized line graph" $Z$ ? This turns out to be quite pretty. There are only two kinds of vertices in the remainder of $G$, vertices with very few neighbours in $Z$, and vertices with a lot of neighbours. For the first kind, all their neighbours lie either in one of the strips, or in one of the potatoes; and we can show that for any connected set of these "minor" vertices, the union of their neighbours in $Z$ has the same property (they all lie in one strip or in one potato). For the second kind of vertex, they have so many neighbours in $Z$ that all their non-neighbours in any one potato lie inside one strip incident with the potato; and the same is true for the union of the nonneighbours of any anticonnected set of these "major" vertices. In other words, every anticonnected set of these major vertices has a great many common neighbours in $Z$, so many that they separate all the strips from one another, and that is where we find skew partitions. (If there are no major vertices then we need a different argument, but that case is basically easy.)

In this section and the next few, we have to pay for our convention that "path" means "induced path", because here we need paths in the conventional sense, and therefore need to use a different word for them. A track $P$ is a non-null connected graph, not a cycle, in which every vertex has degree $\leq 2$; and its length is the number of edges in it. (Its ends and internal vertices are defined in the natural way.) A track in a graph $H$ means a subgraph of $H$ (not necessarily induced) which is a track. Note that there is a correspondence between the tracks (with at least one edge) in a graph $H$ and the paths in $L(H)$; the edge-set of a track becomes the vertex-set of a path, and vice versa. And two tracks are vertex-disjoint if and only if the corresponding paths are vertex-disjoint and there is no edge of $L(H)$ between them. However, the parity changes; a track in $H$ and the corresponding path in $L(H)$ have lengths differing by one, and therefore of opposite parity.

A branch-vertex of a graph $H$ means a vertex with degree $\geq 3$; and a branch of $H$ means a maximal track $P$ in $H$ such that no internal vertex of $P$ is a branch-vertex. Subdividing an edge $u v$ means deleting the edge $u v$, adding a new vertex $w$, and adding two new edges $u w$ and $w v$. Starting 
with a graph $J$, the effect of repeatedly subdividing edges is to replace each edge of $J$ by a track joining the same pair of vertices, where these tracks are disjoint except for their ends. We call the graph we obtain a subdivision of $J$. Note that $V(J) \subseteq V(H)$. Let $J$ be a 3-connected graph. (We use the convention that a $k$-connected graph must have $>k$ vertices.) If $H$ is a subdivision of $J$ then $V(J)$ is the set of branch-vertices of $H$, and the branches of $H$ are in 1-1 correspondence with the edges of $J$. We say $H$ is cyclically 3-connected if it is a subdivision of some 3-connected graph $J$. (We remind the reader that in this paper, all graphs are simple by definition.)

In general, if $F, K$ are induced subgraphs of $G$ with $V(F \cap K)=\emptyset$, a vertex in $V(K)$ is said to be an attachment of $F$ (or of $V(F)$ ) if it has a neighbour in $V(F)$. We need the following lemma:

5.3 Let $H$ be bipartite and cyclically 3-connected. Then either $H=K_{3,3}$, or $H$ is a subdivision of $K_{4}$, or $H$ has a subgraph $H^{\prime}$ such that $H^{\prime}$ is a subdivision of $K_{4}$ and $L\left(H^{\prime}\right)$ is nondegenerate.

Proof. There is a subgraph of $H$ which is a subdivision of $K_{4}$, and we may assume that it does not satisfy the theorem. Hence there are tracks $p_{1^{-}} \cdots-p_{m}$ (=P say) and $q_{1}-\cdots-q_{n}$ (=Q say) of $H$, vertex-disjoint, such that $p_{1} q_{1}, p_{1} q_{n}, p_{m} q_{1}, p_{m} q_{n}$ are edges, and $m, n \geq 3$ are odd. Suppose every track in $H$ between $\left\{p_{1}, \ldots, p_{m}\right\}$ and $\left\{q_{1}, \ldots, q_{n}\right\}$ uses one of the edges $p_{1} q_{1}, p_{1} q_{n}, p_{m} q_{1}, p_{m} q_{n}$. Then there are no edges between $P$ and $Q$ except the given four, and for every component $F$ of $H \backslash(V(P) \cup V(Q))$, the set of attachments of $F$ in $V(P) \cup V(Q)$ is a subset of one of $V(P), V(Q)$. Since $H$ is cyclically 3 -connected, it follows that $H$ is a subdivision of $K_{4}$ and the theorem holds. So we may assume that there is a track $R$ of $H$, say $r_{1^{-}} \cdots-r_{t}$, from $V(P)$ to $V(Q)$, not using any of $p_{1} q_{1}, p_{1} q_{n}, p_{m} q_{1}, p_{m} q_{n}$. We may assume that $r_{1} \in\left\{p_{1}, \ldots, p_{m-1}\right\}, r_{t} \in\left\{q_{1}, \ldots, q_{n-1}\right\}$, and none of $r_{2}, \ldots, r_{t-1}$ belong to $V(P) \cup V(Q)$. The subgraph $H^{\prime}$ formed by the edges $E(P) \cup E(Q) \cup E(R) \cup\left\{p_{1} q_{n}, p_{m} q_{1}, p_{m} q_{n}\right\}$ (and the vertices of $H$ incident with them) is a subdivision of $K_{4}$, and we may assume it does not satisfy the theorem. There is therefore a cycle of $H^{\prime}$ with vertex set $\left\{r_{1}, r_{t}, p_{m}, q_{n}\right\}$. Since $H$ is bipartite and $p_{m} q_{n}$ is an edge, it follows that $t=2$. Hence not both $r_{1}=p_{1}$ and $r_{2}=q_{1}$, and so $r_{1}=p_{m-1}$ and $r_{2}=q_{n-1}$. By the same argument with $p_{1}, p_{m}$ exchanged, it follows that $r_{1}=p_{2}$, and so $m=3$, and similarly $n=3$. Hence there is a subgraph $J$ of $H$ isomorphic to $K_{3,3}$.

It is helpful now to change the notation. Let $J$ have vertex set $\left\{a_{1}, a_{2}, a_{3}, b_{1}, b_{2}, b_{3}\right\}$, where $a_{1}, a_{2}, a_{3}$ are adjacent to $b_{1}, b_{2}, b_{3}$. Suppose that there is a component $F$ of $H \backslash V(J)$. Since $H$ is cyclically 3-connected, at least two vertices of $J$ are attachments of $F$. If say $a_{1}, b_{1}$ are attachments, choose a track $P$ between $a_{1}, b_{1}$ with interior in $F$; then the union of $P$ and $J \backslash\left\{a_{1} b_{1}, a_{2} b_{2}\right\}$ satisfies the theorem. If say $a_{1}, a_{2}$ are attachments of $F$, choose a track $P$ between $a_{1}, a_{2}$ with interior in $F$; then the union of $P$ and $J \backslash\left\{a_{1} b_{1}, a_{2} b_{3}\right\}$ satisfies the theorem. So we may assume there is no such $F$. Since $H$ is bipartite, it follows that $H=J=K_{3,3}$, and so the theorem holds. This proves 5.3.

If $G, J$ are graphs, we say that $J$ appears in $G$ if there is a bipartite subdivision $H$ of $J$ so that $L(H)$ is isomorphic to an induced subgraph of $G$. We call $L(H)$ an appearance of $J$ in $G$. Note that if $L(H)$ is isomorphic to some induced subgraph $K$ of $G$, there is another subdivision $H^{\prime}$ isomorphic to $H$, made from $H$ by replacing each edge of $H$ by the corresponding vertex of $K$; and now $L\left(H^{\prime}\right)=K$ (rather than just being isomorphic to it). So whenever it is convenient we shall assume that the isomorphism between $L(H)$ and $K$ is just equality, without further explanation. Note in particular that $E(H)=V(K)$, and so some vertices of $G$ are edges of $H$.

When $J=K_{4}$, we have already defined what we mean by a degenerate appearance of $J$. When $J \neq K_{4}$, let us say that an appearance $L(H)$ of $J$ in $G$ is degenerate if $J=H=K_{3,3}$, and otherwise 
it is nondegenerate. So all appearances of any graph $J \neq K_{4}, K_{3,3}$ are nondegenerate. If $J$ is 3 connected, we say a graph $J^{\prime}$ is a $J$-enlargement if $J^{\prime}$ is 3 -connected, and has a proper subgraph which is isomorphic to a subdivision of $J$.

Our goal remains to prove 5.1 and 5.2. Before we start on the main argument, let us observe that it suffices to prove the following.

5.4 Let $G$ be Berge. Let $J$ be a 3-connected graph, such that there is no J-enlargement with a nondegenerate appearance in $G$. Let $L\left(H_{0}\right)$ be an appearance of $J$ in $G$, such that if $L\left(H_{0}\right)$ is degenerate, then $H_{0}=J=K_{3,3}$ and no J-enlargement appears in $\bar{G}$. Then either $G=L\left(H_{0}\right)$, or $H_{0} \neq K_{3,3}$ and $G$ admits a proper 2-join, or $G$ admits a balanced skew partition.

The proof of this will take several sections, but let us see now that 5.4 implies 5.1 and 5.2.

\section{Proof of 5.1, assuming 5.4.}

Let $G$ be Berge, and assume there is a nondegenerate appearance of $K_{4}$ in $G$. Choose a 3connected graph $J$ maximal (under $J$-enlargement) such that there is a nondegenerate appearance of $J$ in $G$; then the hypotheses of 5.4 are satisfied, and the claim follows from 5.4. This proves 5.1.

\section{Proof of 5.2, assuming 5.4.}

Let $G$ be Berge, and let $L\left(H_{0}\right)$ be an appearance of $K_{3,3}$ in $G$, where $H_{0}=K_{3,3}$. We may assume that both $G, \bar{G}$ contain no nondegenerate $L(H)$ where $H$ is a bipartite subdivision of $K_{4}$. By 5.3 , no $K_{3,3}$-enlargement appears in either $G, \bar{G}$. By 5.4 , either $G=L\left(K_{3,3}\right)$, or $G$ admits a balanced skew partition. This proves 5.2.

Now we start on the proof of 5.4. We assume that $L(H)$ is an appearance of $J$ in $G$, and we need to study how the remaining vertices of $G$ attach to $L(H)$. In the remainder of this section we examine how individual vertices attach to $L(H)$, and how connected sets of minor vertices attach. In the next section we think about anticonnected sets of major vertices.

A vertex in $V(G) \backslash V(L(H))$ has a set of neighbours in $V(L(H))$, that we want to investigate; but this set is more conveniently thought of as a subset of $E(H)$, and we begin with some lemmas about subsets of edges of a graph $H$.

5.5 Let $H$ be cyclically 3-connected, and let $C, D$ be subgraphs with $C \cup D=H,|V(C \cap D)| \leq 2$, and $V(C), V(D) \neq V(H)$. Then one of $C, D$ is contained in a branch of $H$.

The proof is clear.

5.6 Let $c_{1}, c_{2}$ be nonadjacent vertices of a graph $H$, such that $H \backslash\left\{c_{1}, c_{2}\right\}$ is connected. For $i=1,2$, let the edges incident with $c_{i}$ be partitioned into two sets $A_{i}, B_{i}$, where $A_{1}, A_{2}$ are both nonempty and at least one of $B_{1}, B_{2}$ is nonempty. Assume that for every edge uv $\in A_{1} \cup A_{2}, H \backslash\{u, v\}$ is connected, and that no vertex of $V(H)$ is incident with all edges in $A_{1} \cup A_{2}$. Then one of the following holds:

1. there is a track in $H$ with first edge in $A_{1}$, second edge in $B_{1}$ (and hence second vertex $c_{1}$ ), last vertex $c_{2}$ and last edge in $A_{2}$, or

2. there is a track in $H$ with first edge in $A_{2}$, second edge in $B_{2}$ (and hence second vertex $c_{2}$ ), last vertex $c_{1}$ and last edge in $A_{1}$. 
Proof. For $i=1,2$ let $X_{i}$ be the set of ends (different from $c_{i}$ ) of edges in $A_{i}$, and define $Y_{i}$ similarly for $B_{i}$. So by hypothesis, $X_{1}, X_{2}$ are nonempty, $\left|X_{1} \cup X_{2}\right| \geq 2$, and we may assume $Y_{1}$ is nonempty. Choose $x_{1} \in X_{1}$ such that $X_{2} \nsubseteq\left\{x_{1}\right\}$ (this is possible since $\left|X_{1} \cup X_{2}\right| \geq 2$ ). Both $Y_{1}$ and $X_{2}$ meet the connected graph $H \backslash\left\{c_{1}, x_{1}\right\}$, and so there is a track in $H \backslash\left\{c_{1}, x_{1}\right\}$ from $Y_{1}$ to $X_{2} \cup Y_{2}$, say $P$, with vertices $p_{1}, \ldots, p_{n}$ say. We may assume that $p_{1} \in Y_{1}$, and no other $p_{i}$ is in $Y_{1}$; and $p_{n} \in X_{2} \cup Y_{2}$, and no other $p_{i}$ is in $X_{2} \cup Y_{2}$. In particular it follows that $c_{2} \notin V(P)$. Since $x_{1} \notin V(P)$ we may assume that $p_{n} \notin X_{2}$ (for otherwise the theorem holds), so $p_{n} \in Y_{2}$. If any vertex of $X_{1}$ is in $P$ then again the theorem holds (since $X_{2}$ is nonempty and none of its vertices are in $P$ ), so we may assume that $P$ is disjoint from $X_{1} \cup X_{2}$. Since $H \backslash\left\{c_{1}, c_{2}\right\}$ is connected, there is a minimal track $Q$ in $H \backslash\left\{c_{1}, c_{2}\right\}$ from $X_{1} \cup X_{2}$ to $V(P)$, and we may assume that only its first vertex ( $q$ say) is in $X_{1} \cup X_{2}$. If $q \in X_{1} \backslash X_{2}$, choose $x \in X_{2}$; if $q \in X_{2} \backslash X_{1}$ choose $x \in X_{1}$; and if $q \in X_{1} \cap X_{2}$ choose $x \in X_{1} \cup X_{2}$ different from $q$. Thus we may assume that $q \in X_{1}$ and there exists $x \in X_{2}$ different from $q$ and hence not in $Q$. So $P \cup Q$ contains a path from $q$ to $B_{2}$ not containing $x$, and hence the theorem holds. This proves 5.6.

If $v$ is a vertex of $H$, the set of edges of $H$ incident with $v$ is denoted by $\delta(v)$ or $\delta_{H}(v)$. Let $H$ be bipartite and cyclically 3 -connected, and let $X$ be some set. We say that $X$ saturates $L(H)$ if for every branch-vertex $v$ of $H$, at most one edge of $\delta_{H}(v)$ is not in $X$ (or equivalently, for every $K_{3}$ subgraph of $L(H)$, at least two of its vertices are in $X$ ). When $H$ is connected and bipartite, we speak of vertices having the same or different biparity depending whether every track between them is even or odd respectively. Two edges of $G$ are disjoint if they have no end in common, and otherwise they meet.

5.7 Let $H$ be bipartite and cyclically 3-connected. Let $X \subseteq E(H)$, such that there is no track in $H$ of even length $\geq 4$, with its end-edges in $X$ and with no other edge in $X$. Then either:

1. $X$ saturates $L(H)$, or

2. there is a branch-vertex $b$ of $H$ with $X \subseteq \delta(b)$, or

3. there is a branch $B$ of $H$ with $X \subseteq E(B)$, or

4. there is a branch $B$ of $H$ with ends $b_{1}, b_{2}$ say, such that $X \backslash E(B)=\delta\left(b_{1}\right) \backslash E(B)$, or

5. there is a branch $B$ of $H$ of odd length with ends $b_{1}, b_{2}$ say, such that

$$
X \backslash E(B)=\left(\delta\left(b_{1}\right) \cup \delta\left(b_{2}\right)\right) \backslash E(B),
$$

or

6. there are two vertices $c_{1}, c_{2}$ of $H$, of different biparity and not in the same branch of $H$, such that $X=\delta\left(c_{1}\right) \cup \delta\left(c_{2}\right)$.

In particular, either statements 1 or 6 hold, or there are at most two branch-vertices of $H$ incident with more than one edge in $X$; and exactly two only if statement 5 holds.

Proof. The second assertion (the final sentence) follows from the first, because if statements 2,3 or 4 hold then there is at most one branch-vertex incident with more than one edge in $X$; while if 
$B, b_{1}, b_{2}$ are as in statement 5 , then since $B$ is odd, it follows that $b_{1}, b_{2}$ have no common neighbour, and so no branch-vertex different from $b_{1}, b_{2}$ is incident with more than one edge in $X$. So it remains to prove the first assertion.

(1) We may assume that there are two disjoint edges in $X$.

For if not, then by König's theorem, there is a vertex of $H$ incident with every edge in $X$, and then one of statements 2,3 of the theorem hold. This proves (1).

(2) If there is a branch $B$ of $H$ such that every edge in $X$ has at least one end in $V(B)$ then the theorem holds.

For suppose there is a such a branch $B$, and let $C \subseteq B$ be a track, minimal such that every edge in $X$ has an end in $V(C)$. By (1) we may assume that $C$ has length $\geq 1$. Let $c_{1}, c_{2}$ be the ends of $C$. For $i=1,2$ let $A_{i}$ be the set of edges in $\delta\left(c_{i}\right)$ that are in $X$ and not in $C$; and let $B_{i}$ be the set of edges in $\delta\left(c_{i}\right)$ that are not in $X$ and not in $C$. From the minimality of $C$, it follows that $A_{1}, A_{2}$ are both nonempty.

Suppose first that $c_{1}, c_{2}$ have the same biparity. Choose $c_{i} a_{i} \in A_{i}$ for $i=1,2$, if possible such that $a_{1} \neq a_{2}$. Since $c_{1}, c_{2}$ belong to the same branch of $H$ and $H$ is cyclically 3 -connected, it follows that there is a track in $H \backslash\left\{c_{1}, c_{2}\right\}$ from $a_{1}$ to $a_{2}$; and therefore there is a track $T$ in $H$ from $c_{1}$ to $c_{2}$ with end-edges $c_{1} a_{1}$ and $c_{2} a_{2}$. Since $c_{1}, c_{2}$ have the same biparity, it follows that $T$ is even; and since only its end-edges are in $X$ (because every edge in $X$ either belongs to $C$ or is incident with one of $\left.c_{1}, c_{2}\right)$, it follows from the hypothesis of the theorem that $T$ has length 2 , that is, $a_{1}=a_{2}$. We deduce that there is a vertex $a \in V(H)$ such that $A_{i}=\left\{c_{i} a\right\}$ for $i=1,2$. Now there is only one branch of $H$ containing $c_{1}$ and $c_{2}$, since $J$ is simple, so $a$ is not in the interior of a branch, and therefore it is a branch-vertex. Moreover it does not belong to the branch $B$, for the same reason, and so $C=B$ and $c_{1}, c_{2}$ are branch-vertices. Choose a branch-vertex $b$ of $H$ different from $c_{1}, c_{2}, a$, and choose three paths $P_{1}, P_{2}, P_{3}$ between $b$ and $c_{1}, c_{2}, a$ respectively, pairwise disjoint except for $b$. So $P_{1}$ and $P_{2}$ have lengths of the same parity, and $P_{3}$ has length of different parity. By (1) we may assume there is an edge in $X$ not incident with $a$, and any such edge belongs to $C$; so for $i=1,2$ there is a minimal subtrack $Q_{i}$ of $C$ containing $c_{i}$ and an edge in $X$. If $Q_{1}=C$ then (since $C$ has even length) $P_{1} \cup P_{2}$ is the interior of an even track with end-edges in $X$ and no internal edges in $X$, contrary to the hypothesis. So $c_{2}$ is not a vertex of $Q_{1}$, and similarly $c_{1}$ is not in $Q_{2}$. From the track $Q_{1}-c_{1}-P_{1}-b-P_{2}-c_{2}-a$ and the hypothesis it follows that $Q_{1}$ is even; and from the track $Q_{1}-c_{1}-P_{1}-b-P_{3}-a-c_{2}$ and the hypothesis it follows that $Q_{1}$ is odd, a contradiction.

We may assume therefore that $c_{1}, c_{2}$ have different biparity. It follows that no vertex of $V(H)$ is incident with all edges in $A_{1} \cup A_{2}$. Let $H^{\prime}$ be the graph obtained from $H$ by deleting the internal vertices and edges of $C$. There is no track $T$ in $H^{\prime}$ with first edge in $A_{1}$, second edge in $B_{1}$ (and hence second vertex $c_{1}$ ), last vertex $c_{2}$ and last edge in $A_{2}$; for any such track would be even, since $c_{1}, c_{2}$ have opposite biparity, and have length $\geq 4$, and have only its end-edges in $X$, contrary to the hypothesis. A similar statement holds with $c_{1}, c_{2}$ exchanged. By 5.6 applied to $H^{\prime}$, it follows that $B_{1} \cup B_{2}=\emptyset$, and so one of statements $3,4,5$ of the theorem holds. This proves (2).

(3) There do not exist three tracks of $H$ with an end (b say) in common and otherwise vertex- 
disjoint, such that each contains an edge in $X$, and at least two of the three edges of the tracks incident with $b$ do not belong to $X$.

For suppose that $P_{1}, P_{2}, P_{3}$ are three such tracks, where $P_{i}$ is between $a_{i}$ and $b$, for $1 \leq i \leq 3$. We may assume that for each $i$, the only edge of $P_{i}$ in $X$ is the edge incident with $a_{i}$. Now two of $P_{1}, P_{2}, P_{3}$ have lengths of the same parity, say $P_{1}, P_{2}$; and their union is an even track with end-edges in $X$ and its other edges not in $X$. By hypothesis it has length 2 , and so $P_{1}, P_{2}$ both have length 1 . But then at most one edge of $P_{1} \cup P_{2} \cup P_{3}$ incident with $b$ does not belong to $X$, a contradiction. This proves (3).

(4) There do not exist a connected subgraph $A$ of $H \backslash X$ and three mutually disjoint edges $x_{1}, x_{2}, x_{3} \in X$ such that each $x_{i}$ has at least one end in $V(A)$.

For suppose such $A, x_{1}, x_{2}, x_{3}$ exist. We may assume $A$ is a maximal connected subgraph of $H \backslash X$. For $1 \leq i \leq 3$ let $x_{i}$ have ends $a_{i}, b_{i}$, where $a_{1}, a_{2}, a_{3}$ have the same biparity. Let $K$ be the graph with vertex set $\left\{a_{1}, a_{2}, a_{3}, b_{1}, b_{2}, b_{3}\right\}$, in which two vertices of $K$ are adjacent if there is a track in $A$ joining them not using any other vertex of $K$. Since $A$ is connected and meets all of $x_{1}, x_{2}, x_{3}$ it follows that there is a component of $K$ containing an end of each of these three edges. If some two of $a_{1}, a_{2}, a_{3}$ are adjacent in $K$, then the corresponding track in $A$ is even, contrary to the hypothesis of the theorem; so $a_{1}, a_{2}, a_{3}$ are pairwise nonadjacent in $K$, and similarly so are $b_{1}, b_{2}, b_{3}$, and therefore all the edges of $K$ join some $a_{i}$ to some $b_{j}$. Also, by (3) it follows that $a_{3}$ is not adjacent in $K$ to both $b_{1}$ and $b_{2}$, and five similar statements. Since there is a component of $K$ containing an end of each of $x_{1}, x_{2}, x_{3}$, we may assume that $a_{1} b_{3}, b_{2} a_{3}, a_{3} b_{3} \in E(K)$, and the only other possible edges of $K$ are $a_{1} b_{1}, a_{2} b_{2}, a_{2} b_{1}$. In particular, there are no more edges of $K$ incident with $a_{3}$ or $b_{3}$. Let the tracks in $A$ corresponding to $a_{1} b_{3}, b_{2} a_{3}, a_{3} b_{3} \in E(K)$ be $P_{1}, P_{2}, P_{3}$ respectively. Since $P_{3}$ joins the adjacent vertices $a_{3}, b_{3}$ and does not use the edge $x_{3}$, it follows that $P_{3}$ has nonempty interior. Choose a maximal connected subgraph $S$ of $A$ including the interior of $P_{3}$ and not containing either of $a_{3}, b_{3}$. Since there are no more edges of $K$ incident with $a_{3}$ or $b_{3}$, it follows that none of $a_{1}, b_{1}, a_{2}, b_{2}$ is in $V(S)$, and therefore $S$ is vertex-disjoint from $P_{1}$ and $P_{2}$ as well. Consequently the only edges of $A$ between $V(S) \cup\left\{a_{3}, b_{3}\right\}$ and the remainder of $H$ are incident with $a_{3}$ or $b_{3}$. Since $H$ is cyclically 3 -connected and $a_{3}, b_{3}$ are adjacent, it follows that $H \backslash\left\{a_{3}, b_{3}\right\}$ is connected, and therefore there is an edge $s v$ of $H$ such that $s \in V(S)$ and $v \in V(H) \backslash\left(V(S) \cup\left\{a_{3}, b_{3}\right\}\right)$. Since $S$ is maximal it follows that $s v \notin E(A)$; and since $A$ is maximal, it follows that $s v \in X$; and from the symmetry we may assume $v \notin\left\{a_{1}, b_{1}\right\}$. Choose a minimal track in $S$ between $s$ and the interior of $P_{3}$; then it can be extended via a subpath of $P_{3}$ and via $s v$ to become a track $P_{4}$ in $H$, of length $\geq 2$, from $v$ to $b_{3}$, using none of $a_{1}, b_{1}, a_{3}$, and with only its first edge in $X$. But then the tracks $b_{1}-a_{1}-P_{1}-b_{3}, P_{4}$, and the one-edge track made by $x_{3}$, violate (3). This proves (4).

We may assume that statement 1 of the theorem does not hold, and so there is a branch-vertex of $H$ incident with $\geq 2$ edges not in $X$. Hence there is a connected subgraph $A$ of $H \backslash X$, containing a branch-vertex and at least two edges incident with it. Choose such a subgraph $A$ maximal. It follows that $V(A)$ is not contained in any branch of $H$. By (4), there is no 3-edge matching among the edges in $X$ that meet $V(A)$; and since this set of edges forms a bipartite subgraph, it follows from König's theorem that there are two vertices $c_{1}, c_{2} \in V(G)$ such that every edge in $X$ with an end in $V(A)$ is incident with one of $c_{1}, c_{2}$. From the maximality of $A$, every edge of $H$ between $V(A)$ 
and $V(H) \backslash V(A)$ belongs to $X$ and therefore is incident with one of $c_{1}, c_{2}$; and so there are two subgraphs $C, D$ of $H$ with $V(C)=V(A) \cup\left\{c_{1}, c_{2}\right\}, V(D)=(V(H) \backslash V(A)) \cup\left\{c_{1}, c_{2}\right\}$ and $C \cup D=H$.

Now $V(C)$ is not contained in a branch of $H$, because it contains $V(A)$ and we already saw that $V(A)$ is not contained in a branch; and we may assume that $V(D)$ is not contained in a branch by (2), since every edge in $X$ has an end in $V(D)$. But $V(D) \neq V(G)$ since $|V(C)| \geq|V(A)| \geq 3>$ $|V(C \cap D)|$; and since $H$ is cyclically 3-connected, it follows that $V(C)=V(G)$. Hence every edge in $X$ is incident with one of $c_{1}, c_{2}$. For $i=1,2$ let $A_{i}=\delta\left(c_{i}\right) \cap X$, and let $B_{i}=\delta\left(c_{i}\right) \backslash A_{i}$. By (2), we may assume that $c_{1}, c_{2}$ do not belong to the same branch. Consequently $c_{1}, c_{2}$ are nonadjacent, and $H \backslash\left\{c_{1}, c_{2}\right\}$ is connected, by 5.5. By (1) we may assume that there exist disjoint edges $a_{1} c_{1} \in A_{1}$ and $a_{2} c_{2} \in A_{2}$. Take a minimal track in $H \backslash\left\{c_{1}, c_{2}\right\}$ between $a_{1}, a_{2}$; then by the hypothesis of the theorem, this track has odd length, and so $c_{1}, c_{2}$ have opposite biparity. There is therefore no track $T$ in $H$ with first edge in $A_{1}$, second edge in $B_{1}$ (and hence second vertex $c_{1}$ ), last vertex $c_{2}$ and last edge in $A_{2}$; and a similar statement holds with $c_{1}, c_{2}$ exchanged. By 5.6, it follows that $B_{1}, B_{2}=\emptyset$, and therefore statement 6 of the theorem holds. This proves 5.7.

Suppose that $L(H)$ is an appearance of $J$ in $G$. We recall that $H$ is a subdivision of $J$, and $L(H)$ is an induced subgraph of $G$. If $X \subseteq V\left(L(H)\right.$, we say that $X$ is local if either $X \subseteq \delta_{H}(v)$ for some $v \in V(J)$, or $X$ is a subset of the edge-set of some branch of $H$. We say a vertex $v \in V(G) \backslash V(L(H))$ is major (with respect to $L(H)$ ) if the set of its neighbours in $L(H)$ saturates $L(H)$.

5.8 Let $G$ be Berge, let $J$ be a 3-connected graph, and let $L(H)$ be an appearance of $J$ in $G$. For each vertex $v$ of $J$, let $N_{v}$ be the set of edges of $H$ incident with $v$; and for each edge uv of $J$, let $R_{u v}$ be the path of $L(H)$ with vertex set the set of edges of the branch of $H$ between $u$ and $v$. Let $F \subseteq V(G) \backslash V(L(H))$ be connected, such that the set of attachments of $F$ in $L(H)$ is not local. Assume that no member of $F$ is major. Then there is a path $P$ of $G$ with $V(P) \subseteq F$ and with ends $p_{1}$ and $p_{2}$, such that either:

1. there are vertices $c_{1}, c_{2}$ of $H$, not in the same branch of $H$, such that for $i=1,2 p_{i}$ is complete in $G$ to $N_{c_{i}}$, and there are no other edges between $V(P)$ and $V(L(H))$, or

2. there is an edge $b_{1} b_{2}$ of $J$ such that one of the following holds (for $i=1,2, r_{i}$ denotes the unique vertex in $N_{b_{i}} \cap V\left(R_{b_{1} b_{2}}\right)$ ):

(a) $p_{1}$ is adjacent in $G$ to all vertices in $N_{b_{1}} \backslash\left\{r_{1}\right\}$, and $p_{2}$ has a neighbour in $R_{b_{1} b_{2}} \backslash r_{1}$, and every edge from $V(P)$ to $V(L(H)) \backslash\left\{r_{1}\right\}$ is either from $p_{1}$ to $N_{b_{1}} \backslash\left\{r_{1}\right\}$, or from $p_{2}$ to $V\left(R_{b_{1} b_{2}}\right) \backslash\left\{r_{1}\right\}$, or

(b) for $i=1,2, p_{i}$ is adjacent in $G$ to all vertices in $N_{b_{i}} \backslash\left\{r_{i}\right\}$, and there are no other edges between $V(P)$ and $V(L(H))$ except possibly $p_{1} r_{1}, p_{2} r_{2}$, and $P$ has the same parity as $R_{b_{1} b_{2}}$, or

(c) $p_{1}=p_{2}$, and $p_{1}$ is adjacent to all vertices in $\left(N_{b_{1}} \cup N_{b_{2}}\right) \backslash\left\{r_{1}, r_{2}\right\}$, and all neighbours of $p_{1}$ in $V(L(H))$ belong to $N_{b_{1}} \cup N_{b_{2}} \cup V\left(R_{b_{1} b_{2}}\right)$, and $R_{b_{1} b_{2}}$ is even, or

(d) $r_{1}=r_{2}$, and for $i=1,2, p_{i}$ is adjacent in $G$ to all vertices in $N_{b_{i}} \backslash\left\{r_{i}\right\}$, and there are no other edges between $V(P)$ and $V(L(H)) \backslash\left\{r_{1}\right\}$, and $P$ is even.

Proof. We remark that the set $N_{v}$ is just the set $\delta_{H}(v)$, but now we are going to think of it as a subset of the vertex set of $L(H)$ and it is convenient to change notation. We may assume $F$ is 
minimal such that its set of attachments is not local. Let $X$ be the set of attachments of $F$ in $L(H)$. Suppose first that $|F|=1, F=\{y\}$ say. Apply 5.7 to $H, X$. Now 5.7.1 is false since by hypothesis $y$ is not major, and 5.7.2 and 5.7.3 are false since $X$ is not local. So one of 5.7.4-6 holds, and the claim follows. Consequently we may assume that $|F| \geq 2$.

(1) There exist two attachments $x_{1}, x_{2}$ of $F$ such that $\left\{x_{1}, x_{2}\right\}$ is not local.

For $X \subseteq E(H)$. If there exists $x_{1} \in X$ not incident in $H$ with a branch-vertex, and in some branch $B$, choose any $x_{2} \in X$ not in $B$; then $\left\{x_{1}, x_{2}\right\}$ is not local. So we may assume that every edge in $X$ is incident with a branch-vertex of $H$. Choose $x_{1} \in X$, in some branch $B_{1}$ of $H$, and incident with a branch-vertex $b_{1}$. There exists $x_{2} \in X$ not incident with $b_{1}$, and we may assume that $x_{2} \in E\left(B_{1}\right)$, for otherwise $\left\{x_{1}, x_{2}\right\}$ is not local. Hence $x_{2}$ is incident with the other end $b_{2}$ say of $B_{1}$. There exists $x_{3} \in X$ not belonging to $E(B)$, and it cannot share an end both with $x_{1}$ and with $x_{2}$, so we may assume $x_{3}$ is not incident with $b_{1}$. But then $\left\{x_{1}, x_{3}\right\}$ is not local, as required. This proves (1).

From the minimality of $F$, it follows that $F$ is minimal such that $x_{1}$ and $x_{2}$ are both attachments of $F$, and so (since $x_{1}$ and $x_{2}$ are nonadjacent), $F$ is the interior of a path with vertices $x_{1}, p_{1}, \ldots, p_{n}, x_{2}$ in order. Let $X_{1}$ be the set of attachments in $L(H)$ of $F \backslash\left\{p_{n}\right\}$, and let $X_{2}$ be the attachments of $F \backslash\left\{p_{1}\right\}$. From the minimality of $F, X_{1}$ and $X_{2}$ are both local.

(2) If there is an edge uv of $J$ such that $X_{1} \subseteq N_{u}$ and $X_{2} \subseteq V\left(R_{u v}\right)$ then the theorem holds.

For let the ends of $R_{u v}$ be $r_{1}, r_{2}$ where $r_{1} \in N_{u}$. Since $X$ is not local, it follows that $p_{1}$ has a neighbour in $N_{u} \backslash\left\{r_{1}\right\}$ and $p_{n}$ has a neighbour in $V\left(R_{u v}\right) \backslash\left\{r_{1}\right\}$. If $p_{1}$ is adjacent to every vertex in $N_{u} \backslash\left\{r_{1}\right\}$ then statement 2.a of the theorem holds, so we may assume $p_{1}$ has a neighbour $s_{1}$ and a nonneighbour $s_{2}$ in $N_{u} \backslash\left\{r_{1}\right\}$. Let $Q$ be the path between $r_{2}$ and $s_{1}$ with interior in $F \cup V\left(R_{u v} \backslash\left\{r_{1}\right\}\right)$. Choose $w \in V(J)$ such that $s_{1} \in V\left(R_{u w}\right)$. Now $H$ is a subdivision of a 3 -connected graph, so if we delete all edges of $H$ incident with $u$ except $s_{1}$, the graph we produce is still connected. Consequently there is a track of $H$ from $u$ to $v$ with first edge $s_{1}$; and hence there is a path $S_{1}$ of $L(H)$ from $s_{1}$ to $r_{2}$, vertex-disjoint from $V\left(R_{u v}\right) \cup N_{u}$ except for its ends. Indeed, if we delete from $H$ both the vertex $w$ and all edges incident with $u$ except $s_{2}$, the graph remains connected; so there is a path $S_{2}$ of $L(H)$ between $s_{2}$ and $r_{2}$, vertex-disjoint from $R_{u v} \cup N_{u} \cup V\left(R_{u w}\right) \cup N_{w}$ except for its ends. Now $S_{1}$ and $S_{2}$ have the same parity since $H$ is bipartite. Yet $S_{1}$ can be completed via $r_{2}-Q-s_{1}$ and $S_{2}$ can be completed via $r_{2}-Q-s_{1}-s_{2}$, a contradiction. This proves (2).

(3) If there are nonadjacent vertices $v_{1}, v_{2} \in V(J)$ such that $X_{i} \subseteq N_{v_{i}}$ for $i=1,2$, then the theorem holds.

Let $A_{1}$ be the set of vertices in $N_{v_{1}}$ adjacent to $p_{1}$, and $B_{1}=N_{v_{1}} \backslash A_{1}$; and let $A_{2}$ be the set of vertices in $N_{v_{2}}$ adjacent to $p_{n}$, and $B_{2}=N_{v_{2}} \backslash A_{2}$. So $X=A_{1} \cup A_{2}$. If both $B_{1}$ and $B_{2}$ are empty then statement 1 of the theorem holds, so we may assume that at least one of $B_{1}, B_{2}$ is nonempty. Certainly $A_{1}$ and $A_{2}$ are both nonempty, so there is a track in $H$ from $v_{1}$ to $v_{2}$ with end-edges in $A_{1}$ and $A_{2}$ respectively. Hence there is a path $S_{1}$ in $L(H)$ from $A_{1}$ to $A_{2}$, vertex-disjoint from $N_{v_{1}} \cup N_{v_{2}}$ except for its ends. Since $X=A_{1} \cup A_{2}$ is not local, there is no $w \in V(J)$ with $A_{1} \cup A_{2} \subseteq N_{w}$. 
Hence we can apply 5.6, and we deduce (possibly after exchanging $v_{1}$ and $v_{2}$ ) that there is a path $S_{2}$ in $L(H)$ with first vertex in $A_{1}$, second vertex in $B_{1}$, last vertex in $A_{2}$, and otherwise disjoint from $N_{v_{1}} \cup N_{v_{2}}$. Since $H$ is bipartite, $S_{1}$ and $S_{2}$ have opposite parity; but they can both be completed via $F$, a contradiction. This proves (3).

(4) If there are adjacent vertices $v_{1}, v_{2} \in V(J)$ such that $X_{i} \subseteq N_{v_{i}}$ for $i=1,2$, then the theorem holds.

For $i=1,2$ let $r_{i}$ be the end of $R_{v_{1} v_{2}}$ in $N_{v_{i}}$. Let $A_{1}$ be the set of vertices in $N_{v_{1}} \backslash\left\{r_{1}\right\}$ adjacent to $p_{1}$, and $B_{1}=N_{v_{1}} \backslash\left(A_{1} \cup r_{1}\right)$; and define $A_{2}, B_{2}$ similarly. Then $X \subseteq A_{1} \cup A_{2} \cup\left\{r_{1}, r_{2}\right\}$. By (2) we may assume that $A_{1}$ and $A_{2}$ are both nonempty. Suppose that both $B_{1}$ and $B_{2}$ are empty. There is a cycle in $J$ of length $\geq 4$ using the edge $v_{1} v_{2}$, and so there is a path in $L(H)$ of length $\geq 2$ from $A_{1}$ to $A_{2}$ with no internal vertex in $N_{v_{1}} \cup V\left(R_{v_{1} v_{2}}\right) \cup N_{v_{2}}$. The union of this path with $R_{v_{1} v_{2}}$ induces a hole, and so does its union with $F$, and therefore these two paths have lengths of the same parity. Consequently either statement 2.b or 2.d of the theorem holds. So we may assume that at least one of $B_{1}, B_{2}$ is nonempty. There is a path $S_{1}$ from $A_{1}$ to $A_{2}$ with no vertex in $N_{v_{1}} \cup N_{v_{2}} \cup V\left(R_{v_{1} v_{2}}\right)$ except for its ends. Suppose that there is no vertex $w \in V(J)$ with $A_{1} \cup A_{2} \subseteq N_{w}$. Then we can apply 5.6 to the graph obtained from $H$ by deleting the edges and internal vertices of the branch between $v_{1}$ and $v_{2}$. We deduce (possibly after exchanging $v_{1}$ and $v_{2}$ ) that there is a path $S_{2}$ of $L(H)$ with first vertex in $A_{1}$, second vertex in $B_{1}$, last vertex in $A_{2}$, and otherwise disjoint from $N_{v_{1}} \cup N_{v_{2}} \cup V\left(R_{v_{1} v_{2}}\right)$. Since $H$ is bipartite, $S_{1}$ and $S_{2}$ have opposite parity; but they can both be completed via $F$, a contradiction. Consequently there is a vertex $w \in V(J)$ with $A_{1} \cup A_{2} \subseteq N_{w}$. Since $H$ is bipartite, and there is a 2-edge track of $H$ between $v_{1}, v_{2}$ (via $w$ ), it follows that the branch of $H$ with ends $v_{1}, v_{2}$ has even length, and therefore $R_{v_{1} v_{2}}$ has odd length, and in particular $r_{1} \neq r_{2}$. Since $\left|N_{v_{i}} \cap N_{w}\right| \leq 1$ (since $J$ is simple) it follows that $\left|A_{i}\right|=1, A_{i}=\left\{a_{i}\right\}$ say, for $i=1,2$. Since $X$ is not local it is not a subset of $N_{w}$ and so there is a vertex of $R_{v_{1} v_{2}}$ in $X$. Since $X_{i} \subseteq N_{v_{i}}$ for $i=1,2$, no internal vertex of $R_{v_{1} v_{2}}$ is in $X$, so we may assume that $r_{1} \in X$. Since $r_{1} \notin N_{v_{2}}$ it follows that $r_{1} \notin X_{2}$, and hence $p_{1}$ is the only vertex in $F$ adjacent to $r_{1}$. Now the hole $p_{1}-\cdots-p_{n}-a_{2}-a_{1}-p_{1}$ is even, and so $n$ is even. If we delete the vertex $v_{2}$ and the edge $a_{1}$ from $H$, what remains is still connected, and so contains a track from $w$ to $v_{1}$. Hence there is a path $T$ in $L(H)$ from some $a_{3} \in N(w)$ to $r_{1}$, disjoint from $N_{v_{2}} \cup a_{1}$. But $T$ can be completed to a hole via $r_{1}-R_{v_{1} v_{2}}-r_{2}-a_{2}-a_{3}$ and via $r_{1}-p_{1}-\cdots-p_{n}-a_{2}-a_{3}$, and these two completions have different parity, a contradiction. This proves (4).

(5) If $X_{1} \cap X_{2}$ is nonempty, and in particular if one of $p_{2}, \ldots, p_{n-1}$ has a neighbour in $L(H)$, then the theorem holds.

For any neighbour in $L(H)$ of one of $p_{2}, \ldots, p_{n-1}$ belongs to $X_{1} \cap X_{2}$, so assume $x \in X_{1} \cap X_{2}$. Then $x \in V\left(R_{v_{1} v_{2}}\right)$ for a unique edge $v_{1} v_{2}$ of $J$, and $x \in N_{v}$ for at most two $v \in V(J)$, namely $v_{1}$ and $v_{2}$. Since both $X_{1}$ and $X_{2}$ are local, each is a subset of one of $N_{v_{1}}, N_{v_{2}}, V\left(R_{v_{1} v_{2}}\right)$, and they are not both subsets of the same one. So we may assume that $X_{1} \subseteq N_{v_{1}}$. Hence either $X_{2} \subseteq N_{v_{2}}$ or $X_{2} \subseteq V\left(R_{v_{1} v_{2}}\right)$, and therefore the theorem holds by (5) or (2). This proves (5).

(6) If there is a vertex $u$ and an edge $v_{1} v_{2}$ of $J$ such that $X_{1} \subseteq N_{u}$ and $X_{2} \subseteq V\left(R_{v_{1} v_{2}}\right)$ then the theorem holds. 
For by (2) we may assume $u$ is different from $v_{1}$ and $v_{2}$. Choose a cycle $C_{1}$ of $H$ using the branch between $v_{1}$ and $v_{2}$ and not using $u$, and choose a minimal track $S$ in $H \backslash\left\{v_{1}, v_{2}\right\}$ between $u$ and $V\left(C_{1}\right)$. Let the ends of $S$ be $u$ and $w$ say. Hence in $L(H)$ there are three vertex-disjoint paths, from $N_{v_{1}}, N_{v_{2}}, N_{u}$ respectively to $N_{w}$, and there are no edges between them except in the triangle $T$ formed by their ends in $N_{w}$. If $p_{n}$ has a unique neighbour (say $r$ ) in $R_{v_{1} v_{2}}$, then $r$ can be linked onto the triangle $T$, contrary to 2.4. If $p_{n}$ has two nonadjacent neighbours in $R_{v_{1} v_{2}}$, then $p_{n}$ can be linked onto the triangle $T$, contrary to 2.4 . So $p_{n}$ has exactly two neighbours in $R_{v_{1} v_{2}}$, and they are adjacent. If $p_{1}$ is adjacent to all of $N_{u}$, then statement 1 of the theorem holds, so we may assume that $p_{1}$ has a neighbour and a non-neighbour in $N_{u}$. Let $A$ be the neighbours of $p_{1}$ in $N_{u}$ and $B=N_{u} \backslash A$. In $H$ there is a cycle $C_{2}$ using the branch between $v_{1}$ and $v_{2}$, and using an edge in $A$ and an edge in $B$. (To see this, divide $u$ into two adjacent vertices, one incident with the edges in $A$ and the other with those in $B$, and use Menger's theorem to deduce that there are two vertex-disjoint paths between these two vertices and $\left\{v_{1}, v_{2}\right\}$.) Hence in $G$, there is a path between $N_{v_{1}}$ and $N_{v_{2}}$ using a unique edge of $N(u)$, and that edge is between a vertex $a \in A$ say and some vertex in $B$. Hence $a$ can be linked onto the triangle formed by $p_{n}$ and its two neighbours in $R_{v_{1} v_{2}}$, a contradiction. This proves (6).

(7) If there are edges $u_{1} v_{1}$ and $u_{2} v_{2}$ of $J$ with $X_{i} \subseteq V\left(R_{u_{i} v_{i}}\right)$ for $i=1,2$, then the theorem holds.

For in this case it follows that the edges $u_{1} v_{1}$ and $u_{2} v_{2}$ are different, and hence we may assume that $v_{2}$ is different from $u_{1}$ and $v_{1}$, and $v_{1}$ is different from $u_{2}$ and $v_{2}$; possibly $u_{1}=u_{2}$. If $p_{1}$ has exactly two neighbours in $R_{u_{1} v_{1}}$ and they are adjacent, and also $p_{n}$ has exactly two neighbours in $R_{u_{2} v_{2}}$ and they are adjacent, then statement 1 of the theorem holds; so we may assume that $p_{1}$ has either only one neighbour, or two nonadjacent neighbours, in $R_{u_{1} v_{1}}$. There is a cycle in $H$ using the branch between $u_{1}$ and $v_{1}$, and using $u_{2}$ and not $v_{2}$ (since $J \backslash v_{2}$ is 2-connected). There correspond two paths in $L(H)$, say $P$ and $Q$, from $N_{u_{1}}$ and $N_{v_{1}}$ respectively to $N_{u_{2}}$, disjoint from each other, and there is a third path $R$ say from $p_{1}$ to $N_{u_{2}}$ via $F$ and a subpath of $R_{u_{2} v_{2}}$. There are no edges between these paths except within the triangle $T$ formed by their ends in $N_{u_{2}}$. If $p_{1}$ has only one neighbour $r \in R_{u_{1} v_{1}}$, then we may assume that $r$ is in the interior of $R_{u_{1} v_{1}}$, by (6), and so $r$ can be linked onto $T$, contrary to 2.4. If $p_{1}$ has two nonadjacent neighbours in $R_{u_{1} v_{1}}$, then $p_{1}$ can be linked onto $T$, again a contradiction. This proves (7).

But (2)-(7) cover all the possibilities for the local sets $X_{1}$ and $X_{2}$, and so this proves 5.8 .

\section{Major attachments to a line graph}

We continue to study appearances $L(H)$ of a 3-connected graph $J$ in a Berge graph $G$. In this section we study anticonnected sets of major vertices, and their common neighbours in $L(H)$.

An appearance $L(H)$ of $J$ in $G$ is overshadowed if there is a branch $B$ of $H$ with odd length $\geq 3$, with ends $b_{1}, b_{2}$, such that some vertex of $G$ is nonadjacent in $G$ to at most one vertex in $\delta_{H}\left(b_{1}\right)$ and at most one in $\delta_{H}\left(b_{2}\right)$. Thus for instance an appearance is overshadowed if there is a major vertex and some branch has odd length at least 3. This section is devoted to proving the following. 
6.1 Let $G$ be Berge, let $L(H)$ be an appearance in $G$ of a 3-connected graph $J$, and let $Y$ be an anticonnected set of major vertices. Assume that the set of all $Y$-complete vertices in $L(H)$ does not saturate $L(H)$. Then either

- $J=K_{3,3}$ or $K_{4}$, and there is an overshadowed appearance of $J$ in $G$, or

- $J=K_{3,3}$ or $K_{4}, L(H)$ is degenerate, and there is an overshadowed appearance of $J$ in $\bar{G}$, or

- $J=K_{3,3}, L(H)$ is degenerate, and there is a J-enlargement that appears in $\bar{G}$, or

- $J=K_{4}$ and $|V(H)|=6$, or

- $J=K_{4}$ and $L(H)$ is degenerate, and there exist nonadjacent $y, y^{\prime} \in Y$ with the following property. Let the 4-cycle in $H$ formed by the branch-vertices of $H$ have edges a-b-c-d in order. Let $p$ be the third edge of $H$ such that $a, b, p$ have a common end, and similarly let $b, c, q$ have a common end, and $c, d, r$ and $d, a, s$. Then (up to symmetry) the neighbours of $y$ in $L(H)$ are $a, b, d, q, r$ and possibly $c$, and the neighbours of $y^{\prime}$ in $L(H)$ are $b, c, d, p, s$ and possibly $a$.

Proof. We may assume that $Y$ is minimal such that it is anticonnected and its common neighbours do not saturate $L(H)$. Let $X$ be the set of all $Y$-complete vertices in $L(H)$. Choose two vertices of $L(H)$, both incident in $H$ with the same branch-vertex of $H$, and both not in $X$. Then there is an antipath joining them with interior in $Y$, and the common neighbours of the interior of this antipath do not saturate $L(H)$. From the minimality of $Y$ it follows that this antipath contains all vertices in $Y$. Consequently, $Y$ is the vertex set of an antipath with ends $y_{1}, y_{2}$, say. From the hypothesis, $|Y| \geq 2$, since the neighbours of any vertex in $Y$ saturate $L(H)$, so $y_{1}, y_{2}$ are distinct. Now for $i=1,2, Y \backslash\left\{y_{i}\right\}$ is anticonnected; let $X_{i}$ be the set of $Y \backslash\left\{y_{i}\right\}$-complete vertices in $L(H)$ that are not in $X$. So $X \cup X_{i}$ is the set of all $Y \backslash\left\{y_{i}\right\}$-complete vertices in $L(H)$. From the minimality of $Y$, both $X \cup X_{1}$ and $X \cup X_{2}$ saturate $L(H)$. In terms of $H$, we see that $X, X_{1}, X_{2}$ are mutually disjoint subsets of $E(H)$, and for every branch-vertex $b$ of $H$ and for $i=1,2$, at most one edge of $H$ incident with $b$ does not belong to $X \cup X_{i}$.

(1) If the branch-vertices of $H$ form a 4-cycle $C$ and $X$ consists of at most three edges of $C$, then the theorem holds.

For in this case $H$ has only four branch-vertices and $J=K_{4}$. Let the edges of $C$ be $a, b, c, d$ in order, and let $p, q, r, s$ be edges of $H \backslash\{a, b, c, d\}$ such that the sets of edges incident with branchvertices of $H$ are $\{a, b, p\},\{b, c, q\},\{c, d, r\}$ and $\{d, a, s\}$. Since every branch-vertex is incident with at least one edge in $X$, we may assume that $X=\{b, d\}$ or $\{b, c, d\}$. Since $a, p \notin X$, it follows that one is in $X_{1}$ and the other in $X_{2}$, say $a \in X_{1}$ and $p \in X_{2}$. Similarly, since $a, s \notin X$ it follows that $s \in X_{2}$. Let $P$ be the path in $L(H)$ between $p, r$ whose vertex set is the edge-set of the branch of $H$ containing $p, r$, and choose $Q$ containing $q, s$ similarly. Thus $P$ is odd, and so is $Q$. If they both have length 1 then $H$ has 6 vertices and the fourth outcome of the theorem holds. We may therefore assume that $P$ has length $\geq 3$. The path $b-p-P-r-d$ is odd and has length $\geq 5$; its ends are $Y$-complete and its internal vertices are not, so by $2.1, Y$ contains a leap. Hence there exist nonadjacent $y, y^{\prime} \in Y$ such that $y-r-P-p-y^{\prime}$ is a path in $G$. Since $p \in X_{2}$ and $y$ is nonadjacent to $p$ it follows that $y=y_{2}$; and since $s \in X_{2}$ and $y \neq y^{\prime}$, it follows that $y^{\prime}$ is adjacent to $s$. Now $y-r-P-p-y^{\prime}$ is an odd path, and it cannot be completed to an odd hole, so $y, y^{\prime}$ have no common neighbour in 
$Q$. But $b-q-Q-s-d$ is an odd path; its ends are $\left\{y, y^{\prime}\right\}$-complete, and its internal vertices are not, so by 2.1, $y, y^{\prime}$ form a leap for this path, that is, $y-q-Q-s-y^{\prime}$ is a path of $G$. (Note that this holds even if $b-q-Q-s-d$ has length 3 , since the anticonnected set in question has cardinality 2.) Since $y^{\prime}$ is major and nonadjacent to $q$ it follows that $y^{\prime}$ is adjacent to $c$, and similarly $y$ is adjacent to $a$. But then the fifth outcome of the theorem holds. This proves (1).

In the arguments to come there is a certain amount of moving from $H$ to $L(H)$ and back, and to facilitate this, for every subgraph $H^{\prime}$ of $H$ we denote by $L\left(H^{\prime}\right)$ the induced subgraph of $L(H)$ formed by the edges of $H^{\prime}$. So for any track $P$ of $H, L(P)$ is a path of $L(H)$. We say a branch-vertex $b$ of $H$ is a triad if $b$ is incident with at most one edge in $X$. It follows that every triad has degree 3 in $H$, and is incident with exactly one edge in each of $X, X_{1}, X_{2}$.

We recall that $Y$ is the vertex set of an antipath between $y_{1}, y_{2}$; let $Q$ be this antipath. There are two cases, depending whether $Q$ is odd or even.

(2) If $Q$ is odd then there is no cycle of $H$ with edge-set $\left\{h_{1}, h_{2}, h_{3}, h_{4}\right\}$ in order, such that the common end of $h_{1}$ and $h_{2}$ is a branch-vertex, $h_{1} \in X_{1}, h_{2} \in X_{2}$, and $h_{3}, h_{4} \in X$.

For if there is such a cycle, then $Q$ can be completed to an odd antihole via $y_{2}-h_{2}-h_{4}-f-h_{3}-h_{1}-y_{1}$ (where $f$ is a third edge of $H$ such that $h_{1}, h_{2}, f$ have a common end), a contradiction. This proves $(2)$.

(3) If $Q$ is odd and $h_{1} \in X_{1}$ meets $h_{2} \in X_{2}$, then every edge in $X$ meets at least one of $h_{1}, h_{2}$.

For if $h_{1} \in X_{1}$ meets $h_{2} \in X_{2}$, and $f \in X$ meets neither of $h_{1}, h_{2}$, then $Q$ can be completed to an odd antihole via $y_{2}-h_{2}-f-h_{1}-y_{1}$, a contradiction. This proves (3).

There is a branch-vertex $b$ of $H$ incident with at least two edges not in $X$. For $i=1,2$ let $e_{i} \in X_{i}$ be incident with $b$, and let $e_{3}$ be some third edge incident with $b$. For $i=1,2,3$, let $B_{i}$ be the branch of $H$ containing $e_{i}$, and let $b_{i}$ be its other end. If $Q$ is odd, let $f_{i} \in X$ be incident with $b_{i}$, chosen in addition such that $f_{i} \notin E\left(B_{i}\right)$ if possible $\left(1 \leq i \leq 3\right.$ ). (If $Q$ is even we choose the $f_{i}$ 's a little differently, described later.)

(4) If $Q$ is odd then $b_{3}$ is a triad.

For suppose not; then $f_{3} \notin E\left(B_{3}\right)$, and there is a second edge $f_{3}^{\prime} \in X$ incident with $b_{3}$. By (3), the edge $f_{3}$ meets one of $e_{1}, e_{2}$, and from the symmetry we may assume that it meets $e_{1}$. Thus $f_{3}=b_{1} b_{3}$ and $E\left(B_{1}\right)=\left\{e_{1}\right\}$. Since $H$ is bipartite, it follows that $B_{3}$ is even. Thus $f_{3}^{\prime}$ is not incident with $b$, and by (3) applied to $f_{3}^{\prime}, e_{1}$ and $e_{2}$ we deduce that $f_{3}^{\prime}=b_{2} b_{3}$ and $E\left(B_{2}\right)=\left\{e_{2}\right\}$. But the edges $e_{1}, e_{2}, f_{3}^{\prime}, f_{3}$ contradict (2). This proves (4).

(5) If $Q$ is odd and either $B_{3}$ has length $>1$ or $b$ is not a triad, then the theorem holds.

For assume that $B_{3}$ has length $\geq 2$. By (3) applied to $e_{3}$ and the two edges of $E(H) \backslash X$ incident with $b_{3}$ it follows that $B_{3}$ has length two and $f_{3} \notin E\left(B_{3}\right)$. (Later we will use the shorthand "by (3) applied to $e_{3}$ and $b_{3}$ ".) By (3) applied to $f_{3}, e_{1}$ and $e_{2}$ we deduce that $f_{3}$ is incident with 
$e_{1}$ or $e_{2}$, and so from the symmetry we may assume that $f_{3}=b_{1} b_{3}$ and $E\left(B_{1}\right)=\left\{e_{1}\right\}$. Suppose that $B_{2}$ has length at least two. By (3) applied to $f_{2}, e_{1}$ and $e_{2}$, it follows that either $f_{2}=b_{1} b_{2}$ or $E\left(B_{2}\right)=\left\{e_{2}, f_{2}\right\}$; and therefore in both cases $b_{2}, b_{3}$ are nonadjacent, since $H$ is bipartite. But this contradicts (3) applied to $f_{2}$ and $b_{3}$. It follows that $B_{2}$ has length 1 , and $E\left(B_{2}\right)=\left\{e_{2}\right\}$. From (3) applied to $f_{2}$ and $b_{3}$ we deduce that $b_{2}$ is adjacent to $b_{3}$ and $b_{2} b_{3} \notin X$. The vertex $b$ has degree 3 , for a fourth edge incident with $b$ would violate (3) applied to that edge and $b_{3}$. Since $H$ is cyclically 3 -connected, it follows that $H$ is the union of $B_{1}, B_{2}, B_{3}$, the edges $b_{1} b_{3}, b_{2} b_{3}$ and a branch $B$ with ends $b_{1}$ and $b_{2}$. The branch $B$ includes $f_{2}$, and its edge incident with $b_{1}$, say $e$, is not in $X$ by (3) applied to $e$ and $b_{3}$. But $e$ meets $f_{2}$, by (3) applied to $f_{2}$ and $b_{1}$. Thus $B$ has length two, and hence the fourth outcome of the theorem holds. We may therefore assume that $E\left(B_{3}\right)=\left\{e_{3}\right\}$. In this case $e_{3}$ is the only member of $X$ incident with $b_{3}$, and from (4) with $b, b_{3}$ exchanged it follows that $b$ is a triad. This proves (5).

(6) If $Q$ is odd and one of $B_{1}, B_{2}$ has length $>1$ then the theorem holds.

For suppose first that they both have length at least two. Then, for $i=1,2$, by (3) applied to $b$ and $f_{i}$ we deduce that $E\left(B_{i}\right)=\left\{e_{i}, f_{i}\right\}$ and therefore $f_{i}$ is the unique edge of $X$ incident with $b_{i}$. This contradicts (3) applied to $f_{1}$ and $b_{2}$. So at least one of $B_{1}, B_{2}$ has length 1 , and from the symmetry we may assume that $E\left(B_{1}\right)=\left\{e_{1}\right\}$ and $B_{2}$ has length at least two. If $f_{2} \in E\left(B_{2}\right)$ then $b_{2}$ is a triad, and the theorem holds by (5) with $b, b_{2}$ exchanged, so we may assume that $f_{2} \notin E\left(B_{2}\right)$. Let $e_{2}^{\prime}$ be the edge of $B_{2}$ incident with $b_{2}$. By (3) applied to $f_{2}$ and $b$ we deduce that $f_{2}=b_{1} b_{2}$, and that no edge incident with $b_{2}$ belongs to $X$ except $f_{2}$ and possibly $e_{2}^{\prime}$. By (3) and (4) applied to $f_{2}$ and $b_{3}$, it follows that $b_{3}$ is adjacent to $b_{2}$ and $b_{2} b_{3} \notin X$. Suppose for a contradiction that $b_{1}$ is not a triad, and choose $e_{1}^{\prime} \in X \backslash\left\{b_{1} b_{2}\right\}$ incident with $b_{1}$. By (3) applied to $e_{1}^{\prime}$ and $b_{2}$, it follows that $e_{2}^{\prime} \in X$, and from (3) applied to $e_{2}^{\prime}$ and $b$ we deduce that $E\left(B_{2}\right)=\left\{e_{2}, e_{2}^{\prime}\right\}$. But now the edges $e_{1}, e_{2}, e_{2}^{\prime}, f_{2}$ contradict (2). This proves that $b_{1}$ is a triad, and from (4) with $b, b_{1}$ exchanged, we deduce that $b_{2}$ is a triad. Since $H$ is cyclically 3 -connected, it follows that $H$ is the union of $B_{1}, B_{2}, B_{3}$, the edges $b_{1} b_{2}$ and $b_{2} b_{3}$ and a branch $B$ with ends $b_{1}$ and $b_{3}$. From (3) applied to $b_{1}$, we deduce that no edge of $B_{2}$ belongs to $X$, and by (3) applied to $b_{2}$ it follows that no edge of $B$ belongs to $X$. But then the theorem holds by (1). This proves (6).

\section{(7) If $Q$ is odd then the theorem holds.}

For by (5) and (6) we may assume that $E\left(B_{i}\right)=\left\{e_{i}\right\}$ for $i=1,2,3$. For $i=1,2$ let $f_{i}=b_{i} x_{i}$. Then $x_{1} \neq x_{2}$, for otherwise the edges $e_{1}, e_{2}, f_{2}, f_{1}$ violate (2). By (3) applied to $f_{i}$ and $b_{3}$ we deduce that $x_{i}$ is adjacent to $b_{3}$ and $x_{i} b_{3} \notin X$, and therefore $f_{i}$ is the unique edge in $X$ incident with $b_{i}$, and $b_{i}$ is a triad $(i=1,2)$. By $(3)$ applied to $f_{2}$ and $b_{1}$ we deduce that $b_{1}$ is adjacent to $x_{2}$, and, similarly, $x_{1}$ is adjacent to $b_{2}$. Since $H$ is a subdivision of a 3 -connected graph, $J=K_{3,3}$, and $L(H)$ is a degenerate appearance of $J$, and there is a $J$-enlargement that appears in $\bar{G}$, so the third outcome of the theorem holds. This proves (7).

In view of (7) we may henceforth assume that $Q$ is even.

(8) Every edge in $X_{1}$ meets every edge in $X_{2}$. 
For if $h_{1} \in X_{1}$ does not meet some $h_{2} \in X_{2}$, then $Q$ can be completed to an odd antihole via $y_{2}-h_{2}-h_{1}-y_{1}$, a contradiction. This proves (8).

A vertex of a track $P$ is penultimate if it is adjacent in $P$ to an end of $P$.

(9) For all $W \in\left\{X, X \cup X_{1}, X \cup X_{2}\right\}$ and for every even track $P$ in $H$ of length $\geq 4$ and with both end-edges and no internal edges in $W$, every edge in $W$ is incident with a penultimate vertex of $P$.

For let $f \in W$. If $W=X$ let $Y^{\prime}=Y$, and if $W=X \cup X_{i}$ where $i \in\{1,2\}$, let $Y^{\prime}=Y \backslash\left\{y_{i}\right\}$. So $W$ is the set of $Y^{\prime}$-complete vertices of $L(H)$. The path $L(P)$ of $G$ is odd and has length $\geq 3$; its ends are $Y^{\prime}$-complete, and its internal vertices are not. By $2.2, f$ is adjacent (in $G$ ) to vertices in the interior of $L(P)$; that is, $f$ is incident in $H$ with an internal vertex of $P$. We must show that $f$ is incident with a penultimate vertex. Let $P$ have vertices $p_{1}-\cdots-p_{n}$ and edges $h_{1}, \ldots, h_{n-1}$, where $h_{i}$ is incident with $p_{i}, p_{i+1}$ for $1 \leq i<n$; so $n$ is odd and $n \geq 5$. Suppose first that both ends of $f$ belong to $P$, say $f=p_{i} p_{j}$ where $i<j$. Since $H$ is bipartite, $j-i$ is odd, and so either $i-1$ or $n-j$ is odd, and from the symmetry we may assume the former, that is, $i$ is even. Hence the track $T$ with edge-set $\left\{h_{1}, \ldots, h_{i-1}, f\right\}$ has even length, at least 4 (since we may assume that $i \neq 2$ ); and yet in $G$ the $Y^{\prime}$-complete vertex $h_{n-1}$ has no neighbour in the interior of the odd path $L(T)$, contrary to 2.2. So not both ends of $f$ belong to $P$. Hence $f$ is incident with a unique vertex $p_{i}$ of $P$, and again we may assume that $3 \leq i \leq n-2$. In $G, h_{1^{-}} \cdots-h_{i-1^{-}} f$ is a path; its ends are $Y^{\prime}$-complete, and its internal vertices are not, and the $Y^{\prime}$-complete vertex $h_{n-1}$ has no neighbour in its interior; so by 2.2 , this path is even, that is, $i$ is odd. Since $p_{i}$ is a branch-vertex of $H$, and at least two of the edges incident with it do not belong to $W$, it follows that $W=X$ and $Y^{\prime}=Y$; and we may assume that $h_{i-1} \in X_{1}$ and $h_{i} \in X_{2}$. Since every edge in $X_{1}$ meets every edge in $X_{2}$, it follows that $h_{1}, \ldots, h_{i-2} \notin X_{1}$. In $G$, the path $h_{1}-\cdots-h_{i-1}$ is odd; its ends are $Y$-complete, its internal vertices are not, and the $Y$-complete vertex $h_{n-1}$ has no neighbour in its interior, so it has length 1 , that is, $i=3$. Similarly $n-i=2$, that is, $n=5$. But then $Q$ can be completed to an odd antihole via $y_{2}-h_{3}-h_{1}-h_{4}-h_{2}-y_{1}$, a contradiction. This proves (9).

(10) If $P_{1}, P_{2}, P_{3}$ are tracks in $H$ with a common end $v$, say, and otherwise vertex-disjoint, each with an edge in $X$, then at least two of the three edges of $P_{1} \cup P_{2} \cup P_{3}$ incident with $v$ belong to $X$.

For we may assume that for $i=1,2,3, P_{i}$ is between $v$ and $v_{i}$ say, and the only edge of $P_{i}$ in $X$ is the edge incident with $v_{i}$. Some two of $P_{1}, P_{2}, P_{3}$ have lengths of the same parity, say $P_{1}, P_{2}$, and so $P_{1} \cup P_{2}$ is a track of even length. If it has length 2 then $P_{1}, P_{2}$ both have length 1 and the claim holds, so we assume it has length $\geq 4$. The edge of $P_{3}$ incident with $v_{3}$ is incident with a penultimate vertex of this track, by (9), and so $P_{3}$ and one of $P_{1}, P_{2}$ have length 1 , and again the claim holds. This proves (10).

Earlier (preceding (4)) we chose $b$ such that at least two edges of $H$ incident with $b$ did not belong to $X$. Let us refine this choice; now in addition we choose $b$ such that $B_{3}$ is as long as possible.

(11) For $i=1,2$ there is an edge $f_{i} \in X$ incident with $b_{i}$ that does not meet $e_{3}$. 
For it suffices to prove this for $i=1$, and it clearly holds if there are at least two members of $X$ incident with $b_{1}$. So we may assume that there is a unique member of $X$ incident with $b_{1}$, and that this edge meets $e_{3}$, and therefore is the edge $b_{1} b_{3}$. But then $b_{1}$ is a triad, and $E\left(B_{3}\right)=\left\{e_{3}\right\}$, and $\left|E\left(B_{1}\right)\right|>1$, because $H$ is bipartite. The unique edge of $X_{1}$ incident with $b_{1}$ meets $e_{2}$ by (8); and hence this edge is $b_{1} b_{2}$, and $e_{2}=b b_{2}$. Suppose for a contradiction that there is a fourth edge $b v$ incident with $b$, and let $f$ be an edge incident with $b_{3}$ different from $b b_{2}, b_{1} b_{2}$; then $v \neq b, b_{1}, b_{2}, b_{3}$, and there is a track of length 4 with vertices $b_{3}-b_{1}-b_{2}-b-v$ in order; its end-edges belong to $X$ and its internal edges do not; and $f \in X$ is not incident with any penultimate vertex of this track, contrary to (9). This proves that $b$ has degree three. Since $H$ is cycically 3 -connected, it follows that $H$ consists of $B_{1}, B_{2}, B_{3}$, the edges $b_{1} b_{2}, b_{1} b_{3}$, and a branch $B$ with ends $b_{2}$ and $b_{3}$ that includes a member of $X$ incident with $b_{2}$. Since $H$ is bipartite, it follows that $|E(B)|>1$, and hence $b_{2}$ and $B$ contradict the choice of $b$ and $B_{3}$. This proves (11).

(12) If there exist $f_{1}, f_{2}$ as in (11) with $f_{1}, f_{2} \neq b_{1} b_{2}$ then the theorem holds.

For it follows from (10) applied to subtracks of the tracks with edge-sets $E\left(B_{1}\right) \cup\left\{f_{1}\right\}, E\left(B_{2}\right) \cup\left\{f_{2}\right\}$ and $\left\{e_{3}\right\}$ that $B_{1}, B_{2}$ include no member of $X$, and that $f_{1}$ meets $f_{2}$. Thus $b_{1}$ is not adjacent to $b_{2}$. We claim that for $i=1,2$ the edge $f_{i}$ is the only edge of $X$ incident with $b_{i}$. For suppose that say $f_{1}^{\prime} \in X$ is incident with $b_{1}$. By (10) applied to the vertex $b$ and the tracks with edge-sets $E\left(B_{1}\right) \cup\left\{f_{1}^{\prime}\right\}, E\left(B_{2}\right) \cup\left\{f_{2}\right\}$ and $\left\{e_{3}\right\}$, we deduce that $f_{1}^{\prime}$ meets $e_{3}$. Thus $B_{1}$ is even. Let $P$ be the track obtained from $B_{1}$ by adding $e_{3}$ and $f_{1}$; then $P$ and the edge $f_{2}$ violate (9). This proves our claim that $f_{i}$ is the only edge of $X$ incident with $b_{i}$ for $i=1,2$. Consequently, $b_{1}$ and $b_{2}$ are triads. From (8) we deduce that $B_{1}$ and $B_{2}$ have length one. For $i=1,2$ let $d_{i}$ be the edge incident with $b_{i}$ different from $e_{i}, f_{i}$; so $d_{1} \in X_{2}$ and $d_{2} \in X_{1}$. By (8) the edges $d_{1}, d_{2}$ meet; let $v$ denote their common end. Every edge $g$ incident with $v$ other than $d_{1}$ and $d_{2}$ belongs to $X$. If some such $g$ does not meet $e_{3}$ then the edges $g, d_{2}, e_{2}, e_{3}$ form a track with end-edges in $X$ and internal edges not in $X$, and $f_{1}$ is not incident with a penultimate vertex of this track, contrary to (9). So every such edge $g$ meets $e_{3}$ and hence is incident with $b_{3}$ (since $H$ is bipartite). Thus $v$ has degree two or three. If $v=b_{3}$, then $B_{3}$ has length 2 and both its edges belong to $X$, and the fourth outcome of the theorem holds. If $v \neq b_{3}$ and $v$ has degree 3 , then the third edge incident with $v$ is $v b_{3}$, and $b$ is a triad, and $H$ consists of the vertices $b, b_{1}, b_{2}, b_{3}, v$ and a branch $B$ with ends $b_{3}$ and $u$, where $u$ is the common end of $f_{1}$ and $f_{2}$; but then $J=K_{3,3}$, and if $B$ has length 1 then the second outcome of the theorem holds, and otherwise the first outcome holds. Finally, if $v \neq b_{3}$ and $v$ has degree two, then $b_{3}$ is the common end of $f_{1}, f_{2}$, and $J=K_{4}$ and the second outcome of the theorem holds. This proves (12).

From (11) and (12) we may therefore assume that $b_{1}, b_{2}$ are adjacent, and the edge $b_{1} b_{2} \in X$. From the symmetry we may assume that $B_{1}$ is even and $B_{2}$ is odd. Let $T$ be the track formed by $B_{1}$ and the edges $e_{3}, b_{1} b_{2}$. So $T$ is even. Suppose that there is an edge (say $f$ ) in $X$ incident with $b_{2}$ and different from $b_{1} b_{2}$. By (10) no edge of $B_{1}$ belongs to $X$, and yet $f$ is not incident with a penultimate vertex of $T$, contrary to (9). So there is no such edge $f$, and therefore $b_{2}$ is a triad. Let $e_{4}$ be the edge incident with $b_{2}$ different from $b_{1} b_{2}$ and not in $B_{2}$. So $e_{4} \in X_{1} \cup X_{2}$, and therefore by (8), $e_{4}$ meets one of $e_{1}, e_{2}$. Since it is not incident with $e_{1}$, it follows that $E\left(B_{2}\right)=\left\{e_{2}\right\}$, and $e_{4} \in X_{1}$. Let $B_{4}$ be the branch of $H$ containing $e_{4}$, and let $b_{4}$ be the other end of $B_{4}$.

(13) $b_{4}=b_{3}$, and $B_{3}$ has length 1 , and $H$ is a subdivision of $K_{4}$, and $B_{4}$ is even. 
For $b_{4}$ is different from $b, b_{1}, b_{2}$. Since $B_{1}$ is even, and $e_{2}$ is the unique edge in $X_{2}$ incident with $b$, it follows that no edge in $X_{2}$ incident with $b_{4}$ meets $e_{1}$, and therefore by (8), no edge in $X_{2}$ is incident with $b_{4}$. Consequently $b_{4}$ is not a triad, and so there are at least two edges (say $g_{1}, g_{2}$ ) in $X$ incident with $b_{4}$. By (10) (applied to three tracks with common end $b_{2}$ ), each of them meets either $b_{1} b_{2}$ or $e_{3}$. But no edge in $X$ is incident with both $b_{2}$ and $b_{4}$, since $e_{4} \in X_{1}$; so $g_{1}, g_{2}$ are either incident with $b_{1}$ or meet $e_{3}$.

Suppose that $b_{4}$ is not incident with $e_{3}$. Then at most one of $g_{1}, g_{2}$ is incident with $b_{1}$, and at most one meets $e_{3}$ (since $H$ is bipartite), so there is exactly one of each. Hence $b_{1}$ is adjacent to $b_{4}$, and $b_{1} b_{4} \in X$; and (since $H$ is bipartite and $B_{1}$ is even) $b$ is adjacent to $b_{4}$ and $b b_{4} \in X$, and $b_{4}$ has degree 3. Since $b_{4}$ is not incident with $e_{3}$, and $b_{4}$ is adjacent to $b$, it follows that $b_{4} \neq b_{3}$; and since $H$ is cyclically 3 -connected and $b_{2}$ is a triad, this is impossible. So $b_{4}$ is incident with $e_{3}$, that is, $b_{4}=b_{3}$ and $B_{3}$ has length 1 . Since this holds for every choice of $e_{3}$, we deduce that $b$ has degree 3 , and therefore $H$ is a subdivision of $K_{4}$. It follows that $B_{4}$ is even. This proves (13).

Let $B_{5}$ be the branch of $H$ between $b_{1}, b_{3}$. Since no edge incident with $b_{3}$ meets $e_{1}$ except $e_{3}$, it follows that $b_{3}$ is not a triad. Suppose that no edge of $B_{1}$ is in $X$. Then by (9) applied to $T$, every edge in $X$ is incident with one of $b, b_{1}$. In particular, no edge of $B_{4}$ is in $X \mathrm{i}$; and since $b_{3}$ is not a triad, it follows that $B_{5}$ has length 1 and its edge is in $X$. Thus $b_{3}$ is adjacent to both $b, b_{1}$, and the edges $b b_{3}, b_{1} b_{3}$ both belong to $X$; but then the theorem holds by (1).

So we may assume that some edge of $B_{1}$ is in $X$. This edge is not incident with a penultimate vertex of the track formed by $B_{4}$ and the edges $b_{1} b_{2}, e_{3}$, so by (9), some edge of $B_{4}$ belongs to $X$. By (10) applied to $B_{1}$, a subtrack of $B_{2} \cup B_{4}$ and the track consisting of the edge $e_{3}$, we deduce that the only edge of $B_{4}$ in $X$ is the edge incident with $b_{3}$. By (10) applied to the track with edge-set $E\left(B_{2}\right) \cup\left\{b_{1} b_{2}\right\}$, a subtrack of $B_{1}$ and the track consisting of the edge $e_{3}$, we deduce that the only edge of $B_{1}$ in $X$ is the edge incident with $b_{1}$. But $B_{5}$ is odd, and if it has length $>1$ then the first outcome of the theorem holds. So we may assume that $b_{1} b_{3}$ is an edge. Now the tracks $B_{1}, B_{4}$ are even; their end-edges belong to $X \cup X_{1}$, and their other edges do not (by (8)), and $e_{3}$ is not incident with a penultimate vertex of these tracks; so by (9), $B_{1}$ and $B_{4}$ both have length 2 . But then the fourth outcome of the theorem holds. This proves 6.1 .

\section{Rung replacement}

Before we apply 6.1, let us simplify it a little. We can effectively eliminate the cases of $L(H)$ being overshadowed. We need a few lemmas.

7.1 Let $c_{1}, c_{2}$ be adjacent vertices of a 3 -connected graph $J$, and let $e, f$ be edges of $J$ incident with $c_{1}$ and different from $c_{1} c_{2}$. There are three tracks of $J$ from $c_{1}$ to $c_{2}$, pairwise vertex-disjoint except for their ends, and with first edges $c_{1} c_{2}, e, f$ respectively.

Proof. Since $J$ is 3-connected, if we delete from $J$ all edges incident with $c_{1}$ except $e$ and $f$, the graph we make is still 2-connected, and so it has a cycle containing $c_{1}$ and $c_{2}$. This proves 7.1.

A prism means a graph consisting of two vertex-disjoint triangles $\left\{a_{1}, a_{2}, a_{3}\right\},\left\{b_{1}, b_{2}, b_{3}\right\}$, and three paths $P_{1}, P_{2}, P_{3}$, where each $P_{i}$ has ends $a_{i}, b_{i}$, and for $1 \leq i<j \leq 3$ the only edges between 
$V\left(P_{i}\right)$ and $V\left(P_{j}\right)$ are $a_{i} a_{j}$ and $b_{i} b_{j}$. The three paths $P_{1}, P_{2}, P_{3}$ are said to form the prism. The prism is long if at least one of the three paths has length $>1$.

7.2 Let $R_{1}, R_{2}, R_{3}$ form a prism in a Berge graph $G$; then $R_{1}, R_{2}, R_{3}$ all have the same parity.

The proof is clear.

7.3 Let $G$ be Berge, let $Y \subseteq V(G)$ be anticonnected, and for $i=1,2,3$ let $a_{i}-P_{i}-b_{i}$ be a path in $G \backslash Y$, forming a prism with triangles $\left\{a_{1}, a_{2}, a_{3}\right\},\left\{b_{1}, b_{2}, b_{3}\right\}$. Assume $P_{1}, P_{2}, P_{3}$ all have length $>1$, and that every vertex in $Y$ is adjacent to at least two of $a_{1}, a_{2}, a_{3}$ and to at least two of $b_{1}, b_{2}, b_{3}$. Then at least two of $a_{1}, a_{2}, a_{3}$ and at least two of $b_{1}, b_{2}, b_{3}$ are $Y$-complete.

Proof. Suppose not; then there is an antipath with interior in $Y$, joining two vertices either both in $\left\{a_{1}, a_{2}, a_{3}\right\}$ or both in $\left\{b_{1}, b_{2}, b_{3}\right\}$. Let $Q$ be the shortest such antipath. We may assume $Q$ joins $a_{1}$ and $a_{2}$ say. Since every vertex in $Y$ is adjacent to either $a_{1}$ or $a_{2}$ it follows that $Q$ has length $\geq 3$. From the minimality of $Q, a_{3}$ is $Q^{*}$-complete, and so is at least one of $b_{1}, b_{2}, b_{3}$, say $b_{i}$. Since $Q$ can be completed to an antihole via $a_{1}-b_{i}-a_{2}$ it follows that $Q$ is even. From 3.3 applied to the hole formed by $P_{1} \cup P_{2}$ and hat $a_{3}$, neither of $b_{1}, b_{2}$ is $Q^{*}$-complete, and so there is an antipath between $b_{1}$ and $b_{2}$ with interior in $Q^{*}$. By the minimality of $Q$, the two antipaths have the same interior; but this again contradicts 3.3. This proves 7.3.

In fact it is easy to find strengthenings of 7.3 in which some of the paths $P_{i}$ have length 1 , but for the moment 7.3 will suffice.

7.4 Let $G$ be Berge, and for $1 \leq i \leq 3$ let $P_{i}$ be a path of even length $\geq 2$, from $a_{i}$ to $b_{i}$, such that these three paths form a prism with triangles $A=\left\{a_{1}, a_{2}, a_{3}\right\}$ and $B=\left\{b_{1}, b_{2}, b_{3}\right\}$. Let $P_{1}^{\prime}$ be a path from $a_{1}^{\prime}$ to $b_{1}$, such that $P_{1}^{\prime}, P_{2}, P_{3}$ also form a prism. Let $y \in V(G)$ have at least two neighbours in $A$ and in $B$. Then it also has at least two neighbours in $\left\{a_{1}^{\prime}, a_{2}, a_{3}\right\}$.

Proof. Suppose not. By 7.2 $P_{1}^{\prime}$ has even length. Let $X$ be the set of neighbours of $Y$ in $G$. Then $a_{1}^{\prime} \notin X$, and $a_{1} \in X$, and exactly one of $a_{2}, a_{3} \in X$, say $a_{2} \in X$. Also, $y$ cannot be linked onto the triangle $A^{\prime}=\left\{a_{1}^{\prime}, a_{2}, a_{3}\right\}$, by 2.4 , and since one of $b_{2}, b_{3} \in X$ it follows that no internal vertex of $P_{1}^{\prime}$ is in $X$. Hence $b_{1} \notin X$, for otherwise $y$ - $a_{2}-a_{1}^{\prime}-P_{1}^{\prime}-b_{1}$ - would be an odd hole. So $b_{2}, b_{3} \in X$. Since $y-a_{1}-a_{3}-P_{3}-b_{3}-y$ is not an odd hole, there is a member of $X$ in $P_{3} \backslash b_{3}$. But then $y$ can be linked onto $A^{\prime}$, via $b_{2}-b_{1}-P_{1}^{\prime}-a_{1}$, the path $a_{2}$, and the path between $y$ and $a_{3}$ with interior in $V\left(P_{3}\right) \backslash\left\{b_{3}\right\}$, contrary to 2.4. This proves 7.4.

We shall only need the following when $J=K_{4}$ or $K_{3,3}$, but we might as well prove it in general.

7.5 Let $G$ be Berge, and let $L(H)$ be an overshadowed appearance of $J$ in $G$, where $J$ is 3-connected. Then either:

- there is a J-enlargement with a nondegenerate appearance in $G$, or

- $G$ admits a balanced skew partition. 
Proof. For each edge $u v$ of $J$, let $B_{u v}$ be the branch of $H$ with ends $u, v$, and let $R_{u v}$ be the path $L\left(B_{u v}\right)$ of $L(H)$. For each $v \in V(J)$ let $N_{v}$ be the clique of $L(H)$ with vertex set $\delta_{H}(v)$. There is an edge $c_{1} c_{2}$ of $J$ such that $B_{c_{1} c_{2}}$ has odd length $\geq 3$, and some vertex of $G$ is nonadjacent in $G$ to at most one vertex of $N_{c_{1}}$ and to at most one vertex of $N_{c_{2}}$. We say such a vertex $v$ is $B_{c_{1} c_{2}}$-dominant with respect to $L(H)$. Let the ends of $R_{c_{1} c_{2}}$ (that is, the end-edges of $B_{c_{1} c_{2}}$ ) be $r_{1}, r_{2}$, where $r_{i} \in N_{c_{i}}$. Let $Y$ be a maximal anticonnected set of vertices each with at most one non-neighbour in $N_{c_{1}}$ and at most one non-neighbour in $N_{c_{2}}$. We shall prove that $Y$ and some of its common neighbours separate the interior of $R_{c_{1} c_{2}}$ from the remainder of $L(H)$ in $G$, so that will be the skew partition we are looking for. Let $X$ be the set of all $Y$-complete vertices in $G$.

(1) For $i=1,2$, at most one vertex of $N_{c_{i}}$ is not in $X$.

For let $a_{1}, a_{2}$ be any two distinct vertices in $N_{c_{1}} \backslash\left\{r_{1}\right\}$; we shall show that at most one of $a_{1}, a_{2}, r_{1}$ is not in $X$. By 7.1, there are two paths $Q_{1}, Q_{2}$ of $H$ between $c_{1}$ and $c_{2}$, such that $Q_{1}, Q_{2}, B_{c_{1} c_{2}}$ are vertex-disjoint except for their ends, and for $i=1,2, a_{i}$ is the first edge of $Q_{i}$. Let $b_{i}$ be the other end-edge of $Q_{i}$. Both $Q_{1}$ and $Q_{2}$ have odd length, since $B_{c_{1} c_{2}}$ is odd and $H$ is bipartite; and they have length $\geq 3$ since $c_{1}, c_{2}$ are nonadjacent (for they are the ends of a branch of length $>1$.) Hence there are two paths $P_{1}, P_{2}$ of $L(H)$ from $N_{c_{1}}$ to $N_{c_{2}}$, such that $P_{1}, P_{2}, R_{c_{1} c_{2}}$ are vertex-disjoint and form a prism, and $P_{i}$ is from $a_{i}$ to $b_{i}$. Now $B_{c_{1} c_{2}}$ is odd and therefore $R_{c_{1} c_{2}}$ is even, and similarly $P_{1}$ and $P_{2}$ are even. By hypothesis, each member of $Y$ is adjacent to at least two vertices of the triangle $\left\{a_{1}, a_{2}, r_{1}\right\}$ and to two vertices of the triangle $\left\{b_{1}, b_{2}, r_{2}\right\}$. By 7.3 it follows that $X$ contains at least two members of $\left\{a_{1}, a_{2}, r_{1}\right\}$. This proves (1).

Let

$$
\begin{aligned}
X_{1} & =X \cap\left(N_{c_{1}} \cup N_{c_{2}}\right) \\
X_{2} & =X \cap\left(V(L(H)) \backslash\left(N_{c_{1}} \cup N_{c_{2}}\right)\right) \\
X_{0} & =X \backslash V(L(H)) \\
S & =V\left(R_{c_{1} c_{2}}\right) \backslash X_{1} \\
T & =\left(V(L(H)) \backslash V\left(R_{c_{1} c_{2}}\right)\right) \backslash X_{1} .
\end{aligned}
$$

We observe first that no vertex of $S$ is adjacent to any vertex in $T$; for such an edge would join two vertices both in $N_{c_{i}}$ for some $i$, and therefore both not in $X$, contradicting (1).

(2) If $F \subseteq V(G)$ is connected and some vertex of $S$ has a neighbour in $F$, and so does some vertex of $T$, and $F \cap\left(X_{0} \cup X_{1} \cup Y\right)=\emptyset$, then the theorem holds.

We shall prove this by induction on $|F|$; so, we assume it holds for all smaller choices of $F$ (even for different choices of $L(H)$ ). Hence we may assume that $G \mid F$ is a path with vertices $f_{1}, \ldots, f_{n}$ say, where $f_{1}$ is the only vertex of $F$ with a neighbour in $S$, and $f_{n}$ is the only vertex with a neighbour in $T$. From the minimality of $F$ it also follows that $F$ is disjoint from $L(H)$; for any vertex of $F$ in $L(H)$ would be in $S$ or $T$, since it is not in $X_{1}$, and then we could make $F$ shorter by omitting this vertex. Consequently $F \cap X=\emptyset$. Suppose some vertex in $v \in F$ is major with respect to $L(H)$. Then since $v \notin X$ it follows that $v$ has a nonneighbour in $Y$, and so $Y \cup v$ is anticonnected; the maximality of $Y$ therefore implies that $v \in Y$, and hence $F \cap Y \neq \emptyset$, a contradiction. So we may 
assume that no vertex in $F$ is major. On the other hand, the set of attachments of $F$ in $L(H)$ is not local, because it has an attachment in $R_{c_{1} c_{2}}$, and its attachments are not all contained in any of $V\left(R_{c_{1} c_{2}}\right), N_{c_{1}}, N_{c_{2}}$. Let us apply 5.8. Suppose first that 5.8.1 holds. Then we obtain an appearance $L\left(H^{\prime}\right)$ in $G$ of some $J$-enlargement, with $L(H)$ an induced subgraph of $L\left(H^{\prime}\right)$. Since $R_{c_{1} c_{2}}$ has even nonzero length, it follows that $L(H)$ is not degenerate, and therefore neither is $L\left(H^{\prime}\right)$, and hence the theorem holds. So we may assume that 5.8.2 holds, and there is an edge $b_{1} b_{2}$ of $J$, (for $i=1,2, s_{i}$ denotes the unique vertex in $N_{b_{i}} \cap R_{b_{1} b_{2}}$ ) and a path $P$ of $G$ with $V(P) \subseteq F$ and with ends $p_{1}$ and $p_{2}$, such that one of the following holds:

1. $p_{1}$ is adjacent in $G$ to all vertices in $N_{b_{1}} \backslash\left\{s_{1}\right\}$, and $p_{2}$ has a neighbour in $R_{b_{1} b_{2}} \backslash s_{1}$, and every edge from $V(P)$ to $V(L(H)) \backslash\left\{s_{1}\right\}$ is either from $p_{1}$ to $N_{b_{1}} \backslash\left\{s_{1}\right\}$, or from $p_{2}$ to $R_{b_{1} b_{2}} \backslash s_{1}$, or

2. for $i=1,2, p_{i}$ is adjacent in $G$ to all vertices in $N_{b_{i}} \backslash\left\{s_{i}\right\}$, and there are no other edges between $V(P)$ and $V(L(H))$ except possibly $p_{1} s_{1}, p_{2} s_{2}$, and $P$ has the same parity as $R_{b_{1} b_{2}}$, or

3. $p_{1}=p_{2}$, and $p_{1}$ is adjacent to all vertices in $\left(N_{b_{1}} \cup N_{b_{2}}\right) \backslash\left\{s_{1}, s_{2}\right\}$, and all neighbours of $p_{1}$ in $V(L(H))$ belong to $N_{b_{1}} \cup N_{b_{2}} \cup R_{b_{1} b_{2}}$, and $R_{b_{1} b_{2}}$ is even, or

4. $s_{1}=s_{2}$, and for $i=1,2, p_{i}$ is adjacent in $G$ to all vertices in $N_{b_{i}} \backslash\left\{s_{i}\right\}$, and there are no other edges between $V(P)$ and $V(L(H)) \backslash\left\{s_{1}\right\}$, and $P$ is even.

In case 1 , let $R^{\prime}$ be the (unique) path from $p_{1}$ to $s_{2}$ in $\left(V(P) \cup V\left(R_{b_{1} b_{2}}\right)\right) \backslash\left\{s_{1}\right\}$, and in the other cases let $R^{\prime}$ be $P$. So if in $L(H)$ we replace $R_{b_{1} b_{2}}$ by $R^{\prime}$ we obtain another appearance of $J$ in $G$, say $L\left(H^{\prime}\right)$, where $H^{\prime}$ is obtained from $H$ by replacing the branch $B_{b_{1} b_{2}}$ by some new branch $B^{\prime}$ joining the same two vertices. For each $v \in V(J)$ let $N_{v}^{\prime}$ be the clique in $L\left(H^{\prime}\right)$ formed by the edges in $\delta_{H^{\prime}}(v)$. So $N_{v}^{\prime}=N_{v}$ for all vertices $v$ of $J$ except for $b_{1}$ and $b_{2}$. Let $R^{\prime}$ be between $r_{1}^{\prime}$ and $r_{2}^{\prime}$, where $r_{i}^{\prime} \in N_{b_{i}}^{\prime}$ for $i=1,2$.

Now suppose that $b_{1} b_{2}$ and $c_{1} c_{2}$ are different edges of $J$. Then $B_{c_{1} c_{2}}$ is still a branch of $H^{\prime}$, and we claim that every $y \in Y$ is $B_{c_{1} c_{2}}$-dominant with respect to $L\left(H^{\prime}\right)$. For let $e, f$ be two edges of $J$ incident with $c_{1}$ and different from $c_{1} c_{2}$. By 7.1 there are three tracks of $J$ from $c_{1}$ to $c_{2}$, vertexdisjoint except for their ends, and one of them is the edge $c_{1} c_{2}$, and the first edges of the other two are $e$ and $f$. There are three tracks corresponding to these in $H$, and their line graph is a prism in $L(H)$. There also correspond three tracks in $H^{\prime}$, yielding a prism in $L\left(H^{\prime}\right)$. Since $R_{b_{1} b_{2}} \neq R_{c_{1} c_{2}}$, it follows that $R_{b_{1} b_{2}}$ is incident with at most one of $c_{1}, c_{2}$, so these two prisms are related as in 7.4. Hence by 7.4, since $y$ has two neighbours in both triangles of the first prism, it also has two neighbours in the triangles of the second. This proves that $y$ is $B_{c_{1} c_{2}}$-dominant with respect to $L\left(H^{\prime}\right)$. The same argument in the reverse direction shows that $Y$ remains a maximal anticonnected set of $B_{c_{1} c_{2}}$-dominant vertices. Since there is a proper subset $F^{\prime}$ of $F$ with attachments in $S$ and in the new set $T^{\prime}$ in $V\left(H^{\prime}\right)$ corresponding to $T$ (for $T^{\prime}$ contains all the vertices of $R^{\prime}$ that are in $F$, and there is at least one such vertex), it follows that we may apply the inductive hypothesis. So $F^{\prime}$, and hence $F$, contains a vertex of $X$. This completes the argument when $b_{1} b_{2}$ and $c_{1} c_{2}$ are distinct edges.

Now we assume that $b_{i}=c_{i}$ for $i=1,2$. There were four cases in the definition of $P$, listed above. Case 3 is impossible, since then the vertex $p_{1}$ would be $B_{c_{1} c_{2}}$-dominant with respect to $L(H)$, and therefore would be in either $X$ or $Y$, a contradiction. Also, case 1 is impossible, by applying 7.4 as before to show that $Y$ remains a maximal anticonnected set of $B^{\prime}$-dominant vertices, and 
applying the inductive hypothesis. Case 4 is impossible since $B_{c_{1} c_{2}}$ has length $\geq 3$. So case 2 applies; that is, $p_{2}$ is adjacent to all vertices in $N_{c_{2}} \backslash\left\{r_{2}\right\}$, and to no vertex of $R_{c_{1} c_{2}}$ except possibly $r_{2}$. So $N_{c_{i}}^{\prime}=\left(N_{c_{i}} \backslash\left\{r_{i}\right\}\right) \cup\left\{r_{i}^{\prime}\right\}$ for $i=1,2$. We recall that in this case $R^{\prime}=P$, and $P$ is a subpath of the path with vertices $f_{1}, \ldots, f_{n}$. Choose $h$ with $1 \leq h \leq n$ minimum such that $f_{h}$ is a vertex of $R^{\prime}$. Since both $R^{\prime}$ and $G \mid F$ are paths it follows that $f_{h}$ is one end of $R^{\prime}$, say $r_{1}^{\prime}$. (This is without loss of generality, because in this case 2 , there is symmetry between $b_{1}=c_{1}$ and $b_{2}=c_{2}$.) From the minimality of $F, r_{1}^{\prime}$ has no neighbour in $T$, and in particular every vertex in $N_{c_{1}} \backslash\left\{r_{1}\right\}$ is in $X$. We claim also that every vertex of $N_{c_{2}} \backslash\left\{r_{2}\right\}$ is in $X$. For if not, then $r_{2} \in X$, and by 7.1 there is a prism $R_{c_{1} c_{2}}, P_{1}, P_{2}$ say, in $L(H)$, where each $P_{i}$ has an end $a_{i} \in N_{c_{1}}$ and an end $b_{i} \in N_{c_{2}}$, and $b_{2} \notin X$. (Consequently $r_{2}, b_{1} \in X$.) Hence at most one vertex of the triangle $\left\{r_{2}^{\prime}, b_{1}, b_{2}\right\}$ is in $X$, and some vertex in $X$ (namely $a_{1}$ ) has no neighbour in this triangle, so by 2.8, $Y$ cannot be linked onto this triangle. In particular, no vertex of $P_{2}$ is in $X$ except $a_{2}$. But then $a_{2}-P_{2}-b_{2}-r_{2}$ is an odd path between members of $X$, and none of its internal vertices are in $X$, and $a_{1}$ has no neighbour in its interior, contrary to 2.2. This proves that every vertex of $N_{c_{2}} \backslash\left\{r_{2}\right\}$ is in $X$. Consequently all vertices of $Y$ are $B^{\prime}$-dominant with respect to $L\left(H^{\prime}\right)$. We claim also that $Y$ is still maximal. For suppose not, and let $Y \subset Y^{\prime}$ for some larger anticonnected set $Y^{\prime}$ of $B^{\prime}$-dominant vertices. Since $r_{1}^{\prime}, r_{2}^{\prime}$ are not in $X$, they are certainly not $Y^{\prime}$-complete, and since by (1) applied to $Y^{\prime}$, at most one vertex of $N_{c_{i}}^{\prime}$ is not $Y^{\prime}$-complete for $i=1,2$, it follows that every vertex of $N_{c_{1}}^{\prime} \backslash\left\{r_{1}^{\prime}\right\}$ and $N_{c_{2}}^{\prime} \backslash\left\{r_{2}^{\prime}\right\}$ are $Y^{\prime}$-complete. But then all the members of $Y^{\prime}$ are $B_{c_{1} c_{2}}$-dominant with respect to $L(H)$, contrary to the maximality of $Y$. This proves that $Y$ is a maximal anticonnected set of $B^{\prime}$-dominant vertices with respect to $L\left(H^{\prime}\right)$. Hence we can apply induction on $F$, and the result follows. This proves (2).

It follows from (2) that there is a partition of $V(G) \backslash\left(X_{0} \cup X_{1} \cup Y\right)$ into two sets $L$ and $M$ say, where there is no edge between $L$ and $M$, and $S \subseteq L$ and $T \subseteq M$. So $\left(L \cup M, X_{0} \cup X_{1} \cup Y\right)$ is a skew partition of $G$. By 4.2 we may assume it is not loose, and so $X_{2}$ is empty; and we shall show it is balanced.

(3) For $i=1,2$, all vertices of $N_{c_{i}} \backslash\left\{r_{i}\right\}$ belong to $X_{1}$.

For suppose there is a vertex $n_{1}$ of $N_{c_{1}} \backslash\left\{r_{1}\right\}$ not in $X$. Therefore all other vertices of $N_{c_{1}}$ belong to $X$, and in particular, $r_{1} \in X$. Suppose no other vertex of $R_{c_{1} c_{2}}$ is in $X$; then $r_{2} \notin X$, so $X$ includes $N_{c_{2}} \backslash\left\{r_{2} i\right\}$. Choose any $n_{2} \in N_{c_{2}} \backslash\left\{r_{2}\right\}$, and any $n_{1}^{\prime} \in N_{c_{1}} \backslash\left\{r_{1}\right\}$ different from $n_{1}$. Then $r_{1}-R_{c_{1} c_{2}}-r_{2}-n_{2}$ is an odd path between $Y$-complete vertices, and none of its internal vertices are $Y$-complete, and yet $n_{1}^{\prime}$ has no neighbour in its interior, contrary to 2.2. This proves that some vertex of $R_{c_{1} c_{2}}$ different from $r_{1}$ is in $X$; yet $X_{2}$ is empty, so the interior of $R_{c_{1} c_{2}}$ contains no vertex in $X$. Consequently $r_{2} \in X$. Choose $n_{2} \in N_{c_{2}} \backslash\left\{r_{2}\right\}$ such that $N_{c_{2}} \backslash\left\{n_{2}\right\} \subseteq X$. Since $J$ is 3-connected, there is a track of $H$ from $c_{1}$ to $c_{2}$ with first edge $n_{1}$ and last edge different from $n_{2}$. This track is odd since $c_{1}$ and $c_{2}$ have opposite biparity; and so in $G$ there is an even path, $P$ say, from $n_{1}$ to some $n_{2}^{\prime} \in N_{c_{2}} \backslash\left\{n_{2}\right\}$, with no vertex in $N_{c_{1}} \cup N_{c_{2}}$ except its ends. But then $r_{1}-n_{1}-P-n_{2}^{\prime}$ is an odd path between $Y$-complete vertices, no vertex in its interior is $Y$-complete, and the $Y$-complete vertex $r_{2}$ has no neighbour in its interior, contrary to 2.2. This proves (3).

Let $W=\left(N_{c_{1}} \backslash\left\{r_{1}\right\}\right) \cup\left(N_{c_{2}} \backslash\left\{r_{2}\right\}\right)$. Then $W \subseteq X_{1}$ by (3), and since there are no edges between $N_{c_{1}}$ and $N_{c_{2}}$, it follows that $W$ has exactly two components, both cliques. In particular, $W$ is anticonnected. Now every $W$-complete vertex is $B_{c_{1} c_{2}}$-dominant, and so belongs to $X \cup Y$; and hence there are no $W$-complete vertices in $L \cup M$. Consequently $W$ is a kernel for the skew partition. 
Let us verify the hypotheses of 4.6. Suppose $u_{1}, u_{2} \in W$ are nonadjacent. Then one of $u_{1}, u_{2}$ is in $N_{c_{1}} \backslash\left\{r_{1}\right\}$ and the other in $N_{c_{2}} \backslash\left\{r_{2}\right\}$, and therefore they are joined by a path in $L(H)$ using no more vertices in $N_{c_{1}} \cup N_{c_{2}}$, which is even (since $H$ is bipartite). In particular, by $4.3, u_{1}, u_{2}$ are not joined by any odd path with interior in $L$. Finally, suppose there is a pair of vertices of $L$ joined by an odd antipath with interior in $W$, necessarily of length $\geq 5$ (since we already did the odd path case). Then $G \mid W$ contains an antipath of length 3, which is impossible since its components are cliques. From 4.6 it follows that $G$ admits a balanced skew partition. This proves 7.5.

\section{Generalized line graphs}

In this section we complete the proofs of 5.1 and 5.2. As we said earlier, our strategy is to find the biggest line graph in $G$ that we can, and then assemble all the alternative rungs for a given edge of $J$ into a "strip". Let us make that precise.

Let $J$ be 3-connected, and let $G$ be Berge. A $J$-strip system $(S, N)$ in $G$ means:

- for each edge $u v$ of $J$, a subset $S_{u v}=S_{v u} \subseteq V(G)$

- for each vertex $v$ of $J$, a subset $N_{v} \subseteq V(G)$

satisfying the following conditions (for $u v \in E(J)$, a $u v$-rung means a path $R$ of $G$ with ends $s, t$ say, where $V(R) \subseteq S_{u v}$, and $s$ is the unique vertex of $R$ in $N_{u}$, and $t$ is the unique vertex of $R$ in $\left.N_{v}\right)$ :

- The sets $S_{u v}(u v \in E(J))$ are pairwise disjoint

- For each $u \in V(J), N_{u} \subseteq \bigcup\left(S_{u v}: v \in V(J)\right.$ adjacent to $\left.u\right)$

- For each $u v \in E(J)$, every vertex of $S_{u v}$ is in a $u v$-rung

- If $u v, w x \in E(J)$ with $u, v, w, x$ all distinct, then there are no edges between $S_{u v}$ and $S_{w x}$

- If $u v, u w \in E(J)$ with $v \neq w$, then $N_{u} \cap S_{u v}$ is complete to $N_{u} \cap S_{u w}$, and there are no other edges between $S_{u v}$ and $S_{u w}$

- For each $u v \in E(J)$ there is a special $u v$-rung such that for every cycle $C$ of $J$, the sum of the lengths of the special $u v$-rungs for $u v \in E(C)$ has the same parity as $|V(C)|$.

It follows that for distinct $u, v \in V(J), N_{u} \cap N_{v}$ is empty if $u, v$ are nonadjacent, and otherwise $N_{u} \cap N_{v} \subseteq S_{u v}$; and for $u v \in E(J)$ and $w \in V(J)$, if $w \neq u, v$ then $S_{u v} \cap N_{w}=\emptyset$. The final axiom looks strange, but we shall show immediately that the same property holds for every choice of $u v$-rungs.

8.1 Let $(S, N)$ be a J-strip system in a Berge graph $G$, where $J$ is 3-connected. Then for every $u v \in E(J)$, all uv-rungs have lengths of the same parity.

Proof. Since $J$ is 3-connected, there is a cycle $C$ of $J$ with $|V(C)| \geq 4$ and with $u v \in E(C)$. For each $x y \in E(C)$ different from $u v$, choose an $x y$-rung $R_{x y}$. For every $u v$-rung $R$, the union of $V(R)$ and all the $V\left(R_{x y}\right)$ 's induces a cycle in $G$. This has length $\geq 4$ since $C$ has length $\geq 4$, so it is a hole and therefore even. Hence all choices of $R$ have lengths of the same parity. This proves 8.1. 
For each edge $u v$ of $J$, choose a $u v$-rung $R_{u v}$. It follows from 8.1 and the final axiom above that the subgraph of $G$ induced on the union of the vertex sets of these rungs is a line graph of a bipartite subdivision $H$ of $J$. For brevity we say that this choice of rungs forms $L(H)$.

We need two easy observations:

8.2 Let $(S, N)$ be a J-strip system in a Berge graph $G$, where $J$ is 3-connected. If there is an edge uv of $J$ such that some uv-rung has length 0 and another uv-rung has length $\geq 1$, then there is an overshadowed appearance of $J$ in $G$.

Proof. For each edge $i j$ of $J$ choose an $i j$-rung $R_{i j}$, arbitrarily for every edge of $J$ different from $u v$, and such that $R_{u v}$ has length $\geq 1$; and let this choice of rungs form $L(H)$. Let $y$ be the vertex of some $u v$-rung of length 0 . By 8.1, $R_{u v}$ has even length. Let $B$ be the branch of $H$ between $u$ and $v$, so $E(B)=V\left(R_{u v}\right)$. Then $B$ is odd and has length $\geq 3$ and $y$ is nonadjacent in $G$ to at most one vertex of $G$ in $\delta_{H}(u)$ and at most one in $\delta_{H}(v)$. Hence $L(H)$ is overshadowed. This proves 8.2.

A $J$-strip system is nondegenerate if there is some choice of rungs such that the line graph $L(H)$ they form is a nondegenerate appearance of $J$. 8.2 has the following corollary:

8.3 Let $(S, N)$ be a nondegenerate $J$-strip system in a Berge graph $G$, where $J$ is 3-connected. If there is no overshadowed appearance of $J$ in $G$, then for every choice of rungs, the line graph they form is a nondegenerate appearance of $J$ in $G$.

Proof. Take a choice of rungs $R_{i j}(i j \in E(J))$, forming $L(H)$ say, where $L(H)$ is nondegenerate; and suppose there is another choice, $R_{i j}^{\prime}(i j \in E(J))$, forming $L\left(H^{\prime}\right)$ say, where $L\left(H^{\prime}\right)$ is degenerate. Then for some $i j \in E(J), R_{i j}$ has nonzero length and $R_{i j}^{\prime}$ has length 0. By 8.2 there is an overshadowed appearance of $J$ in $G$. This proves 8.3.

Given a $J$-strip system $(S, N)$, we define $V(S, N)=\bigcup\left(S_{u v}: u v \in E(J)\right)$. Hence every $N_{v} \subseteq$ $V(S, N)$. If $u, v \in V(J)$ are adjacent, we define $N_{u v}=N_{u} \cap S_{u v}$. So every vertex of $N_{u}$ belongs to $N_{u v}$ for exactly one $v$. Note that $N_{u v}$ is in general different from $N_{v u}$, but $S_{u v}$ and $S_{v u}$ mean the same thing. We say $X \subseteq V(S, N)$ saturates the strip system if for every $u \in V(J)$, there is at most one neighbour $v$ of $u$ in $J$ such that $N_{u v} \nsubseteq X$; and a vertex $y \in V(G) \backslash V(S, N)$ is major (with respect to the strip system) if the set of its neighbours in $V(S, N)$ saturates $(S, N)$. We say $X \subseteq V(S, N)$ is local (with respect to the strip system) if either $X \subseteq N_{v}$ for some $v \in V(J)$, or $X \subseteq S_{u v}$ for some edge $u v \in E(J)$.

8.4 Let $G$ be Berge, and let $J$ be a 3-connected graph. Let $(S, N)$ be a J-strip system in $G$, nondegenerate if $J=K_{4}$. Let $y \in V(G) \backslash V(S, N)$, and let $X$ be the set of neighbours of $y$. If there is a choice of rungs forming a line graph that is saturated by $X$, then either:

- $X$ saturates the strip system, or

- there is a J-enlargement with a nondegenerate appearance in $G$, or

- $J=K_{4}$ and there is an overshadowed appearance of $J$ in $G$. 
Proof. We define the fork number of a choice of rungs to be the number of branch-vertices of $H$ incident in $H$ with $\geq 2$ edges in $X \cap E(H)$, where $L(H)$ is the line graph formed by this choice of rungs. Let us say that a choice of rungs $R_{i j}$ forming a line graph $L(H)$ is saturated if $X$ saturates $L(H)$, and in this case its fork number is $|V(J)|$. If every choice of rungs is saturated, then $X$ saturates the strip system as required, so we may therefore assume that there is some choice of rungs that is not saturated. Hence there are two choices of rungs $R_{i j}(i j \in E(J))$ and $R_{i j}^{\prime}(i j \in E(J))$, so that the first is saturated and the second is not, differing only on one edge of $J$. Let the line graphs made by $R_{i j}$ and $R_{i j}^{\prime}$ be $L(H)$ and $L\left(H^{\prime}\right)$ respectively.

Let us apply 5.7 to $H^{\prime}$ and $X \cap E\left(H^{\prime}\right)$. Then 5.7.1 is false; suppose that 5.7.6 holds. Then $G \mid\left(V\left(L\left(H^{\prime}\right)\right) \cup\{y\}\right)=L\left(H^{\prime \prime}\right)$ say, and $L\left(H^{\prime \prime}\right)$ is an appearance in $G$ of a $J$-enlargement. We may assume that $L\left(H^{\prime \prime}\right)$ is degenerate, for otherwise the theorem holds. Hence $J=K_{4}$ and $L\left(H^{\prime}\right)$ is degenerate. Since the strip system is nondegenerate, the result follows from 8.3. So we may assume that one of 5.7.2-5 holds. Hence the choice of rungs $R_{i j}^{\prime}(i j \in E(J))$ has fork number is $\leq 2$. Since the two choices of rungs $R_{i j}(i j \in E(J))$ and $R_{i j}^{\prime}(i j \in E(J))$ differ only on one edge of $J$, their fork numbers differ by at most 2 ; and so $|V(J)|=4$, and $J=K_{4}$.

Let $V(J)=\{1,2,3,4\}$, and $R_{i j} \neq R_{i j}^{\prime}$ only for the edge 1-2. Let the ends of each $R_{i j}$ be $r_{i j}$ and $r_{j i}$, where $\left\{r_{i j}: j \in\{1, \ldots, 4\} \backslash\{i\}\right\}$ is a triangle $T_{i}$ for each $i$. Similarly each $R_{i j}^{\prime}$ is between $r_{i j}^{\prime}$ and $r_{j i}^{\prime}$, where for each $i,\left\{r_{i j}^{\prime}: j \in\{1, \ldots, 4\} \backslash\{i\}\right\}$ is a triangle $T_{i}^{\prime}$. Since $X$ saturates $L(H)$, it has at least two members in each of $T_{1}, \ldots, T_{4}$; and since $X$ does not saturate $L\left(H^{\prime}\right)$, there is some $T_{i}^{\prime}$ containing at most one member of $X$. Since $T_{3}=T_{3}^{\prime}$ and $T_{4}=T_{4}^{\prime}$, we may assume that $\left|X \cap T_{1}\right|=2$ and $\left|X \cap T_{1}^{\prime}\right|=1$; and so $r_{1,2} \in X, r_{1,2}^{\prime} \notin X$, and exactly one of $r_{1,3}, r_{1,4} \in X$, say $r_{1,3} \in X$ and $r_{1,4} \notin X$.

Also, at least two vertices of $T_{3}$ and $T_{4}$ are in $X$, so there are at least two branch-vertices of $H^{\prime}$ incident in $H^{\prime}$ with more than one edge in $X$. By 5.7 applied to $H^{\prime}$, we deduce that 5.7 .5 holds, and so there is an edge $i j$ of $J$ such that $R_{i j}^{\prime}$ is even and

$$
\left(X \cap V\left(L\left(H^{\prime}\right)\right)\right) \backslash V\left(R_{i j}^{\prime}\right)=\left(T_{i}^{\prime} \cup T_{j}^{\prime}\right) \backslash V\left(R_{i j}^{\prime}\right) .
$$

In particular, $T_{i}^{\prime}$ and $T_{j}^{\prime}$ both contain at least two vertices in $X$, and so $i, j \geq 2$. Since $r_{1,3} \in X$ it follows that one of $i, j=3$, say $j=3$, and $r_{1,3} \in T_{3}$; so $R_{1,3}$ has length 0 . Now there are two cases, $i=2$ and $i=4$. Suppose first that $i=2$. Then

$$
\left(X \cap V\left(L\left(H^{\prime}\right)\right)\right) \backslash V\left(R_{2,3}\right)=\left\{r_{1,3}, r_{3,4}, r_{2,4}, r_{2,1}^{\prime}\right\},
$$

and since at least two vertices of $T_{4}$ are in $X$ it follows that $R_{2,4}, R_{3,4}$ both have length 0 , a contradiction since $R_{i j}^{\prime}=R_{2,3}$ is even. So $i=4$, and hence $R_{3,4}$ is even and

$$
\left(X \cap V\left(L\left(H^{\prime}\right)\right)\right) \backslash V\left(R_{3,4}\right)=\left\{r_{3,1}, r_{4,1}, r_{3,2}, r_{4,2}\right\} .
$$

Since the path $r_{3,2}-R_{2,3}-r_{2,3}-r_{2,4}-R_{2,4}-r_{4,2}$ can be completed to a hole via $r_{4,2}-r_{4,3}-R_{3,4}-r_{3,4}-r_{3,2}$, it follows that the first path is even, and so exactly one of $R_{2,3}, R_{2,4}$ is odd; and since the same path can be completed to a hole via $r_{4,2}-r_{4,1}-R_{1,4}-r_{1,4}-r_{1,3}-r_{3,2}$ it follows that $R_{1,4}$ is odd. Since one of $R_{2,3}, R_{2,4}$ is odd, they do not both have length 0 , and hence at most one of $r_{2,3}, r_{2,4} \in X$. Since $X$ saturates $L(H)$, it follows that exactly one of $r_{2,3}, r_{2,4} \in X$ (and hence one of $R_{2,3}, R_{2,4}$ has length 0 ), and also that $r_{2,1} \in X$. Since no vertex of $R_{1,2}^{\prime}$ is in $X$, this restores the symmetry between $T_{1}^{\prime}$ and $T_{2}^{\prime}$. 
Suppose that $R_{2,3}$ has length 0 . Then $R_{2,4}$ and $R_{1,2}$ are odd, and in particular $r_{2,1} \neq r_{1,2}$. If $r_{2,1}$ has no neighbour in $R_{1,2}^{\prime}$, then $y-r_{2,1}-r_{2,4}-r_{2,1}^{\prime}-R_{1,2}^{\prime}-r_{1,2}^{\prime}-r_{1,4}-R_{1,4}-r_{4,1}-y$ is an odd hole, a contradiction. So $r_{2,1}$ has a neighbour in $R_{1,2}^{\prime}$; but then $y$ can be linked onto the triangle $T_{1}^{\prime}$ via $R_{1,2}^{\prime}$ and $R_{1,4}$, contrary to 2.4. This proves that $R_{2,3}$ has length $\geq 1$. Hence $R_{2,3}$ has odd length and $R_{2,4}$ has length 0 , and consequently $R_{1,2}, R_{3,4}$ have even length and $R_{1,4}$ is odd. If $R_{3,4}$ has positive length then $L(H)$ is overshadowed (because of the vertex $y$ ), and so the theorem holds. We may therefore assume that $R_{3,4}$ has length 0 . If $r_{2,1} \neq r_{1,2}$ and $r_{2,1}$ has no neighbour in $R_{1,2}^{\prime}$, then $y-r_{2,1}-r_{2,4}-r_{2,1}^{\prime}-R_{1,2}^{\prime}-r_{1,2}^{\prime}-r_{1,3}-y$ is an odd hole, a contradiction; while if $r_{2,1} \neq r_{1,2}$ and $r_{2,1}$ has a neighbour in $R_{1,2}^{\prime}$, then then $y$ can be linked onto the triangle $T_{1}^{\prime}$ via $R_{1,2}^{\prime}$ and $R_{1,4}$, contrary to 2.4. So $r_{2,1}=r_{1,2}$. But then $L(H)$ is degenerate. Since the strip system is nondegenerate, it follows from 8.3 that there is an overshadowed appearance of $K_{4}$ in $G$. This proves 8.4.

A $J$-strip system $(S, N)$ in $G$ is maximal if there is no $J$-strip system $\left(S^{\prime}, N^{\prime}\right)$ in $G$ such that $V(S, N) \subset V\left(S^{\prime}, N^{\prime}\right)$, and $S_{u v}^{\prime} \cap V(S, N)=S_{u v}$ for every $u v \in E(J)$, and $N_{v} \subseteq N_{v}^{\prime}$ for every $v \in V(J)$. We need to analyze maximal strip systems. For an edge $u v \in E(J)$, we call the set $S_{u v}$ a strip of the strip system.

8.5 Let $G$ be Berge, let $J$ be a 3-connected graph, and let $(S, N)$ be a maximal $J$-strip system in $G$. Assume that there is no J-enlargement with a nondegenerate appearance in $G$. Assume moreover that if $J=K_{4}$ then $(S, N)$ is nondegenerate and there is no overshadowed appearance of $J$ in $G$. Let $F \subseteq V(G) \backslash V(S, N)$ be connected, so that no member of $F$ is major with respect to $(S, N)$. Then the set of attachments of $F$ in $V(S, N)$ is local.

Proof. Let $X$ be the set of attachments of $F$ in $V(S, N)$, and suppose for a contradiction that $X$ is not local. We may assume that $F$ is minimal (connected) with this property.

(1) For every choice of rungs, forming $L(H)$ say:

- for each $y \in F$, the set of neighbours of $y$ does not saturate $L(H)$, and

- if $J=K_{4}$ then $L(H)$ is not degenerate.

For no $y \in F$ is major with respect to the strip system, and no $J$-enlargement has a nondegenerate appearance in $G$, and if $J=K_{4}$ then there is no overshadowed appearance of $J$ in $G$, so the first claim follows from 8.4. For the second claim, assume $J=K_{4}$; then by hypothesis, the strip system is not degenerate, and the claim follows from 8.3. This proves (1).

(2) There is no $v \in V(J)$ such that $X \subseteq \bigcup\left(S_{u v}: u v \in E(J)\right)$.

For assume that $v$ is such a vertex. Consequently, for every vertex $w \in V(J)$ except at most one, only one strip meets both $N_{w}$ and $X$. Since $X$ is not local, there exists $x \in X \cap S_{u v} \backslash N_{v}$ for some edge $u v$ of $J$. Since $X \nsubseteq S_{u v}$, there exists $x^{\prime} \in X \cap S_{u^{\prime} v}$ for some edge $u^{\prime} v$ of $J$ with $u^{\prime} \neq u$. For $w \in V(J), x$ belongs to $N_{w}$ only if $w=u$, and $x^{\prime}$ belongs to $N_{w}$ only if $w \in\left\{v, u^{\prime}\right\}$; and since $x, x^{\prime}$ do not belong to the same strip it follows that $\left\{x, x^{\prime}\right\}$ is not local with respect to the strip system. Make a choice of rungs $R_{i j} i j \in E(J)$ such that $x \in V\left(R_{u v}\right)$ and $x^{\prime} \in V\left(R_{u^{\prime} v}\right)$, forming $L(H)$. Then $\left\{x, x^{\prime}\right\}$ is not local with respect to $L(H)$, so by (1) we can apply 5.8. Suppose that 5.8.1 holds. Then there is an appearance $L\left(H^{\prime}\right)$ in $G$ of some $J$-enlargement $J^{\prime}$, with $L(H)$ an induced subgraph 
of $L\left(H^{\prime}\right)$. Moreover, if $J^{\prime}=K_{3,3}$ then $J=K_{4}$, and so $L(H)$ is nondegenerate and therefore so is $L\left(H^{\prime}\right)$. Since $J^{\prime} \neq K_{4}$ it follows that $L\left(H^{\prime}\right)$ is nondegenerate, contrary to hypothesis. So 5.8 .1 does not hold, and therefore 5.8.2 holds. Since for every vertex $w \in V(J)$ except at most one, only one strip meets both $N_{w}$ and $X$, it follows that 5.8.2.a holds, and there is a branch $D$ of $H$ with an end $d$ such that $\delta_{H}(d) \backslash E(D)=(X \cap E(H)) \backslash E(D)$. Since $x$ and $x^{\prime}$ are disjoint edges in $X \cap E(H)$, they are not both incident with $d$, and so one of them is in $E(D \backslash d)$. The branch containing $x^{\prime}$ does not meet $x$, so $D$ is the branch between $u$ and $v$, and $d=v$. Hence $x^{\prime}$ is incident with $v$ in $H$, and $\delta_{H}(v) \subseteq X \cup E(D)$. Consequently, for all neighbours $w \neq u$ of $v$ in $J, X$ contains the vertex of $R_{v w}$ that belongs to $N_{v}$, and contains no other vertex of $R_{v w}$. This restores the symmetry between $u^{\prime}$ and the other neighbours of $v$ different from $u$; and since it holds for all choices of the rungs $R_{v w}$, we deduce that $X \backslash S_{u v}=N_{v} \backslash S_{u v}$. The minimality of $F$ implies that there is a path $P$ with $V(P)=F$, with ends $p_{1}, p_{2}$ such that $p_{1}$ is complete to $N_{v} \backslash N_{v u}$, and no other vertex of $P$ has any neighbours in $N_{v} \backslash N_{v u}$, and $p_{2}$ is adjacent to $x$, and no other vertex of $P$ has any neighbours in $S_{u v} \backslash N_{v}$. But then we can add $p_{1}$ to $N_{v}$ and $F$ to $S_{u v}$, contradicting the maximality of $(S, N)$. This proves (2).

Let $K=\left\{u v \in E(J): X \cap S_{u v} \neq \emptyset\right\}$.

(3) There are two disjoint edges in $K$.

For make a choice of rungs $R_{u v}(u v \in E(J))$ such that $X \cap V\left(R_{u v}\right) \neq \emptyset$ for each $u v \in K$, forming $L(H)$. If there are no two disjoint edges in $K$, then by (1) and 5.8, it follows that either $X \cap V(L(H))$ is local (with respect to $L(H)$ ) or 5.8.2.a holds, and in either case there is a branch $D$ of $H$ with an end $d$ such that every edge of $X \cap E(H)$ either is in $E(D)$ or is incident with $d$. In particular, every branch containing an edge of $X$ is incident with $d$, and so $d$ meets all edges of $J$ in $K$, contrary to (2). This proves (3).

From (3) it follows that there exists a 2-element subset of $X$ that is not local, and so from the minimality of $F$ it follows that $F$ is the vertex set of a path, say $f_{1}, \ldots, f_{n}$. Let us say a choice $R_{u v}(u v \in E(J))$ of rungs is broad if there are two disjoint edges $i j$ and $h k$ of $J$ such that $X$ meets both $R_{i j}$ and $R_{h k}$. From (3) there is a broad choice. We denote the ends of $R_{u v}$ by $r_{u v}$ and $r_{v u}$, where $r_{u v} \in N_{u}$ and $r_{v u} \in N_{v}$.

(4) For every broad choice of rungs $R_{u v}(u v \in E(J))$, there is a unique pair $(i, j)$ of adjacent vertices of $J$ such that:

- for every $w \in V(J)$ different from $j$ and adjacent to $i$ in $J, r_{i w} f_{1}$ is the unique edge of $G$ between $V\left(R_{i w}\right)$ and $F$,

- for every $w \in V(J)$ different from $i$ and adjacent to $j$ in $J, r_{j w} f_{n}$ is the unique edge of $G$ between $V\left(R_{j w}\right)$ and $F$,

- for every edge uv of $J$ disjoint from ij, there are no edges of $G$ between $V\left(R_{u v}\right)$ and $F$.

For by (1) we can apply 5.8, and since the choice of rungs is broad, the minimality of $F$ implies that one of 5.8.2.b, 5.8.2.c, 5.8.2.d holds. Hence there is an edge $i j$ as in (4). Suppose there is another, say $i^{\prime} j^{\prime}$. Since $i^{\prime} j^{\prime}$ meets all edges of $J$ that share exactly one end with $i j$, and $J$ is 3 -connected, it 
follows that $J=K_{4}$ and the two edges $i j, i^{\prime} j^{\prime}$ are disjoint. Moreover, the unique vertex of $R_{i i^{\prime}}$ in $X$ is both $r_{i i^{\prime}}$ and $r_{i^{\prime} i}$, so $R_{i i^{\prime}}$ has length 0 . Similarly $R_{i j^{\prime}}, R_{j i^{\prime}}, R_{j j^{\prime}}$ all have length 0 , and so $L(H)$ is degenerate, contrary to (1). This proves (4).

\section{(5) Every choice of rungs is broad.}

For from (3), there is a broad choice, and from (4) in any broad choice $R_{u v}(u v \in E(J))$ there are four different edges $a_{1} b_{1}, \ldots, a_{4} b_{4}$ of $J$, such that $a_{1} b_{1}$ is disjoint from $a_{2}$, and $a_{3} b_{3}$ is disjoint from $a_{4} b_{4}$, and $X$ meets $R_{a_{i} b_{i}}$ for $1 \leq i \leq 4$. Consequently, if we take another choice of rungs, differing from this one on only one edge, then it too is broad. It follows that every choice is broad. This proves (5).

For a given choice of rungs, let us call the edge $i j$ as in (4) the traversal for the choice.

\section{(6) There are two choices of rungs with different traversals.}

Take a choice of rungs, and let $i j$ be its traversal; and suppose that all other choices of rungs have the same traversal. Let $A_{1}=N_{i} \backslash S_{i j}$, and $A_{2}=N_{j} \backslash S_{i j}$. From (4),(5), and the uniqueness of $i j$ it follows that $X \cap\left(V(S, N) \backslash S_{i j}\right)=A_{1} \cup A_{2}$. Hence $n \geq 2$, for if $n=1$ then we can add $f_{1}$ to $N_{i}, N_{j}$ and $S_{i j}$, contrary to the maximality of the strip system. Choose $x_{1} \in A_{1}$ and $x_{2} \in A_{2}$ in disjoint strips. From (4), $x_{1}$ is adjacent to exactly one of $f_{1}, f_{n}$, say $f_{1}$. For any other vertex $x_{3} \in A_{2}$, let $R_{u v}(u v \in E(J))$ be a choice of rungs forming $L(H)$ say, such that $x_{1}, x_{3} \in V(H)$. From (4) and (5) it follows that $f_{n}$ is adjacent to $x_{3}$; and so $f_{n}$ is complete to $A_{2}$, and similarly $f_{1}$ is complete to $A_{1}$. From the minimality of $F$, there are no other edges between $F$ and $A_{1} \cup A_{2}$; but then we can add $f_{1}$ to $N_{i}, f_{n}$ to $N_{j}$, and $F$ to $S_{i j}$, contrary to the maximality of the strip system. This proves (6).

Let us say a choice $R_{u v}$ ( $\left.u v \in E(J)\right)$ is optimal if $R_{u v}$ has a vertex in $X$ for all edges $u v$ in $K$. For any choice of rungs, there is an optimal choice with the same traversal (just replace rungs that miss $X$ by rungs that meet $X$ wherever possible); so (6) implies that there are two optimal choices of rungs with different traversals. Now for any optimal choice of rungs, if $h i$ is its traversal, then by (4) and the optimality of the choice, it follows that $K$ consists precisely of the edges of $J$ with exactly one end in common with $h i$, together possibly with $h i$ itself. In particular $h i$ meets all edges in $K$. We may assume that some other edge $j k$ is the traversal for some other optimal choice; and hence (since $J$ is 3 -connected) it follows that $J=K_{4}$ and $j k$ is disjoint from $h i$, and neither edge is in $K$. Hence $V(J)=\{h, i, j, k\}$. Now since the strip system is not degenerate, there is one of the four edges $h j, h k, i j, i k$ whose strip contains a rung of nonzero length; some $h j$-rung $R$ has length $>0$ say. From (4) it follows that exactly one vertex of $R$ is in $X$, one of its ends; say the end in $N_{h}$. Let $R_{u v}(u v \in E(J))$ be any choice of rungs such that $R_{h j}=R$. Since the end of $R$ in $N_{j}$ does not belong to $X$, it follows from (4) that for each of $R_{h k}, R_{i j}, R_{i k}$, its unique vertex in $X$ is its end in $N_{h} \cup N_{i}$. Since the choice of these rungs was arbitrary, it follows that $X \cap S_{h k}=N_{h k}$, $X \cap S_{i j}=N_{i j}$, and $X \cap S_{i k}=N_{i k}$. If also $X \cap S_{h j}=N_{h j}$ then $h i$ is the traversal for every choice of rungs, contrary to (6), so $X \cap S_{h j} \neq N_{h j}$. It follows that every ij-rung has length 0; for if one, $R^{\prime}$ say, has length $>0$, then its unique vertex in $X$ is its end in $N_{i}$, and by exchanging $h$ and $i$ it follows that $X \cap S_{h j}=N_{h j}$, a contradiction. Similarly all $h k$ and $i k$-rungs have length 0 , and therefore all $h j$-rungs have even length, since $G$ is Berge. From (1), we may assume that $f_{1}$ is adjacent to $r_{h j}$ 
and complete to $S_{h k}$, and $f_{n}$ is complete to $S_{i j} \cup S_{i k}$, and there are no other edges between $F$ and $S_{h k} \cup S_{i j} \cup S_{i k} \cup\left\{r_{h j}\right\}$. Let $R^{\prime}$ be an $h j$-rung such that its vertex in $N_{h}$ ( $r_{h j}^{\prime}$, say) is not its unique vertex in $X$. Consequently, its other end $\left(r_{j h}^{\prime}\right)$ is its unique vertex in $X$. By the same argument with $h i$ and $j k$ exchanged, it follows that one of $f_{1}, f_{n}$ is complete to $S_{i j} \cup\left\{r_{j h}^{\prime}\right\}$ and the other to $S_{h k} \cup S_{i k}$; and hence $n=1$. But then the path $f_{1}-r_{h j^{-}}-R_{h j^{-}} r_{j h^{-}} r_{j i^{-}} f_{1}$ is an odd hole, a contradiction. This proves 8.5.

We are now ready to prove 5.4, which we restate:

8.6 Let $G$ be Berge. Let $J$ be a 3-connected graph, such that there is no J-enlargement with a nondegenerate appearance in $G$. Let $L\left(H_{0}\right)$ be an appearance of $J$ in $G$, such that if $L\left(H_{0}\right)$ is degenerate, then $H_{0}=J=K_{3,3}$ and no J-enlargement appears in $\bar{G}$. Then either $G=L\left(H_{0}\right)$, or $H_{0} \neq K_{3,3}$ and $G$ admits a proper 2-join, or $G$ admits a balanced skew partition.

Proof. By 7.5, we may assume that if $J=K_{4}$ or $K_{3,3}$ then no appearance of $J$ in $G$ is overshadowed. Regard $L\left(H_{0}\right)$ as a $J$-strip system in the natural way, and enlarge it to a maximal $J$-strip system $(S, N)$. If $L\left(H_{0}\right)$ is nondegenerate then so is the strip system. Let $Y$ be the set of vertices in $V(G) \backslash V(S, N)$ that are major with respect to the strip system, and let $Z=V(G) \backslash(V(S, N) \cup Y)$. By 8.5, for each component of $Z$, its set of attachments in $V(S, N)$ is local.

\section{(1) If $Y \neq \emptyset$ then $G$ admits a balanced skew partition.}

For suppose not. Let $Y^{\prime}$ be an anticomponent of $Y$, and let $X$ be the set of all $Y^{\prime}$-complete vertices in $V(G)$. For every choice of rungs, forming $L(H)$ say, every member of $Y^{\prime}$ is major with respect to $L(H)$. We claim that $X$ saturates $L(H)$; for suppose not. By 6.1, one of the five outcomes of 6.1 holds. The first we have already assumed is false. Thus 6.1 implies that $L(H)$ is degenerate, and consequently 8.3 implies that $L\left(H_{0}\right)$ is degenerate. By hypothesis, $J=K_{3,3}$, and no $J$-enlargement appears in $\bar{G}$. By 6.1, there is an overshadowed appearance of $J$ in $\bar{G}$, contrary to 7.5 applied in $\bar{G}$. This proves that $X$ saturates $L(H)$. Since this holds for every choice of rungs, it follows that $X$ saturates the strip system. Let $b_{1} b_{2}$ be an edge of $J$, chosen if possible such that $S_{b_{1} b_{2}} \nsubseteq X$. Now the sets $\left(N_{b_{1} v}: b_{1} v \in E(J)\right)$ form a partition of $N_{b_{1}}$ into say $m$ sets, and at least $m-1$ of them are subsets of $X$. Choose $m-1$ of them that are subsets of $X$, not using $N_{b_{1} b_{2}}$ if possible (that is, if the other $m-1$ sets are all subsets of $X$ ), and let their union be $X_{1}$. Define $X_{2} \subseteq N_{b_{2}}$ similarly. We note that $S_{b_{1} b_{2}} \nsubseteq X_{1} \cup X_{2}$; for if some vertex of $S_{b_{1} b_{2}}$ is not in $X$ then this is clear, while if $S_{b_{1} b_{2}} \subseteq X$ then $V(S, N) \subseteq X$ from our choice of $b_{1} b_{2}$, and then from the way we chose $X_{1}$ it follows that $X_{1} \cap S_{b_{1} b_{2}}=\emptyset$, and similarly $X_{2} \cap S_{b_{1} b_{2}}=\emptyset$, and again our claim holds. This proves that $S_{b_{1} b_{2}} \not \subset X_{1} \cup X_{2}$. Define $X_{3}$ to be the set of vertices in $X \cap V(S, N)$ that are not in $X_{1} \cup X_{2}$, and let $X_{0}$ be the set of vertices of $X$ that are not in $V(S, N)$. So $X_{0}, X_{1}, X_{2}, X_{3}$ are four disjoint subsets of $X$, with union $X$. Note that $Y \backslash Y^{\prime} \subseteq X_{0}$. Let $B$ be the union of all components of $G \backslash\left(Y^{\prime} \cup X_{0} \cup X_{1} \cup X_{2}\right)$ that have nonempty intersection with $V(S, N) \backslash S_{b_{1} b_{2}}$, and let $A$ be the union of all the other components. We claim that $B$ is nonempty; for there is an edge $c_{1} c_{2}$ of $J$ disjoint from $b_{1} b_{2}$, and no vertex of $S_{c_{1} c_{2}}$ is in $N_{b_{1}} \cup N_{b_{2}} \cup S_{b_{1} b_{2}}$, and therefore no vertex of $S_{c_{1} c_{2}}$ is in $Y^{\prime} \cup X_{0} \cup X_{1} \cup X_{2}$. Suppose that $A$ is also nonempty. Then $\left(A \cup B, Y^{\prime} \cup X_{0} \cup X_{1} \cup X_{2}\right)$ is a skew partition of $G$. By 4.2 it is not loose; and so $X_{3}$ is empty (since any vertex of $X_{3}$ is in $A \cup B$ and yet is complete to $\left.Y^{\prime}\right)$. In particular, $X \cap V\left(L\left(H_{0}\right)\right) \subseteq N_{b_{1}} \cup N_{b_{2}}$. Since $X \cap V\left(L\left(H_{0}\right)\right)$ saturates $L\left(H_{0}\right)$, it follows that for every vertex $w$ of $J$ different from $b_{1}, b_{2}, w$ has at most one neighbour in 
$J$ different from $b_{1}, b_{2}$, and $w$ is adjacent in $J$ to both $b_{1}$ and $b_{2}$, and all $w b_{1}$ and $w b_{2}$-rungs have length 0 . Since $J$ is 3-connected it follows that $J=K_{4}$, and $L\left(H_{0}\right)$ is degenerate, a contradiction. Thus $A$ is empty. Now we already saw that $S_{b_{1} b_{2}} \nsubseteq X_{1} \cup X_{2}$. Since $A$ is empty, it follows that there is a path of $G$ between $S_{b_{1} b_{2}}$ and $V(S, N) \backslash S_{b_{1} b_{2}}$, disjoint from $Y^{\prime} \cup X_{0} \cup X_{1} \cup X_{2}$. Choose such a path, minimal. From the choice of $X_{1}$ and $X_{2}$ this path has a nonempty interior; from its minimality, none of its internal vertices belong to $V(S, N)$; since all major vertices are in $Y^{\prime} \cup X_{0}$, its interior contains no major vertices; by 8.5, the set of attachments of its interior is local; yet its ends are both attachments of its interior, so there exist $u \in S_{b_{1} b_{2}}$ and $v \in V(S, N) \backslash S_{b_{1} b_{2}}$, such that $u, v \notin X_{1} \cup X_{2}$, and yet $\{u, v\}$ is local. Now $u, v$ do not lie in the same strip, and therefore there is some $N_{w}$ containing them both; and the only $w \in V(J)$ with $u \in N_{w}$ are $b_{1}, b_{2}$, so we may assume that $u, v \in N_{b_{1}}$. Since they are not in $X_{1}$, and not in the same strip, this is impossible. This proves (1).

We may therefore assume that $Y$ is empty.

(2) If there is a component $F$ of $Z$ such that for some $v \in V(J)$, all attachments of $F$ in $V(S, N)$ belong to $N_{v}$, then $G$ admits a balanced skew partition.

For let $F^{\prime}=V(G) \backslash\left(F \cup N_{v}\right)$; then $F^{\prime} \neq \emptyset$, and every path in $G$ from $F$ to $F^{\prime}$ meets $N_{v}$. Since $N_{v}$ is not anticonnected, it follows that $\left(F \cup F^{\prime}, N_{v}\right)$ is a skew partition. By 4.2 we may assume it is not loose, and we will prove that it is balanced. Let the neighbours of $v$ in $J$ be $u_{1}, \ldots, u_{k}$; then every anticomponent of $N_{v}$ is a subset of one of $N_{v u_{1}}, \ldots, N_{v u_{k}}$. Choose a neighbour $w$ of $u_{1}$ in $J$ different from $v, u_{2}$, choose $n_{1} \in N_{u_{1} w}$, and choose $n_{2} \in N_{v u_{2}}$. Then $n_{1}, n_{2}$ belong to strips $S_{u_{1} w}, S_{v u_{2}}$, where $u_{1} w, v u_{2}$ are disjoint edges of $J$; and so $n_{1}, n_{2}$ are not adjacent in $G$. Let $K=\left\{n_{1}\right\} \cup S_{v u_{1}} \backslash N_{v u_{1}}$. Then $K$ is connected (since every vertex of $S_{v u_{1}}$ is in a $v u_{1}$-rung and $n_{1}$ is complete to $N_{u_{1} v}$ ), every vertex in $N_{v u_{1}}$ has a neighbour in $K$ (for the same reason), and $n_{2}$ is not in $K$ and has no neighbour in $K$. (For the last claim, $n_{2}$ is not in $K$ since it is in only one strip; and it has no neighbour in $S_{v u_{1}} \backslash N_{v u_{1}}$ from the definition of a strip system; and it is not adjacent to $n_{1}$ as we already saw.) By 2.6, $\left(K, N_{v u_{1}}\right)$ is balanced, and therefore by 2.7 .1 , so is $\left(F, N_{v u_{1}}\right)$. By $4.5, G$ admits a balanced skew partition. This proves (2).

We assume therefore that there are no such components $F$ of $Z$. Consequently, for every component $F$ of $Z$, there is an edge $b_{1} b_{2}$ of $J$ such that all the attachments of $F$ in $V(S, N)$ are in $S_{b_{1} b_{2}}$. If $Z$ is empty and for all $b_{1} b_{2}$ there is only one $b_{1} b_{2}$-rung, then $G=L\left(H_{0}\right)$ and the theorem holds. So we may assume that there is an edge $b_{1} b_{2}$ of $J$ such that either there is more than one $b_{1} b_{2}$-rung in $S_{b_{1} b_{2}}$ or there is a component $F$ of $Z$ with all its attachments in $S_{b_{1} b_{2}}$. Let $A$ be the union of $S_{b_{1} b_{2}}$ and any components of $Z$ that have an attachment in $S_{b_{1} b_{2}}$ (and which therefore have attachments only in $S_{b_{1} b_{2}}$ ), and let $B=V(G) \backslash A$. Let $A_{1}=N_{b_{1} b_{2}}, A_{2}=N_{b_{2} b_{1}}, B_{1}=N_{b_{1}} \backslash N_{b_{1} b_{2}}$, and $B_{2}=N_{b_{2}} \backslash N_{b_{2} b_{1}}$. Then $A_{1}, A_{2} \subseteq A$, and $B_{1}, B_{2}$ are disjoint subsets of $B$, and for $i=1,2 A_{i}$ is complete to $B_{i}$, and there are no other edges between $A$ and $B$. Also $\left|B_{1}\right| \geq 2$, and we chose $b_{1} b_{2}$ such that if $A_{1}, A_{2}$ both have only one vertex then $A$ is not the vertex set of a path joining them. If $A_{1} \cap A_{2}=\emptyset$ then $H_{0} \neq K_{3,3}$ and $G$ admits a proper 2-join, and the theorem holds. Thus we may assume that there exists $a \in A_{1} \cap A_{2} \neq \emptyset$. Then $a$ is complete to $B_{1} \cup B_{2}$, and since $|A| \geq 2$, it follows that $\left(\left(B \backslash\left(B_{1} \cup B_{2}\right)\right) \cup(A \backslash\{a\}), B_{1} \cup B_{2} \cup\{a\}\right)$ is a skew partition of $G$. Since $\{a\}$ is an anticomponent of $B_{1} \cup B_{2} \cup\{a\}, 4.1$ implies that $G$ admits a balanced skew partition. This proves 


\section{$9 \quad$ Double split graphs}

In this section we handle degenerate appearances of $K_{4}$. There is another way to view them, not as line graphs but as sets of paths and antipaths with certain properties, as we shall see.

Let $P_{1}, P_{2}$ be paths in a graph $G$, and let $Q_{1}, Q_{2}$ be antipaths. Suppose that $P_{1}, P_{2}, Q_{1}, Q_{2}$ are pairwise disjoint, and we can label the ends of each $P_{i}$ as $a_{i}, b_{i}$, and label the ends of each $Q_{j}$ as $x_{j}, y_{j}$, such that:

- $P_{1}, P_{2}, Q_{1}, Q_{2}$ all have length $\geq 1$

- there are no edges between $P_{1}$ and $P_{2}$, and $Q_{1}$ is complete to $Q_{2}$

- for $(i, j)=(1,1),(1,2)$ or $(2,1)$, the only edges between $V\left(P_{i}\right)$ and $\left\{x_{j}, y_{j}\right\}$ are $a_{i} x_{j}$ and $b_{i} y_{j}$, and the only edges between $V\left(P_{2}\right)$ and $\left\{x_{2}, y_{2}\right\}$ are $a_{2} y_{2}$ and $b_{2} x_{2}$,

- for $(i, j)=(1,1),(1,2)$ or $(2,1)$, the only nonedges between $V\left(Q_{j}\right)$ and $\left\{a_{i}, b_{i}\right\}$ are $a_{i} y_{j}$ and $b_{i} x_{j}$, and the only nonedges between $V\left(Q_{2}\right)$ and $\left\{a_{2}, b_{2}\right\}$ are $a_{2} x_{2}$ and $b_{2} y_{2}$.

In these circumstances we call the quadruple $\left(P_{1}, P_{2}, Q_{1}, Q_{2}\right)$ a knot in $G$. Note that if $\left(P_{1}, P_{2}, Q_{1}, Q_{2}\right)$ is a knot then so is $\left(P_{2}, P_{1}, Q_{1}, Q_{2}\right)$, with a suitable relabelling of the ends of the paths and antipaths.

If $L(H)$ is a degenerate appearance of $K_{4}$ in $G$, it can be viewed as a knot. For, in our usual notation, let $R_{1,3}, R_{1,4}, R_{2,3}, R_{2,4}$ have length 0 ; let $P_{1}=R_{1,2}, P_{2}=R_{3,4}$, let $Q_{1}$ be the antipath $r_{1,3}-r_{2,4}$, and $Q_{2}$ the antipath $r_{1,4}-r_{2,3}$. It is easy to check that this is a knot. In fact, this and its complement are the only knots in Berge graphs, as the next theorem shows.

9.1 Let $\left(P_{1}, P_{2}, Q_{1}, Q_{2}\right)$ be a knot in a Berge graph $G$. Then all four of $P_{1}, P_{2}, Q_{1}, Q_{2}$ have odd length; and either both $P_{1}, P_{2}$ have length 1 , or both $Q_{1}, Q_{2}$ have length 1 .

Proof. Define $a_{i}, b_{i}, x_{i}, y_{i}(i=1,2)$ as usual. Certainly $P_{1}$ is odd since $x_{1}-a_{1}-P_{1}-b_{1}-y_{2}-x_{1}$ is a hole, and similarly the other three are odd. Suppose one of $P_{1}, P_{2}$ has length $>1$ and one of $Q_{1}, Q_{2}$ has length $>1$. By exchanging $P_{1}, P_{2}$ or $Q_{1}, Q_{2}$ we may therefore assume that $P_{1}, Q_{1}$ both have length $>1$. Let $Y$ be the interior of $Q_{1}$. Then $a_{1}, b_{1}, a_{2}, b_{2}$ are all $Y$-complete, from the last condition in the definition of a knot, and since $a_{2}$ has no neighbours in the interior of $P_{1}$ it follows from 2.2 that there is a $Y$-complete vertex ( $v$ say) in the interior of $P_{1}$. But $x_{1}, y_{1}$ are not $Y$-complete, and they are adjacent, so $a_{1}-x_{1}-y_{1}-b_{1}$ is an odd path between $Y$-complete vertices and $v$ has no neighbour in its interior, contrary to 2.2. This proves 9.1.

Nevertheless, it turns out to be advantageous to make only limited use of 9.1 ; it is better to preserve the symmetry between the paths and the antipaths.

Let $\left(P_{1}, P_{2}, Q_{1}, Q_{2}\right)$ be a knot in a Berge graph $G$; we define $K$ to be the subgraph of $G$ induced on $V\left(P_{1}\right) \cup V\left(P_{2}\right) \cup V\left(Q_{1}\right) \cup V\left(Q_{2}\right)$. (For brevity we say that the knot induces $K$.) We say a subset $X \subseteq V(K)$ is local (with respect to the knot) if $X$ is disjoint from one of $V\left(P_{1}\right), V\left(P_{2}\right)$, and $X$ includes neither of $V\left(Q_{1}\right), V\left(Q_{2}\right)$, and $X \cap\left(V\left(P_{1}\right) \cup V\left(P_{2}\right)\right)$ is complete to $X \cap\left(V\left(Q_{1}\right) \cup V\left(Q_{2}\right)\right)$. We say $X$ resolves the knot if $V(K) \backslash X$ is local with respect to the knot $\left(Q_{1}, Q_{2}, P_{1}, P_{2}\right)$ in $\bar{G}$; that is, if 
$X$ includes one of $V\left(Q_{1}\right), V\left(Q_{2}\right)$, and $X$ meets both $P_{1}$ and $P_{2}$, and $X$ contains at least one end of every edge between $V\left(P_{1}\right) \cup V\left(P_{2}\right)$ and $V\left(Q_{1}\right) \cup V\left(Q_{2}\right)$. Conveniently, these definitions almost agree with what we did for line graphs, because of the following.

9.2 Let $\left(P_{1}, P_{2}, Q_{1}, Q_{2}\right)$ be a knot in a graph $G$, inducing $K$, where $Q_{1}, Q_{2}$ both have length 1 , and so $K=L(H)$ is an appearance of $K_{4}$. Let $X \subseteq V(K)$. Then:

- $X$ is local with respect to the knot if and only if it is local with respect to $L(H)$

- $X$ resolves the knot if and only if $X$ saturates $L(H)$ and $X$ meets both $V\left(P_{1}\right)$ and $V\left(P_{2}\right)$.

The proof is obvious and we omit it. This allows us to unify some portions of 5.8 and 6.1 , as follows. (The expression "up to symmetry" means here "possibly after exchanging $P_{1}$ and $P_{2}$ and exchanging $Q_{1}$ and $Q_{2}$, and renaming the ends of $P_{1}, P_{2}, Q_{1}, Q_{2}$ accordingly.")

9.3 Let $\left(P_{1}, P_{2}, Q_{1}, Q_{2}\right)$ be a knot in a Berge graph $G$, inducing $K$. Assume that there is no appearance in $G$ or in $\bar{G}$ of any $K_{4}$-enlargement, and there is no overshadowed appearance of $K_{4}$ in $G$ or in $\bar{G}$. Let $F$ be a connected subset of $V(G) \backslash V(K)$, such that its set of attachments in $K$ is not local. Then either:

1. there is a vertex in $F$ such that its neighbour set in $K$ resolves the knot, or

2. (up to symmetry) there is a path $R$ in $F$ with ends $r_{1}, r_{2}$ such that $r_{1}, a_{1}$ have the same neighbours in $V\left(P_{2}\right) \cup V\left(Q_{1}\right) \cup V\left(Q_{2}\right)$, and there are no edges between $R \backslash r_{1}$ and $V\left(P_{2}\right) \cup V\left(Q_{1}\right) \cup$ $V\left(Q_{2}\right)$, and $r_{2}$ has a neighbour in $P_{1} \backslash a_{1}$, and there are no edges between $R \backslash r_{2}$ and $P_{1} \backslash a_{1}$, or

3. (up to symmetry) there is an odd path $R$ in $F$ with ends $r_{1}, r_{2}$ such that $r_{1}, a_{1}$ have the same neighbours in $V\left(P_{2}\right) \cup V\left(Q_{1}\right) \cup V\left(Q_{2}\right)$, and $r_{2}, b_{1}$ have the same neighbours in $V\left(P_{2}\right) \cup V\left(Q_{1}\right) \cup$ $V\left(Q_{2}\right)$, and there are no edges between $V\left(R^{*}\right)$ and $V\left(P_{2}\right) \cup V\left(Q_{1}\right) \cup V\left(Q_{2}\right)$, and no edges between $R$ and $P_{1}$ except possibly $r_{1} a_{1}$ and $r_{2} b_{1}$, or

4. there is a vertex $f \in F$ such that (up to symmetry) $f, x_{1}$ have the same neighbours in $V\left(P_{1}\right) \cup$ $V\left(P_{2}\right) \cup V\left(Q_{2}\right)$ and $f$ is not adjacent to $y_{1}$.

Proof. Define $a_{i}, b_{i}, x_{i}, y_{i}(i=1,2)$ as usual. By 9.1 there are two cases, depending whether $Q_{1}$ and $Q_{2}$ have length 1 or $P_{1}, P_{2}$ have length 1 .

(1) If $Q_{1}, Q_{2}$ have length 1 then the theorem holds.

For assume $Q_{1}, Q_{2}$ have length 1 . Then $K$ is a degenerate appearance of $K_{4}$ in $G$, say $K=L(H)$. Suppose that the neighbour set of some $f \in F$ saturates $L(H)$. If $f$ has a neighbour in both $V\left(P_{1}\right)$ and $V\left(P_{2}\right)$ then statement 1 of the theorem holds, so we assume it has no neighbour in $V\left(P_{1}\right)$. But then $f$ is adjacent to all four of $x_{1}, x_{2}, y_{1}, y_{2}$, since it has two neighbours in every triangle of $K$, and then $f-x_{1}-a_{1}-P_{1}-b_{1}-y_{1}-f$ is an odd hole, a contradiction. So we assume there is no such $f$, and hence we may apply 5.8. If 5.8.1 holds then there is an appearance in $G$ of some $K_{4}$-enlargement, a contradiction. So 5.8.2 holds. In the notation of 5.8.2, the edge $b_{1} b_{2}$ of $J$ is of one of two types; either $N_{b_{1}}$ meets $N_{b_{2}}$ or it does not. In the first case, we may assume from the symmetry that those 
two sets are $\left\{x_{1}, x_{2}, a_{1}\right\}$ and $\left\{x_{1}, y_{2}, a_{2}\right\}$, and there is a path $R$ of $G$ with $V(R) \subseteq F$ and with ends $r_{1}$ and $r_{2}$, such that $r_{1}$ is adjacent to $a_{1}, x_{2}$, and $r_{2}$ is adjacent to $a_{2}, y_{2}$, and there are no other edges between $V(P)$ and $K \backslash x_{1}$. If $R$ has length 0 then statement 4 of the theorem holds, while if $R$ has length $>0$ then it is even and there is an overshadowed appearance of $K_{4}$ in $G$, a contradiction. In the second case, when the sets called $N\left(b_{1}\right), N\left(b_{2}\right)$ in the notation of 5.8 .2 are disjoint, we may assume that these sets are $\left\{x_{1}, x_{2}, a_{1}\right\}$ and $\left\{y_{1}, y_{2}, b_{1}\right\}$ respectively, and one of 5.8.2(a), 5.8.2(b), 5.8.2(c) holds. In the first two cases statements 2,3 of the theorem hold, respectively, and the last case is impossible since $P_{1}$ is odd. This proves (1).

Henceforth we may therefore assume that one of $Q_{1}, Q_{2}$ has length $>1$, and therefore by 9.1 , both $P_{1}$ and $P_{2}$ have length 1 . Hence $\bar{K}=L(H)$, where $L(H)$ is a degenerate appearance of $K_{4}$ in $\bar{G}$.

(2) If there exists $f \in F$ such that $f$ is not major with respect to $L(H)$ in $\bar{G}$, then the theorem holds.

For let $f \in F$ have this property. If the set of neighbours of $f$ in $K$ resolves the knot $\left(P_{1}, P_{2}, Q_{1}, Q_{2}\right)$, then statement 1 of the theorem holds, so we assume not. Therefore, in $\bar{G}$, the set of neighbours of $f$ in $\bar{K}$ is not local with respect to the knot $\left(\overline{Q_{1}}, \overline{Q_{2}}, \overline{P_{1}}, \overline{P_{2}}\right)$. But this set does not saturate $L(H)$; so we can apply 5.8 (or, indeed, 5.7) in $\bar{G}$, and deduce, as before, that either there is a $K_{4}$-enlargement that appears in $\bar{G}$ (a contradiction), or (up to symmetry) $f, a_{1}$ have the same neighbours in $K \backslash a_{1}$ (but then statement 2 of the theorem holds), or (up to symmetry) $f, x_{1}$ have the same neighbours in $V\left(P_{1}\right) \cup V\left(P_{2}\right) \cup V\left(Q_{2}\right)$ (but then either statement 1 or statement 4 of the theorem holds). This proves (2).

We may therefore assume that every $f \in F$ is major with respect to $L(H)$ in $\bar{G}$. Let $X$ be the set of vertices of $K$ which, in $G$, have no neighbours in $F$. By hypothesis, $V(K) \backslash X$ is not local with respect to the knot $\left(P_{1}, P_{2}, Q_{1}, Q_{2}\right)$ in $G$, and hence $X$ does not resolve the knot $\left(\overline{Q_{1}}, \overline{Q_{2}}, \overline{P_{1}}, \overline{P_{2}}\right)$ in $\bar{G}$. If $X$ does not saturate $L(H)$ in $\bar{G}$, then by (2) we may apply 6.1 . Since $Q_{1}$ has length $>1$ it follows that the last outcome of 6.1 holds, and hence statement 3 of the theorem holds. We may therefore assume that $X$ saturates $L(H)$ in $\bar{G}$. By 9.2, $X$ is disjoint from one of $V\left(Q_{1}\right), V\left(Q_{2}\right)$, say $X \cap V\left(Q_{1}\right)=\emptyset$. Hence $a_{1}, a_{2}, b_{1}, b_{2} \in X$. Since $a_{1}-y_{1}-Q_{1}-x_{1}-b_{1}$ is an odd antipath in $G$, and its internal vertices all have neighbours in $F$, and its ends do not, it follows from 2.2 applied in $\bar{G}$ that every vertex in $X$ has a non-neighbour in $V\left(Q_{1}\right)$; and hence no vertex of $Q_{2}$ belongs to $X$. This restores the symmetry between $Q_{1}, Q_{2}$. Now one of $Q_{1}, Q_{2}$ has length $>1$, say $Q_{1}$ without loss of generality. Hence, in $\bar{G}$, the path $a_{1}-y_{1}-Q_{1}-x_{1}-b_{1}$ is odd and has length $\geq 5$; its ends are complete to $F$, and its internal vertices are not. By $2.1, F$ contains a leap; so there exist nonadjacent $f_{1}, f_{2} \in F$ such that $Q_{1}$ is the interior of a path $R$ between them. (All this is in $\bar{G}$ - we will tell the reader when we switch back to $G$.) Now $f_{1}, f_{2}$ have no common neighbour in $Q_{2}$ (because $R$ could be completed to an odd hole through any such common neighbour), so by $2.1, f_{1}, f_{2}$ is also a leap for the path $a_{1}-y_{2}-Q_{2}-x_{2}-b_{1}$ (this path might have length 3 , but still we get a leap by 2.1 .3 , since $\left\{f_{1}, f_{2}\right\}$ cannot include the interior of any longer antipath between $x_{2}$ and $y_{2}$ ). Hence from the symmetry we may assume that $f_{1}$ is adjacent to $y_{1}, y_{2}$, and $f_{2}$ to $x_{1}, x_{2}$, and there are no other edges between $\left\{f_{1}, f_{2}\right\}$ and $V\left(Q_{1}\right) \cup V\left(Q_{2}\right)$. Therefore, back in $G$, we see that $a_{1}, f_{1}$ have the same neighbours in $V\left(P_{2}\right) \cup V\left(Q_{1}\right) \cup V\left(Q_{2}\right)$, and so do $b_{1}, f_{2}$, and therefore statement 3 of the theorem holds. This proves 9.3 . 
9.3 suggests that we should attempt to combine paths into strips, as in the section on "Generalized line graphs", and combine antipaths into "antistrips". Let us make that precise.

Let $A, B, C$ be disjoint subsets of $V(G)$. We call $S=(A, C, B)$ a strip if $A, B$ are nonempty, and every vertex of $A \cup B \cup C$ belongs to a path between $A$ and $B$ with only its first vertex in $A$, only its last vertex in $B$, and interior in $C$. Such a path is called a rung of the strip $S$, or an $S$-rung. When $S=(A, C, B)$ is a strip, $V(S)$ means $A \cup B \cup C$. The reverse of a strip $(A, C, B)$ is the strip $(B, C, A)$. An antistrip is a triple that is a strip in $\bar{G}$, and the corresponding antipaths are called antirungs. If $P$ is a rung with ends $a \in A$ and $b \in B$, we speak of the "rung $a-P-b$ " for brevity; the reader can deduce which end is in which set from the names of the ends, because we shall always use $a, a^{\prime}, a_{1}$ etc. for ends in a set called something like $A$, and so on.

Let $S=(A, C, B)$ be a strip and $T=(X, Z, Y)$ an antistrip, with $V(S) \cap V(T)=\emptyset$. We say $S, T$ are parallel if:

- $A$ is complete to $X \cup Z$, and $B$ is complete to $Y \cup Z$, and

- $X$ is anticomplete to $B \cup C$, and $Y$ is anticomplete to $A \cup C$.

We say $S, T$ are co-parallel if $S, T^{\prime}$ are parallel, where $T^{\prime}$ is the reverse of $T$.

Now let $S_{1}, S_{2}$ be strips and $T$ an antistrip, where $S_{1}, S_{2}, T$ are pairwise disjoint. We say that $S_{1}, S_{2}$ agree on $T$ if either $S_{1}, T$ are parallel and $S_{2}, T$ are parallel, or both pairs are co-parallel; and they disagree if one pair is parallel and the other pair is co-parallel. If $S$ is a strip and $T_{1}, T_{2}$ are antistrips, pairwise disjoint, we define whether $T_{1}, T_{2}$ agree or disagree on $S$ similarly.

Now let $S_{1}, S_{2}$ be strips, and let $T_{1}, T_{2}$ be antistrips, all pairwise disjoint. We call the quadruple $\left(S_{1}, S_{2}, T_{1}, T_{2}\right)$ a twist if $S_{1}, S_{2}$ agree on one of $T_{1}, T_{2}$ and disagree on the other. (Equivalently, if $T_{1}, T_{2}$ agree on one of $S_{1}, S_{2}$, and disagree on the other.) Note that if $\left(S_{1}, S_{2}, T_{1}, T_{2}\right)$ is a twist, then so is $\left(S_{1}^{\prime}, S_{2}, T_{1}, T_{2}\right)$, where $S_{1}^{\prime}$ is the reverse of $S_{1}$.

A striation in a graph $G$ is a family of strips $S_{i}=\left(A_{i}, C_{i}, B_{i}\right)(1 \leq i \leq m)$ together with a family of antistrips $T_{j}=\left(X_{j}, Z_{j}, Y_{j}\right)(1 \leq j \leq n)$, satisfying the following conditions:

- all the strips and antistrips are pairwise disjoint, and all their rungs and antirungs have odd length

- $m, n \geq 2$

- for $1 \leq i<i^{\prime} \leq m, S_{i}$ is anticomplete to $S_{i^{\prime}}$, and for $1 \leq j<j^{\prime} \leq n, T_{j}$ is complete to $T_{j^{\prime}}$

- for $1 \leq i \leq m$ and $1 \leq j \leq n, S_{i}$ and $T_{j}$ are either parallel or co-parallel

- for $1 \leq i<i^{\prime} \leq m$ there exist distinct $j, j^{\prime}$ with $1 \leq j, j^{\prime} \leq n$ such that $\left(S_{i}, S_{i^{\prime}}, T_{j}, T_{j^{\prime}}\right)$ is a twist

- for $1 \leq j<j^{\prime} \leq n$ there exist distinct $i, i^{\prime}$ with $1 \leq i, i^{\prime} \leq m$ such that $\left(S_{i}, S_{i^{\prime}}, T_{j}, T_{j^{\prime}}\right)$ is a twist.

(Note that if we replace some $\left(A_{i}, C_{i}, B_{i}\right)$ by its reverse, we obtain another striation.) We denote the striation by $L$, and the union of the vertex sets of all its strips and antistrips by $V(L)$. By analogy with what we did for knots, let us say that a subset $X \subseteq V(L)$ is local with respect to $L$ if

- at most one of $X \cap V\left(S_{1}\right), \ldots, X \cap V\left(S_{m}\right)$ is nonempty, 
- for $1 \leq j \leq n$, every $T_{j}$-antirung has a vertex not in $X$, and

- $X \cap\left(V\left(S_{1}\right) \cup \cdots \cup V\left(S_{m}\right)\right)$ is complete to $X \cap\left(V\left(T_{1}\right) \cup \cdots \cup V\left(T_{n}\right)\right)$.

We say $X$ resolves $L$ if $V(L) \backslash X$ is local with respect to the striation in $\bar{G}$ obtained from $L$ by exchanging the strips and antistrips; that is, if

- there is at most one of $T_{1}, \ldots, T_{n}$ that is not a subset of $X$,

- for $1 \leq i \leq m$, every $S_{i}$-rung meets $X$, and

- $X$ contains at least one end of every edge between $V\left(S_{1}\right) \cup \cdots \cup V\left(S_{m}\right)$ and $V\left(T_{1}\right) \cup \cdots \cup V\left(T_{n}\right)$.

A striation $L$ in $G$ is maximal if there is no striation $L^{\prime}$ in $G$ with $V(L) \subset V\left(L^{\prime}\right)$.

9.4 Let $G$ be Berge, such that there is no appearance in $G$ or in $\bar{G}$ of any $K_{4}$-enlargement, and there is no overshadowed appearance of $K_{4}$ in $G$ or in $\bar{G}$. Let $L$ be a maximal striation in $G$. Let $f \in V(G) \backslash V(L)$, and let $X$ be the set of neighbours of $f$ in $V(L)$. Then either $X$ is local with respect to $L$, or $X$ resolves $L$.

Proof. Let $L$ have strips $S_{i}=\left(A_{i}, C_{i}, B_{i}\right)(1 \leq i \leq m)$ and antistrips $T_{j}=\left(X_{j}, Z_{j}, Y_{j}\right)(1 \leq j \leq n)$.

(1) Let $1 \leq i \leq m$, and $1 \leq j \leq n$; let $a_{i}-P_{i}-b_{i}$ be an $S_{i}$-rung, and $x_{j}-Q_{j}-y_{j}$ a $T_{j}$-antirung. Then either $X \cap V\left(P_{i}\right) \neq \emptyset$, or $V\left(Q_{j}\right) \nsubseteq X$.

For suppose that $X$ includes $V\left(Q_{1}\right)$ and is disjoint from $V\left(P_{1}\right)$ say. By reversing $S_{2}$ we may assume that $S_{1}$ and $S_{2}$ agree on $T_{1}$; and we may assume they disagree on $T_{2}$. Let $a_{2}-P_{2}$ - $b_{2}$ be any $S_{2}$-rung, and $x_{2}-Q_{2}-y_{2}$ any $T_{2}$-antirung. Then $\left(P_{1}, P_{2}, Q_{1}, Q_{2}\right)$ is a knot, so by 9.1 , we may assume (taking complements if necessary) that $Q_{1}$ has length 1 . But then $f-x_{1}-a_{1}-P_{1}-b_{1}-y_{1}-f$ is an odd hole, a contradiction. This proves (1).

From (1), taking complements if necessary, we may assume that for all $1 \leq j \leq n$, and for all $T_{j}$-antirungs $Q_{j}, V\left(Q_{j}\right) \nsubseteq X$.

(2) $X$ meets at most one of $V\left(S_{1}\right), \ldots, V\left(S_{m}\right)$.

For suppose that $X$ meets both $S_{1}$ and $S_{2}$ say. We may assume that $\left(S_{1}, S_{2}, T_{1}, T_{2}\right)$ is a twist. For $i=1,2$ choose an $S_{i}$-rung $P_{i}$ such that $X \cap V\left(P_{i}\right) \neq \emptyset$, and for $j=1,2$ choose any $T_{j}$-antirung $Q_{j}$. By our assumption above, $f$ has nonneighbours in both $Q_{1}, Q_{2}$. But then $\left(P_{1}, P_{2}, Q_{1}, Q_{2}\right)$ is a knot, and setting $F=\{f\}$ violates 9.3 , a contradiction. This proves (2).

We may assume that $X$ is not local with respect to $L$, and so we may assume that there is an $S_{1}$-rung $a_{1}-P_{1}-b_{1}$ and a $T_{1}$-antirung $x_{1}-Q_{1}-y_{1}$ containing nonadjacent members of $X$. By reversing each $T_{j}$ if necessary, we may assume that $S_{1}$ is parallel to each $T_{j}$. In particular, $a_{1} x_{1}$ is an edge, and so is $b_{1} y_{1}$. Since one of $P_{1}, Q_{1}$ has length 1 by 9.1 , the interior of $Q_{1}$ is complete to $V\left(P_{1}\right)$, we may assume that $x_{1} \in X$, and $X \cap V\left(P_{1} \backslash a_{1}\right) \neq \emptyset$. Let $2 \leq j \leq n$, and let $x_{j}-Q_{j}-y_{j}$ be any $T_{j}$-antirung. For definiteness we assume $j=2$. Now $T_{1}, T_{2}$ agree on $S_{1}$, and so there is some $S_{i}$ on which they 
disagree, say $S_{2}$. Let $a_{2}-P_{2}-b_{2}$ be any $S_{2}$-rung. Then $\left(P_{1}, P_{2}, Q_{1}, Q_{2}\right)$ is a knot, with union $K$ say, and $X \cap V(K)$ is not local with respect to $K$ (since $x_{1} \in X$, and $X \cap V\left(P_{1} \backslash a_{1}\right) \neq \emptyset$ ). By 9.3, it follows that 9.3.2 holds, and hence $f, a_{1}$ have the same neighbours in $V\left(Q_{1}\right) \cup V\left(Q_{2}\right)$. In particular, $V\left(Q_{2}\right) \backslash\left\{y_{2}\right\} \subseteq X$. Since $V\left(Q_{2}\right) \nsubseteq X$, it follows that $y_{2} \notin X$; since this holds for all $Q_{2}$, we deduce that $X \cap V\left(T_{2}\right)=X_{2} \cup Z_{2}$; and since the same holds for all antistrips of $L$ except $T_{1}$, we deduce that $X \cap V\left(T_{j}\right)=X_{j} \cup Z_{j}$ for $2 \leq j \leq n$. Since our only assumption about $T_{1}$ was that $X \cap X_{1} \neq \emptyset$, and since we have shown that the same is true for all $T_{j}$, we can replace $T_{1}$ by $T_{2}$ say, and deduce similarly that $X \cap V\left(T_{1}\right)=X_{1} \cup Z_{1}$. But then we can add $f$ to $A_{1}$, contrary to the maximality of the striation. This proves 9.4.

9.5 Let $G$ be Berge, such that there is no appearance in $G$ or in $\bar{G}$ of any $K_{4}$-enlargement, and there is no overshadowed appearance of $K_{4}$ in $G$ or in $\bar{G}$. Let $L$ be a maximal striation in $G$. Let $F \subseteq V(G) \backslash V(L)$ be connected, such that for each $f \in F$, the set of its neighbours in $V(L)$ is local with respect to $L$. Then the set of attachments of $F$ in $V(L)$ is local with respect to $L$.

Proof. Let $L$ have strips $S_{i}=\left(A_{i}, C_{i}, B_{i}\right)(1 \leq i \leq m)$ and antistrips $T_{j}=\left(X_{j}, Z_{j}, Y_{j}\right)(1 \leq j \leq n)$. Suppose not, and choose a counterexample $F$ with $F$ minimal. Let $X$ be its set of attachments in $V(L)$.

(1) $X \nsubseteq V\left(T_{1}\right) \cup-\cdots-\cdots V\left(T_{n}\right)$.

For suppose it is. Since $X$ is not local, we may assume that $X$ includes $V\left(Q_{1}\right)$ for some $T_{1}$-antirung $x_{1}-Q_{1}-y_{1}$. Let $2 \leq j \leq n$, and let $x_{j}-Q_{j}-y_{j}$ be a $T_{j}$-antirung. Then we can choose some $S_{i}, S_{i^{\prime}}$ to make a twist, and if we choose an $S_{i}$-rung and $S_{i^{\prime}}$-rung and apply 9.3 to the resultant knot, we deduce (since no vertices of $S_{i}$ and $S_{i^{\prime}}$ are in $X$ ) that 9.3.3 holds. This has several consequences. First, it implies that there is an odd path in $F$ with vertices $f_{1}, \ldots, f_{k}$ say, which is either parallel or coparallel to $Q_{1}$, and either parallel or co-parallel to $Q_{j}$; and there are no edges between $\left\{f_{2}, \ldots, f_{k-1}\right\}$ and $Q_{1} \cup Q_{j}$. Hence the set of attachments of $\left\{f_{1}, \ldots, f_{k}\right\}$ is not local with respect to $L$, and so $F=\left\{f_{1}, \ldots, f_{k}\right\}$ from the minimality of $F$. Second, every vertex of $Q_{j}$ is in $X$, and since this holds for all $Q_{j}$ it follows that $V\left(T_{j}\right) \subseteq X$. By exchanging $T_{1}$ and $T_{j}$ it follows that $V\left(T_{1}\right) \subseteq X$. Moreover, since this holds for all $j$ we deduce that $X=V\left(T_{1}\right) \cup \cdots \cup V\left(T_{n}\right)$. This restores the symmetry between $T_{1}$ and $T_{2}, \ldots, T_{n}$. Third, this shows that there are no edges between $\left\{f_{2}, \ldots, f_{k-1}\right\}$ and $V\left(T_{1}\right) \cup \cdots \cup V\left(T_{n}\right)$. Fourth, for $1 \leq j \leq n$ every vertex in $Z_{j}$ is adjacent to both $f_{1}, f_{k}$. Since $k$ is even, this proves that either $k=2$ or $Z_{1} \cup \cdots \cup Z_{n}=\emptyset$. Fifth, every vertex in $X_{1} \cup Y_{1} \cdots \cup X_{n} \cup Y_{n}$ is adjacent to exactly one of $f_{1}, f_{n}$; let $U$ be the set of those adjacent to $f_{1}$, and $V$ those adjacent to $f_{n}$. For the moment fix $j$ with $1 \leq j \leq n$. Every $T_{j}$-antirung has one end in $U$ and the other in $V$; let $M_{j}$ be the union of the vertex sets of all $T_{j}$-antirungs $x_{j}-Q_{j}-y_{j}$ such that $x_{j} \in U$, and $N_{j}$ the union of all those with $x_{j} \in V$. Since there is no $T_{j}$-antirung with both ends in $M_{j}$ or both ends in $N_{j}$, it follows that $M_{j} \cap N_{j}=\emptyset$, and there are no nonedges betwen $M_{j}$ and $N_{j}$ except possibly between $M_{j} \cap X_{j}$ and $N_{j} \cap X_{j}$, or between $M_{j} \cap Y_{j}$ and $N_{j} \cap Y_{j}$. Suppose there is such a nonedge; and choose $T_{j}$-antirungs $x_{j}-Q_{j}-y_{j}, x_{j}^{\prime}-Q_{j}^{\prime}-y_{j}^{\prime}$ where $x_{j} \in U$ is nonadjacent to $x_{j}^{\prime} \in V$, say. Now $x_{j}, x_{j}^{\prime}$ have a common neighbour $d_{1} \in A_{1} \cup B_{1}$, and then $d_{1}-x_{j^{-}} f_{1^{-}} \cdots-f_{k^{-}} x_{j}^{\prime}-d_{1}$ is an odd hole. This proves that $M_{j}$ is complete to $N_{j}$. Now if $M_{j}$ is nonempty, then $\left(M_{j} \cap X_{j}, M_{j} \cap Z_{j}, M_{j} \cap Y_{j}\right)$ is an antistrip, and similarly if $N_{j}$ is nonempty it also induces an antistrip. We call these the offspring of $T_{j}$. (If 
one of $M_{j}, N_{j}$ is empty, then the other equals $V\left(T_{j}\right)$, and so the only offspring of $T_{j}$ is $T_{j}$ itself; and otherwise it has two.) Also, there is a new strip $S_{0}=\left(\left\{f_{1}\right\},\left\{f_{2}, \ldots, f_{k-1}\right\},\left\{f_{k}\right\}\right)$. Note that

- for all $j$ with $1 \leq j \leq n, S_{0}$ is parallel or antiparallel with the offspring of $T_{j}$

- for all $i$ with $1 \leq i \leq m$, there exists $j$ with $1 \leq j \leq n$ such that $S_{0}, S_{i}$ disagree on one of the offspring of $T_{j}$, and there exists $j$ such that $S_{0}, S_{i}$ agree on one of the offspring of $T_{j}$. For if the first were false, say, then each of the $T_{j}$ 's has only one offspring, and we could add $f_{1}$ to $A_{i},\left\{f_{2}, \ldots, f_{k-1}\right\}$ to $C_{i}$, and $f_{k}$ to $B_{i}$, contradicting the maximality of the striation; while if the second were false we could do the same with $f_{1}, f_{k}$ exchanged.

- if $T_{1}^{\prime}, T_{2}^{\prime}$ are each offspring of one of $T_{1}, \ldots, T_{n}$, then there exists $i$ with $0 \leq i \leq m$ such that $T_{1}^{\prime}, T_{2}^{\prime}$ agree on $S_{i}$; and there exists $i$ such that they disagree. For this is clear if they are offspring of different parents, since their parents were in a twist together; while if they are both offspring of the same $T_{j}$, then they disagree on $S_{0}$ and agree on all of $S_{1}, \ldots, S_{m}$.

It follows from these observations that the set of strips $S_{0}, \ldots, S_{m}$, together with the set of offspring of $T_{1}, \ldots, T_{n}$, forms a new striation, contrary to the maximality of $L$. This proves (1).

(2) $X$ meets exactly one of $S_{1}, \ldots, S_{m}$.

For by (1) it meets at least one of these sets; suppose it meets two, say $S_{1}$ and $S_{2}$. We may assume that $\left(S_{1}, S_{2}, T_{1}, T_{2}\right)$ is a twist. For $i=1,2$ choose an $S_{i}$-rung $a_{i}$ - $P_{i}$-b $b_{i}$ such that $X$ meets $P_{i}$, and for $j=1,2$ let $x_{j}-Q_{j}-y_{j}$ be a $Q_{j}$-antirung. Then $\left(P_{1}, P_{2}, Q_{1}, Q_{2}\right)$ is a knot $K$ say, and $X \cap V(K)$ is not local with respect to $K$. From the minimality of $F, F$ is minimal such that $X \cap V(K)$ is not local with respect to $K$. It follows from 9.3 that one of 9.3.1, 9.3.4 holds; and in either case there is a vertex $f \in F$ with neighbours in $P_{1}$ and in $P_{2}$. Hence the set of neighbours of $f$ in $V(L)$ is not local with respect to $L$. But this contradicts a hypothesis of the theorem, and hence proves (2).

(3) $V\left(Q_{j}\right) \not X$, for $1 \leq j \leq n$, and for every $T_{j}$-antirung $Q_{j}$.

For suppose that $V\left(Q_{1}\right) \subseteq X$ for some $T_{1}$-antirung $x_{1}-Q_{1}-y_{1}$. By (2) we may assume that $X$ meets $S_{1}$ and none of $S_{2}, \ldots, S_{m}$. Let $2 \leq j \leq n$, and choose $i$ with $2 \leq i \leq m$ such that $\left(S_{1}, S_{i}, T_{1}, T_{j}\right)$ is a twist. Let $Q_{j}$ be an $x_{j}-T_{j}-y_{j}$-antirung, let $a_{1}-P_{1}-b_{1}$ be an $S_{1}$-rung such that $X$ meets $P_{1}$, and let $a_{i}$ - $P_{i}$ - $b_{i}$ be an $S_{i}$-rung. Hence $\left(P_{1}, P_{i}, Q_{1}, Q_{j}\right)$ is a knot. Let us apply 9.3. By (2) and the minimality of $F$ it follows that 9.3 .3 holds. This has several consequences. First, from the minimality of $F, G \mid F$ is an odd path $f_{1} \cdots-f_{k}$ such that $f_{1}, a_{1}$ have the same neighbours in $V\left(Q_{1} \cup Q_{j}\right)$, and so do $f_{k}, b_{1}$, and there are no edges between $F$ and $V\left(P_{1}\right)$ except possibly $f_{1} a_{1}$ and $f_{k} b_{1}$. Since $X$ meets $P_{1}$, it follows that at least one of these two edges is present; and therefore they both are, since $f_{1}-\cdots-f_{k}$ is an odd path and so is $P_{1}$ (for otherwise the union of these two paths, with one of $x_{1}, y_{1}$, would induce an odd hole). So $f_{1}$ is adjacent to $a_{1}$ and to no other vertex of $P_{1}$, and $f_{n}$ to $b_{1}$ and to no other vertex of $P_{1}$. Second, $V\left(Q_{j}\right) \subseteq X$. Since this holds for all $Q_{j}$ it follows that $V\left(T_{j}\right) \subseteq X$; and by exchanging $T_{1}$ and $T_{j}$ we deduce that $V\left(T_{1}\right) \cup \cdots \cup V\left(T_{n}\right) \subseteq X$. Moreover $\left\{f_{2}, \ldots, f_{k-1}\right\}$ is anticomplete to $V\left(T_{1}\right) \cup \cdots \cup V\left(T_{n}\right)$. Third, let $x_{j}^{\prime}-Q_{j}^{\prime}$ - $y_{j}^{\prime}$ be some other $T_{j}$-antirung. By the same argument applied to the knot $\left(P_{1}, P_{i}, Q_{1}, Q_{j}^{\prime}\right)$, we deduce that again 9.3.3 holds, and so one of $f_{1}, f_{k}$ is adjacent to $x_{j}^{\prime}$ and the other to $y_{j}^{\prime}$. Furthermore, the one adjacent to $x_{j}^{\prime}$ is also adjacent to $a_{1}$; 
and so in fact $f_{1}$ is adjacent to $x_{j}^{\prime}$. Since this holds for all choices of $Q_{j}$ and of $j$, it follows that $f_{1}, a_{1}$ have the same neighbours in $V\left(T_{1}\right) \cup \cdots \cup V\left(T_{n}\right)$, and so do $f_{k}, b_{1}$. Hence we can add $f_{1}$ to $A_{1},\left\{f_{2}, \ldots, f_{k-1}\right\}$ to $C_{1}$ and $f_{k}$ to $B_{1}$, contrary to the maximality of the striation. This proves (3).

Since $X$ is not local with respect to $L$, we may assume from (2) and (3) that there exist a vertex of $X \cap V\left(S_{1}\right)$ and a vertex of $X \cap V\left(T_{1}\right)$ that are nonadjacent. By reversing $T_{1}, \ldots, T_{n}$ we may assume that $S_{1}$ is parallel to each $T_{j}$. Since by 9.1 every vertex of $Z_{1}$ is complete to $V\left(S_{1}\right)$, we may assume that there is an $S_{1}$-rung $a_{1}-P_{1}-b_{1}$ and a $T_{1}$-antirung $x_{1}-Q_{1}$ - $y_{1}$ such that $x_{1} \in X$ and $X \cap V\left(P_{1} \backslash a_{1}\right) \neq \emptyset$. Let $2 \leq j \leq n$, and choose $i$ with $2 \leq i \leq m$ such that $\left(S_{1}, S_{i}, T_{1}, T_{j}\right)$ is a twist. Let $P_{i}$ be an $S_{i}$-rung, and let $Q_{j}$ be a $T_{j}$-antirung. So $\left(P_{1}, P_{i}, Q_{1}, Q_{j}\right)$ is a knot $K$ say, and $X \cap V(K)$ is not local with respect to $K$. Let us apply 9.3 ; we deduce that one of the outcomes of 9.3 holds. The first and fourth outcomes contradict (2), and the third contradicts (3), so there is a path with vertex set in $F$ satisfying 9.3.2. From the minimality of $F$, it follows that this path has vertex set $F$, and so $F$ is a path with vertices $f_{1} \cdots-f_{k}$ say. Since $x_{1} \in X$, it follows that one of $f_{1}, f_{k}$ is adjacent to $x_{1}$, and we may assume that $f_{1}$ is adjacent to $x_{1}$. By 9.3.2, $f_{1}$ is also adjacent to $x_{j}$ and to all internal vertices of $Q_{1}, Q_{j}$, and to neither of $y_{1}, y_{j}$, and none of $f_{2}, \ldots, f_{k-1}$ have neighbours in $V\left(Q_{1} \cup Q_{j}\right)$, and $f_{k}$ has a neighbour in $P_{1} \backslash a_{1}$, and $f_{k}$ has no neighbours in $V\left(Q_{1} \cup Q_{j}\right)$. For any other choice of $Q_{j}$ the same happens, and $f_{1}, f_{k}$ cannot become exchanged since $f_{1}$ has neighbours in $Q_{1}$ and $f_{k}$ has none. We deduce that $f_{1}$ is complete to $X_{j} \cup Z_{j}$ and anticomplete to $Y_{j}$; and $\left\{f_{2}, \ldots, f_{k}\right\}$ is anticomplete to $V\left(T_{j}\right)$. In particular there is a vertex of $X \cap V\left(S_{1}\right)$ and a vertex of $X \cap V\left(T_{j}\right)$ that are nonadjacent, and so by exchanging $T_{1}$ and $T_{j}$ in the above argument, we deduce that $f_{1}$ is complete to $X_{1} \cup Z_{1}$ and anticomplete to $Y_{1}$; and $\left\{f_{2}, \ldots, f_{k}\right\}$ is anticomplete to $V\left(T_{1}\right)$. Since this holds for all $j$, it follows that $a_{1}, f_{1}$ have the same neighbours in $V\left(T_{1}\right) \cup \cdots \cup V\left(T_{n}\right)$, and there are no edges between $\left\{f_{2}, \ldots, f_{k}\right\}$ and $V\left(T_{1}\right) \cup \cdots \cup V\left(T_{n}\right)$. But then we can add $f_{1}$ to $A_{1}$ and $\left\{f_{2}, \ldots, f_{k}\right\}$ to $C_{1}$, contrary to the maximality of the striation. This proves 9.5 .

Now we can prove 1.8.3, which we restate.

9.6 Let $G$ be a Berge graph, such that every appearance of $K_{4}$ in $G$ and in $\bar{G}$ is degenerate, and there is no induced subgraph of $G$ isomorphic to $L\left(K_{3,3}\right)$. Then either $G$ is a double split graph, or $G$ admits a balanced skew partition, or one of $G, \bar{G}$ admits a proper 2-join, or there is no appearance of $K_{4}$ in either $G$ or $\bar{G}$.

Proof. If there is an appearance in $G$ of some $K_{4}$-enlargement, say $L\left(H^{\prime}\right)$, then by 5.3 , either $H^{\prime}=K_{3,3}$, which is impossible by hypothesis, or there is a subgraph $H^{\prime \prime}$ of $H^{\prime}$ which is a bipartite subdivision of $K_{4}$, such that $L\left(H^{\prime \prime}\right)$ is nondegenerate, and again this is impossible by hypothesis. So there is no appearance in $G$ of a $K_{4}$-enlargement, and similarly there is none in $\bar{G}$. Moreover, by 7.5 , we may assume that there is no overshadowed appearance of $K_{4}$ in $G$ or in $\bar{G}$. We may assume that there is an appearance of $K_{4}$ in one of $G, \bar{G}$, and consequently $|V(G)| \geq 8$; and by taking complements if necessary we may assume that $L(H)$ is an appearance of $K_{4}$ in $G$. By hypothesis it is degenerate, and hence there is a striation in $G$; choose a maximal striation $L$. Let $L$ have strips $S_{i}=\left(A_{i}, C_{i}, B_{i}\right)(1 \leq i \leq m)$ and antistrips $T_{j}=\left(X_{j}, Z_{j}, Y_{j}\right)(1 \leq j \leq n)$. By 9.4 we can partition $V(G) \backslash V(L)$ into two sets $M, N$, where for every vertex in $M$ its set of neighbours in $V(L)$ is local with respect to $L$, and for every vertex in $N$, its set of neighbours in $V(L)$ resolves $L$.

(1) If there exists $f \in N$ with a nonneighbour in $V\left(S_{1}\right) \cup \cdots \cup V\left(S_{m}\right)$ then the theorem holds. 
For let $f$ have a nonneighbour in $S_{1}$ say. Let $N_{1}$ be the anticomponent of $N$ containing $f$, and let $X$ be the set of all $N_{1}$-complete vertices in $V(G)$. From 9.5 applied in the complement, it follows that $X$ resolves $L$. Since $f$ has a nonneighbour in $V\left(S_{1}\right)$, there is a vertex $u$ of $S_{1}$ not in $X$. Let $U$ be the component of $V(G) \backslash(X \cup N)$ containing $u$. We claim that $U$ is disjoint from $V(L) \backslash V\left(S_{1}\right)$, and no vertex in $V\left(S_{2}\right) \cup \cdots \cup V\left(S_{m}\right)$ has a neighbour in $U$. For suppose not; then there is a path $P$ say in $G$, from $V\left(S_{1}\right)$ to $V(L) \backslash V\left(S_{1}\right)$, with $(X \cup N) \cap V(P) \subseteq V\left(S_{2}\right) \cup \cdots \cup V\left(S_{m}\right)$; choose such a path minimal. It follows that no internal vertex of $P$ is in $V(L)$ or in $X \cup N$; and since $X$ meets every edge between $V\left(S_{1}\right)$ and $V(L) \backslash V\left(S_{1}\right)$, and there are no edges between $V\left(S_{1}\right)$ and $V\left(S_{2}\right) \cup \cdots \cup V\left(S_{m}\right)$, it follows that $P^{*}$ is nonempty. Now no vertex of $P^{*}$ is in $N$, since $N \subseteq N_{1} \cup X$; and so there is a component $M_{1}$ of $M$ including $P^{*}$. From 9.5, the set of attachments of $M_{1}$ in $V(L)$ is local with respect to $L$. Since it has an attachment in $V\left(S_{1}\right)$ it therefore has none in $V\left(S_{2}\right) \cup \cdots \cup V\left(S_{m}\right)$. But the ends of $P$ are attachments of $M_{1}$, they are nonadjacent, and one is in $V\left(S_{1}\right)$ and the other is not, a contradiction. This proves that $U$ is disjoint from $V(L) \backslash V\left(S_{1}\right)$. Let $X^{\prime}$ be the set of vertices in $X$ with neighbours in $U$, and let $V=V(G) \backslash\left(U \cup N_{1} \cup X^{\prime}\right)$. Then $V$ is nonempty because $V\left(S_{2}\right) \subseteq V$; and so $U \cup V, N_{1} \cup X^{\prime}$ is a skew partition of $G$. Since there is a vertex of $S_{2}$ in $X$ (because $X$ resolves $L$ ), and this vertex is in $V$, we deduce that the skew partition is loose, and hence by $4.2 G$ admits a balanced skew partition. This proves (1).

From (1) we may assume that $N$ is complete to $V\left(S_{1}\right) \cup \cdots \cup V\left(S_{m}\right)$, and by taking complements, that $M$ is anticomplete to $V\left(T_{1}\right) \cup \cdots \cup V\left(T_{n}\right)$.

(2) If $M, N$ are both nonempty then the theorem holds.

For let $M_{1}$ be a component of $M$, and $N_{1}$ an anticomponent of $N$. By taking complements we may assume that there is a nonedge between $M_{1}$ and $N_{1}$. Since the set of attachments of $M_{1}$ in $V(L)$ is local by 9.5, and since it has no attachments in $V\left(T_{1}\right) \cup \cdots \cup V\left(T_{n}\right)$, we may assume that all its attachments are in $V\left(S_{1}\right)$. Let $V=V(G) \backslash\left(M_{1} \cup N_{1} \cup V\left(S_{1}\right)\right)$. Since every vertex of $S_{1}$ is $N_{1}$-complete, it follows that $\left(M_{1} \cup V, N_{1} \cup V\left(S_{1}\right)\right)$ is a skew partition of $G$, and since there are $N_{1^{-}}$ complete vertices with no neighbours in $M_{1}$ (for instance, any vertex of $V\left(S_{2}\right)$ ), the skew partition is loose, and by $4.2 G$ admits a balanced skew partition. This proves (2).

\section{(3) If $M, N$ are both empty then the theorem holds.}

For then by 9.1 , we may assume that for $1 \leq j \leq n$ all $Q_{j}$-antirungs have length 1 . If $\left|V\left(S_{1}\right)\right|>2$, then $\left(V\left(S_{1}\right), V(L) \backslash V\left(S_{1}\right)\right)$ is a proper 2-join of $G$; for every vertex in $V\left(T_{1}\right) \cup \cdots \cup V\left(T_{n}\right)$ is either complete to $A_{1}$ and anticomplete to $B_{1} \cup C_{1}$, or complete to $B_{1}$ and anticomplete to $A_{1} \cup C_{1}$ (since all the antirungs have length 1 ). So we may assume that each $S_{i}$ has only two vertices. In particular, every $S_{i}$-rung has length 1 , so by taking complements the same argument shows that we may assume every $V\left(T_{j}\right)$ has only two vertices. But then $G$ is a double split graph and the theorem holds. This proves (3).

From (2) and (3), and taking complements if necessary, we may assume that $N$ is empty and $M$ is nonempty. For $1 \leq i \leq m$ let $M_{i}$ be the union of the components of $M$ that have an attachment in $V\left(S_{i}\right)$, and let $M_{0}$ be the union of the components of $M$ that have no attachments in $V(L)$. 
Then $M_{0}, M_{1}, \ldots, M_{n}$ are pairwise disjoint and have union $M$. If $M_{0}$ is nonempty then $G$ is not connected, and since $|V(G)| \geq 8$ it therefore admits a balanced skew partition, so we may assume that $M_{0}$ is empty. Since $M$ is nonempty we may assume that $M_{1}$ is nonempty. We recall that $T_{1}=\left(X_{1}, Z_{1}, Y_{1}\right)$; suppose that $z \in Z_{1}$. Then $z$ is complete to $V\left(S_{1}\right)$ by 9.1 , and hence if we define $V=V(G) \backslash M_{1} \cup V\left(S_{1}\right) \cup\{z\}$, then $\left(M_{1} \cup V, V\left(S_{1}\right) \cup\{z\}\right)$ is a skew partition of $G$, and by $4.1 G$ admits a balanced skew partition. So we may assume that $Z_{1}$ is empty, and similarly every $Z_{j}$ is empty. Then $\left(M_{1} \cup V\left(S_{1}\right), V(G) \backslash\left(M_{1} \cup V\left(S_{1}\right)\right)\right.$ is a proper 2-join of $G$. This proves 9.6.

It is convenient to combine three earlier results as follows.

9.7 Let $G$ be a Berge graph, such that there is an appearance of $K_{4}$ in $G$. Then either one of $G, \bar{G}$ is a line graph, or $G$ is a double split graph, or one of $G, \bar{G}$ admits a proper 2-join, or $G$ admits a balanced skew partition.

Proof. This is immediate from 9.6, 5.1 and 5.2.

\section{The even prism}

We have completed the first of the main steps of the proof, handling graphs that contain an appearance of $K_{4}$. The next big step is to handle graphs that do not contain an appearance of $K_{4}$, but do contain a long prism. For our purposes, "even" prisms are easier than odd ones, and we treat them in this section. (Odd prisms are treated in sections 11-13.) Incidentally, in this section all we need is that there is no nondegenerate appearance of $K_{4}$ in $G$, and so the results of this section are independent of those in the previous one; these two sections could be in either order. We begin with some results about prisms in general.

For $i=1,2,3$ let $a_{i}-R_{i}-b_{i}$ be a path in $G$, such that these three paths form a prism $K$ with triangles $\left\{a_{1}, a_{2}, a_{3}\right\}$ and $\left\{b_{1}, b_{2}, b_{3}\right\}$. A subset $X \subseteq V(G)$ saturates the prism if at least two vertices of each triangle belong to $X$; and a vertex is major with respect to the prism if its neighbour set saturates it. A subset $X \subseteq V(K)$ is local with respect to the prism if either $X \subseteq V\left(R_{i}\right)$ for some $i$, or $X$ is a subset of one of the triangles. By 7.2, the three paths $R_{1}, R_{2}, R_{3}$ all have lengths of the same parity. A prism is even if the three paths $R_{1}, R_{2}, R_{3}$ have even length, and odd otherwise.

10.1 Let $R_{1}, R_{2}, R_{3}$ form a prism $K$ in a Berge graph $G$, with triangles $\left\{a_{1}, a_{2}, a_{3}\right\}$ and $\left\{b_{1}, b_{2}, b_{3}\right\}$, where each $R_{i}$ has ends $a_{i}$ and $b_{i}$. Let $F \subseteq V(G) \backslash V(K)$ be connected, such that its set of attachments in $K$ is not local. Assume no vertex in $F$ is major with respect to $K$. Then there is a path $f_{1}-\cdots-f_{n}$ in $F$ with $n \geq 1$, such that (up to symmetry) either:

1. $f_{1}$ has two adjacent neighbours in $R_{1}$, and $f_{n}$ has two adjacent neighbours in $R_{2}$, and there are no other edges between $\left\{f_{1}, \ldots, f_{n}\right\}$ and $V(K)$, and (therefore) $G$ has an induced subgraph which is the line graph of a bipartite subdivision of $K_{4}$, or

2. $n \geq 2, f_{1}$ is adjacent to $a_{1}, a_{2}, a_{3}$, and $f_{n}$ is adjacent to $b_{1}, b_{2}, b_{3}$, and there are no other edges between $\left\{f_{1}, \ldots, f_{n}\right\}$ and $V(K)$, or

3. $n \geq 2, f_{1}$ is adjacent to $a_{1}, a_{2}$, and $f_{n}$ is adjacent to $b_{1}, b_{2}$, and there are no other edges between $\left\{f_{1}, \ldots, f_{n}\right\}$ and $V(K)$, or 
4. $f_{1}$ is adjacent to $a_{1}, a_{2}$, and there is at least one edge between $f_{n}$ and $V\left(R_{3}\right) \backslash\left\{a_{3}\right\}$, and there are no other edges between $\left\{f_{1}, \ldots, f_{n}\right\}$ and $V(K) \backslash\left\{a_{3}\right\}$.

Proof. We may assume that $F$ is minimal such that it is connected and its set of attachments in $K$ is not local. Let $X$ be the set of attachments of $F$ in $K$. For $1 \leq i \leq 3$, if $X \cap V\left(R_{i}\right) \neq \emptyset$, let $c_{i}$ and $d_{i}$ be the vertices of $R_{i}$ in $X$ closest (in $R_{i}$ ) to $a_{i}$ and to $b_{i}$ respectively, and let $C_{i}, D_{i}$ be the subpaths of $R_{i}$ between $a_{i}$ and $c_{i}$, and between $d_{i}$ and $b_{i}$ respectively. Let $A=\left\{a_{1}, a_{2}, a_{3}\right\}$ and $B=\left\{b_{1}, b_{2}, b_{3}\right\}$.

We claim that some two-element subset of $X$ is not local. For since $X \nsubseteq B$ we may assume that $c_{1}$ exists and $c_{1} \neq b_{1}$. Since $X \nsubseteq V\left(R_{1}\right)$, we may assume $d_{2}$ exists. If $d_{2} \neq a_{2}$ then $\left\{c_{1}, d_{2}\right\}$ is the desired subset; so we may assume $d_{2}=a_{2}$, and similarly $d_{3}=a_{3}$ if $d_{3}$ exists. Since $X \nsubseteq A$, it follws that $d_{1} \neq a_{1}$, and then $\left\{a_{2}, d_{1}\right\}$ is the desired subset. So some two-element subset $\left\{x_{1}, x_{2}\right\}$ of $X$ is not local. Consequently $x_{1}, x_{2}$ are not adjacent. From the minimality of $F$, there is a path with vertices $x_{1}, f_{1}, \ldots, f_{n}, x_{2}$ such that $F=\left\{f_{1}, \ldots, f_{n}\right\}$.

(1) If $n=1$ then the theorem holds.

For assume $n=1$; then $F=\left\{f_{1}\right\}$. Since $X$ is not local it meets at least two of the paths; suppose it only meets $R_{1}$ and $R_{2}$. Suppose that $c_{1}=d_{1}$. Then we may assume that $c_{1} \notin A$ and $c_{2} \neq b_{2}$, by exchanging $A$ and $B$ if necessary; but then $c_{1}$ can be linked onto the triangle $A$, via the paths $c_{1}-C_{1}-a_{1}$, $c_{1}-f_{1}-c_{2}-C_{2}-a_{2}$, and $c_{1}-D_{1}-b_{1}-b_{3}-R_{3}-a_{3}$, contrary to 2.4 , since $f$ has at most one neighbour in $A$. So $c_{1}$ is different from $d_{1}$, and similarly $c_{2}$ is different from $d_{2}$ (and in particular, $c_{2} \neq b_{2}$ ). Suppose that $c_{1}$ is nonadjacent to $d_{1}$. Then since $f_{1}$ is not major, we may assume it has at most one neighbour in $A$, by exchanging $A$ and $B$ if necessary; but it can be linked onto $A$, via $f_{1}-c_{1}-C_{1}-a_{1}, f_{1}-c_{2}-C_{2}-a_{2}$ and $f_{1}-d_{1}-D_{1}-b_{1}-b_{3}-R_{3}-a_{3}$, contrary to 2.4 . So $c_{1}, d_{1}$ are adjacent, and similarly so are $c_{2}, d_{2}$, but then statement 1 of the theorem holds. So we may assume that $X$ meets all three of $R_{1}, R_{2}, R_{3}$. Since $f_{1}$ is not major, we may assume that it has at most one neighbour in $A$, by exchanging $A$ and $B$ if necessary, and therefore cannot be linked onto $A$. Since it has neighbours in all three of $R_{1}, R_{2}, R_{3}$, it follows that for at least two of these paths, the only neighbour of $f_{1}$ in this path is in $B$. We may assume therefore that $c_{1}=b_{1}$ and $c_{2}=b_{2}$. Since $X$ is not local, $c_{3} \neq b_{3}$; but then statement 4 of the theorem holds. This proves (1).

We may therefore assume that $n \geq 2$. Let $X_{1}$ be the set of attachments of $F \backslash\left\{f_{1}\right\}$, and $X_{2}$ the set of attachments of $F \backslash\left\{f_{n}\right\}$. From the minimality of $F$, both $X_{1}$ and $X_{2}$ are local. Moreover, $X=X_{1} \cup X_{2}$, and for $2 \leq i \leq n-1$, every neighbour of $f_{i}$ in $K$ belongs to $X_{1} \cap X_{2}$.

(2) If $X_{1} \subseteq A$ and $X_{2} \subseteq V\left(R_{1}\right)$ then the theorem holds.

For then $f_{1}$ has at least one neighbour in $R_{1} \backslash a_{1}$, and $f_{n}$ is adjacent to at least one of $a_{2}, a_{3}$, and there are no other edges between $F$ and $V(K) \backslash\left\{a_{1}\right\}$. If $f_{n}$ is adjacent to both $a_{2}, a_{3}$ then statement 4 of the theorem holds, so we assume it is not adjacent to $a_{3}$. But then $a_{2}$ can be linked onto the triangle $B$, via $a_{2^{-}} f_{n^{-}} f_{n-1^{-}} \cdots-f_{1}-d_{1}-D_{1}-b_{1}, a_{2}-R_{2}-b_{2}, a_{2}-a_{3}-R_{3}-b_{3}$, contrary to 2.4 . This proves (2).

From (2), since both $X_{1}$ and $X_{2}$ are local, we may assume that either $X_{1} \subseteq A$ and $X_{2} \subseteq B$, or $X_{1} \subseteq V\left(R_{2}\right)$ and $X_{2} \subseteq V\left(R_{1}\right)$. In either case $X_{1} \cap X_{2}=\emptyset$, so none of $f_{2}, \ldots, f_{n-1}$ has any neighbours 
in $V(K)$. Therefore $X_{1}$ is the set of neighbours of $f_{n}$ in $V(K)$, and $X_{2}$ is the set of neighbours of $f_{1}$ in $V(K)$.

(3) If $X_{1} \subseteq A$ and $X_{2} \subseteq B$ then the theorem holds.

For then we may assume that $f_{n}$ is adjacent to $a_{1}$ and $f_{1}$ to $b_{2}$. Suppose first that $n$ has the same parity as the length of $R_{1}$. Since $a_{2}-R_{2}-b_{2^{-}} f_{1^{-}} \cdots-f_{n^{-}} a_{2}$ is not an odd hole, it follows that $f_{n}$ is not adjacent to $a_{2}$, and similarly $f_{1}$ is not adjacent to $b_{1}$. Since $a_{3}-R_{3}-b_{3}-b_{2}-f_{1}-\cdots-f_{n}-a_{1}-a_{3}$ is not an odd hole, either $f_{n}$ is adjacent to $a_{3}$ or $f_{1}$ to $b_{3}$, and not both, as we saw before. But then statement 4 of the theorem holds. Now suppose that $n$ has different parity from the length of $R_{1}$. Since $a_{1}-a_{2}-R_{2}-b_{2}-f_{1}-\cdots-f_{n}-a_{1}$ is not an odd hole, $f_{n}$ is adjacent to $a_{2}$, and similarly $f_{1}$ to $b_{1}$. If there are no more edges between $F$ and $V(K)$ then statement 3 of the theorem holds, so we may assume that $f_{n}$ is adjacent to $a_{3}$. By the same argument as before it follows that $f_{1}$ is adjacent to $b_{3}$, and then statement 2 of the theorem holds. This proves (3).

From (2) and (3) we may assume that $X_{1} \subseteq V\left(R_{2}\right)$ and $X_{2} \subseteq V\left(R_{1}\right)$. So $f_{1}$ is adjacent to the vertices of $R_{1}$ that are in $X$, and $f_{n}$ to those of $R_{2}$ in $X$. If $c_{1}=d_{1}$, then from the symmetry we may assume that $c_{1} \neq a_{1}$, and $c_{2} \neq b_{2}$; but then $c_{1}$ can be linked onto $A$, via $c_{1}-C_{1}-a_{1}$, $c_{1}-f_{1^{-}} \cdots-f_{n^{-}}-c_{2}-C_{2}-a_{2}, c_{1}-D_{1}-b_{1}-b_{3}-R_{3}-a_{3}$, contrary to 2.4 . So $c_{1} \neq d_{1}$ and similarly $c_{2} \neq d_{2}$; and in particular $c_{2} \neq b_{2}$. If $c_{1}, d_{1}$ are nonadjacent, then $f_{1}$ can be linked onto $A$ via $f_{1}-c_{1}-C_{1}-a_{1}$, $f_{1}-\cdots-f_{n^{-}} c_{2}-C_{2}-a_{2}, f_{1}-d_{1}-D_{1}-b_{1}-b_{3}-R_{3}-a_{3}$; but $f_{1}$ has at most one neighbour in $A$ (because $n \geq 2$ ), contrary to 2.4. So $c_{1}, d_{1}$ are adjacent, and similarly so are $c_{2}, d_{2}$; but then statement 1 of the theorem holds. This proves 10.1 .

10.2 Let $R_{1}, R_{2}, R_{3}, K, F$ be as in 10.1, and suppose that 10.1.1 holds. Then either $R_{1}$ and $R_{2}$ both have length 1 , or there is a nondegenerate appearance of $K_{4}$ in $G$.

Proof. For let $f_{1^{-}} \cdots-f_{n}$ be a path in $F$ such that $f_{1}$ has two adjacent neighbours in $R_{1}$, and $f_{n}$ has two adjacent neighbours in $P_{2}$, and there are no other edges between $\left\{f_{1}, \ldots, f_{n}\right\}$ and $V(K)$. Then $G \mid\left(V(K) \cup\left\{f_{1}, \ldots, f_{n}\right\}\right.$ is a line graph of a bipartite subdivision of $K_{4}$. We may assume it is degenerate. Hence the prism is odd, for all prisms contained in a degenerate appearance of $K_{4}$ are odd. So $R_{3}$ is odd, and therefore so is the path $f_{1^{-}} \cdots-f_{n}$, and the other four "rungs" of this line graph have length 0 . In particular, $R_{1}$ and $R_{2}$ both have length 1 . This proves 10.2 .

There is also a tighter version of 10.1, the following.

10.3 Let $G$ be a Berge graph, such that there is no nondegenerate appearance of $K_{4}$ in $G$. Let $R_{1}, R_{2}, R_{3}$ form a prism $K$ in $G$, with triangles $\left\{a_{1}, a_{2}, a_{3}\right\}$ and $\left\{b_{1}, b_{2}, b_{3}\right\}$, where each $R_{i}$ has ends $a_{i}$ and $b_{i}$. Let $F \subseteq V(G) \backslash V(K)$ be connected, such that no vertex in $F$ is major with respect to $K$. Let $x_{1}$ be an attachment of $F$ in the interior of $R_{1}$, and assume that there is another attachment $x_{2}$ of $F$ not in $R_{1}$. Then there is a path $f_{1}-\cdots-f_{n}$ in $F$ such that (up to the symmetry between $A$ and B) $f_{1}$ is adjacent to $a_{2}, a_{3}$, and $f_{n}$ has at least one neighbour in $R_{1} \backslash a_{1}$, and there are no other edges between $\left\{f_{1}, \ldots, f_{n}\right\}$ and $V(K) \backslash\left\{a_{1}\right\}$.

Proof. We may assume $F$ is minimal such that it is connected, $x_{1}$ is one of its attachments, and it has some attachment $x_{2}$ in $R_{2} \cup R_{3}$. Hence there is a path $x_{2}-v_{1^{-}} \cdots-v_{m}-x_{1}$ where $F=\left\{v_{1}, \ldots, v_{m}\right\}$. By 
10.1, there is a subpath $f_{1^{-}} \cdots-f_{n}$ of $v_{1}-\cdots-v_{m}$ such that one of 10.1.1-4 holds. From the minimality of $F, v_{1}$ is the only vertex of $F$ with a neighbour in $V\left(R_{2}\right) \cup V\left(R_{3}\right)$, and in particular, at most one vertex of $f_{1} \cdots-f_{n}$ has a neighbour in $V\left(R_{2}\right) \cup V\left(R_{3}\right)$. We deduce that $f_{1^{-}} \cdots-f_{n}$ does not satisfy 10.1.2 or 10.1.3. Suppose it satisfies 10.1.1. By 10.2 the path $f_{1^{-}} \cdots-f_{n}$ joins two of $R_{1}, R_{2}, R_{3}$ that are both of length 1 , and therefore $n$ is even. Since $R_{1}$ has length $\geq 2$ (because $x_{1}$ is in its interior) it follows that $f_{1}, f_{n}$ are distinct vertices of $F$ both with neighbours in $V\left(R_{2}\right) \cup V\left(R_{3}\right)$, a contradiction. So $f_{1}-\cdots-f_{n}$ satisfies 10.1.4, and therefore we may assume that for some $i$ with $1 \leq i \leq 3, f_{1}$ is adjacent to the two vertices in $A \backslash\left\{a_{i}\right\}$, and $f_{n}$ has at least one neighbour in $R_{i} \backslash a_{i}$, and there are no other edges between $\left\{f_{1}, \ldots, f_{n}\right\}$ and $V(K) \backslash\left\{a_{i}\right\}$. Suppose first that $i>1, i=2$ say. Then both $f_{1}, f_{n}$ have neighbours in $V\left(R_{2}\right) \cup V\left(R_{3}\right)$, and so from the minimality of $F$ it follows that $n=1$ and $f_{1}=v_{1}$. But then $f_{1}$ can be linked onto the triangle $B$, via the path between $f_{1}$ and $b_{1}$ with interior in $\left\{v_{2}, \ldots, v_{m}\right\} \cup V\left(R_{1} \backslash a_{1}\right)$, the path between $f_{1}$ and $b_{2}$ with interior in $V\left(R_{2} \backslash a_{2}\right)$, and the path $f_{1}-a_{3}-R_{3}-b_{3}$, contrary to 2.4 . Hence $i=1$, and the theorem is satisfied. This proves 10.3 .

Another useful corollary of 10.1 is the following.

10.4 Let $G$ be Berge, such that there is no nondegenerate appearance of $K_{4}$ in $G$. Let $R_{1}, R_{2}, R_{3}$ form a prism $K$ in a Berge graph $G$, with triangles $\left\{a_{1}, a_{2}, a_{3}\right\}$ and $\left\{b_{1}, b_{2}, b_{3}\right\}$, where each $R_{i}$ has ends $a_{i}$ and $b_{i}$. Let $F \subseteq V(G) \backslash V(K)$ be connected, such that if the prism is even then no vertex in $F$ is major with respect to $K$. Assume that the set of attachments of $F$ in $K$ is not local, but none are in $V\left(R_{3}\right)$. Then $|F| \geq 2$, and the set of attachments of $F$ in $K$ is precisely $\left\{a_{1}, b_{1}, a_{2}, b_{2}\right\}$.

Proof. If there is a major vertex $v \in F$, then since it has no neighbours in $R_{3}$, it is adjacent to $a_{1}$ and $b_{2}$, and since $v-a_{1}-a_{3}-R_{3}-b_{3}-b_{2}-v$ is a hole, it follows that the prism is even, contrary to the hypothesis. So there is no major vertex in $F$. By 10.3 no internal vertex of $R_{1}$ or $R_{2}$ is an attachment of $F$. By 10.1, there is a path in $F$ satisfying one of 10.1.1-4; and since it has no attachments in $R_{3}$, it must satisfy 10.1 .1 or 10.1 .3 , and in either case $a_{1}, b_{1}, a_{2}, b_{2}$ are all attachments of $F$. Since no vertex in $F$ is major it follows that $|F| \geq 2$. This proves 10.4 .

The next result is a close relative of 7.5 .

10.5 Let $G$ be Berge, such that there is no nondegenerate appearance of $K_{4}$ in $G$. If there is an even prism $K$ in $G$, such that some vertex of $G$ is major with respect to $K$, then $G$ admits a balanced skew partition.

Proof. Any prism has six vertices of degree 3, called triangle-vertices; choose a prism $K$ and a nonempty anticonnected set $Y \subseteq V(G) \backslash V(K)$, such that every vertex in $Y$ is major with respect to the prism, and as few triangle-vertices of $K$ are $Y$-complete as possible. Let the paths $a_{i}-R_{i}-b_{i}(i=1,2,3)$ form $K$, where $\left\{a_{1}, a_{2}, a_{3}\right\},\left\{b_{1}, b_{2}, b_{3}\right\}$ are its triangles. We may assume that $Y$ is maximal with the given property. Let $X$ be the set of all $Y$-complete vertices in $G$. By $7.3, X$ saturates $K$. Consequently there is one of $R_{1}, R_{2}, R_{3}$ with both ends in $X$, say $R_{1}$. Let $X_{0}=X \backslash V(K)$ and $X_{1}=\left\{a_{1}, b_{1}\right\}$.

(1) If $F \subseteq V(G)$ is connected and some vertex of $V\left(R_{1}^{*}\right)$ has a neighbour in $F$, and so does some vertex of $V\left(R_{2}\right) \cup V\left(R_{3}\right)$, then $F \cap\left(X_{0} \cup X_{1} \cup Y\right)$ is nonempty. 
Suppose for a contradiction that some $F$ exists not satisfying (1), and choose it minimal. Hence $G \mid F$ is a path, disjoint from $K$. Consequently $F \cap X=\emptyset$. Suppose some vertex in $v \in F$ is major with respect to $K$. Then since $v \notin X$ it follows that $v$ has a nonneighbour in $Y$, and so $Y \cup\{v\}$ is anticonnected; the maximality of $Y$ therefore implies that $v \in Y$, and hence $F \cap Y \neq \emptyset$ and the claim holds. So we may assume that no vertex in $F$ is major. Let $x_{1}$ be an attachment of $F$ in $R_{1}^{*}$. By 10.3 we may assume that there is a path $f_{1^{-}} \cdots-f_{n}$ in $F$ such that $f_{1}$ is adjacent to $a_{2}, a_{3}$, and $f_{n}$ has neighbours in $R_{1} \backslash a_{1}$, and $f_{1} a_{2}, f_{1} a_{3}$ are the only edges between $\left\{f_{1}, \ldots, f_{n}\right\}$ and $V\left(R_{2}\right) \cup V\left(R_{3}\right)$ Now there is a path $R$ from $f_{1}$ to $b_{1}$ with interior in $\left\{f_{2}, \ldots, f_{n}\right\} \cup V\left(R_{1} \backslash a_{1}\right)$, and hence $R, R_{2}, R_{3}$ form a prism $K^{\prime}$ say. By 7.4, every vertex in $Y$ is major with respect to $K^{\prime}$, and since $a_{1}$ is $Y$-complete and $f_{1}$ is not, it follows that the number of $Y$-complete triangle-vertices in $K^{\prime}$ is smaller than the number in $K$, a contradiction. This proves (1).

It follows from (1) that there is a partition of $V(G) \backslash\left(X_{0} \cup X_{1} \cup Y\right)$ into two sets $L$ and $M$ say, where there is no edge between $L$ and $M$, and $V\left(R_{1}^{*}\right) \subseteq L$ and $V\left(R_{2}\right) \cup V\left(R_{3}\right) \subseteq M$. So $\left(L \cup M, X_{0} \cup X_{1} \cup Y\right)$ is a skew partition of $G$. Since at least two vertices of $A$ are in $X$ and only one is in $X_{1}$, there is a vertex of $X$ in $M$, and so the skew partition is loose. By 4.2 the result follows. This proves 10.5 .

The main result of this section is 1.8 .4 , which we restate.

10.6 Let $G$ be a Berge graph, such that there is no nondegenerate appearance of $K_{4}$ in $G$. If $G$ contains an even prism, then either $G$ is an even prism with $|V(G)|=9$, or $G$ admits a proper 2-join or a balanced skew partition.

Proof. Since $G$ contains an even prism, we can choose in $G$ a collection of nine sets

$$
\begin{array}{lll}
A_{1} & C_{1} & B_{1} \\
A_{2} & C_{2} & B_{2} \\
A_{3} & C_{3} & B_{3}
\end{array}
$$

with the following properties:

- all these sets are nonempty and pairwise disjoint

- for $1 \leq i<j \leq 3, A_{i}$ is complete to $A_{j}$ and $B_{i}$ is complete to $B_{j}$, and there are no other edges between $A_{i} \cup B_{i} \cup C_{i}$ and $A_{j} \cup B_{j} \cup C_{j}$

- for $1 \leq i \leq 3$, every vertex of $A_{i} \cup B_{i} \cup C_{i}$ belongs to a path between $A_{i}$ and $B_{i}$ with interior in $C_{i}$

- some path between $A_{1}$ and $B_{1}$ with interior in $C_{1}$ is even.

We call this collection of nine sets a hyperprism. Let $H$ be the subgraph of $G$ induced on the union of the nine sets. Choose the hyperprism with $V(H)$ maximal. For $1 \leq i \leq 3$, a path from $A_{i}$ to $B_{i}$ with interior in $C_{i}$ is called an $i$-rung. Let us write $S_{i}=A_{i} \cup B_{i} \cup C_{i}$ for $1 \leq i \leq 3$, and $A=A_{1} \cup A_{2} \cup A_{3}$, and $B=B_{1} \cup B_{2} \cup B_{3}$.

(1) For $1 \leq i \leq 3$, all $i$-rungs have even length. 
For we are given that some 1-rung $R_{1}$ say has even length. Let $R_{2}$ be an 2-rung; then the union of $R_{1}$ and $R_{2}$ induces a hole, and so $R_{2}$ is even. Hence every 2- or 3-rung is even, and hence so is every 1-rung. This proves (1).

A subset $X \subseteq V(H)$ is local (with respect to the hyperprism) if $X$ is a subset of one of $S_{1}, S_{2}, S_{3}, A$ or $B$.

(2) We may assume that for every connected subset $F$ of $V(G) \backslash V(H)$, its set of attachments in $H$ is local.

For suppose not. Choose $F$ minimal, and let $X$ be the set of attachments of $F$ in $H$. Suppose first that there exists $x_{1} \in X \cap C_{1}$. Since $X$ is not local, we may assume that there exists $x_{2} \in X \cap S_{2}$. For $i=1,2,3$ choose an $i$-rung $R_{i}$ with ends $a_{i} \in A_{i}$ and $b_{i} \in B_{i}$, such that $x_{i} \in V\left(R_{i}\right)$ for $i=1,2$. Then $R_{1}, R_{2}, R_{3}$ form an even prism $K$ say. By 10.5 we may assume no vertex in $F$ is major with respect to $K$; so by 10.3 , we may assume that there is a path $f_{1}-\cdots-f_{n}$ in $F$ such that $f_{1}$ is adjacent to $a_{2}, a_{3}$, and $f_{n}$ has at least one neighbour in $R_{1} \backslash a_{1}$, and there are no other edges between $\left\{f_{1}, \ldots, f_{n}\right\}$ and $V(K) \backslash\left\{a_{1}\right\}$. From the minimality of $F$ it follows that $F=\left\{f_{1}, \ldots, f_{n}\right\}$. Since this holds for all choices of $R_{3}$ it follows that $f_{1}$ is complete to $A_{3}$ and there are no edges between $\left\{f_{1}, \ldots, f_{n}\right\}$ and $B_{3} \cup C_{3}$. Since $a_{3} \in X$ the same conclusion follows for all choices of $R_{2}$, and so $f_{1}$ is complete to $A_{2}$ and there are no edges between $\left\{f_{1}, \ldots, f_{n}\right\}$ and $B_{2} \cup C_{2}$. But then we can add $f_{1}$ to $A_{1}$ and $\left\{f_{2}, \ldots, f_{n}\right\}$ to $C_{1}$, contradicting the maximality of the hyperprism.

It follows that $X \cap C_{1}=\emptyset$, and similarly $X \cap C_{2}, X \cap C_{3}=\emptyset$. We claim there is a 2-element subset of $X$ which is also not local. For we may assume $X \cap A_{1} \neq \emptyset$; and hence if $X$ meets $B_{2}$ or $B_{3}$ our claim holds. If not, then it meets $B_{1}$ (since it is not a subset of $A$ ) and meets $A_{2} \cup A_{3}$ (since it is not a subset of $S_{1}$ ), and again the claim holds. So there is a subset $\left\{x_{1}, x_{2}\right\}$ of $X$ which is not local. We may assume that $x_{1} \in A_{1}$ and $x_{2} \in B_{2}$. From the minimality of $F$, there is a path $x_{1}-f_{1^{-}} \cdots-f_{n}-x_{2}$ with $F=\left\{f_{1}, \ldots, f_{n}\right\}$.

Suppose first that $n$ is even. For any 3 -rung $R_{3}$ with ends $a_{3} \in A_{3}$ and $b_{3} \in B_{3}$,

$$
x_{1}-f_{1}-\cdots-f_{n}-x_{2}-b_{3}-R_{3}-a_{3}-x_{1}
$$

is not an odd hole, and so some vertex of $R_{3}$ is in $X$. Since $X \cap C_{3}=\emptyset$, and $a_{3}$ has no neighbour in $\left\{f_{2}, \ldots, f_{n}\right\}$ from the minimality of $F$, and similarly $b_{3}$ has no neighbour in $\left\{f_{1}, \ldots, f_{n-1}\right\}$, it follows that either $f_{1}$ is adjacent to $a_{3}$, or $f_{n}$ to $b_{3}$ (and not both, since otherwise $f_{1}-\cdots-f_{n}-b_{3}-R_{3}-a_{3}$ is an odd hole). From the symmetry we may assume that $f_{n}$ is adjacent to $b_{3}$. By exchanging $S_{2}$ and $S_{3}$ it follows that for every 2-rung with ends $a_{2} \in A_{2}$ and $b_{2} \in B_{2}$, either $f_{1}$ is adjacent to $a_{2}$ or $f_{n}$ to $b_{2}$, and not both. Suppose that $f_{n}$ is complete to $B_{2} \cup B_{3}$; then $f_{1}$ has no neighbours in $S_{2} \cup S_{3}$, and we can add $f_{n}$ to $B_{1}$ and $f_{1}, \ldots, f_{n-1}$ to $C_{1}$, contrary to the maximality of the hyperprism. So $f_{n}$ is not complete to $B_{2} \cup B_{3}$, and hence $f_{1}$ has a neighbour in one of $A_{2}, A_{3}$, say $A_{3}$; and by exchanging $S_{1}$ and $S_{2}$ it follows that for every 1-rung with ends $a_{1} \in A_{1}$ and $b_{1} \in B_{1}$, either $f_{1}$ is adjacent to $a_{1}$ or $f_{n}$ to $b_{1}$ and not both. In particular, $f_{1}$ has no neighbours in $B$ and $f_{n}$ has none in $A$. For $i=1,2,3$ let $A_{i}^{\prime}$ be the set of neighbours of $f_{1}$ in $A_{i}$, and let $A_{i}^{\prime \prime}=A_{i} \backslash A_{i}^{\prime}$; let $B_{i}^{\prime \prime}$ be the set of neighbours of $f_{n}$ in $B_{i}$, and let $B_{i}^{\prime}=B_{i} \backslash B_{i}^{\prime \prime}$. We have shown so far that every $i$-rung is either between $A_{i}^{\prime}$ and $B_{i}^{\prime}$ or between $A_{i}^{\prime \prime}$ and $B_{i}^{\prime \prime}$. Let $C_{i}^{\prime}$ be the union of the interiors of the $i$-rungs between $A_{i}^{\prime}$ and $B_{i}^{\prime}$, 
and $C_{i}^{\prime \prime}$ the union of the interiors of the $i$-rungs between $A_{i}^{\prime \prime}$ and $B_{i}^{\prime \prime}$. We observe that $C_{i}=C_{i}^{\prime} \cup C_{i}^{\prime \prime}$. Moreover, $C_{i}^{\prime} \cap C_{i}^{\prime \prime}=\emptyset$, for otherwise there would be an $i$-rung between $A_{i}^{\prime}$ and $B_{i}^{\prime \prime}$. For the same reason there are no edges between $A_{i}^{\prime} \cup C_{i}^{\prime}$ and $C_{i}^{\prime \prime} \cup B_{i}^{\prime \prime}$, and no edges between $A_{i}^{\prime \prime} \cup C_{i}^{\prime \prime}$ and $C_{i}^{\prime} \cup B_{i}^{\prime}$. We claim that $A_{i}^{\prime}$ is complete to $A_{i}^{\prime \prime}$. For if not, let $R^{\prime \prime}$ be an $i$-rung with ends $a^{\prime \prime} \in A_{i}^{\prime \prime}$ and $b^{\prime \prime} \in B_{i}^{\prime \prime}$, and let $a^{\prime} \in A_{i}^{\prime}$ be nonadjacent to $a^{\prime \prime}$. Since we have seen that $f_{n}$ has neighbours in at least two of $B_{1}, B_{2}, B_{3}$, it follows that at least two of $A_{1}^{\prime \prime}, A_{2}^{\prime \prime}, A_{3}^{\prime \prime}$ are nonempty, and therefore we may choose $a \in A_{j}^{\prime \prime}$ for some $j \neq i$. Then

$$
a-a^{\prime}-f_{1^{-}} \cdots-f_{n^{-}}-b^{\prime \prime}-R^{\prime \prime}-a^{\prime \prime}-a
$$

is an odd hole, a contradiction. So $A_{i}^{\prime}$ is complete to $A_{i}^{\prime \prime}$ for each $i$, and similarly $B_{i}^{\prime}$ is complete to $B_{i}^{\prime \prime}$ for each $i$. We showed already that we may assume that $A_{1}^{\prime}, A_{2}^{\prime \prime}, A_{3}^{\prime}, A_{3}^{\prime \prime}$ are all nonempty. But then the nine sets

$$
\begin{array}{ccc}
A_{1}^{\prime} & C_{1}^{\prime} & B_{1}^{\prime} \\
A_{2}^{\prime} \cup A_{3}^{\prime} & C_{2}^{\prime} \cup C_{3}^{\prime} & B_{2}^{\prime} \cup B_{3}^{\prime} \\
A_{1}^{\prime \prime} \cup A_{2}^{\prime \prime} \cup A_{3}^{\prime \prime} \cup\left\{f_{1}\right\} & C_{1}^{\prime \prime} \cup C_{2}^{\prime \prime} \cup C_{3}^{\prime \prime} \cup\left\{f_{2}, \ldots, f_{n}\right\} & B_{1}^{\prime \prime} \cup B_{2}^{\prime \prime} \cup B_{3}^{\prime \prime}
\end{array}
$$

form a hyperprism, contrary to the maximality of $V(H)$. This completes the argument when $n$ is even.

Now assume $n$ is odd. $f_{1}$ has a neighbour $a_{1}$ say in $A_{1}$; let $R_{1}$ be a 1-rung with ends $a_{1}$ and $b_{1}$ say. Similarly let $R_{2}$ be a 2-rung with ends $a_{2}$ and $b_{2}$, where $b_{2} \in B_{2}$ is adjacent to $f_{n}$. Since $a_{1}-f_{1}-\cdots-f_{n}-b_{2}-b_{1}-R_{1}-a_{1}$ is not an odd hole, it follows that $b_{1} \in X$, and similarly $a_{2} \in X$. From the minimality of $F$, one of $b_{1}, a_{2}$ is adjacent to $f_{1}$ and the other to $f_{n}$, and neither has any more neighbours in $F$. Suppose that $f_{n}$ is not adjacent to $b_{1}$; so $f_{1}$ is adjacent to $b_{1}$, and $n \geq 2$, and $f_{n}$ is adjacent to $a_{2}$. But then $b_{1}-f_{1}-\cdots-f_{n}-b_{2}-b_{1}$ is an odd hole, a contradiction. This proves that $f_{n}$ is adjacent to $b_{1}$ and $f_{1}$ to $a_{2}$. Hence for all $1 \leq i \leq 3$, and for every $i$-rung with ends $a \in A$ and $b \in B, a \in X$ if and only if $b \in X$, and if so then $f_{1}$ is adjacent to $a$ and $f_{n}$ to $b$. Consequently, for every vertex in $X \cap A, f_{1}$ is its unique neighbour in $F$, and for every vertex in $X \cap B, f_{n}$ is its unique neighbour in $F$. For $1 \leq i \leq 3$, let

$$
\begin{aligned}
A_{i}^{\prime} & =A_{i} \cap X \\
B_{i}^{\prime} & =B_{i} \cap X \\
A_{i}^{\prime \prime} & =A_{i} \backslash X \\
B_{i}^{\prime \prime} & =B_{i} \backslash X .
\end{aligned}
$$

Let $C_{i}^{\prime}$ be the union of the interior of the $i$-rungs between $A_{i}^{\prime}$ and $B_{i}^{\prime}$, and $C_{i}^{\prime \prime}$ the union of the interior of the $i$-rungs between $A_{i}^{\prime \prime}$ and $B_{i}^{\prime \prime}$. We have seen that every $i$-rung is of one of these two types, and so $C_{i}=C_{i}^{\prime} \cup C_{i}^{\prime \prime}$. Moreover, since there is no rung between $A_{i}^{\prime}$ and $B_{i}^{\prime \prime}$, it follows that $C_{i}^{\prime} \cap C_{i}^{\prime \prime}=\emptyset$, and there are no edges between $A_{i}^{\prime} \cup C_{i}^{\prime}$ and $C_{i}^{\prime \prime} \cup B_{i}^{\prime \prime}$, and similarly no edges between $A_{i}^{\prime \prime} \cup C_{i}^{\prime \prime}$ and $C_{i}^{\prime} \cup B_{i}^{\prime}$. We have seen that $f_{1}$ has neighbours in at least two of $A_{1}, A_{2}, A_{3}$, and $f_{n}$ has neighbours in at least two of $B_{1}, B_{2}, B_{3}$. We claim that also $f_{1}$ has nonneighbours in at least two of $A_{1}, A_{2}, A_{3}$, and the same for $f_{n}$. For suppose not, and $f_{1}$ is complete to $A_{1} \cup A_{2}$ say. Then $f_{n}$ is complete to $B_{1} \cup B_{2}$; by 10.5 we may assume that $n>1$, and so we can add $f_{1}$ to $A_{3}, f_{n}$ to $B_{3}$ and $f_{2}, \ldots, f_{n-1}$ to $C_{3}$, contrary to the maximality of $V(H)$. This proves that $f_{1}$ has nonneighbours in at least two of $A_{1}, A_{2}, A_{3}$, and similarly $f_{n}$ has nonneighbours in at least two of $B_{1}, B_{2}, B_{3}$. Let $1 \leq i \leq 3$; we claim that $A_{i}^{\prime}$ is complete to $A_{i}^{\prime \prime}$. For we may assume that $i=1$; suppose that $a^{\prime} \in A_{1}^{\prime}$ and $a^{\prime \prime} \in A_{1}^{\prime \prime}$ 
are nonadjacent, and let $R^{\prime \prime}$ be a 1-rung with ends $a^{\prime \prime}, b^{\prime \prime}$. Choose $a \in A_{2}^{\prime \prime} \cup A_{3}^{\prime \prime}$ and $b \in B_{2}^{\prime} \cup B_{3}^{\prime}$; then $a, b$ are not adjacent since all rungs have even length, and so $a-a^{\prime}-f_{1^{-}} \cdots-f_{n^{-}}-b-b^{\prime \prime}-R^{\prime \prime}-a^{\prime \prime}-a$ is an odd hole, a contradiction. This proves that $A_{i}^{\prime}$ is complete to $A_{i}^{\prime \prime}$ for $i=1,2,3$, and similarly $B_{i}^{\prime}$ is complete to $B_{i}^{\prime \prime}$. We have seen that we may assume that $A_{1}^{\prime}, A_{2}^{\prime}$ are nonempty. But then

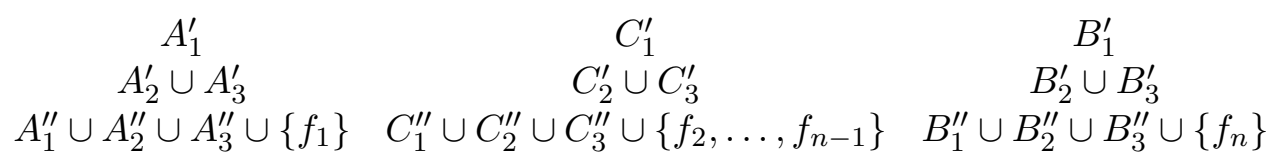

is a hyperprism, contrary to the maximality of $V(H)$. This proves (2).

Suppose $F$ is a component of $V(G) \backslash V(H)$, and all its attachments are in $A$. Then $(V(G) \backslash A, A)$ is a skew partition of $G$. We must show that $G$ admits a balanced skew partition. Choose $b_{2} \in B_{2}$ and $a_{3} \in A_{3}$. Then $B_{1} \cup C_{1} \cup\left\{b_{2}\right\}$ is connected, and all vertices in $A_{1}$ have neighbours in it. By 2.6, $\left(B_{1} \cup C_{1} \cup\left\{b_{2}\right\}, A_{1}\right)$ is balanced, and so by 2.7 .1 , so is $\left(A_{1}, F\right)$. By $4.5, G$ admits a balanced skew partition. So we may assume there is no such $F$, and the same for $B$.

From (2) it follows that for every component of $V(G) \backslash V(H)$, all its attachments in $H$ are a subset of one of $S_{1}, S_{2}, S_{3}$. Let $X$ be the union of $S_{1}$ and all components of $V(G) \backslash V(H)$ whose attachment set is a subset of $S_{1}$, and let $Y=V(G) \backslash X$. Then $|Y| \geq 4$, and so either $(X, Y)$ is a proper 2-join in $G$, or both $A_{1}, B_{1}$ have one element and $X$ is the vertex set of a path between these two vertices. We may assume the latter, and the same for $S_{2}$ and $S_{3}$; and so $G$ is an even prism. Then either it admits a proper 2-join, or $|V(G)|=9$. This proves 10.6.

\section{Step-connected strips}

Our next target is the statement analogous to 10.6 for long odd prisms, but we need to creep up on it in stages. (A warning: we shall not prove the exact analogue, and we don't know if it is true. We need to permit more types of decomposition, namely proper 2 -joins in $\bar{G}$, and proper homogeneous pairs.) The key idea is to start with a prism of three paths, $R_{0}, R_{1}, R_{2}$, where $R_{0}$ has length $\geq 3$, and to grow the union of the other two paths into a kind of strip (one strip, not two) with a richer internal structure than we have seen hitherto, that we call being "step-connected". If we expand the union of these two paths into a maximal step-connected strip, then the remainder of the graph attaches to this structure in ways that we can exploit. In this section we introduce step-connected strips, and prove some preliminary lemmas about them.

Let $(A, C, B)$ be a strip in $G$. A step is a pair $a_{1}-R_{1}-b_{1}, a_{2}-R_{2}-b_{2}$ of rungs such that

- $V\left(R_{1}\right) \cap V\left(R_{2}\right)=\emptyset$

- $a_{1}$ is adjacent to $a_{2}$, and $b_{1}$ to $b_{2}$, and there are no other edges between $V\left(R_{1}\right)$ and $V\left(R_{2}\right)$.

The edges $a_{1} a_{2}$ and $b_{1} b_{2}$ such that there exists a step as above are called stepped edges. We say that the strip is step-connected if every vertex of $A \cup B \cup C$ is in a step, and for every partition $(X, Y)$ of $A$ or of $B$ into two nonempty sets, there is a step $R_{1}, R_{2}$ such that $R_{1}$ has an end in $X$ and $R_{2}$ has an end in $Y$. (This second condition is equivalent to requiring that the subgraph of $G$ with vertex set $A$ and edges the stepped edges within $A$ be connected, and the same for $B$.) 
Let $(A, C, B)$ be a step-connected strip in a Berge graph $G$. A vertex $v \in V(G) \backslash(A \cup B \cup C)$ is a left-star for the strip if it is complete to $A$ and anticomplete to $B \cup C$, and it is a right-star if it is complete to $B$ and anticomplete to $A \cup C$. A banister (with respect to the strip) is a path $a-R-b$ of $G \backslash(A \cup B \cup C)$, such that $a$ is a left-star, $b$ is a right-star, and there are no edges between the interior of $R$ and $V(S)$. (Here we distinguish between $a-R-b$ and $b-R$ - $a$; we follow the convention that when describing a banister relative to a strip, the end which is the left-star is listed first.) A banister can have length 1 .

11.1 Let $G$ be a Berge graph, such that there is no nondegenerate appearance of $K_{4}$ in $G$. Let $S=$ $(A, C, B)$ be a step-connected strip in $G$, and let $a_{0}-R_{0}-b_{0}$ be a banister. Suppose that $v \in V(G) \backslash V(S)$ has a neighbour in $A \cup C$, and has no neighbour in $B$; and that $P$ is a path in $G \backslash\left(V(S) \cup\left\{a_{0}\right\}\right)$ from $v$ to $b_{0}$, such that there are no edges between $P^{*}$ and $V(S)$. Then $v$ is a left-star.

Proof. Let $F$ be a connected subset of $V(P)$, containing $v$ and disjoint from $V\left(R_{0}\right)$, and with an attachment in $R_{0} \backslash a_{0}$.

(1) For every step $a_{1}-R_{1}-b_{1}, a_{2}-R_{2}-b_{2}$, if $v$ has a neighbour in $R_{1} \cup R_{2}$ then $v$ is adjacent to $a_{1}, a_{2}$ and to no other vertices of $R_{1} \cup R_{2}$.

For assume $v$ has a neighbour in $R_{1}$ say, and hence in $R_{1} \backslash b_{1}$. Now $R_{0}, R_{1}, R_{2}$ form a prism $K$ say, and no vertex in $F$ is major with respect to $K$ since no vertex in $F$ is adjacent to $b_{1}$ or $b_{2}$. Yet $F$ has an attachment in $R_{0} \backslash a_{0}$ and one in $R_{1} \backslash b_{1}$, so its set of attachments is not local. Since $b_{1}$ is not an attachment of $F$, it follows from 10.4 that $F$ has an attachment in $R_{2}$; and therefore $v$ has a neighbour in $R_{2} \backslash b_{2}$. If $v$ has any neighbours in $R_{1} \cup R_{2}$ different from $a_{1}, a_{2}$, say a neighbour in the interior of $R_{1}$, then $v$ can be linked onto the triangle $b_{0}, b_{1}, b_{2}$, via the paths $v-P$ - $b_{0}$, from $v$ to $b_{1}$ with interior in $R_{1} \backslash a_{1}$, and from $v$ to $b_{2}$ with interior in $R_{2}$; but this contradicts 2.4. This proves (1).

From (1) it follows that $v$ has no neighbour in $C$ (since every vertex is in a step), and therefore $v$ has at least one neighbour in $A$; and from (1) again, $v$ has no nonneighbour in $A$ (for otherwise we could choose the step in (1) with $v$ adjacent to $a_{1}$ and not to $a_{2}$, since the strip is step-connected.) This proves 11.1.

11.2 Let $G$ be Berge, such that there is no appearance of $K_{4}$ in $G$. Let $S=(A, C, B)$ be a stepconnected strip in $G$, and let $a_{0}-R_{0}-b_{0}$ be a banister. Let $v \in V(G) \backslash V(S)$ have a neighbour in $V(S)$, and be nonadjacent to $b_{0}$. Let $P$ be a path in $G \backslash\left(V(S) \cup\left\{a_{0}\right\}\right)$ from $v$ to $b_{0}$, and let $Q$ be a path in $G \backslash\left(V(S) \cup\left\{b_{0}\right\}\right)$ from $v$ to $a_{0}$, such there are no edges from $P^{*} \cup Q^{*}$ to $V(S)$. Then either $v$ is $B$-complete, or $v$ is a left-star.

Proof. If $v$ has no neighbours in $B$, then by $11.1 v$ is a left-star, so we may assume $v$ has a neighbour in $B$. Since we may assume it is not $B$-complete, there is a step $a_{1}-R_{1}-b_{1}, a_{2}-R_{2}-b_{2}$ such that $v$ is adjacent to $b_{1}$ and not to $b_{2}$. Let $F \subseteq V(Q)$ be connected, containing $v$ and disjoint from $V\left(R_{0}\right)$, with an attachment in $R_{0} \backslash b_{0}$. Now $R_{0}, R_{1}, R_{2}$ form a prism $K$ say, and no vertex of $F$ is major with respect to $K$ since none of them has two neighbours in $\left\{b_{0}, b_{1}, b_{2}\right\}$. But there is an attachment of $F$ in $R_{0} \backslash b_{0}$, and $b_{1}$ is also an attachment of $F$, so its set of attachments is not local with respect to 
the prism. By 10.1, one of 10.1.1-4 holds. Since there is no appearance of $K_{4}$ in $G, 10.1 .1$ does not hold. Also 10.1.2, 10.1.3 do not hold, since $v$ is the only vertex in $F$ with neighbours in $A \cup B$. So 10.1.4 holds, and therefore $F$ has an attachment in $R_{2}$, and so $v$ has a neighbour in $R_{2}$. But then $v$ can be linked onto the triangle $\left\{b_{0}, b_{1}, b_{2}\right\}$, via $v-P-b_{0}, v-b_{1}$, and the path from $v$ to $b_{2}$ with interior in $R_{2}$, contrary to 2.4. This proves 11.2 .

We remark:

11.3 Let $G$ be Berge, containing no even prism, let $S=(A, C, B)$ be a step-connected strip in $G$, and let $a_{0}-R_{0}-b_{0}$ be a banister. Then every rung of the strip has odd length, and so does $R_{0}$.

Proof. Let $a_{1}-R_{1}-b_{1}, a_{2}-R_{2}-b_{2}$ be a step. Then these three paths form a prism, and it is not an even prism by hypothesis. In particular $R_{0}$ has odd length, by 7.2 . For any rung $a-R$ - $b$, the hole $a_{0}-R_{0}-b_{0}-b-R-a-a_{0}$ has even length, and so $R$ is odd. This proves 11.3.

11.4 Let $G$ be a Berge graph, such that there is no appearance of $K_{4}$ in $G$ and no even prism in $G$. Let $S=(A, C, B)$ be a step-connected strip in $G$. Let $F \subseteq V(G) \backslash(A \cup B \cup C)$ be connected, such that there are no edges between $F$ and $A \cup B \cup C$. There is no anticonnected set $Q \subseteq V(G) \backslash(A \cup B \cup C \cup F)$ such that:

- some right-star has a neighbour in $F$ and a nonneighbour in $Q$,

- some vertex in $B$ has a nonneighbour in $Q$,

- some left-star with a neighbour in $F$ is Q-complete,

- every vertex in $Q$ has a neighbour in $F$,

- every vertex in $Q$ has a neighbour in $A \cup B \cup C$, and

- no vertex in $Q$ is a left-star.

Proof. Suppose that such a set $Q$ exists. Let $a_{0}$ be a left-star with a neighbour in $F$ complete to $Q$, and let $b_{0}$ be a right-star with a neighbour in $F$ and a nonneighbour in $Q$. Let $R_{0}$ be a path between $a_{0}$ and $b_{0}$ with interior in $F$. Hence $a_{0}-R_{0}-b_{0}$ is a banister. By $11.3 R_{0}$ and every rung has odd length. Since some vertex in $B$ has a nonneighbour in $F$, there is an antipath $q_{1}-\cdots-q_{n}$ in $Q$ such that $q_{1}$ is not adjacent to $b_{0}$ and $q_{n}$ is not adjacent to some vertex in $B$. Choose such an antipath with $n$ minimum. Let $B_{1}$ be the set of neighbours of $q_{n}$ in $B$, and $B_{2}=B \backslash B_{1}$. So $B_{2} \neq \emptyset$. Since $q_{n}$ is not a left-star, and there is a path from $q_{n}$ to $b_{0}$ with interior in $F$, it follows from 11.1 that $B_{1} \neq \emptyset$. Choose a step $a_{1}-R_{1}-b_{1}, a_{2}-R_{2}-b_{2}$ with $b_{1} \in B_{1}$ and $b_{2} \in B_{2}$.

(1) $n \geq 2$.

For suppose $n=1$. Then $q_{1}$ is adjacent to $a_{0}$ and to $b_{1}$, and not to $b_{0}$, so by $10.4, q_{1}$ has a neighbour in $R_{2} \backslash b_{2}$. Since $q_{1}$ also has a neighbour in $F$, it can be linked onto the triangle $\left\{b_{0}, b_{1}, b_{2}\right\}$, via a path from $q_{1}$ to $b_{0}$ with interior in $F$, the path $q_{1}-b_{1}$, and the path from $q_{1}$ to $b_{2}$ with interior in $R_{2}$, contrary to 2.4. This proves (1). 
(2) $\left(A \cup B \cup C,\left\{b_{0}, q_{1}, \ldots, q_{n}\right\}\right)$ is balanced.

For $b_{1} \in B_{1}$ is complete to $\left\{b_{0}, q_{1}, \ldots, q_{n}\right\}$ from the minimality of $n$. But $b_{1}$ has no neighbour in $F$, so by $2.6,\left(F,\left\{b_{0}, q_{1}, \ldots, q_{n}\right\}\right)$ is balanced. Since $F$ is connected and every vertex in $\left\{b_{0}, q_{1}, \ldots, q_{n}\right\}$ has a neighbour in $F$, the claim follows from 2.7.1. This proves (2).

Now the path $a_{0}-a_{2}-R_{2}-b_{2}-b_{1}$ is odd, and its ends are complete to $\left\{q_{1}, \ldots, q_{n}\right\}$; so by (2) and 2.1, there are two adjacent vertices $u, v$ in this path, both complete to $\left\{q_{1}, \ldots, q_{n}\right\}$. Since $b_{2}$ is not adjacent to $q_{n}$, it follows that $u, v \in\left\{a_{0}\right\} \cup V\left(R_{2} \backslash b_{2}\right)$. Suppose that the hole $a_{0}-R_{0}-b_{0}-b_{2}-R_{2}-a_{2}-a_{0}$ has length $\geq 6$. Then one of $u, v$ is nonadjacent to both $b_{0}, b_{2}$, say $v$, and hence $n$ is odd, since $v$ - $b_{0}-q_{1}-\cdots-q_{n}-b_{2}-v$ is an antihole; but $b_{1}$ is adjacent to $b_{0}$ and $b_{2}$, and has no other neighbours in this hole, and is complete to $\left\{q_{1}, \ldots, q_{n}\right\}$, contrary to 3.3. So the hole has length 4 , and in particular $a_{2}$ is adjacent to $b_{2}$ and is complete to $\left\{q_{1}, \ldots, q_{n}\right\}$, and $a_{0}$ is adjacent to $b_{0}$. Hence $n$ is odd, because $b_{1}-a_{2}-b_{0}-q_{1}-\cdots-q_{n}-b_{2}-a_{0}-b_{1}$ is an antihole, and so $a_{2}-b_{0}-q_{1^{-}} \cdots-q_{n}-b_{2}$ is an odd antipath, contrary to (2). This proves 11.4 .

A triple $(S, F, Q)$ is called a 1 -breaker in $G$ if it satisfies the following.

- $S=(A, C, B)$ is a step-connected strip in $G$,

- $F \subseteq V(G) \backslash V(S)$ is connected, such that there are no edges between $F$ and $V(S)$, and there is a left- and right-star, both with neighbours in $F$,

- $Q \subseteq V(G) \backslash(V(S) \cup F)$ is anticonnected,

- some vertex in $A$ has a nonneighbour in $Q$, and so does some vertex in $B$,

- every vertex in $Q$ has a neighbour in $F$ and a neighbour in $A \cup B \cup C$,

- some left-star with a neighbour in $F$ is $Q$-complete,

- no vertex in $Q$ is a left-star.

11.5 Let $G$ be a Berge graph, such that there is no appearance of $K_{4}$ in $G$ and no even prism in $G$. If there is a 1-breaker in $G$ then $G$ admits a balanced skew partition.

Proof. Suppose that some 1-breaker $(S, F, Q)$ exists, and for fixed $G$ and $S$, choose $F$ and $Q$ with $|F|+|Q|$ maximum such that all the hypotheses of the theorem remain satisfied (possibly exchanging "left" and "right"). Let $N$ be the set of vertices of $G$ not in $F$ but with a neighbour in $F$. Hence $Q \subseteq N$, and every left- or right-star with a neighbour in $F$ is in $N$. Let $S=(A, C, B)$.

(1) Every vertex in $N$ has a neighbour in $A \cup B \cup C$.

For suppose $v \in V(G) \backslash F$ has a neighbour in $F$ and has none in $A \cup B \cup C$. Let $F^{\prime}=F \cup\{v\}$. Certainly $F^{\prime}$ is connected and disjoint from $A \cup B \cup C$, and there are no edges between $F^{\prime}$ and $A \cup B \cup C$; and $F^{\prime}$ is disjoint from $Q$ since every vertex in $Q$ has a neighbour in $A \cup B \cup C$. It follows that the hypotheses of the theorem remain true, contrary to the maximality of $|F|+|Q|$. This proves (1). 
(2) There is no left- or right-star in $Q$, and every left- and right-star with a neighbour in $F$ is $Q$-complete.

For we are given that there is no left-star in $Q$. Suppose there is a right-star with a neighbour in $F$, either in $Q$ or with a nonneighbour in $Q$. Then there is an antipath with interior in $Q$, between $B$ and some right-star with a neighbour in $F$; but the set of vertices in such an antipath contradicts 11.4. So there is no right-star in $Q$, and every right-star with a neighbour in $F$ is $Q$-complete. We are given that there is a right-star with a neighbour in $F$, and so all hypotheses of the theorem are true with "left" and "right" exchanged. It follows by the same argument, therefore, that every left-star with a neighbour in $F$ is $Q$-complete. This proves (2).

Since $Q \subseteq N$ is anticonnected, it is contained in some anticonnected component of $N$, say $N_{1}$. We may assume that $G$ admits no balanced skew partition, for otherwise the theorem holds.

(3) There is a left- or right-star in $N_{1}$.

For let $N_{2}$ be the union of all the anticomponents of $N$ different from $N_{1}$. Assume that no leftand right-star is in $N_{1}$. Let $Y=V(G) \backslash(F \cup N)$; then there are no edges between $F$ and $Y$, from definition of $N$. Also, $A \cup B \cup C \subseteq Y$, so in particular $Y \neq \emptyset$, and also $N_{2} \neq \emptyset$ since by hypothesis there is a left-star in $N$. Hence $(F \cup Y, N)$ is a skew partition of $G$. By (1), every vertex in $N$ has a neighbour in $A \cup B \cup C$ and in $F$, and so every vertex in $N_{1}$ has a neighbour in $B$ (since otherwise it would be a left-star by 11.1 and therefore belong to $\left.N_{2}\right)$. Now $\left(B \cup C, N_{1}\right)$ is balanced, by 2.6, since any left-star is complete to $N_{1}$ and anticomplete to $B \cup C$. Since $B \cup C$ is connected (because every vertex of $B \cup C$ is in a step and the strip is step-connected), it follows from 2.7.1 that $\left(F, N_{1}\right)$ is balanced. From 4.5, $G$ admits a balanced skew partition, a contradiction. This proves (3).

From (3), $N_{1} \neq Q$; and hence there is a vertex $v \in N \backslash Q$ with a nonneighbour in $Q$. From the maximality of $|F|+|Q|$, replacing $Q$ by $Q \cup\{v\}$ violates one of the hypotheses of the theorem. But $v$ has a neighbour in $A \cup B \cup C$ by (1); $v \notin F$ since it belongs to $N ; v$ is not a left-star since all left-stars in $N$ are $Q$-complete by (2); and so no left-star in $N$ is $Q \cup\{v\}$-complete. Since they are all $Q$-complete, it follows that $v$ is nonadjacent to every left-star in $N$. Similarly $v$ is nonadjacent to every right-star in $N$.

(4) $v$ is complete to $A \cup B$.

For suppose not; then from the symmetry we may assume that $v$ has a nonneighbour in $B$. By $11.2, v$ is a left-star, a contradiction. This proves (4).

Choose an antipath $v-q_{1}-\cdots-q_{k}$ in $Q$, such that $q_{k}$ has a nonneighbour in $A \cup B$, with $k$ minimum. From (4), $k \geq 1$. From the minimality of $k,\left\{v, q_{1}, \ldots, q_{k-1}\right\}$ is complete to $A \cup B$. Let $A_{1}$ be the set of neighbours of $q_{k}$ in $A$, and $A_{2}=A \backslash A_{1}$, and define $B_{1}, B_{2} \subseteq B$ similarly. So $A_{2} \cup B_{2}$ is nonempty.

(5) $k$ is odd. 
For $A_{2} \cup B_{2}$ is nonempty. If there exists $a_{2} \in A_{2}$, let $b_{0} \in N$ be a right-star; then

$$
b_{0}-v-q_{1}-\cdots-q_{k}-a_{2}-b_{0}
$$

is an antihole, so it follows that $k$ is odd. The result follows similarly if $B_{2}$ is nonempty.

(6) $A_{1}$ is complete to $B_{2}$, and $A_{2}$ is complete to $B_{1}$.

For suppose that $a_{1} \in A_{1}$ and $b_{2} \in B_{2}$ are nonadjacent. Let $b_{0} \in N$ be a right-star; then by $(5)$,

$$
b_{0}-v-q_{1}-\cdots-q_{k}-b_{2}-a_{1}-b_{0}
$$

is an odd antihole, a contradiction. So $A_{1}$ is complete to $B_{2}$ and similarly $A_{2}$ is complete to $B_{1}$. This proves (6).

(7) $A_{1}, B_{1}, A_{2}, B_{2}$ are all nonempty.

For we may assume that $A_{2}$ is nonempty. Since the strip is step-connected, every vertex in $A$ has a nonneighbour in $B$, and so by $(6), B_{1} \neq B$. Hence $B_{2}$ is also nonempty. Since $q_{k}$ has a neighbour in $A \cup B \cup C$ it follows that it has a neighbour in $B$, by 11.1, and similarly it has a neighbour in $A$. This proves (7).

Now the strip is step-connected, and so there is a step $a_{1}-R-b_{2}, a_{2}-R^{\prime}-b_{1}$ with $a_{1} \in A_{1}$ and $a_{2} \in A_{2}$. Since $a_{1}$ is not adjacent to $b_{1}$ it follows that $b_{1} \in B_{1}$ by (6), and similarly $b_{2} \in B_{2}$. Also by (6), $R$ and $R^{\prime}$ both have length 1 . Let $a_{0} \in N$ be a left-star and $b_{0} \in N$ a right-star. Since $v-a_{1}-a_{0}-b_{0}-b_{2}-v$ is not an odd hole, it follows that $a_{0}$ is not adjacent to $b_{0}$.

For every vertex $u \in V(G) \backslash F$, let $F_{u}$ be the set of vertices in $F$ adjacent to $u$.

(8) $F_{a_{0}} \cap F_{b_{0}}=\emptyset$, and every path in $F$ between $F_{a_{0}}$ and $F_{b_{0}}$ meets both $F_{v}$ and $F_{q_{k}}$.

For if $f \in F_{a_{0}} \cap F_{b_{0}}$, then $f$ - $a_{0^{-}} a_{1^{-}} b_{2^{-}}-b_{0^{-}} f$ is an odd hole, so $F_{a_{0}} \cap F_{b_{0}}=\emptyset$. Let $p_{1^{-}} P$ - $p_{2}$ be a path in $F$ between $F_{a_{0}}$ and $F_{b_{0}}$, with $V(P)$ minimal, where $p_{1} \in F_{a_{0}}$ and $p_{2} \in F_{b_{0}}$. Hence

$$
a_{0}-p_{1}-P-p_{2}-b_{0}-b_{1}-a_{2}-a_{0}
$$

is a hole, and so $P$ is odd. If $P$ does not meet $F_{v}$ then

$$
v-a_{1}-a_{0}-p_{1}-P-p_{2}-b_{0}-b_{1}-v
$$

is an odd hole, while if $P$ does not meet $F_{q_{k}}$ then

$$
q_{k}-a_{0}-p_{1}-P-p_{2}-b_{0}-q_{k}
$$

is an odd hole, in both cases a contradiction. This proves (8).

(9) Every path in $F$ between $F_{v}$ and $F_{q_{k}}$ meets both $F_{a_{0}}$ and $F_{b_{0}}$. 
For suppose not; then since $F$ is connected and $F_{a_{0}} \cap F_{b_{0}}=\emptyset$, there is a connected subset $F^{\prime}$ of $F$ meeting both $F_{v}, F_{q_{k}}$ and meeting exactly one of $F_{a_{0}}, F_{b_{0}}$. From the symmetry we may assume $F^{\prime}$ meets $F_{a_{0}}$ and not $F_{b_{0}}$. Define $q_{k+1}=a_{2}$; then $q_{k+1}$ has no neighbour in $F^{\prime}$, so we may choose $i$ with $1 \leq i \leq k+1$ minimum such that $q_{i}$ has no neighbour in $F^{\prime}$. Note that $v$ has a neighbour in $F^{\prime}$ (because $F^{\prime}$ meets $F_{v}$ ). If $i$ is even, then $b_{0}-v-q_{1}-\cdots-q_{i}$ is an odd antipath; its internal vertices have neighbours in $F^{\prime}$, and its ends do not, and $a_{1}$ is complete to its interior and has no neighbours in $F^{\prime}$, contrary to 2.2 in the complement. If $i$ is odd, then $b_{1}-a_{0}-v-q_{1}-\cdots-q_{i}$ is an odd antipath, and its internal vertices have neighbours in $F^{\prime}$ and its ends do not, and again $a_{1}$ is complete to its interior and has no neighbours in $F^{\prime}$, contrary to 2.2 in the complement. This proves (9).

Let $f_{1}-f_{2^{-}} \cdots-f_{n}$ be a minimal path in $F$ between $F_{a_{0}}$ and $F_{b_{0}}$, where $f_{1} \in F_{a_{0}}$ and $f_{n} \in F_{b_{0}}$. Then $n \geq 2$ by (8), and by (8) and (9) it follows that $f_{1}-f_{2^{-}} \cdots-f_{n}$ is also a minimal path between $F_{v}$ and $F_{q_{k}}$, so we may assume that $f_{1} \in F_{v}, f_{n} \in F_{q_{k}}$, and no other vertex of the path is in either set. Then $f_{1}-f_{2^{-}} \cdots-f_{n^{-}} q_{k^{-}} a_{0^{-}} f_{1}$ and $f_{1^{-}} f_{2^{-}} \cdots-f_{n^{-}}-b_{0}-b_{1}-v-f_{1}$ are both holes, of different parity, a contradiction. This proves 11.5.

\section{Attachments in a staircase}

For the next step of our approach towards the long odd prism, let us fix a little more than just the strip. Let $S=(A, C, B)$ be a step-connected strip in $G$, and let $a_{0}-R_{0}-b_{0}$ be a banister of length $\geq 3$. We call the pair $K=\left(S, R_{0}\right)$ a staircase, and define $V(K)=V\left(R_{0}\right) \cup V(S)$. (For brevity we often speak of the staircase $K=\left(S=(A, C, B), a_{0}-R_{0}-b_{0}\right)$, meaning that $K=\left(S, R_{0}\right)$ is a staircase, and $S=(A, C, B)$, and $R_{0}$ has ends $a_{0}, b_{0}$, where $a_{0}$ is a left-star and $b_{0}$ is a right-star.) The staircase is maximal if there is no staircase $\left(S^{\prime}=\left(A^{\prime}, C^{\prime}, B^{\prime}\right), a_{0}^{\prime}-R_{0}^{\prime}-b_{0}^{\prime}\right)$ such that $A \subseteq A^{\prime}, B \subseteq B^{\prime}, C \subseteq C^{\prime}$ and $V(S) \subset V\left(S^{\prime}\right)$.

Let $K=\left(S=(A, C, B), a_{0}-R_{0}-b_{0}\right)$ be a staircase in $G$. Some definitions (all with respect to $\left.K\right)$ :

- A subset $X \subseteq V(K)$ is local if $X$ is a subset of one of $V(S), V\left(R_{0}\right), A \cup\left\{a_{0}\right\}, B \cup\left\{b_{0}\right\}$

- $v \in V(G) \backslash V(K)$ is minor if its set of neighbours in $V(K)$ is local

- $v \in V(G) \backslash V(K)$ is major if it has neighbours in all of $A, B$ and $V\left(R_{0}\right)$

- $v \in V(G) \backslash V(K)$ is left-diagonal if $v$ is $\left(A \cup\left\{b_{0}\right\}\right)$-complete, and right-diagonal if it is $\left(B \cup\left\{a_{0}\right\}\right)$ complete

- $v \in V(G) \backslash V(K)$ is central if it is $(A \cup B)$-complete, and is nonadjacent to both $a_{0}$ and $b_{0}$.

First let us examine the possible types of vertices outside the staircase.

12.1 Let $G$ be a Berge graph, such that there is no appearance of $K_{4}$ in $G$, no even prism in $G$, and no 1-breaker in $G$. Let $K=\left(S=(A, C, B), a_{0}-R_{0}-b_{0}\right)$ be a maximal staircase in $G$, and let $v \in V(G) \backslash V(K)$. Then exactly one of the following holds:

1. $v$ is minor; and in that case, either $v$ is a left-star or $v$ is not A-complete, and either $v$ is a right-star or $v$ is not $B$-complete. 
2. $v$ is major; and in that case, it is either left-or right-diagonal or central.

3. $v$ is a left-star with a neighbour in $R_{0} \backslash a_{0}$, or a right-star with a neighbour in $R_{0} \backslash b_{0}$.

\section{Proof.}

(1) If $v$ is left- or right-diagonal then the theorem holds.

For assume $v$ is right-diagonal say. If it has no neighbours in $A \cup C$ then statement 3 of the theorem holds, so we assume there is a step $a_{1}-R_{1}-b_{1}, a_{2}-R_{2}-b_{2}$ such that $v$ has a neighbour in $R_{1} \backslash b_{1}$. Hence it can be linked onto the triangle $\left\{a_{0}, a_{1}, a_{2}\right\}$, via $v$ - $a_{0}$, the path from $v$ to $a_{1}$ with interior in $R_{1} \backslash b_{1}$, and the path from $v$ to $a_{2}$ with interior in $R_{2}$, and so by 2.4,v has a neighbour in $A$. So it is major, and therefore statement 2 holds. This proves (1).

(2) If $v$ is adjacent to both $a_{0}, b_{0}$ then the theorem holds.

For then it has a neighbour in $R_{0}^{*}$, since $R_{0}$ is odd and has length $\geq 3$ and $v$ is adjacent to both its ends; and we may assume that $v$ has a neighbour in $V(S)$, for otherwise statement 1 of the theorem holds. If $v$ has no neighbour in $B$ then it is a left-star by 11.1 , and statement 3 of the theorem holds, so we may assume it has neighbours in $B$ and similarly in $A$. Hence it is major. Since $\left(S, V\left(R_{0}^{*}\right),\{v\}\right)$ is not a 1-breaker, $v$ does not have nonneighbours in both $A$ and $B$, so it is either left- or right-diagonal and the claim follows from (1). This proves (2).

(3) If $v$ is adjacent to $a_{0}$ and not to $b_{0}$ then the theorem holds.

For we may assume $v$ has a neighbour in $V(S)$. If $v$ has a neighbour in $R_{0}^{*}$, then by 11.2 it is either $B$-complete (when it is right-diagonal and the claim follows from (1)) or a left-star (when statement 3 holds). So we may assume it has no neighbour in $R_{0}^{*}$. We may assume it has a neighbour in $B \cup C$, for otherwise it is minor and statement 1 of the theorem holds; let $a_{1}-R_{1}-b_{1}, a_{2}-R_{2}-b_{2}$ be a step such that $v$ has a neighbour in $R_{1} \backslash a_{1}$, and in addition such that $v$ is not adjacent to $b_{2}$ if possible. By 10.4, $v$ has a neighbour in $R_{2}$. If $a_{2}$ is its only neighbour in $R_{2}$, then the strip $S^{\prime}=(A \cup\{v\}, C, B)$ is step-connected, since $v-R-b_{1}, a_{2}-R_{2}-b_{2}$ is an $S^{\prime}$-step where $R$ is the path from $v$ to $b_{1}$ with interior in $R_{1} \backslash a_{1}$; and since $v$ is adjacent to $a_{0}$ and has no other neighbours in $R_{0}$, this is contrary to the maximality of the staircase. So $v$ has a neighbour in $R_{2} \backslash a_{2}$; and hence $v$ can be linked onto the triangle $\left\{b_{0}, b_{1}, b_{2}\right\}$ via $v$ - $a_{0}-R_{0}-b_{0}$, and for $i=1,2$, the path from $v$ to $b_{i}$ with interior in $R_{i} \backslash a_{i}$. By 2.4 it follows that $v$ is adjacent to both $b_{1}, b_{2}$; and hence from our choice of the step $R_{1}, R_{2}$, and since the strip is step-connected, it follows that $v$ is right-diagonal, and the claim follows from (1). This proves (3).

(4) If $v$ is nonadjacent to both $a_{0}, b_{0}$ then the theorem holds.

For then we may assume that $v$ has a neighbour in $V(S)$, since otherwise it is minor, and statement 3 of the theorem holds. Suppose first that $v$ also has a neighbour in $R_{0}^{*}$. If $v$ is a left-star then statement 3 holds, so we assume not; and then by 11.2, $v$ is $B$-complete. Similarly $v$ is $A$-complete and therefore central, and statement 2 holds. Thus we may assume that $v$ has no neighbour in 
$V\left(R_{0}\right)$, and therefore $v$ is minor. We claim that statement 1 holds, and to show this we may assume that $v$ is $A$-complete. Let $a_{1}-R_{1}-b_{1}, a_{2}-R_{2}-b_{2}$ be a step; then by $10.4, v$ has no neighbour in $R_{1} \backslash a_{1}$ or in $R_{2} \backslash a_{2}$, and therefore $v$ is a left-star, and statement 1 holds. This proves (4).

But (2)-(4) cover all the possibilities, up to symmetry, and this completes the proof of 12.1 .

Now let us do the same thing for connected sets.

12.2 Let $G$ be a Berge graph, such that there is no appearance of $K_{4}$ in $G$, no even prism in $G$, and no 1-breaker in $G$. Let $K=\left(S=(A, C, B), a_{0}-R_{0}-b_{0}\right)$ be a maximal staircase in $G$, and let $F \subseteq V(G) \backslash V(K)$ be connected, such that its set of attachments in $V(K)$ is not local with respect to $K$. Then $F$ contains either:

1. a major vertex, or

2. a banister $u-R-v$, such that there are no edges between $V(R)$ and $V\left(R_{0}\right)$, or

3. (up to symmetry) a path $u-R-v$, where $u$ is a left-star, $v$ has a neighbour in $R_{0} \backslash a_{0}$, and there are no edges between $V(R \backslash u)$ and $V(S)$.

Proof. Let $X$ be the set of attachments of $F$ in $V(K)$. We may assume that $F$ is minimal (connected) such that $X$ is not local. Now a subset of $V(K)$ is local if and only if it does not meet both $A \cup C$ and $V\left(R_{0} \backslash a_{0}\right)$ and does not meet both $B \cup C$ and $V\left(R_{0} \backslash b_{0}\right)$; so we may assume that $X$ meets both $A \cup C$ and $V\left(R_{0} \backslash a_{0}\right)$, and therefore from the minimality of $F$, there is a path $f_{1}-\cdots-f_{k}$ where $F=\left\{f_{1}, \ldots, f_{k}\right\}$ and $f_{1}$ is the unique vertex of $F$ with a neighbour in $A \cup C$, and $f_{k}$ is the unique vertex of $F$ with a neighbour in $V\left(R_{0} \backslash a_{0}\right)$. If $k=1$ then the claim follows from 12.1 , so we may assume that $k \geq 2$.

(1) If $f_{1}$ is A-complete then the theorem holds.

For assume $f_{1}$ is $A$-complete. If there is no edge between $F$ and $B \cup C$, then statement 3 of the theorem holds, so we assume that there is such an edge. Choose $i$ with $1 \leq i \leq k$ minimum such that $f_{i}$ has a neighbour in $B \cup C$. Suppose first that there is no edge between $\left\{f_{1}, \ldots, f_{i}\right\}$ and $V\left(R_{0}\right)$. Let $a_{1}-R_{1}-b_{1}, a_{2}-R_{2}-b_{2}$ be a step such that $f_{i}$ has a neighbour in $R_{1} \backslash a_{1}$, and in addition such that $f_{i}$ is nonadjacent to $b_{2}$ if possible. With respect to the prism formed by $R_{0}, R_{1}, R_{2}$, the set of attachments of $\left\{f_{1}, \ldots, f_{i}\right\}$ is not local, and so by 10.4, $i \geq 2$ and its attachments in the prism are $a_{1}, a_{2}, b_{1}, b_{2}$. Hence the only edges between $\left\{f_{1}, \ldots, f_{i}\right\}$ and $V\left(R_{1} \cup R_{2}\right)$ are $f_{1} a_{1}, f_{1} a_{2}, f_{i} b_{1}, f_{i} b_{2}$. From our choice of the step it follows that $f_{i}$ is $B$-complete. Consequently any step satisfies the condition we imposed on $R_{1}, R_{2}$, and so the same conclusion follows for every step; that is, statement 2 of the theorem holds. Now assume that there is an edge between $\left\{f_{1}, \ldots, f_{i}\right\}$ and $V\left(R_{0}\right)$. Suppose that $i<k$; then there is no edge between $\left\{f_{1}, \ldots, f_{i}\right\}$ and $R_{0} \backslash a_{0}$, from the minimality of $F$, and so $a_{0}$ is an attachment of $\left\{f_{1}, \ldots, f_{i}\right\}$. But this set also has an attachment in $B \cup C$, so its set of attachments is not local, contrary to the minimality of $F$. This proves that $i=k$. Since $k \geq 2$, the minimality of $F$ implies that there are no edges between $\left\{f_{2}, \ldots, f_{k}\right\}$ and $V\left(R_{0} \backslash b_{0}\right)$; and so $b_{0}$ is the unique neighbour of $f_{k}$ in $R_{0}$. Hence there are no edges between $\left\{f_{2}, \ldots, f_{k}\right\}$ and $A \cup C$, from the minimality of $F$. Also, there are no edges between $\left\{f_{1}, \ldots, f_{k-1}\right\}$ and $B \cup C$, from the minimality of $i$. Choose a step $a_{1}-R_{1}-b_{1}, a_{2}-R_{2}-b_{2}$ such that $f_{k}$ is adjacent to $b_{1}$, and in addition such that $f_{k}$ is 
nonadjacent to $b_{2}$ if possible. Since $R_{1}$ is odd and $a_{1}-f_{1}-\cdots-f_{k}-b_{1}-R_{1}-a_{1}$ is a hole, it follows that $k$ is even. Since $a_{2}-f_{1^{-}} \ldots-f_{k}-b_{0}-b_{2}-R_{2}-a_{2}$ is not an odd hole, $f_{k}$ is adjacent to $b_{2}$, and therefore to all $B$ from our choice of the step. Since $a_{1^{-}} f_{1^{-}} \cdots-f_{k}-b_{0}-R_{0}-a_{0}-a_{1}$ is not an odd hole and $R_{0}$ is odd, it follows that $f_{1}$ is adjacent to $a_{0}$. But then we can add $f_{1}$ to $A, f_{k}$ to $B$, and $\left\{f_{2}, \ldots, f_{k-1}\right\}$ to $C$, contrary to the maximality of the staircase. This proves (1).

By (1), we may assume there is a step $a_{1}-R_{1}-b_{1}, a_{2}-R_{2}-b_{2}$ such that $f_{1}$ has a neighbour in $R_{1} \backslash b_{1}$, and $a_{2}$ is not adjacent to $f_{1}$. (To see this, first choose a step $a_{1}-R_{1}-b_{1}, a_{2}-R_{2}-b_{2}$ such that $f_{1}$ has a neighbour in $R_{1} \backslash b_{1}$; this satisfies our requirements unless $a_{2}$ is adjacent to $f_{1}$. We may therefore assume that $f_{1}$ has a neighbour and a non-neighbour in $A$; but then since the strip is step-connected, we may choose a step $a_{1}-R_{1}-b_{1}, a_{2}-R_{2}-b_{2}$ so that $f_{1}$ is adjacent to $a_{1}$ and not to $a_{2}$, and again our requirements are satisfied.) Then $R_{0}, R_{1}, R_{2}$ form a prism $K^{\prime}$ say, and the set of attachments of $F$ in $V\left(K^{\prime}\right)$ is not local with respect to $K^{\prime}$. Suppose that some vertex $v$ in $F$ is major with respect to $K^{\prime}$. Then we claim $v$ is major with respect to $K$. For it has a neighbour in $A$ and in $B$, and if it has none in $R_{0}$ then it is adjacent to all of $a_{1}, a_{2}, b_{1}, b_{2}$, in which case $v-a_{1}-a_{0}-R_{0}-b_{0}-b_{2}-v$ is an odd hole. So $v$ is major with respect to $K$, and hence the theorem holds. Hence we may assume that no vertex in $F$ is major with respect to $K^{\prime}$, and so we may apply 10.1. By hypothesis, 10.1.1 does not hold. Since no vertex of $F$ is adjacent to $a_{2}, 10.1 .2$ does not hold.

Suppose that 10.1.3 holds. Since $f_{1}$ is not adjacent to $a_{2}$, it follows that $f_{1}$ is adjacent to $a_{0}, a_{1}$, and there exists $i$ with $2 \leq i \leq k$ such that $f_{i}$ is adjacent to $b_{0}, b_{1}$, and there are no other edges between $\left\{f_{1}, \ldots, f_{i}\right\}$ and $V\left(K^{\prime}\right)$. Then we can add $f_{1}$ to $A, f_{i}$ to $B$ and $\left\{f_{2}, \ldots, f_{i-1}\right\}$ to $C$, contrary to the maximality of the staircase. So 10.1.3 does not hold.

Hence 10.1.4 holds, that is, there is a path $p_{1}-P-p_{2}$ in $F$, such that for some $j$ with $0 \leq j \leq 2$, either:

- $p_{1}$ is adjacent to the two vertices in $\left\{a_{0}, a_{1}, a_{2}\right\} \backslash\left\{a_{j}\right\}$, and $p_{2}$ has neighbours in $R_{j} \backslash a_{j}$, and there are no other edges between $V(P)$ and $V\left(K^{\prime}\right) \backslash\left\{a_{j}\right\}$, or

- $p_{1}$ is adjacent to the two vertices in $\left\{b_{0}, b_{1}, b_{2}\right\} \backslash\left\{b_{j}\right\}$, and $p_{2}$ has neighbours in $R_{j} \backslash b_{j}$, and there are no other edges between $V(P)$ and $V\left(K^{\prime}\right) \backslash\left\{b_{j}\right\}$

From the minimality of $F, F=V(P)$. If $j>0$ then in the first case we can add $p_{1}$ to $A$ and $V\left(P \backslash p_{1}\right)$ to $C$, contrary to the maximality of the staircase; and in the second case we do the same with $A$ and $B$ exchanged. So $j=0$. The first case is impossible since no vertex in $F$ is adjacent to $a_{2}$; and the second case is impossible since $f_{1} \in F=V(P)$ and $f_{1}$ has a neighbour in $R_{1} \backslash b_{1}$. This proves 12.2.

The previous result can be strengthened as follows.

12.3 Let $G$ be a Berge graph, such that there is no appearance of $K_{4}$ in $G$, no even prism in $G$, and no 1-breaker in $G$. Let $K=\left(S=(A, C, B), a_{0}-R_{0}-b_{0}\right)$ be a maximal staircase in $G$, and let $F \subseteq V(G) \backslash V(S)$ be connected, containing a left-star and with an attachment in $B \cup C$. (Note that $F$ may intersect $V\left(R_{0}\right)$.) Then $F$ contains either a major vertex or a banister.

Proof. We may assume $F$ is minimal (possibly exchanging $A$ and $B$ ); so $F$ is the vertex set of a path $f_{1}-\cdots-f_{k}$, where $f_{1}$ is the unique left-star in $F$, and $f_{k}$ is the only vertex in $F$ with a neighbour in $B \cup C$. Since $f_{1}$ is a left-star and $f_{k}$ has a neighbour in $B \cup C$ it follows that $k \geq 2$. We may 
assume there is no major vertex in $F$.

(1) We may assume that none of $f_{1}, \ldots, f_{k}$ is a right-star, and that $f_{k}$ is not B-complete.

For if there is a right-star in $F$, then it must be $f_{k}$; and then from the minimality of $F$ (exchanging $A$ and $B$ ), no vertex of $F$ different from $f_{1}$ has a neighbour in $A \cup C$, and so $f_{1^{-}} \cdots-f_{k}$ is a banister. So we may assume that there is no right-star in $F$. Since $f_{k}$ is neither major nor a right-star, by 12.1 it is not $B$-complete. This proves (1).

(2) $F \cap V\left(R_{0}\right)=\emptyset$, and there are no edges between $\left\{f_{2}, \ldots, f_{k}\right\}$ and $V\left(R_{0} \backslash b_{0}\right)$.

For by $(1), b_{0} \notin F$. Suppose that either $\left\{f_{2}, \ldots, f_{k}\right\}$ intersects $V\left(R_{0} \backslash b_{0}\right)$, or there is an edge joining these two sets. Choose $i$ with $2 \leq i \leq k$ maximum such that either $f_{i} \in V\left(R_{0} \backslash b_{0}\right)$ or $f_{i}$ has a neighbour in $V\left(R_{0} \backslash b_{0}\right)$. We claim that $f_{i} \notin V\left(R_{0}\right)$. For if $i=k$ this is true, since $f_{k}$ has neighbours in $B \cup C$; and if $i<k$ then $f_{i+1}$ has no neighbour in $V\left(R_{0} \backslash b_{0}\right)$ from the maximality of $i$, and therefore again $f_{i} \notin V\left(R_{0}\right)$. So none of $f_{i}, \ldots, f_{k}$ belong to $V\left(R_{0}\right)$. Since $\left\{f_{i}, \ldots, f_{k}\right\}$ has attachments in $V\left(R_{0} \backslash b_{0}\right)$ and in $B \cup C$, and contains no major vertex or left- or right-star, this contradicts 12.2. So $\left\{f_{2}, \ldots, f_{k}\right\}$ is disjoint from $V\left(R_{0} \backslash b_{0}\right)$ and hence from $V\left(R_{0}\right)$, and there are no edges between $\left\{f_{2}, \ldots, f_{k}\right\}$ and $V\left(R_{0} \backslash b_{0}\right)$. Since there is an edge between $\left\{f_{2}, \ldots, f_{k}\right\}$ and $f_{1}$ it follows that $f_{1} \notin V\left(R_{0}\right)$, and so $F \cap V\left(R_{0}\right)=\emptyset$. This proves (2).

Let $a_{1}-R_{1}-b_{1}, a_{2}-R_{2}-b_{2}$ be a step such that $f_{k}$ has a neighbour in $R_{1} \backslash a_{1}$ and $f_{k}$ is nonadjacent to $b_{2}$. (To see that such a step exists, we argue as follows: since $f_{k}$ has a neighbour in $B \cup C$, there is a step $a_{1}-R_{1}-b_{1}, a_{2}-R_{2}-b_{2}$ such that $f_{k}$ has a neighbour in $R_{1} \backslash a_{1}$, and so we may assume that $f_{k}$ is adjacent to $b_{2}$. Hence $f_{k}$ has a neighbour and a nonneighbour in $B$, and the required step exists since the strip is step-connected.)

(3) $f_{1} a_{2}$ is the only edge between $F$ and $R_{2}$.

For if $f_{k}$ has a neighbour in $R_{2}$, then its neighbour set in the prism formed by $R_{0}, R_{1}, R_{2}$ is not local with respect to that prism, and therefore by 10.4, $f_{k}$ has a neighbour in $R_{0}$; and then by 12.1 it is major, a contradiction. So $f_{k}$ has no neighbours in $R_{2}$. From the minimality of $F$, there are no edges between $F$ and $R_{2} \backslash a_{2}$. Suppose that $a_{2}$ has a neighbour in $\left\{f_{2}, \ldots, f_{k}\right\}$, and choose $i$ maximum such that $a_{2}$ is adjacent to $f_{i}$. Since $f_{k}$ has a neighbour in $V\left(R_{1} \backslash a_{1}\right)$, the set of attachments of $\left\{f_{i}, \ldots, f_{k}\right\}$ is not local with respect to the prism formed by $R_{0}, R_{1}, R_{2}$; and since $b_{2}$ is not an attachment, it follows from 10.4 that there is an attachment of $\left\{f_{i}, \ldots, f_{k}\right\}$ in $V\left(R_{0}\right)$. By $(2), b_{0}$ has a neighbour in $\left\{f_{i}, \ldots, f_{k}\right\}$; but then $\left\{f_{i}, \ldots, f_{k}\right\}$ violates 12.2. This proves $(3)$.

(4) $b_{0}$ has neighbours in $\left\{f_{1}, \ldots, f_{k-1}\right\}$.

For first suppose that $b_{0}$ has no neighbour in $F$. Since $b_{2}$ is not an attachment of $F$, it follows from 10.4 (applied to $F$ and the prism formed by $R_{0}, R_{1}, R_{2}$ ) that there is an edge between $F$ and $V\left(R_{0}\right)$, and so $f_{1}$ has a neighbour in $R_{0}$. But then $f_{1}$ can be linked onto the triangle $\left\{b_{0}, b_{1}, b_{2}\right\}$, via the path between $f_{1}$ and $b_{0}$ with interior in $V\left(R_{0}\right)$, the path between $f_{1}$ and $b_{1}$ with interior in $\left\{f_{2}, \ldots, f_{k}\right\} \cup\left(V\left(R_{1}\right) \backslash\left\{a_{1}, b_{1}\right\}\right)$, and the path $f_{1}-a_{2}-R_{2}-b_{2}$. This contradicts 2.4 , and therefore 
proves that $b_{0}$ has a neighbour in $F$. Suppose that $f_{k}$ is the only neighbour of $b_{0}$ in $F$. Then since $f_{k}$ is not major, its unique neighbour in $R_{1}$ is $b_{1}$. From $11.3, R_{1}, R_{2}$ are odd, and from the hole $f_{1}-\cdots-f_{k}-b_{0}-b_{2}-R_{2}-a_{2}-f_{1}$ it follows that $k$ is odd. If $a_{1}$ has no neighbour in $\left\{f_{2}, \ldots, f_{k}\right\}$ then $f_{1}-\cdots-f_{k}-b_{1}-R_{1}-a_{1}-f_{1}$ is an odd hole, and if $a_{1}$ has a neighbour in $\left\{f_{2}, \ldots, f_{k}\right\}$ then $\left\{f_{2}, \ldots, f_{k}\right\}$ violates 12.2. So $f_{k}$ is not the unique neighbour of $b_{0}$ in $F$. This proves (4).

Choose $i$ with $1 \leq i<k$ minimum such that $b_{0}$ is adjacent to $f_{i}$, and let $R_{0}^{\prime}$ be the path $f_{1}-\cdots-f_{i}-b_{0}$. There are no edges between $\left\{f_{1}, \ldots, f_{i}\right\}$ and $B \cup C$ from the minimality of $F$, and from 12.2 there are no edges between $\left\{f_{2}, \ldots, f_{i}, b_{0}\right\}$ and $A \cup C$. Hence $f_{1}-R_{0}^{\prime}-b_{0}$ is a banister, and in particular the three paths $R_{0}^{\prime}, R_{1}, R_{2}$ form a prism, $K^{\prime}$ say. Let $F^{\prime}=\left\{f_{i+1}, \ldots, f_{k}\right\}$. Then $F^{\prime}$ is connected and disjoint from $V\left(K^{\prime}\right)$, and $F^{\prime}$ has attachments in $R_{1} \backslash a_{1}$, and in $R_{0}^{\prime} \backslash b_{0}$, and by (3) it has no attachments in $R_{2}$. By 10.4 applied to $K^{\prime}$, it follows that $F^{\prime}$ contains a path with one end adjacent to $a_{1}, f_{1}$, the other end adjacent to $b_{0}, b_{1}$, and with no more edges between this path and $V\left(R_{0}^{\prime}\right) \cup V\left(R_{1}\right)$. Since the only vertex of $F^{\prime}$ adjacent to $f_{1}$ is $f_{2}$, and that only if $i=1$, and the only vertex in $F^{\prime}$ adjacent to $b_{1}$ is $f_{k}$, it follows that $i=1$, and the only edges between $\left\{f_{2}, \ldots, f_{k}\right\}$ and $V\left(R_{0}^{\prime}\right) \cup V\left(R_{1}\right)$ are $f_{k} b_{1}, f_{k} b_{0}, f_{2} a_{1}, f_{2} f_{1}$. But then by (2), $a_{1}$ can be linked onto the triangle $\left\{b_{0}, b_{1}, f_{k}\right\}$, via $a_{1}-a_{0}-R_{0}-b_{0}, a_{1}-R_{1}-b_{1}, a_{1}-f_{2^{-}} \cdots-f_{k}$, contrary to 2.4 . This proves 12.3 .

Now we turn to anticonnected sets of major vertices. We have already defined what it is for a staircase to be maximal in $G$. We say a staircase $K=\left(S=(A, C, B), a_{0}-R_{0}-b_{0}\right)$ is strongly maximal if it is maximal, and in addition, either $C \neq \emptyset$, or there is no staircase $\left(S^{\prime}, R^{\prime}\right)$ in $\bar{G}$ with $V(S) \subset V\left(S^{\prime}\right)$. A 2-breaker in $G$ is a pair $(K, Q)$ such that

- $K=\left(S=(A, C, B), a_{0}-R_{0}-b_{0}\right)$ is a strongly maximal staircase in $G$,

- $Q \subseteq V(G) \backslash V(K)$ is anticonnected,

- some vertex of $A$ is $Q$-complete, and some vertex of $B$ is $Q$-complete

- $a_{0}, b_{0}$ are not $Q$-complete, and

- some vertex of $R_{0}$ is $Q$-complete.

We observe that if $q$ is a central vertex with respect to a strongly maximal staircase $K$, then $(K,\{q\})$ is a 2-breaker, so it follows from the next result that we no longer have to worry about central vertices.

12.4 Let $G$ be a Berge graph, containing no appearance of $K_{4}$, no even prism, and no 1-breaker. If there is a 2-breaker in $G$ then $G$ admits a balanced skew partition.

Proof. Choose a 2-breaker $(K, Q)$ in $G$, with notation as above, such that for fixed $K$ the set $Q$ is maximal. Let $a_{0}-S$-s and $b_{0}-T-t$ be the subpaths of $R_{0}$ such that $s$ is the unique $Q$-complete vertex of $S$, and $t$ is the unique $Q$-complete vertex of $T$.

(1) $S, T$ both have odd length, and therefore $s, t$ are different.

For choose $a \in A$ and $b \in B$, both $Q$-complete; then $a$ - $a_{0}-S$-s has length $>1$, and its ends are $Q$-complete and its internal vertices are not, and $b$ is also $Q$-complete and has no neighbours in the 
interior of $a-a_{0}-S-s$. By 2.2, this path is even, and so $S$ is odd, and similarly $T$ is odd. Since $R_{0}$ is odd it follows that $s, t$ are different. This proves (1).

(2) Every vertex in $A \cup B$ is $Q$-complete.

For suppose some vertex in $A$ say is not $Q$-complete. Choose a step $a_{1}-R_{1}-b_{1}, a_{2}-R_{2}-b_{2}$ such that $a_{1}$ is $Q$-complete and $a_{2}$ is not. Since $s, t$ are different it follows that $t$ is nonadjacent to both $a_{0}, a_{2}$; and so by 2.8, $Q$ cannot be linked onto the triangle $\left\{a_{0}, a_{1}, a_{2}\right\}$. Hence there is no $Q$-complete vertex in $R_{2}$. Assume $s, t$ are nonadjacent; then the subpath of $R_{0}$ between them is odd, and $a_{1}$ has no neighbour in its interior, so by 2.2 it contains another $Q$-complete vertex $u$ say; and then $s-S-a_{0}-a_{2}-R_{2}-b_{2}-b_{0}-T-t$ is an odd path, its ends are $Q$-complete and its internal vertices are not, and $u$ has no neighbour in its interior, contrary to 2.2. So $s, t$ are adjacent. Hence the hole $a_{0}-R_{0}-b_{0}-b_{2}-R_{2}-a_{2}-a_{0}$ has length $\geq 6$, and the only $Q$-complete vertices in it are the adjacent vertices $s, t$. By $2.10 Q$ contains a hat or a leap; and in either case there is a vertex $q \in Q$ with no neighbours in $R_{2}$. But $q$ is adjacent to $s$ and $a_{1}$, contrary to 10.4 applied to the prism formed by $R_{0}, R_{1}, R_{2}$. This proves (2).

(3) Every major vertex is either in $Q$ or complete to $Q$.

For let $v$ be a major vertex, and suppose $v \notin Q$, and $Q^{\prime}$ is anticonnected, where $Q^{\prime}=Q \cup\{v\}$. From 12.1, $v$ is either left- or right-diagonal, or central; and in either case it has neighbours $a_{1} \in A$ and $b_{1} \in B$ that are nonadjacent. It follows that $a_{1}-a_{0}-R_{0}-b_{0}-b_{1}$ is an odd path of length $\geq 5$, and its ends are $Q^{\prime}$-complete. From the maximality of $Q$, none of its internal vertices are $Q^{\prime}$-complete, and so by 2.1, $Q^{\prime}$ contains a leap $q_{1}, q_{2}$ say. So neither of $q_{1}, q_{2}$ has neighbours in the interior of $R_{0}$; but this is impossible since one of them is in $Q$ and is therefore adjacent to $s$. This proves (3).

(4) There is no edge uv of $G \backslash V(S)$ such that $u$ is a left-star, $v$ is a right-star, and $u$, $v$ are not $Q$-complete.

For suppose $u v$ is such an edge. Since $u, v$ have neighbours in $A \cup B$, they do not belong to $R_{0}^{*}$. Since $u, v$ have nonneighbours in $Q$ and $Q$ is anticonnected, there is an antipath $u-q_{1}-\cdots-q_{k}-v$ with $q_{1}, \ldots, q_{k} \in Q$. Choose a step $a_{1}-R_{1}-b_{1}, a_{2}-R_{2}-b_{2}$. Then $a_{1}-b_{2}-u-q_{1}-\cdots-q_{k}-v-a_{1}$ is an antihole, so $k$ is even. Hence every $Q$-complete vertex $w$ say is adjacent to one of $u$, $v$, for otherwise $w-u-q_{1^{-}} \cdots-q_{k}-v-w$ would be an odd antihole. In particular, there are no $Q$-complete vertices in $C$; and therefore $a_{1}-R_{1}-b_{1}$ is an odd path with both ends $Q$-complete and no internal vertex $Q$-complete. Since $a_{2}$ is $Q$-complete and has no neighbour in the interior of $R_{1}$, it follows from 2.2 that $R_{1}$ has length 1 , and similarly $R_{2}$ has length 1 . Since this step was arbitrary, and every vertex is in a step, it follows that $C=\emptyset$. Suppose that $u$ has no neighbour in $R_{0}^{*}$. Then all $Q$-complete vertices in $R_{0}^{*}$ are adjacent to $v$. In particular, $v$ is adjacent to $s, t$ and hence does not belong to $R_{0}$ (because $v$ is a right-star); and $s-S-a_{0}-a_{1}-b_{1}$ is an odd path, its ends are $(Q \cup\{v\})$-complete, its internal vertices are not, and the $(Q \cup\{v\})$-complete $t$ has no neighbour in its interior, contrary to 2.2. So $u$ has a neighbour in $R_{0}^{*}$, and similarly so does $v$. Now $b_{1}-u-Q-v-a_{1}$ is an odd antipath, all its internal vertices have neighbours in the connected set $R_{0}^{*}$, and its ends do not. By 2.1 applied in $\bar{G}$, there is a leap; that is, there exist adjacent $a, b \in R_{0}^{*}$, both $Q$-complete, such that $b-u-Q-v-a$ is an antipath. Define $A^{\prime}=A \cup\{a\}$ and $B^{\prime}=B \cup\{b\}$; then $\left(A^{\prime}, \emptyset, B^{\prime}\right)$ is a strip ( $S^{\prime}$ say) in $\bar{G}$. For every edge $a_{1} b_{1}$ of $G$ with $a_{1} \in A$ and 
$b_{1} \in B$, the pair $a-b_{1}, a_{1}-b$ is a step of $S^{\prime}$ (in $\bar{G}$ ), and every vertex of $A \cup B$ is in such an edge, and so $S^{\prime}$ is step-connected. Hence $\left(\left(A^{\prime}, \emptyset, B^{\prime}\right), v-q_{k^{-}} \cdots-q_{1}-u\right)$ is a staircase in $\bar{G}$, contrary to the hypothesis that $K$ is strongly maximal. This proves (4).

(5) Every path in $G$ from an A-complete vertex to a vertex with a neighbour in $B \cup C$ contains either a vertex in $Q$ or a $Q$-complete vertex.

For suppose not, and choose a path $p_{1}-\cdots-p_{k}$ say, with $k$ minimum such that $p_{1}$ is $A$-complete and $p_{k}$ has a neighbour in $B \cup C$, and none of $p_{1}, \ldots, p_{k}$ is in $Q$ or $Q$-complete. Since $A \cup B$ is complete to $Q$ it follows that none of $p_{1}, \ldots, p_{k}$ is in $A \cup B$. Now $p_{1}$ is not in $C$ since no vertex in $C$ is $A$-complete (because they are all in steps), and if some $p_{i} \in C$ for $i>1$, then $p_{1}-\cdots-p_{i-1}$ is a shorter path with the same properties, contrary to the minimality of $k$. So none of $p_{1}, \ldots, p_{k}$ is in $V(S)$. (Some may be in $R_{0}$, however.) Since none of $p_{1}, \ldots, p_{k}$ is major by (3), it follows from 12.3 and the minimality of $k$ that $p_{1}-\cdots-p_{k}$ is a banister. From (4), since none of $p_{1}, \ldots, p_{k}$ is $Q$-complete, it follows that $k>2$. Let $a_{1}-R_{1}-b_{1}, a_{2}-R_{2}-b_{2}$ be a step. From the hole $a_{1}-p_{1}-\cdots-p_{k}-b_{1}-R_{1}-a_{1}$ it follows that $k$ is even; and so $a_{1}-p_{1}-\cdots-p_{k}-b_{2}$ is an odd path of length $\geq 5$; its ends are $Q$-complete, and its internal vertices are not. By $2.1, Q$ contains a leap $a, b$; so $a-p_{1}-\cdots-p_{k}-b$ is a path. But then $(A \cup\{a\}, C, B \cup\{b\})$ is a step-connected strip $S^{\prime}$ say (since for every nonadjacent $a^{\prime} \in A$ and $b^{\prime} \in B$, the two paths $a-b^{\prime}, a^{\prime}-b$ make a step in this strip), and so $\left(S^{\prime}, p_{1^{-}} \cdots-p_{k}\right)$ is a staircase, contrary to the maximality of $\left(S, R_{0}\right)$. This proves $(5)$.

Let $X$ be the set of all $Q$-complete vertices in $G$; let $M$ be the component of $G \backslash(Q \cup X)$ that contains $a_{0}$, and $N$ the union of all the other components. By (5), $b_{0} \in N$, so $N$ is nonempty, and hence $(M \cup N, Q \cup X)$ is a skew partition of $G$. Choose $b \in B$; then $b \in X$, and it has no neighbour in $M$ by (5). Hence the skew partition is loose, and so $G$ admits a balanced skew partition, by 4.2. This proves 12.4 .

12.5 Let $G$ be a Berge graph, containing no appearance of $K_{4}$, no even prism, no 1-breaker and no 2-breaker. Let $K=\left(S=(A, C, B), a_{0}-R_{0}-b_{0}\right)$ be a strongly maximal staircase in $G$. Let $q_{1}-\cdots-q_{k}$ be an antipath such that $q_{2}, \ldots, q_{k-1}$ are both left- and right-diagonal, and $q_{1}$ is left- and not rightdiagonal, and $q_{k}$ is right- and not left-diagonal. Then $q_{1}$ is a left-star and $q_{k}$ is a right-star.

Proof. First, obviously $k \geq 2$. Let $Q=\left\{q_{1}, \ldots, q_{k}\right\}$.

(1) If $q_{1}$ is adjacent to $a_{0}$ and $q_{k}$ to $b_{0}$ then the theorem holds.

For then both $a_{0}, b_{0}$ are $Q$-complete, and $q_{1}$ has a nonneighbour in $B$ (for otherwise it would be right-diagonal), and $q_{k}$ has a nonneighbour in $A$. Since $R_{0}$ has odd length $\geq 3$, it follows that each of $q_{1}, \ldots, q_{k}$ has a neighbour in $R_{0}^{*}$. Since $\left(S, R_{0}^{*}, Q\right)$ is not a 1-breaker, it follows that $Q$ contains a left-star, which must be $q_{1}$; and similarly $q_{k}$ is a right-star. Then the theorem holds. This proves (1).

(2) If $q_{1}$ is adjacent to $a_{0}$ and $q_{k}$ is nonadjacent to $b_{0}$ then the theorem holds.

For in this case, $q_{1}$ has a nonneighbour in $B$, say $b$. From the antihole $a_{0}-b-q_{1}-\cdots-q_{k}-b_{0}-a_{0}$ we deduce that $k$ is odd. Now $R_{0}$ is odd, of length $\geq 3$, and its ends are complete to $Q \backslash\left\{q_{k}\right\}$, and so is 
every $a \in A$, and $a$ has no neighbour in the interior of $R_{0}$, so by 2.2 , there is a $\left(Q \backslash\left\{q_{k}\right\}\right)$-complete vertex in the interior of $R_{0}$, say $t$. Let $T$ be the subpath of $t$ to $b_{0}$, and let us choose $t$ with $T$ of minimum length, that is, such that $t$ is the unique $\left(Q \backslash\left\{q_{k}\right\}\right)$-complete vertex of $T$. If $t$ is nonadjacent to $q_{k}$ then $t-b-q_{1}-\cdots-q_{k}$ - $t$ is an odd antihole (since $k \geq 2$ ), a contradiction. Hence $t$ is $Q$-complete, and in particular, all of $q_{1}, \ldots, q_{k}$ have neighbours in the interior of $R_{0}$. By 11.4 it follows that $Q$ contains a left-star, which must be $q_{1}$. We may assume that $q_{k}$ is not a right-star, for otherwise the theorem holds. Since $q_{k}$ is right-diagonal, from 12.1 it follows that $q_{k}$ is major and therefore has a neighbour in $A$. Choose a step $a_{1}-R_{1}-b_{1}, a_{2}-R_{2}-b_{2}$ such that $q_{k}$ is adjacent to $a_{1}$, and if possible nonadjacent to $a_{2}$. Then $t-T-b_{0}-b_{1}-R_{1}-a_{1}$ is a path, and both its ends are $Q$-complete, and none of its internal vertices are $Q$-complete (since $q_{1}$ is a left-star). By 3.2 applied to $t-T-b_{0}-b_{1}-R_{1}-a_{1}$ and the antipath $b_{1}-q_{1}-\cdots-q_{k}-b_{0}$, it follows that $t-T-b_{0}-b_{1}-R_{1}-a_{1}$ has length 4 , and so $R_{1}$ has length 1 and $T$ has length 2; let its middle vertex be $u$ say. Also from 3.2, $u$ is $Q \backslash\left\{q_{1}\right\}$-complete, and nonadjacent to $q_{1}$. Suppose that $q_{k}$ is nonadjacent to $a_{2}$. Then there is no $Q$-complete vertex in $R_{2}$. If $t$ is nonadjacent to $a_{0}$ then $a_{0}-a_{2}-R_{2}-b_{2}-b_{0}-u-t$ is an odd path of length $\geq 5$; its ends are $Q$-complete and its internal vertices are not, so by 2.1, $Q$ contains a leap, which is impossible since every vertex in $Q$ is adjacent to one of $b_{0}, b_{2}$. If $t$ is adjacent to $a_{0}$, then $a_{0}-a_{2}-R_{2}-b_{2}-b_{0}-R_{0}-a_{0}$ is a hole of length $\geq 6$, and the only $Q$-complete vertices in it are $a_{0}, t$, and these are adjacent; so by 2.10 there is a hat or a leap in $Q$; and again this is impossible since every vertex in $Q$ is adjacent to one of $b_{0}, b_{2}$. This proves that $q_{k} a_{2}$ is an edge. From our choice of the step, it follows that $q_{k}$ is $A$-complete. But therefore any step satisfies the condition we imposed on the step $R_{1}, R_{2}$; and therefore every path in every step has length 1 , that is $C=\emptyset$. Then $S=(A \cup\{t\}, \emptyset, B \cup\{u\})$ is a step-connected strip in $\bar{G}$, and $\left(S^{\prime}, b_{0}-q_{k^{-}} \cdots-q_{1}\right)$ is a staircase in $\bar{G}$, contradicting that $\left(S, R_{0}\right)$ is strongly maximal. This proves (2).

(3) If $q_{1}$ is nonadjacent to $a_{0}$ and $q_{k}$ is nonadjacent to $b_{0}$ then the theorem holds.

For then $a_{0}-q_{1}-\cdots-q_{k}-b_{0}-a_{0}$ is an antihole, so $k$ is even. Let $A_{1}$ be the set of vertices in $A$ adjacent to $q_{k}$, and $A_{2}=A \backslash A_{1}$; and let $B_{1}$ be the set of vertices in $B$ adjacent to $q_{1}$, and $B_{2}=B \backslash B_{1}$. If $a_{1} \in A_{1}$ and $b_{2} \in B_{2}$, then $a_{1}-b_{2}-q_{1}-\cdots-q_{k}-b_{0}-a_{1}$ is not an odd antihole, and so $a_{1}$ is adjacent to $b_{2}$; and hence $A_{1}$ is complete to $B_{2}$, and similarly $A_{2}$ is complete to $B_{1}$. If $A_{1}, B_{1}$ are both empty then by 12.1 , the theorem holds; so we may assume that $A_{1}$ is nonempty. Choose $a_{1} \in A_{1}$. Since $a_{1}$ is in a step, it has a nonneighbour in $B$, say $b_{1}$. Since $a_{1}$ is $B_{2}$-complete it follows that $b_{1} \in B_{1}$. Then $a_{1}, b_{1}$ are both $Q$-complete, and since $(K, Q)$ is not a 2-breaker, no internal vertex of $R_{0}$ is $Q$-complete. So $a_{1}-a_{0}-R_{0}-b_{0}-b_{1}$ is an odd path of length $\geq 5$, and its ends are $Q$-complete, and its internal vertices are not. By 2.1, $Q$ contains a leap. Since every vertex of $Q$ except $q_{1}, q_{k}$ has $\geq 2$ neighbours in $R_{0}$, it follows that $k=2$ and $q_{1}, q_{2}$ both have no neighbours in the interior of $R_{0}$. Then $S^{\prime}=\left(A \cup\left\{q_{2}\right\}, C, B \cup\left\{q_{1}\right\}\right)$ is a step-connected strip (since $a_{1}-q_{1}, q_{2}-b_{1}$ is a step of it), and $\left(S^{\prime}, R_{0}\right)$ is a staircase, contrary to the maximality of $\left(S, R_{0}\right)$. This proves $(3)$.

From (1),(2),(3), the theorem follows. This proves 12.5. 


\section{The long odd prism}

In this section we apply the results of the previous section to prove that a Berge graph containing a long odd prism has a decomposition unless it is a line graph.

Let $K=\left((A, C, B), a_{0}-R_{0}-b_{0}\right)$ be a strongly maximal staircase in a Berge graph $G$. From 12.1 there are three possible kinds of $B$-complete vertices; right-stars, vertices complete to both $A$ and $B$, and $B$-complete vertices adjacent to some but not all of $A$. The most difficult step in handling the long odd prism is when there is a vertex of the third kind. In that case, we shall construct a subset of $B$-complete vertices, including all these "mixed" vertices and some of the others, such that they and their common neighbours form a cutset of the graph, and thereby give us a skew partition. We define the set recursively as follows: initially let $X$ be the set of all $B$-complete vertices adjacent to some but not all of $A$. Then enlarge $X$ by repeatedly applying the following two rules, in any order:

1. if there is an $A \cup B$-complete vertex $v$ that is not in $X$ and not $X$-complete, add $v$ to $X$

2. if there is a banister $a-R-b$ such that $a$ is not $X$-complete and $b$ is not in $X$, add $b$ to $X$.

The process eventually stops with some set $X$. We shall prove that $X$ and its common neighbours (say $Y$ ) separate $A$ (or at least the part of $A$ that is not $X$-complete) from $b_{0}$, and this will provide a balanced skew partition. To prove that $X \cup Y$ separates $G$ as described, we have to show that every path from $A$ to $b_{0}$ meets $X \cup Y$, and it turns out that there are only two kinds of paths to worry about; banisters, and 1-vertex paths consisting of a major vertex. Any banister $a-R-b$ is automatically hit, because of the rule above; if $a \notin Y$ then $b \in X$. The 1-vertex paths are trickier. Let $v$ be a major vertex. If it is $B$-complete, then it is in either $Y$ or $X$ by the rule above, so assume it is not $B$-complete. By 12.1, it is left- and not right-diagonal, and now we have to show it belongs to $Y$. If only we knew that every vertex in $X$ was adjacent to $a_{0}$, then it follows easily that $v \in Y$, because of 12.5. So that is what we need to do - to prove that every vertex in $X$ is adjacent to $a_{0}$.

Let us start again, more formally. Let $K=\left((A, C, B), a_{0}-R_{0}-b_{0}\right)$ be a staircase in a Berge graph $G$. We define a right-sequence to be a sequence $x_{1}, \ldots, x_{t}$, with the following properties (which we refer to as the right-sequence axioms):

1. $x_{1}, \ldots, x_{t}$ are distinct and $B$-complete

2. for $1 \leq i \leq t$, if $x_{i}$ is $A$-complete then there exists $h$ with $1 \leq h<i$ such that $x_{h}$ is nonadjacent to $x_{i}$

3. for $1 \leq i \leq t$, if $x_{i}$ is $A$-anticomplete then there is a banister $r$ - $R$ - $x_{i}$ such that $r$ has a nonneighbour in $\left\{x_{1}, \ldots, x_{i-1}\right\}$.

Any initial subsequence of a right-sequence is therefore another right-sequence. We say $x_{i}$ is earlier than $x_{j}$ if $i<j$. Let $X=\left\{x_{1}, \ldots, x_{t}\right\}$. For each $x_{i} \in X$ that has an earlier nonneighbour, we define its predecessor to be $x_{h}$, where $h$ is minimum such that $1 \leq h<i$ and $x_{h}$ is nonadjacent to $x_{i}$. From the second axiom, every $x_{i}$ either has a nonneighbour in $A$ or a predecessor, so we can follow the sequence of predecessors until we get to some vertex that is not $A$-complete. For each $x_{i}$ we therefore define the trajectory of $x_{i}$ to be the sequence $w_{1}-\cdots-w_{n}$ with the following properties:

- $n \geq 1$, and $w_{1}=x_{i}$ 
- $w_{n}$ has a nonneighbour in $A$

- for $1 \leq j<n, w_{j}$ is $A$-complete, and $w_{j+1}$ is the predecessor of $w_{j}$.

Clearly the trajectory is unique, and is an antipath. If $v \in V(G)$ is $A$-complete, not in $X$ and not $X$-complete, we define the trajectory of $v$ to be the antipath $v-w_{1}-\cdots-w_{n}$, where $w_{1}$ is the earliest nonneighbour of $v$ in $X$, and $w_{1}-\cdots-w_{n}$ is the trajectory of $w_{1}$.

Let $a$ be a left-star. If it is not $X$-complete, we define the birth of $a$ to be the earliest nonneighbour of $a$ in $X$. Now let $b$ be a right-star. A banister $a-R$ - $b$ is said to be $b$-optimal if $a$ is not $X$-complete, and there is no banister $a^{\prime}-R^{\prime}-b$ such that $a^{\prime}$ is not $X$-complete and the birth of $a^{\prime}$ is earlier than the birth of $a$.

13.1 Let $G$ be Berge, containing no appearance of $K_{4}$, no even prism, no 1-breaker and no 2breaker. Let $K=\left(S=(A, C, B), a_{0}-R_{0}-b_{0}\right)$ be a strongly maximal staircase in $G$, and let $x_{1}, \ldots, x_{t}$ be a right-sequence. Let $b$ be a right-star, and let $a-R$-b be a b-optimal banister. Let $a-w_{1}-\cdots-w_{n}$ be the trajectory of $a$. Then $n$ is odd, and either:

- $b$ is the unique vertex of $R$ which is $\left\{w_{1}, \ldots, w_{n}\right\}$-complete, or

- $R$ has length 1 , and there exists some even $m$ with $1 \leq m<n$ such that $a-w_{1}-\cdots-w_{m}-b$ is an antipath.

Proof. We proceed by induction on $t$, and assume the result holds for all smaller values of $t$. Hence we may assume that $w_{1}=x_{t}$, for otherwise the result follows from the inductive hypothesis. Let $W$ $=\left\{w_{1}, \ldots, w_{n}\right\}$; then every vertex in $B$ is $W$-complete.

(1) $n$ is odd.

For choose $a_{2} \in A$ nonadjacent to $w_{n}$, and $b_{1} \in B$ nonadjacent to $a_{2}$; then $b_{1}-a-w_{1}-\cdots-w_{n}-a_{2}-b_{1}$ is an antihole, so $n$ is odd. This proves (1).

(2) If $w_{n}$ has a neighbour in $A$ then the theorem holds.

For choose a step $a_{1}-R_{1}-b_{1}, a_{2}-R_{2}-b_{2}$ such that $w_{n}$ is adjacent to $a_{1}$ and not to $a_{2}$. Then $a_{1}, b_{2}$ are $W$-complete. Suppose first that there are no $W$-complete vertices in $R$. Then $a_{1}-a-R-b-b_{2}$ is an odd path between $W$-complete vertices. If $R$ has length 1 then there is an antipath $Q$ joining $a, b$ with interior in $W$, and since it can be completed to an antihole via $b-a_{1}-b_{2}-a$, it has odd length and the theorem holds. So we may assume $R$ has length $>1$, and hence by $2.1 W$ contains a leap. Since all vertices of $W$ except $w_{1}$ are adjacent to $a$, the leap is $w_{1}, w_{2}$; and hence the only edges between $w_{1}, w_{2}$ and $R$ are $w_{1} b$ and $w_{2} a$. Since $n$ is odd it follows that $n>2$ and so $w_{1}, w_{2}$ are both $A \cup B$-complete. But then $S^{\prime}=\left(A \cup\left\{w_{2}\right\}, C, B \cup\left\{w_{1}\right\}\right)$ is a step-connected strip, and $\left(S^{\prime}, a-R-b\right)$ is a staircase, contrary to the maximality of $\left(S, R_{0}\right)$. So we may assume there are $W$-complete vertices in $R$. If $b$ is the only one then the theorem holds, so assume there is another. But then $W$ can be linked onto the triangle $\left\{a, a_{1}, a_{2}\right\}$, via a subpath of $R \backslash b$, the 1 -vertex path $a_{1}$, and a subpath of $R_{2}$. Since $b_{1}$ is $W$-complete and nonadjacent to both $a, a_{2}$, this contradicts 2.8. This proves (2).

From (2) we may assume that $w_{n}$ has no neighbour in $A$. Let $w_{n}=x_{s}$ say. From the third axiom, there is a banister $r^{\prime}-R^{\prime}-w_{n}$, such that $r^{\prime}$ has a nonneighbour in $\left\{x_{1}, \ldots, x_{s-1}\right\}$, and therefore 
we may choose this banister to be $w_{n}$-optimal.

(3) $R^{\prime}$ is disjoint from $R$, and there are no edges between $V(R \backslash a)$ and $V\left(R^{\prime} \backslash w_{n}\right)$.

Suppose that $(R \backslash a) \cup\left(R^{\prime} \backslash w_{n}\right)$ is connected. Then it contains a path between $r^{\prime}$ and $b$, with interior in the union of the interiors of $R$ and $R^{\prime}$, and therefore this path is a banister. But $R$ is $b$-optimal, and the birth of $r^{\prime}$ is earlier than the birth of $a$, a contradiction. So $R \backslash a$ is disjoint from $R^{\prime} \backslash w_{n}$, and there are no edges between them. Since $a \neq r^{\prime}$ (because their births are different), and $b \neq w_{n}$ (because $R$ is optimal for $b$ ) it follows that $R$ is disjoint from $R^{\prime}$. This proves (3).

Let $r^{\prime}-v_{1}-\cdots-v_{m}$ be the trajectory of $r^{\prime}$, and let $V=\left\{v_{1}, \ldots, v_{m}\right\}$. By the inductive hypothesis, it follows that either $w_{n}$ is the unique $V$-complete vertex in $R^{\prime}$, or $R^{\prime}$ has length 1 and there is an odd antipath between $r^{\prime}$ and $w_{n}$ with interior in $V$. Since each of $v_{1}, \ldots, v_{m}$ is earlier than $w_{n}$, it follows from the definition of trajectory that $v_{1}, \ldots, v_{m}$ are all $\left\{a, w_{1}, \ldots, w_{n-1}\right\}$-complete.

(4) If $n=1$ then the theorem holds.

For let $n=1$, and choose a step $a_{1}-R_{1}-b_{1}, a_{2}-R_{2}-b_{2}$ with $a_{1}$ nonadjacent to $v_{m}$. Suppose first that $a$ has no neighbour in $R^{\prime}$. Now $a$ is $V$-complete, and either $w_{1}$ is the unique $V$-complete vertex in $R^{\prime}$, or $R^{\prime}$ has length 1 and there is an odd antipath $Q$ between $r^{\prime}$ and $w_{1}$ with interior in $V$. In the first case, $a-a_{1}-r^{\prime}-R^{\prime}-w_{1}$ is an odd path, its ends are $V$-complete, its internal vertices are not, and the $V$-complete vertex $b_{2}$ has no neighbour in its interior, contrary to 2.2 . In the second case, $a-r^{\prime}-Q-w_{1}-a$ is an odd antihole. This proves that $a$ has a neighbour in $R^{\prime}$. Now suppose it has a neighbour different from $r^{\prime}$; then $R^{\prime}$ has length $>1$, and so $w_{1}$ is the unique $V$-complete vertex in $R^{\prime}$; and there is a path $P^{\prime}$ say from $a$ to $w_{1}$ with interior in $R^{\prime} \backslash r^{\prime}$. Since the ends of this path are $V$-complete and its internal vertices are not, and the $V$-complete vertex $b_{1}$ has no neighbour in its interior, it is even by 2.2. But it can be completed to an odd hole via $w_{1}-b_{1}-R_{1}-a_{1}-a$, a contradiction. This proves that $r^{\prime}$ is the unique neighbour of $a$ in $R^{\prime}$. Since $a-r^{\prime}-R^{\prime}-w_{1}-b_{1}-b-R-a$ is not an odd hole, it follows from (3) that $w_{1}$ has a neighbour in $R$. If $b$ is its unique neighbour in $R$ then the theorem holds, so we assume not. Then there is a path $P$ say from $w_{1}$ to $a$ with interior in $R \backslash b$. Since $w_{1}-P-a-r^{\prime}-R^{\prime}-w_{1}$ is a hole it follows that $P$ is even; but $P$ can be completed via $a-a_{1}-R_{1}-b_{1}-w_{1}$, a contradiction. This proves (4).

We may therefore assume that $n \geq 3$ (since it is odd.)

(5) $C=\emptyset$.

For suppose not, and choose a step $a_{1}-R_{1}-b_{1}, a_{2}-R_{2}-b_{2}$ where $R_{1}$ has length $>1$. Since $R_{1}$ is odd, and its ends are $\left(W \backslash\left\{w_{n}\right\}\right)$-complete, and the $\left(W \backslash\left\{w_{n}\right\}\right)$-complete vertex $b_{2}$ has no neighbour in its interior, there is a $\left(W \backslash\left\{w_{n}\right\}\right)$-complete vertex $v$ in the interior of $R_{1}$, by 2.2 . But then $v$ is nonadjacent to both $a$ and $w_{n}$, since they are left- and right-stars respectively, and so $v-a-w_{1}-\cdots-w_{n}-v$ is an odd antihole, a contradiction. This proves (5).

(6) If $b$ is not $\left(W \backslash\left\{w_{n}\right\}\right)$-complete and no edge of $R$ is $\left(W \backslash\left\{w_{n}\right\}\right)$-complete then the theorem holds. 
For choose a step $a_{1}-R_{1}-b_{1}, a_{2}-R_{2}-b_{2}$. Then $a_{1}-a-R-b-b_{2}$ is an odd path, its ends are $\left(W \backslash\left\{w_{n}\right\}\right)$ complete, and none of its edges are $\left(W \backslash\left\{w_{n}\right\}\right)$-complete. Suppose first that $R$ has length $\geq 3$. Then by 2.1 there is a leap in $W \backslash\left\{w_{n}\right\}$; and so there are nonadjacent vertices $x, y \in W \backslash\left\{w_{n}\right\}$ such that $x$ - $a-R-b-y$ is a path. But then $((A \cup\{x\}, \emptyset, B \cup\{y\}), a-R-b)$ is a staircase, contrary to the maximality of $\left(S, R_{0}\right)$. So $R$ has length 1 , and there exists $i$ with $1 \leq i<n$ such that $a-w_{1}-\cdots-w_{i}-b$ is an odd antipath. But then the theorem holds. This proves (6).

(7) If no vertex in $R$ is $W$-complete then the theorem holds.

For by (6) we may assume that there is a vertex $v$ of $R$ which is $\left(W \backslash\left\{w_{n}\right\}\right)$-complete. Hence $v$ is nonadjacent to $w_{n}$. Since $n \geq 3$ and is odd, and $a-w_{1^{-}} \cdots-w_{n}-v-a$ is not an odd antihole, it follows that $v$ is adjacent to $a$. Consequently $v$ is the unique $\left(W \backslash\left\{w_{n}\right\}\right)$-complete vertex in $R$. From (6) we may assume that $v=b$, and $R$ has length 1 . Choose a step $a_{1}-R_{1}-b_{1}, a_{2}-R_{2}-b_{2}$. Then $b_{1}-a-w_{1}-\cdots-w_{n}-b$ is an odd antipath, of length $\geq 5$. All its internal vertices have neighbours in the connected set $V\left(R^{\prime} \backslash w_{n}\right) \cup\left\{a_{2}\right\}$, and its ends do not. By 2.1 applied in $\bar{G}$, there are adjacent vertices $x, y$ in $V\left(R^{\prime} \backslash w_{n}\right) \cup\left\{a_{2}\right\}$, such that $x-a-w_{1}-\cdots-w_{n}-y$ is an odd antipath. Since $x$ is adjacent to $w_{n}$, it follows that $x$ is the neighbour of $w_{n}$ in $R^{\prime}$, and therefore either $y$ is the second neighbour of $x$ in $R^{\prime}$, or $R^{\prime}$ has length 1 and $y=a_{2}$. Assume first that $R^{\prime}$ has length $>1$, and so both $x, y$ belong to the interior of $R^{\prime}$. Hence $x, y$ are both anticomplete to $A \cup B$, and so $\left((B \cup\{x\}, \emptyset, A \cup\{y\}), a-w_{1}-\cdots-w_{n}\right)$ is a staircase in $\bar{G}$, contradicting that $\left(S, R_{0}\right)$ is strongly maximal. Now assume that $R^{\prime}$ has length 1 . Then $x=r^{\prime}$ and $y=a_{2}$, and $\left(\left(B \cup\left\{r^{\prime}\right\}, \emptyset, A \cup\{b\}\right), a-w_{1^{-}} \cdots-w_{n}\right)$ is a staircase in $\bar{G}$, a contradiction as before. This proves (7).

We may therefore assume that some vertex of $R \backslash b$ is $W$-complete, for otherwise the theorem holds by (7). Let $a-P-p$ be the subpath of $R \backslash b$ such that $p$ is the unique $W$-complete vertex of $P$. Choose $a_{1} \in A$ and $b_{1} \in B$, adjacent (this is possible by (5)). Let us apply 3.2 to the path $p-P-a-a_{1}-b_{1}$, and the even antipath $a-w_{1}-\cdots-w_{n}-a_{1}$. Both ends of the path are complete to the interior of the antipath, so by 3.2 it follows that $P$ has length 2 , and if $q$ denotes its middle vertex then $q$ is nonadjacent to $w_{n}$ and adjacent to $w_{1}, \ldots, w_{n-1}$. But then $\left((B \cup\{p\}, \emptyset, A \cup\{q\}), a-w_{1}-\cdots-w_{n}\right)$ is a staircase in $\bar{G}$, a contradiction. This completes the proof of 13.1 .

13.2 Let $G$ be Berge, containing no appearance of $K_{4}$, no even prism, no 1-breaker and no 2breaker. Let $K=\left(S=(A, C, B), a_{0}-R_{0}-b_{0}\right)$ be a strongly maximal staircase in $G$, and let $x_{1}, \ldots, x_{t}$ be a right-sequence. Then $x_{1}, \ldots, x_{t}$ are all adjacent to $a_{0}$.

Proof. Suppose the theorem is false, and choose $t$ is small as possible such that the statement of the theorem does not hold. So $t \geq 1$, and $x_{1}, \ldots, x_{t-1}$ are all adjacent to $a_{0}$, and $x_{t}$ is not.

(1) $a_{0}-R_{0}-b_{0}$ is not an optimal banister for $b_{0}$.

For suppose it is, and let $a_{0}-w_{1}-\cdots-w_{n}$ be the trajectory of $a_{0}$. Since $R_{0}$ has length $>1$ it follows from 13.1 that $b_{0}$ is the unique $W$-complete vertex of $R_{0}$, where $W=\left\{w_{1}, \ldots, w_{n}\right\}$. Suppose first that $n=1$. Then $b_{0}$ is the unique neighbour of $w_{1}$ in $R_{0}$, and $w_{1}$ has a nonneighbour in $A$, and so by 12.1 it is a right-star. By axiom 3 there is a banister $r-R-w_{1}$ such that the birth of $r$ 
is earlier than $w_{1}$. Since $a_{0}-R_{0}-b_{0}$ is optimal for $b_{0}$, it follows as in the proof of 13.1 that $R$ is disjoint from $R_{0}$, and there are no edges between $R_{0} \backslash a_{0}$ and $R \backslash w_{1}$. Choose an $S$-rung $a_{1}-R_{1}-b_{1}$. Since $a_{1}-a_{0}-R_{0}-b_{0}-w_{1}-R-r-a_{1}$ is not an odd hole it follows that $a_{0}$ has neighbours in $R$. If it has a neighbour different from $r$, then the path from $a_{0}$ to $w_{1}$ with interior in $R \backslash r$ can be completed via $w_{1}-b_{0}-R_{0}-a_{0}$ and via $w_{1}-b_{1}-R_{1}-a_{1}-a_{0}$, and one of the resulting holes is odd, a contradiction. So the unique neighbour of $a_{0}$ in $R$ is $r$. But then we can add $r$ to $A, w_{1}$ to $B$ and the interior of $R$ to $C$, contradicting the maximality of $\left(S, R_{0}\right)$. So $n \geq 2$. Now all of $w_{1}, \ldots, w_{n-1}$ are left-diagonals, and all of $w_{2}, \ldots, w_{n}$ are right-diagonals. But $w_{1}$ is not a right-diagonal, and $w_{n}$ is not a left-diagonal, and $w_{1}$ is not a right-star, contrary to 12.5 . This proves (1).

Now since $a_{0}$ has a nonneighbour in $\left\{x_{1}, \ldots, x_{t}\right\}$, it follows that there is an optimal banister $r-R$ - $b_{0}$ for $b_{0}$. From (1), $r$ has a nonneighbour in $\left\{x_{1}, \ldots, x_{t-1}\right\}$. From the minimality of $t$ (replacing $R_{0}$ by $R$ ) it follows that $R$ has length 1 , and so $r b_{0}$ is an edge. Let $r-w_{1}-\cdots-w_{n}$ be the trajectory of $r$; so $w_{1}$ is earlier than $x_{t}$. Let $W=\left\{w_{1}, \ldots, w_{n}\right\}$; hence $a_{0}$ is $W$-complete. By 13.1, $n$ is odd.

(2) $b_{0}$ is $W$-complete.

For suppose not. Then by 13.1, there exists $i$ with $1 \leq i<n$ such that $r-w_{1}-\cdots-w_{i}-b_{0}$ is an odd antipath. Now $r, w_{1}, \ldots, w_{i-1}$ are all left-diagonals; $w_{1}, \ldots, w_{i}$ are all right-diagonals; $r$ is not a rightdiagonal (since it is a left-star); and $w_{i}$ is not a left-diagonal (since it is nonadjacent to $b_{0}$ ) and not a right- or left-star (since it is $A \cup B$-complete, because $i<n$ ). This contradicts 12.5, and so proves (2).

(3) $a_{0}$ is adjacent to $r$, and $w_{n}$ is a right-star.

Let $a_{1}-R_{1}-b_{1}$ be an $S$-rung with $w_{n}$ nonadjacent to $a_{1}$. Since $a_{0}-r-w_{1}-\cdots-w_{n}-a_{1}-b_{0}-a_{0}$ is not an odd antihole it follows that $a_{0}$ is adjacent to $r$. So each of $r, w_{1}, \ldots, w_{n-1}$ is left-diagonal, each of $w_{1}, \ldots, w_{n}$ is right-diagonal, $r$ is not right-diagonal, $w_{n}$ is not left-diagonal, and the claim follows from 12.5. This proves (3).

(4) There is no $(W \cup\{r\})$-complete vertex in the interior of $R_{0}$.

For suppose there is, $v$ say. Let $a_{1}-R_{1}-b_{1}$ be an $S$-rung. Then $a_{0}-a_{1}-R_{1}-b_{1}-b_{0}$ is an odd path; both its ends are $(W \cup\{r\})$-complete; and the $(W \cup\{r\})$-complete vertex $v$ has no neighbour in its interior, so by 2.2 there is a $(W \cup\{r\})$-complete vertex in $R_{1}$. But $r$ is a left-star and by $(3), w_{n}$ is a right-star, so they have no common neighbour in $R_{1}$, a contradiction. This proves (4).

(5) $n=1$.

For assume $n>1$. Now $R_{0}$ is odd, and both its ends are $(W \cup\{r\})$-complete. Suppose first that $R_{0}$ has length $\geq 5$. By 2.1 and (4) there is a leap; that is, there are two nonadjacent vertices $x, y \in W \cup\{r\}$ joined by an odd path $P$ whose interior is the interior of $R_{0}$. Choose $b_{1} \in B$; then $b_{1}-x-P-y-b_{1}$ is not an odd hole, and so one of $x, y$ is nonadjacent to $b_{1}$. Since $b_{1}$ is $W$-complete, we may assume $y=r$; and hence $x=w_{1}$ since that is the only vertex in $W$ nonadjacent to $r$. Choose $a_{1} \in A$; then since $a_{1}-r-P-w_{1}-a_{1}$ is not an odd hole it follows that $a_{1}$ is not adjacent to $w_{1}$ and so $n=1$. Now assume that $R_{0}$ has length 3 , and let its internal vertices be $x, y$ (in some order). By 2.1 there exists 
an odd antipath $Q$ joining $x, y$ with interior in $W \cup\{r\}$. If $r \notin V(Q)$ then $b_{1}-x-Q-y-b_{1}$ is an odd antihole, where $b_{1} \in B$; and if $w_{n} \notin V(Q)$ then $a_{1}-x-Q-y-a_{1}$ is an odd antihole, where $a_{1} \in A$. Hence we may assume that $x-r-w_{1}-\cdots-w_{n}-y$ is an antipath. We claim that $C=\emptyset$. For suppose there is an $S$-rung $a_{1}-R_{1}-b_{1}$ say of length $>1$. Then $a_{1}-R_{1}-b_{1}-b_{0}-r-a_{1}$ is a hole of length $\geq 6$; and $r-w_{1}-\cdots-w_{n^{-}}-a_{1}$ is an even antipath of length $\geq 4$; and $a_{0}$ is complete to the antipath, and has no other neighbours on the hole; and at least two vertices of the hole are complete to the interior of the antipath, namely $b_{0}$ and $b_{1}$. This contradicts 3.3. So $C=\emptyset$. Hence $\left((B \cup\{x\}, \emptyset, A \cup\{y\}), r-w_{1}-\cdots-w_{n}\right)$ is a staircase in $\bar{G}$, a contradiction. This proves (5).

From (4), (5) we may apply 2.1 to $R_{0}$ and the anticonnected set $\left\{r, w_{1}\right\}$, and since the latter has only two members, 2.1 implies that there is an odd path $P$ joining $r$ and $w_{1}$ with interior equal to the interior of $R_{0}$. From (3), $w_{1}$ is a right-star, and from axiom 3 there is a banister $r^{\prime}-R^{\prime}-w_{1}$ (and we may choose it optimal for $w_{1}$ ) such that the birth of $r^{\prime}$ is earlier than $w_{1}$. Now $R^{\prime}$ is disjoint from $R_{0}$, and there are no edges between $R_{0} \backslash a_{0}$ and $R^{\prime} \backslash w_{1}$; for otherwise there would be a banister from $r^{\prime}$ to $b_{0}$, contradicting that $r-b_{0}$ is optimal for $b_{0}$. Suppose that $r$ has a neighbour in $R^{\prime}$; then the path between $r$ and $w_{1}$ with interior in $R^{\prime}$ can be completed to holes via $w_{1}-b_{0}-r$ and via $w_{1}-P-r$, a contradiction since one of these holes is odd. So $r$ has no neighbour in $R^{\prime}$. Let $r^{\prime}-v_{1}-\cdots-v_{m}$ be the trajectory of $r^{\prime}$. Since $v_{1}, \ldots, v_{m}$ are earlier than $w_{1}$, and $w_{1}$ is the earliest nonneighbour of $r$, it follows that $r$ is adjacent to all of $v_{1}, \ldots, v_{m}$. Now by 13.1, either

- $w_{1}$ is the unique $\left\{v_{1}, \ldots, v_{m}\right\}$-complete vertex in $R^{\prime}$; but then $w_{1}-R^{\prime}-r^{\prime}-a_{1}-r$ (where $a_{1} \in A$ is nonadjacent to $\left.v_{m}\right)$ is an odd path; its ends are $\left\{v_{1}, \ldots, v_{m}\right\}$-complete and its internal vertices are not; and the $\left\{v_{1}, \ldots, v_{m}\right\}$-complete vertex $b_{1}$ (for any $b_{1} \in B$ nonadjacent to $a_{1}$ ) has no neighbour in its interior, contrary to 2.2 .

- $R^{\prime}$ has length 1 , and there is an odd antipath $Q$ between $r^{\prime}$ and $w_{1}$ with interior in $\left\{v_{1}, \ldots, v_{m}\right\}$; but then $r-r^{\prime}-Q-w_{1}-r$ is an odd antihole, a contradiction.

This completes the proof of 13.2 .

Now we are ready to apply 13.2 to produce a skew partition. Let us say a 3-breaker in $G$ is a pair $(K, x)$ such that $K=\left(S=(A, C, B), a_{0}-R_{0}-b_{0}\right)$ is a strongly maximal staircase in $G$, and $x \in V(G) \backslash V(K)$ is $B$-complete, and not $A$-complete, and not $A$-anticomplete.

13.3 Let $G$ be Berge, containing no appearance of $K_{4}$, no even prism, no 1-breaker and no 2-breaker. Suppose that there is a 3-breaker in $G$; then $G$ admits a balanced skew partition.

Proof. Let $\left(K, x_{1}\right)$ be a 3-breaker, where $K=\left(S=(A, C, B), a_{0}-R_{0}-b_{0}\right)$. The 1-vertex sequence $x_{1}$ is a right-sequence; so there exists a right-sequence $x_{1}, \ldots, x_{t}$ of maximum length, with $t \geq 1$. Let $X=\left\{x_{1}, \ldots, x_{t}\right\}$, and let $Y$ be the set of all $A \cup X$-complete vertices in $V(G) \backslash V(S)$. So $a_{0} \in Y$ by 13.2 .

(1) $X \cup Y \cup B$ meets the interior of every path in $G$ from $A \cup C$ to $b_{0}$.

For suppose $P$ is a path from $A \cup C$ to $b_{0}$ with no internal vertex in $X \cup Y \cup B$. Note that $b_{0} \notin X$ by 13.2 , and so $b_{0} \notin X \cup Y \cup B$ (since it is not $A$-complete). We may assume $P$ is minimal, and therefore no internal vertex of $P$ is in $V(S)$. Let $P$ be from $p \in A \cup C$ to $b_{0}$. By $12.3, P \backslash p$ 
contains either a major vertex or a banister. Suppose first that it contains a banister $a-R$ - $b$ say. Hence $a, b \notin X \cup Y \cup B$. Since $a$ is $A$-complete it is therefore not $X$-complete (because it is not in $Y$ ), and then we can set $x_{t+1}=b$, contradicting the maximality of the right-sequence. So $P \backslash p$ contains no banister. Now assume it contains a major vertex $v$ say. Since $v \notin X \cup Y \cup B$, it follows that $v$ is not $X \cup A$-complete. Suppose $v$ is $B$-complete. Since it is major it has a neighbour in $A$. If it is not $A$-complete we can set $x_{t+1}=v$ and obtain a longer right-sequence, a contradiction; and if $v$ is $A$-complete then since it is not $X \cup A$-complete, it is not $X$-complete and so again we can set $x_{t+1}=v$ and obtain a longer right-sequence, a contradiction. So $v$ is not $B$-complete. By 12.1 and since there is no 2-breaker in $G$ and therefore no central vertex, $v$ is left-diagonal, and not right-diagonal; and since it is not $X \cup A$-complete, it is not $X$-complete. Let $v-w_{1}-\cdots-w_{n}$ be the trajectory of $v$. Then each of $w_{1}, \ldots, w_{n}$ is right-diagonal, since they are all $B \cup\left\{a_{0}\right\}$-complete. Since $w_{n}$ has a nonneighbour in $A$, it is not left-diagonal; and so there is a minimum $i$ with $1 \leq i \leq n$ such that $w_{i}$ is not left-diagonal. By 12.5 applied to the sequence $v, w_{1}, \ldots, w_{i}$, we deduce that $v$ is a left-star, contradicting that $v$ is major. This proves (1).

Now since $S$ is step-connected, it follows that $A \cup C$ is connected; and therefore belongs to a component $A_{1}$ of $G \backslash(X \cup Y \cup B)$. Let $A_{2}$ be the union of all the other components. So by (1), $b_{0} \in A_{2}$, and $\left(A_{1} \cup A_{2}, X \cup Y \cup B\right)$ is a skew partition of $G$ (since $Y \cup B$ is complete to $X$, and $X$ is nonempty). We need to find a balanced skew partition. By 4.2 we may assume this skew partition is not loose; so every $X$-complete vertex in $G$ either belongs to $B$ or is also $A$-complete. Every vertex in $Y \cup B$ has a neighbour in $A \cup C$, so $A \cup C$ is a kernel for this skew partition, in $\bar{G}$. By 4.6 it suffices to show that in $G$, any two nonadjacent vertices in $Y \cup B$ are joined by an even path with interior in $A_{1} \cup A_{2}$, and any two adjacent vertices of $A \cup C$ are joined by an even antipath with interior in $X \cup Y \cup B$. Now let $u, v \in Y \cup B$ be nonadjacent. If they are both adjacent to $b_{0}$, then any path joining them with interior in $A \cup C$ (and there is one) is even, since it can be completed to a hole via $v$ - $b_{0}-u$. So we may assume that $u$ is nonadjacent to $b_{0}$, and hence $u \notin B$, so $u \in Y$. If they are both in $Y$, then they are joined by an even path $u-a_{1}-v$ for any $a_{1} \in A$. So we may assume that $v \in B$. Since $u$ is nonadjacent to $b_{0}$ and to $v$, it is neither left- nor right-diagonal, and it is not central since there is no 2-breaker; so from 12.1 it is a left-star. Let $a_{1}-R_{1}-v$ be an $S$-rung; then $u-a_{1}-R_{1}-v$ is the desired even path between $u$ and $v$. Now for antipaths, let $u v$ be an edge with $u, v \in A \cup C$. They both therefore have nonneighbours in $B$, and since $B \cup\left\{a_{0}\right\}$ is anticonnected, they are joined by an antipath $Q$ with interior in $B \cup\left\{a_{0}\right\}$. It suffices to show that $Q$ is even, since $Q^{*} \subseteq Y \cup B$. If $a_{0} \notin Q^{*}$, then $Q$ is even since $b_{0}-u-Q-v-b_{0}$ is an antihole. So $a_{0}$ is in $Q^{*}$. But there are no edges between $a_{0}$ and $B$, and so $a_{0}$ is nonadjacent to every other vertex in the interior of $Q$; and since $Q$ is an antipath, it therefore has at most 3 internal vertices, so its length is $\leq 4$. If it is odd, then it has length 3 , that is, there are nonadjacent vertices $u^{\prime} \in Y$ and $v^{\prime} \in B$, joined by an odd path with interior in $A \cup C$. But we have already shown that they are joined by an even path, and the result follows from 4.3. This proves 13.3.

Now we can prove 1.8.5, the main result of this section. We restate it (proper homogeneous pairs were defined in section 1.)

13.4 Let $G$ be Berge, such that there is no appearance of $K_{4}$ in either $G$ or $\bar{G}$. Suppose that $G$ contains a long odd prism as an induced subgraph. Then either one of $G, \bar{G}$ admits a proper 2-join, or $G$ admits a balanced skew partition, or $G$ admits a proper homogeneous pair. 
Proof. We assume that $G$ does not admit a balanced skew partition, and $G, \bar{G}$ do not admit proper 2-joins. Since $G$ contains a long odd prism, and therefore $G, \bar{G}$ are not even prisms, it follows from 10.6 that $G, \bar{G}$ contain no even prism. By 11.5, 12.4 and 13.3, $G, \bar{G}$ contain no 1-, 2- or 3-breaker.

Since $G$ contains a long odd prism, it contains a staircase; and therefore (possibly by replacing $G$ by its complement) there is a strongly maximal staircase $K=\left(S=(A, C, B), a_{0}-R_{0}-b_{0}\right)$ say in $G$. Let $A_{0}$ be the set of all left-stars, $B_{0}$ the set of all right-stars, and $N$ the set of all vertices that are $A \cup B$-complete. By 12.1, every non-major $A$-complete vertex is in $A_{0}$, and since there is no 3 -breaker, every major $A$-complete vertex is in $N$, so every $A$-complete vertex is in $A_{0} \cup N$; and similarly every $B$-complete vertex is in $B_{0} \cup N$. Let $H=G \backslash\left(V(S) \cup A_{0} \cup B_{0} \cup N\right)$.

(1) Let $F$ be a component of $H$, and let $X$ be the set of attachments of $F$ in $V(S) \cup A_{0} \cup B_{0}$. Then either $X \cap V(S)=\emptyset$, or $X \subseteq V(S)$ and $X$ meets both $A \cup C$ and $B \cup C$.

We may assume that $X$ meets $V(S)$, and therefore from the symmetry we may assume that $X$ meets $A \cup C$. Since no vertex in $F$ is $A$ - or $B$-complete, and therefore no vertex in $F$ is major or a left- or right-star, it follows from 12.3 that $X$ is disjoint from $B_{0}$. If $X$ meets $B \cup C$ then similarly $X$ is disjoint from $A_{0}$, and so $X \subseteq V(S)$ and the claim holds. We assume therefore that $X \subseteq A \cup A_{0}$. Now if $v \in V(G) \backslash F$ has a neighbour in $F$, then $v \notin V(H)$, and so $v \in V(S) \cup A_{0} \cup B_{0} \cup N$, and therefore $v \in X \cup N \subseteq A \cup A_{0} \cup N$. Hence $\left(V(G) \backslash\left(A \cup A_{0} \cup N\right), A \cup A_{0} \cup N\right)$ is a skew partition of $G$, since $F$ is a component of $V(G) \backslash\left(A \cup A_{0} \cup N\right)$ and $b_{0}$ is in a different component, and $A, A_{0} \cup N$ are both nonempty and complete to each other. Now by 2.6, $(B \cup C, A)$ is balanced, since $a_{0}$ is complete to $A$ and anticomplete to $B \cup C$; and therefore from 2.7, $(F, A)$ is balanced (since $B \cup C$ is connected and all vertices in $A$ have neighbours in it). Hence from 4.5, $G$ admits a balanced skew partition, a contradiction. This proves (1).

Let $M$ be the union of all components of $H$ with no attachment in $V(S)$. Then $M$ is nonempty, since by (1) the component of $H$ containing the interior of $R_{0}$ has no attachments in $V(S)$. Let $D$ be the union of all the components of $H$ that have an attachment in $V(S)$. Hence $V(G)$ is partitioned into $A, B, C, D, A_{0}, B_{0}, N, M$, where possibly $C, D$ or $N$ may be empty.

(2) $N \neq \emptyset$.

For assume that $N=\emptyset$. Then the only edges between $V(S) \cup D$ and $A_{0} \cup B_{0} \cup M$ are the edges from $A$ to $A_{0}$ and those from $B$ to $B_{0}$; and since $R_{0}$ is an odd path from $A_{0}$ to $B_{0}$ of length $\geq 3$ and with $V\left(R_{0}\right) \subseteq A_{0} \cup B_{0} \cup M$, and both $A$ and $B$ contain at least two vertices, it follows that $G$ admits a proper 2-join, a contradiction. This proves (2).

(3) $C \cup D=\emptyset$.

For assume that $C \cup D$ is nonempty. By (1) there are no edges between $C \cup D$ and $A_{0} \cup B_{0} \cup M$. Since $N$ is complete to $A \cup B$, it follows that $\left(C \cup D \cup A_{0} \cup B_{0} \cup M, N \cup A \cup B\right)$ is a skew partition of $G$. By 4.2 , it is not loose, and so there is no $N^{\prime}$-complete vertex in $R_{0}$, where $N^{\prime}$ is an anticomponent of $N$. Let $a_{1}-R_{1}-b_{1}, a_{2}-R_{2}-b_{2}$ be a step; then $a_{1}-a_{0}-R_{0}-b_{0}-b_{2}$ is an odd path of length $\geq 5$; its ends are $N^{\prime}$-complete, and its internal vertices are not. By 2.1, there is a leap in $N^{\prime}$, and so there exist nonadjacent $x, y$ in $N$ such that $x-a_{0}-R_{0}-b_{0}-y$ is a path. But then $\left((A \cup\{x\}, C, B \cup\{y\}), a_{0}-R_{0}-b_{0}\right)$ 
is a staircase, contradicting the maximality of $\left(S, R_{0}\right)$. This proves (3).

But then $(A, B)$ is a proper homogeneous pair in $G$. (This is the only place in the entire paper where we use such pairs.) This proves 13.4.

Let us say a graph $G$ is recalcitrant if:

- $G$ is Berge

- $G$ and $\bar{G}$ are not line graphs, and $G$ is not a double split graph

- $G$ and $\bar{G}$ do not admit proper 2-joins, and

- $G$ does not admit a proper homogeneous pair or balanced skew partition.

The remainder of the paper is basically a proof of the following.

13.5 If $G$ is recalcitrant then either $G$ or $\bar{G}$ is bipartite.

Clearly any counterexample to 1.3 is recalcitrant, so 13.5 will imply 1.3 .

On the other hand, for some future applications, it is desirable to keep closer track of which results hold under which hypotheses, instead of just using the blanket "recalcitrant" hypothesis. But at least, for the remainder of the paper we shall only be concerned with Berge graphs $G$ such that in both $G, \bar{G}$ there is no appearance of $K_{4}$ and no long prism; that is, with the members of the class $\mathcal{F}_{5}$ introduced in section 1 . Certainly every recalcitrant graph belongs to $\mathcal{F}_{5}$, by 10.6 and 9.7 .

It turns out that for such graphs, there is a useful strengthening of 2.1 - the second alternative of that theorem can no longer hold.

13.6 Let $G \in \mathcal{F}_{5}$, and let $P$ be a path in $G$ with odd length. Let $X \subseteq V(G) \backslash V(P)$ be anticonnected, such that both ends of $P$ are $X$-complete. Then either:

1. some edge of $P$ is $X$-complete, or

2. $P$ has length 3 and there is an odd antipath joining the internal vertices of $P$ with interior in $X$.

Proof. Let $P$ be $p_{1^{-}} \cdots-p_{n}$. By 2.1 , we may assume that $P$ has length $\geq 5$ and $X$ contains a leap $u, v$ say; so $u-p_{2^{-}} \cdots-p_{n-1}-v$ is a path. But then the three paths $p_{1}-v, u-p_{n}, p_{2^{-}} \cdots-p_{n-1}$ form a long prism, contrary to $G \in \mathcal{F}_{5}$. This proves 13.6 .

There is an analogous strengthening of 2.9 , as follows.

13.7 Let $G \in \mathcal{F}_{5}$, and let $X, Y$ be disjoint nonempty anticonnected subsets of $V(G)$, complete to each other. Let $P$ be a path in $G$ with even length $>0$, with vertices $p_{1}, \ldots, p_{n}$ in order, such that $p_{1}$ is the unique $X$-complete vertex of $P$ and $p_{n}$ is the unique $Y$-complete vertex of $P$. Then $P$ has length 2 and there is an antipath $Q$ between $p_{2}$ and $p_{3}$ with interior in $X$, and an antipath $R$ between $p_{1}$ and $p_{2}$ with interior in $Y$, and exactly one of $Q, R$ has odd length.

Proof. Let us apply 2.9. We may therefore assume that $P$ has length $\geq 4$ and there are nonadjacent $x_{1}, x_{2} \in X$ such that $x_{1}-p_{2}-\cdots-p_{n}-x_{2}$ is a path $P^{\prime}$ say, of odd length $\geq 5$. But the ends of $P^{\prime}$ are $Y \cup\left\{p_{1}\right\}$-complete, and its internal vertices are not, contrary to 13.6. This proves 13.7. 


\section{The double diamond}

We are finished with prisms - we cannot dispose of the prism where all three paths have length 1 (yet), and we have disposed of all others. Now we turn to a different type of subgraph, the double diamond. A double diamond means the graph with eight vertices $a_{1}, \ldots, a_{4}, b_{1}, \ldots, b_{4}$ and with the following adjacencies: every two $a_{i}$ 's are adjacent except $a_{3} a_{4}$, every two $b_{i}$ 's are adjacent except $b_{3} b_{4}$, and $a_{i} b_{i}$ is an edge for $1 \leq i \leq 4$.

Let $G$ be Berge. If $A, B$ are disjoint subsets of $V(G)$, we say a square in $(A, B)$ is a hole $a_{1}-b_{1}-b_{2}-a_{2}-a_{1}$ of length 4 , where $a_{1}, a_{2} \in A$ and $b_{1}, b_{2} \in B$. The pair $(A, B)$ is square-connected if:

- $|A|,|B| \geq 2$,

- for every partition $(X, Y)$ of $A$ with $X, Y$ nonempty, there is a square $a_{1}-b_{1}-b_{2}-a_{2}-a_{1}$ with $a_{1} \in X$ and $a_{2} \in Y$

- for every partition $(X, Y)$ of $B$ with $X, Y$ nonempty, there is a square $a_{1}-b_{1}-b_{2}-a_{2}-a_{1}$ with $b_{1} \in X$ and $b_{2} \in Y$.

It follows that if $(A, B)$ is square-connected then every vertex of $A \cup B$ is in a square. An antisquare is a square in $\bar{G}$; that is, an antihole $a_{1}-b_{1}-b_{2}-a_{2}-a_{1}$ with $a_{1}, a_{2} \in A$ and $b_{1}, b_{2} \in B$; and $(A, B)$ is antisquare-connected if it is square-connected in $\bar{G}$. For strips in which every rung has length 1 (and from now on, those are the only kind of strips we shall need), being square-connected is the same as being step-connected. We have renamed the concepts because we wanted to improve our notation for a step.

We say a quadruple $(A, B, C, D)$ of subsets of $V(G)$ is a cube in $G$ if it satisfies the following conditions:

- $A, B, C, D$ are pairwise disjoint and nonempty

- $A$ is complete to $C$, and $B$ to $D$, and $A$ is anticomplete to $D$, and $B$ to $C$

- $(A, B)$ is square-connected, and $(C, D)$ is antisquare-connected.

If $G$ contains a double diamond, then it contains a cube in which $A, B, C, D$ all have two elements, and that turns out to be the right approach to the double diamond - grow the cube until it is maximal, and analyze how the remainder of $G$ attaches to it. That is our goal in this section. A cube $(A, B, C, D)$ is maximal if there is no cube $\left(A^{\prime}, B^{\prime}, C^{\prime}, D^{\prime}\right)$ with $A \subseteq A^{\prime}, B \subseteq B^{\prime}, C \subseteq C^{\prime}$, and $D \subseteq D^{\prime}$ such that $(A, B, C, D) \neq\left(A^{\prime}, B^{\prime}, C^{\prime}, D^{\prime}\right)$. The subgraph $G \mid(A \cup B \cup C \cup D)$ is called the graph formed by the cube. Note that if $(A, B, C, D)$ is a cube in $G$, then $(C, D, B, A)$ is a cube in $\bar{G}$. (This is very convenient, because it reduces our work by half — we are going to have the usual minor vertices and major vertices, and they switch when we take complements, so whatever we can prove about minor vertices will also give us information about major vertices by going to the complement.)

14.1 Let $G \in \mathcal{F}_{5}$. Let $(A, B, C, D)$ be a maximal cube in $G$, forming $K$, let $v \in V(G) \backslash V(K)$, and let $X$ be the set of neighbours of $v$ in $V(K)$. Then either

- $X$ is a subset of one of $A \cup B, C \cup D, A \cup C, B \cup D$, and $X \cap(A \cup C)$ is complete to $X \cap(B \cup D)$, or 
- $X$ includes one of $A \cup B, C \cup D, A \cup D, B \cup C$, and $(A \cup D) \backslash X$ is anticomplete to $(B \cup C) \backslash X$.

Proof. Note that under taking complements the two outcomes become exchanged. If $X \subseteq A \cup B$, and there exists $a \in X \cap A$ and $b \in X \cap B$, nonadjacent, then choose $c \in C$ and $d \in D$, adjacent, and $v$ - $a-c-d-b-v$ is an odd hole. So if $X \subseteq A \cup B$ then the theorem holds. Similarly it holds if $X \subseteq C \cup D$; and trivially it holds if $X$ is a subset of one of $A \cup C, B \cup D$. So we may assume that $X$ meets both $A$ and $D$. From the same argument in $\bar{G}$, we may also assume that none of $A \cup B, C \cup D, A \cup D, B \cup C$ is a subset of $X$, that is, either $X$ includes neither of $A, C$ or it includes neither of $B, D$. These two cases are exchanged when we pass to the complement; so we may assume by taking complements that $X$ includes neither of $B, D$. Let $A_{1}=A \cap X$, and $A_{2}=A \backslash A_{1}$; and define $B_{1}, B_{2}$ etc. similarly. We have shown so far that $A_{1}, B_{2}, D_{1}, D_{2}$ are nonempty. Choose an antisquare $c_{2}-d_{1}-d_{2}-c_{1}-c_{2}$ such that $d_{1} \in D_{1}$ and $d_{2} \in D_{2}$, and choose $b_{2} \in B_{2}$. Since $v-c_{2}-d_{2}-b_{2}-d_{1}-v$ is not an odd hole, it follows that $c_{2} \in C_{2}$. Hence $A_{1}$ is complete to $B_{1}$; for if $a_{1} \in A_{1}$ and $b_{1} \in B_{1}$ are nonadjacent then $v$ - $a_{1}-c_{2}-d_{2}-b_{1}-v$ is an odd hole. If $A_{1}=A$, then since $(A, B)$ is square-connected and $A_{1}$ is complete to $B_{1}$ it follows that $B_{1}$ is empty; but then we can add $v$ to $C$ (because $v-d_{2}-d_{1}-c_{2}-v$ becomes a new antisquare), contrary to the maximality of the cube. So $A_{2}$ is nonempty. Hence there is a square $a_{1}-b_{1}-b_{2}-a_{2}-a_{1}$ with $a_{1} \in A_{1}$ and $a_{2} \in A_{2}$. Since $a_{1}$ is nonadjacent to $b_{2}$ and complete to $B_{1}$, it follows that $b_{2} \in B_{2}$; but then $v-a_{1}-a_{2}-b_{2}-d_{1}-v$ is an odd hole, a contradiction. This proves 14.1.

Say a vertex $v \in V(G) \backslash V(K)$ is minor if the first case of 14.1 applies to it, and major if the second case applies. Then every such vertex is either minor or major and not both; and by taking complements, the minor and major vertices are exchanged.

14.2 Let $G \in \mathcal{F}_{5}$. Let $(A, B, C, D)$ be a maximal cube in $G$, forming $K$, let $F \subseteq V(G) \backslash V(K)$ be a connected set of minor vertices, and let $X$ be the set of attachments of $F$ in $V(K)$. Then $X$ is a subset of one of $A \cup B, C \cup D, A \cup C, B \cup D$. Moreover, $X \cap(A \cup C)$ is complete to $X \cap(B \cup D)$.

Proof. Suppose the first assertion is false, and choose $F$ minimal with this property. We may assume that $X$ meets both of $A, D$. Since all vertices in $F$ are minor, it follows that $F$ is a path $f_{1}-f_{2^{-}} \cdots-f_{k}$ of length $\geq 1$. We may assume $f_{1}$ is the unique vertex of $F$ with a neighbour in $A$, and $f_{k}$ is the unique vertex of $F$ with a neighbour in $D$. Let $X_{1}, X_{2}$ be the sets of attachments in $V(K)$ of $F \backslash\left\{f_{k}\right\}, F \backslash\left\{f_{1}\right\}$ respectively. From the minimality of $F$ it follows that $X_{1}$ is a subset of one of $A \cup B, A \cup C$, and $X_{2}$ is a subset of one of $B \cup D, C \cup D$.

(1) Not both $X_{1} \subseteq A \cup B$ and $X_{2} \subseteq B \cup D$.

For suppose that both these hold. If $k$ is even, choose $a \in A$ is adjacent to $f_{1}$, and $d \in D$ is adjacent to $f_{k}$, and $c \in C$ is adjacent to $d$; then $a-f_{1^{-}} \cdots-f_{k^{-}} d-c-a$ is an odd hole, a contradiction. So $k$ is odd. Suppose first that $f_{1}$ is complete to $A$. Since it is minor, it has no neighbours in $B$ (for no vertex in $B$ is $A$-complete). If there are no edges between $B$ and $F$, let $a_{1}-b_{1}-b_{2}-a_{2}-a_{1}$ be a square, and let $d \in D$ be adjacent to $f_{k}$; then $a_{1}-b_{1}, a_{2}-b_{2}, f_{1^{-}} \cdots-f_{k^{-}} d$ form a long prism, a contradiction. So there are edges between $B$ and $F$. Choose $i$ with $1 \leq i \leq k$ minimum such that $f_{i}$ has a neighbour in $B$. If $f_{i}$ is not complete to $B$, choose a square $a_{1}-b_{1}-b_{2}-a_{2}-a_{1}$ such that $f_{i}$ is adjacent to $b_{1}$ and not to $b_{2}$; then $b_{1}$ can be linked onto the triangle $\left\{f_{1}, a_{1}, a_{2}\right\}$, via $b_{1}-f_{i^{-}} \cdots-f_{1}, b_{1}-a_{1}, b_{1}-b_{2}-a_{2}$, contrary to 2.4. So $f_{i}$ is complete to $B$. Let $a_{1}-b_{1}-b_{2}-a_{2}-a_{1}$ be a square; then since $a_{1}-b_{1}, a_{2}-b_{2}, f_{1}-\cdots-f_{i}$ do not form a long prism (because $G \in \mathcal{F}_{5}$ ), it follows that $i=2$. But $k>2$ since $k$ is odd; so we can add $f_{1}$ 
to $C$ and $f_{2}$ to $D$, contrary to the maximality of the cube. This proves (1) if $f_{1}$ is $A$-complete. Now assume $f_{1}$ is not $A$-complete, and choose a square $a_{1}-b_{1}-b_{2}-a_{2}-a_{1}$ such that $f_{1}$ is adjacent to $a_{1}$ and not to $a_{2}$. Since $a_{1}-f_{1^{-}} \cdots-f_{k^{-}} d-b_{2}-a_{2}-a_{1}$ is not an odd hole (where $d \in D$ is adjacent to $f_{k}$ ), it follows that $b_{2}$ has a neighbour in $F$. Choose $i$ minimum such that $b_{2}$ is adjacent to $f_{i}$. Let $c \in C$ and $d \in D$ be any adjacent pair of vertices. Then the three paths $a_{1}-b_{1}, a_{2}-b_{2}, c-d$ form a prism, and since the set of attachments of $\left\{f_{1}, \ldots, f_{i}\right\}$ in this prism is not local, and does not include $a_{2}$, it has an attachment in the third path $c$ - $d$, by 10.4; and hence $i=k$, and $f_{k}$ is $D$-complete. Again, let $c \in C$ and $d \in D$ be adjacent. Then the prism formed by $a_{1}-f_{1}-\cdots-f_{k}, a_{2}-b_{2}, c-d$ is long, contrary to $G \in \mathcal{F}_{5}$. This proves (1).

(2) Not both $X_{1} \subseteq A \cup C$ and $X_{2} \subseteq C \cup D$.

For assume these both hold. Choose a square $a_{1}-b_{1}-b_{2}-a_{2}-a_{1}$ such that $f_{1}$ is adjacent to $a_{1}$, and choose $d \in D$ adjacent to $f_{k}$. If $a_{2}$ is adjacent to $f_{1}$ then $a_{1}-b_{1}, a_{2}-b_{2}, f_{1^{-}} \cdots-f_{k^{-}} d$ form a long odd prism, a contradiction. If $a_{2}$ is not adjacent to $f_{1}$ then $a_{1}$ can be linked onto the triangle $\left\{b_{1}, b_{2}, d\right\}$, via $a_{1}-b_{1}, a_{1}-a_{2}-b_{2}, a_{1}-f_{1^{-}} \cdots-f_{k^{-}} d$, a contradiction. This proves (2).

(3) Not both $X_{1} \subseteq A \cup B$ and $X_{2} \subseteq C \cup D$.

For assume these both hold. Then $X_{1} \cap X_{2}=\emptyset$, and so $f_{1}$ is the unique neighbour in $F$ of the vertices in $X_{1}$, and $f_{k}$ is the unique neighbour of those in $X_{2}$. From (1), $X_{2} \nsubseteq \subset \cup D$ and so $X_{2} \cap C \neq \emptyset$; and similarly from (2), $X_{1} \cap B \neq \emptyset$. Also we are given that $X_{1} \cap A, X_{2} \cap D \neq \emptyset$. Since $a_{1}-f_{1}-\cdots-f_{k}-c_{1}-a_{1}$ is a hole (where $a_{1} \in A \cap X_{1}$ and $c_{1} \in C \cap X_{2}$ ) it follows that $k$ is even. Since $f_{1}$ is minor, $X_{1} \cap A$ is complete to $X_{1} \cap B$, and so $A, B$ are not subsets of $X_{1}$; and similarly $C \cap X_{2}$ is complete to $D \cap X_{2}$ and therefore $C, D$ are not subsets of $X_{2}$. So all the eight sets $A \cap X_{1}, A \backslash X_{1}$ etc. are nonempty. Choose a square $a_{1}-b_{1}-b_{2}-a_{2}-a_{1}$ such that $f_{1}$ is adjacent to $a_{1}$ and not to $a_{2}$; and choose an antisquare $c_{1}-d_{1}-d_{2}-c_{2}-c_{1}$ such that $f_{k}$ is adjacent to $d_{1}$ and not to $d_{2}$. It follows that $f_{1}$ is nonadjacent to $b_{2}$, since $X_{1} \cap A$ is complete to $X_{1} \cap B$, and $f_{k}$ is not adjacent to $c_{1}$ since $X_{2} \cap C$ is complete to $X_{2} \cap D$. But then $a_{1}-f_{1^{-}} \cdots-f_{k^{-}} d_{1}-b_{2}-d_{2}-c_{1}-a_{1}$ is an odd hole, a contradiction. This proves (3).

(4) Not both $X_{1} \subseteq A \cup C$ and $X_{2} \subseteq B \cup D$.

For assume both these hold. Then again, the only edges between $V(K)$ and $F$ are between $X_{1}$ and $f_{1}$ and between $X_{2}$ and $f_{k}$. By (1) and (2), again all four of the sets $A \cap X_{1}, B \cap X_{2}, C \cap X_{1}, D \cap X_{2}$ are nonempty. There are two cases, depending on the parity of $k$. First assume $k$ is odd. Then $A \cap X_{1}$ is anticomplete to $B \cap X_{2}$ (for if $a b$ were an edge there, then $a-f_{1^{-}} \cdots-f_{k}-b-a$ would be an odd hole), and so $A \backslash X_{1}, B \backslash X_{2}$ are nonempty; and similarly $C \cap X_{1}$ is anticomplete to $D \cap X_{2}$, and therefore $C \backslash X_{1}, D \backslash X_{2}$ are nonempty. Choose a square $a_{1}-b_{1}-b_{2}-a_{2}-a_{1}$ such that $f_{1}$ is adjacent to $a_{1}$ and not to $a_{2}$, and choose an antisquare $c_{1}-d_{1}-d_{2}-c_{2}-c_{1}$ such that $f_{k}$ is adjacent to $d_{1}$ and not to $d_{2}$. Since $A \cap X_{1}$ is anticomplete to $B \cap X_{2}$ it follows that $b_{1} \notin X_{2}$, and $c_{2} \notin X_{1}$ similarly; and since $a_{1}-f_{1^{-}} \cdots-f_{k^{-}}-d_{1}-b_{2}-a_{2}-a_{1}$ is not an odd hole it follows that $b_{2} \in X_{2}$. But then the three paths $a_{2}-b_{2}, c_{2}-d_{1}, a_{1}-f_{1}-\cdots-f_{k}$ form a long prism, contrary to $G \in \mathcal{F}_{5}$. Now assume $k$ is even. Then $A \cap X_{1}$ is anticomplete to $B \backslash X_{2}$ (for if $a \in A \cap X_{1}$ is adjacent to $b \in B \backslash X_{2}$ then $a-f_{1}-\cdots-f_{k}-d-b$ - $a$ is an odd hole, where $\left.d \in X_{2} \cap D\right)$. Similarly $A \backslash X_{1}$ is anticomplete to $B \cap X_{2}, C \cap X_{1}$ is anticomplete to $D \backslash X_{2}$, and $C \backslash X_{1}$ is anticomplete to $D \cap X_{2}$. Choose $a \in A \cap X_{1}$ and a neighbour $b$ of $a$ in $B$; then $b \in X_{2}$. 
Similarly choose $c \in C \cap X_{1}$ and $d \in D \cap X_{2}$, adjacent. Then the three paths $a-b, c-d, f_{1^{-}} \cdots-f_{k}$ form a prism, and so $k=2$. If $f_{1}$ is $C$-complete then since $C \cap X_{1}=C$ is anticomplete to $D \backslash X_{2}$, it follows that $f_{2}$ is $D$-complete; and then we can add $f_{1}$ to $A$ and $f_{2}$ to $B$, contrary to the maximality of the cube. So $C \nsubseteq X_{1}$. Choose an antisquare $c_{1}-d_{1}-d_{2}-c_{2}-c_{1}$ such that $f_{1}$ is adjacent to $c_{1}$ and not to $c_{2}$. It follows that $f_{2}$ is adjacent to $d_{2}$ and not to $d_{1}$. If $f_{1}$ is $A$-complete, then as before $f_{2}$ is $B$-complete, and we can add $f_{1}$ to $C$ and $f_{2}$ to $D$ (because $f_{1}-d_{1}-f_{2}-c_{2}-f_{1}$ is a new antisquare), a contradiction. So $f_{1}$ has a nonneighbour in $A$, and we can choose a square $a_{1}-b_{1}-b_{2}-a_{2}-a_{1}$ such that $f_{1}$ is adjacent to $a_{1}$ and not to $a_{2}$. It follows that $f_{2}$ is adjacent to $b_{1}$ and not to $b_{2}$. But then $a_{1}-f_{1}-f_{2}-d_{2}-b_{2}-d_{1}-c_{2}-a_{1}$ is an odd hole, a contradiction. This proves (4).

From (1)-(4), the first assertion of the theorem follows. Now let us prove the second assertion. We may assume $X$ meets both $A \cup C$ and $B \cup D$, and so from what we just proved, either $X \subseteq C \cup D$ or $X \subseteq A \cup B$. Suppose first that $X \subseteq C \cup D$. If possible, choose $c \in C \cap X$ and $d \in D \cap X$, nonadjacent, and choose a path $P$ joining them with interior in $F$. Let $a_{1}-b_{1}-b_{2}-a_{1}-a_{1}$ be a square; then the three paths $a_{1}-b_{1}, a_{2}-b_{2}, c-P-d$ form a long prism, a contradiction. So there are no such $c, d$, and the theorem holds.

Now assume that $X \subseteq A \cup B$. Assume $X \cap A$ is not complete to $X \cap B$, and choose a path $a-f_{1^{-}} \cdots-f_{k^{-}} b$, where $a \in A, b \in B$ are nonadjacent and $f_{1}, \ldots, f_{k} \in F$, with $k$ minimum. Since $f_{1}$ is minor, its neighbours in $A$ are complete to its neighbours in $B$, and so $k \geq 2$. Let $A^{\prime}$ be the set of all vertices $a \in A$ such that $a$ is adjacent to $f_{1}$ and there is a nonneighbour $b$ of $a$ in $B$ adjacent to $f_{k}$. By assumption $A^{\prime} \neq \emptyset$. Define $B^{\prime}$ similarly in $B$. If $A^{\prime}=A$ and $B^{\prime}=B$, then $f_{1}$ is $A$-complete, and so there are no edges between $\left\{f_{1}, \ldots, f_{k-1}\right\}$ and $B$, from the minimality of $k$; and similarly $f_{k}$ is $B$-complete and there are no edges between $\left\{f_{2}, \ldots, f_{k}\right\}$ and $A$. Choose a square $a_{1}-b_{1}-b_{2}-a_{2}-a_{1}$; then $a_{1}-b_{1}, a_{2}-b_{2}, f_{1^{-}} \cdots-f_{k}$ form a prism, so $k=2$, and we can add $f_{1}$ to $C$ and $f_{2}$ to $D$, contrary to the maximality of the cube. So we may assume that $A^{\prime} \neq A$. Choose a square $a_{1}-b_{1}-b_{2}-a_{2}-a_{1}$ such that $a_{1} \in A^{\prime}$ and $a_{2} \notin A^{\prime}$. Choose $c \in C$ and $d \in D$, adjacent. Choose $b \in B^{\prime}$ nonadjacent to $a_{1}$ (this exists from the definition of $A^{\prime}$ ). From the minimality of $k, a_{1}-f_{1^{-}} \cdots-f_{k^{-}} b$ is a path. From the hole $a_{1}-f_{1}-\cdots-f_{k}-b-d-c-a_{1}$ we deduce that $k$ is even. Since $b$ is not adjacent to $a_{1}, b$ is different from $b_{1}$. Suppose that $f_{k}$ is adjacent to $b_{2}$. Then the set of attachments of $\left\{f_{1}, \ldots, f_{k}\right\}$ with respect to the prism formed by $a_{1}-b_{1}, a_{2}-b_{2}, c-d$ is not local, and yet it has no attachment in $c-d$, so by 10.4 , both $a_{2}$ and $b_{1}$ are attachments. Since $a_{2}, b_{1}$ are nonadjacent, it follows from the minimality of $k$ and 10.1 that $a_{2}$ is adjacent to $f_{1}$ and $b_{1}$ to $f_{k}$, contradicting that $a_{2} \notin A^{\prime}$.

So $f_{k}$ is not adjacent to $b_{2}$. Then $b$ is different from $b_{2}$. Since $c$ has no neighbour in the connected set $F^{\prime}=\left\{f_{1}, \ldots, f_{k}, b\right\}$, and the set of attachments of $F^{\prime}$ is not local with respect to the prism formed by $a_{1}-b_{1}, a_{2}-b_{2}, c-d$, it follows from 10.4 that $F^{\prime}$ has an attachment in $a_{2}-b_{2}$. If $a_{2}$ is not an attachment then $b_{2}$ is, and from the minimality of $k$ it follows that $b$ is the unique neighbour of $b_{2}$ in $F^{\prime}$; but then $a_{2}-b_{2}, c-d, a_{1}-f_{1^{-}} \cdots-f_{k}-b$ form a long prism, a contradiction. So $a_{2}$ is an attachment of $F^{\prime}$. Since $a_{2}-a_{1}-f_{1}-\cdots-f_{k}-b-a_{2}$ is not an odd hole, $a_{2}$ has a neighbour in $\left\{f_{1}, \ldots, f_{k}\right\}$. If $b_{1}$ also has a neighbour in $\left\{f_{1}, \ldots, f_{k}\right\}$, then (since $a_{2}, b_{1}$ are nonadjacent) from the minimality of $k$ and 10.1 it follows that $a_{2}$ is adjacent to $f_{1}$ and $b_{1}$ to $f_{k}$, and hence $a_{2} \in A^{\prime}$, a contradiction. So $b_{1}$ has no neighbour in $\left\{f_{1}, \ldots, f_{k}\right\}$. Since $a_{1}-f_{1^{-}} \cdots-f_{k}-b-b_{1}-a_{1}$ is not an odd hole it follows that $b_{1}$ is not adjacent to $b$, and therefore has no neighbours in $F^{\prime}$. Let $P$ be the path between $a_{2}$ and $b$ with interior in $F^{\prime}$. From 10.4, $a_{1}$ has a neighbour in $P \backslash a_{2}$. But the only neighbour of $a_{1}$ in $F^{\prime}$ is $f_{1}$, so $f_{1}$ is in $P \backslash a_{2}$, and therefore $f_{1}$ is adjacent to $a_{2}$, and there are no other edges between $a_{2}$ and $F^{\prime}$. Since $a_{2} \notin A^{\prime}$ it follows that $a_{2}$ is adjacent to $b$. But then the set of neighbours of $b$ in the prism 
formed by $a_{1}-b_{1}, a_{2}-b_{2}, c-d$ is not local, and yet none are in the path $a_{1}-b_{1}$, contrary to 10.4 . This proves 14.2 .

The main result of this section is 1.8 .6 , which we restate, the following:

14.3 Let $G \in \mathcal{F}_{5}$. If $G$ contains a double diamond as an induced subgraph, then either one of $G, \bar{G}$ admits a proper 2 -join, or $G$ admits a balanced skew partition. In particular, every recalcitrant graph belongs to $\mathcal{F}_{6}$.

Proof. We may assume that $G, \bar{G}$ do not admit proper 2-joins, and $G$ does not admit a balanced skew partition. Suppose for a contradiction that $G$ contains a double diamond; then it contains a cube, and so there is a maximal cube $(A, B, C, D)$ in $G$, forming $K$. Let $F$ be the set of all minor vertices in $V(G) \backslash V(K)$, and $Y$ the set of all major ones.

(1) Every anticomponent $Y_{1}$ of $Y$ is complete to one of $A \cup B, C \cup D, A \cup D, B \cup C$, and every edge from $A \cup D$ to $B \cup C$ has a $Y_{1}$-complete end.

This is immediate from 14.2 by taking complements.

(2) There is no anticomponent of $Y$ that is complete to $A \cup D$ or $B \cup C$.

For suppose such a component exists, say $Y_{1}$. From the symmetry we may assume it is complete to $A \cup D$. Define $L$ to be the union of $C$ and all components of $F$ with an attachment in $C$, and $M$ to be the union of $B$ and all other components of $F$; and define $X$ to be the set of all $Y_{1}$-complete vertices of $G$ not in $L \cup M$. So all major vertices belong to $Y_{1} \cup X$, and the four sets $L, M, X \cup A \cup D, Y_{1}$ are nonempty and partition $V(G)$; and since $Y_{1}$ is complete to $X \cup A \cup D$, and there are no edges between $L, M$ by 14.2, it follows that $\left(L \cup M, X \cup A \cup D \cup Y_{1}\right)$ is a skew partition of $G$. By 4.2 it is not loose. We claim it is balanced. For by $2.6,(L, D)$ is balanced, since any vertex in $B$ is $D$-complete and $L$-anticomplete. Let $u, v \in L$ be adjacent, and suppose they are joined by an odd antipath $Q_{1}$ with interior in $Y_{1}$. If they both have nonneighbours in $D$, then since $D$ is anticonnected they are also joined by an antipath $Q_{2}$ with interior in $D$, which is also odd since its union with $Q_{1}$ is an antihole, contradicting that $(L, D)$ is balanced. So we may assume that $u$ is $D$-complete. Hence $u \notin C$, and so $u$ belongs to some component $F_{1}$ of $F$ with an attachment in $C$. Since $u$ is minor, all its neighbours in $C$ are adjacent to all its neighbours in $D$, and hence it has no neighbours in $C$; so $v \in F_{1}$. Since $F_{1}$ has an attachment in $C$ and in $D$ (because $u$ has neighbours in $D$ ) it follows that $F$ has no attachments in $A$, and so $u, v$ have no neighbours in $A$. But then $a-u-Q_{1}-v-a$ is an odd antihole (where $a \in A$ ), a contradiction. Next suppose there exist nonadjacent $u, v \in Y_{1}$, joined by an odd path $P$ with interior in $L$. By what we just proved about odd antipaths, it follows that $P$ has length $\geq 5$. Now $A \cup D$ is anticonnected, and there is no $A \cup D$-complete vertex in $L$, since every vertex in $L$ is minor or belongs to $C$. Hence the ends of $P$ are $A \cup D$-complete and its internal vertices are not. But this contradicts 13.6. By 4.5, $G$ admits a balanced skew partition, a contradiction. This proves (2).

(3) There is no component of $F$ such that its set of attachments in $K$ is a subset of one of $A \cup C, B \cup D$.

This follows from (2) by taking complements. 
(4) There do not exist both a component $F_{1}$ of $F$ with set of attachments contained in $A \cup B$ and an anticomponent $Y_{1}$ of $Y$ complete to $A \cup B$; and the same holds with $A \cup B$ replaced by $C \cup D$.

For the first assertion, assume that such $F_{1}, Y_{1}$ exist. Define $M=C \cup D \cup\left(F \backslash F_{1}\right)$, and $X$ to be the set of all $Y_{1}$-complete vertices in $V(G) \backslash\left(M \cup F_{1}\right)$. So $A \cup B \subseteq X$, and the four sets $F_{1}, M, Y_{1}, X$ are all nonempty and form a partition of $V(G)$. Since $Y_{1}$ is complete to $X$ and there are no edges between $F_{1}$ and $M$, it follows that $\left(F_{1} \cup M, Y_{1} \cup X\right)$ is a skew partition of $G$. Choose $a \in A$ and $b \in B$, nonadjacent. By 14.2, not both $a, b$ are attachments of $F_{1}$, and therefore the skew partition is loose, and so by $4.5 \mathrm{G}$ admits a balanced skew partition, a contradiction. This proves the first assertion and the second is proved similarly. This proves (4).

Now if $Y=\emptyset$, then by (3) it follows that $G$ admits a proper 2 -join, a contradiction. So $Y$ is nonempty, and by taking complements, $F$ is nonempty. By (4), passing to the complement if necessary, we may assume that there is no anticomponent of $Y$ that is complete to $A \cup B$. Hence $Y$ is complete to $C \cup D$, by (1) and (2). Since $Y$ is nonempty, it follows from (4) that there is no component $F_{1}$ of $F$ with set of attachments contained in $C \cup D$; so by (3), all attachments of $F$ belong to $A \cup B$. Choose an anticomponent $Y_{1}$ of $Y$. By (3) and 14.2, $Y_{1}$ is not $A$-complete or $B$-complete. Let $X$ be the set of $Y_{1}$-complete vertices in $A \cup B \cup C \cup D$. Let $L$ be the union of $A \backslash X$ and all components of $F$ that have an attachment in $A \backslash X$; and let $M$ be the union of $B \backslash X$ and all other components of $F$. By (1) there are no edges between $A \backslash X$ and $B \backslash X$; and therefore by 14.2, no component of $F$ has attachments in both $A \backslash X$ and $B \backslash X$. Hence there is no edge between $L$ and $M$. Since $L, M, X \cup\left(Y \backslash Y_{1}\right), Y_{1}$ is a partition of $V(G)$, and $Y_{1}$ is complete to $X \cup\left(Y \backslash Y_{1}\right)$, it follows that $\left(L \cup M, X \cup\left(Y \backslash Y_{1}\right) \cup Y_{1}\right)$ is a skew partition of $G$. No vertex of $D$ has a neighbour in $L$, and so it is loose, contrary to 4.2 . Hence there is no such graph $G$. This proves 14.3.

\section{Consequences}

Disposal of the long prism and double diamond has a number of consequences that we develop in this section. First, since we have shown that every minimum imperfect graph is recalcitrant and therefore belongs to $\mathcal{F}_{6}$, the next result (together with 1.5) implies that that no minimum imperfect graph $G$ admits a skew partition. This is essentially Chvátal's skew partition conjecture [6]. (Chvátal actually conjectured that no minimal imperfect graph admits a skew partition, which is slightly stronger.)

\subsection{If $G \in \mathcal{F}_{6}$ admits a skew partition, then $G$ admits a balanced skew partition.}

Proof. Let $(A, B)$ be a skew partition in $G$, which by 4.2 we may assume is not loose. We may assume that there is an odd path $P$ of length $\geq 3$ with ends in $B$ and with interior in $A$. Let $P$ have ends $b_{1}, b_{1}^{\prime}$, and let their neighbours in $P$ be $a_{1}, a_{1}^{\prime}$ respectively. Let $A_{1}$ be the component of $A$ including the interior of $P$, and let $B_{1}$ be the anticomponent of $B$ containing $b_{1}, b_{1}^{\prime}$. Let $A_{2}$ be a second component of $A$, and $B_{2}$ a second anticomponent of $B$. Now the ends of $P$ are $B_{2}$-complete, and its internal vertices are not, since the skew partition is not loose; suppose that $P$ has length at least 5 . Then by $2.1, B_{2}$ contains a leap $x, y$ for $P$, and then the subgraph induced on $V(P) \cup\{x, y\}$ is a long prism, a contradiction since $G \in \mathcal{F}_{6}$. So no such path has length $\geq 5$; and similarly no odd antipath with ends in $A$ and interior in $B$ has length $\geq 5$. Hence $P$ has vertices $b_{1}-a_{1}-a_{1}^{\prime}-b_{1}^{\prime}$ in order. 
Now $a_{1}, a_{1}^{\prime}$ both have non-neighbours in $B_{2}$, and hence are joined by an antipath with interior in $B_{2}$; this antipath is odd, since its union with $b_{1}, b_{1}^{\prime}$ induces an antihole, and since all such antipaths have length 3 it follows that there exist nonadjacent $b_{2}, b_{2}^{\prime} \in B_{2}$ such that $b_{2}-a_{1}-a_{1}^{\prime}-b_{2}^{\prime}$ is a path. Now $b_{1}, b_{1}^{\prime}$ both have neighbours in $A_{2}$, since the skew partition is not loose, and hence are joined by a path with interior in $A_{2}$, and it is odd as usual, and hence has length 3 ; so there exist adjacent $a_{2}, a_{2}^{\prime} \in A_{2}$ such that $b_{1}-a_{2}-a_{2}^{\prime}-b_{1}^{\prime}$ is a path. Since $b_{2}-b_{1}-a_{2}-a_{2}^{\prime}-b_{1}^{\prime}-b_{2}$ is not an odd hole, $b_{2}$ is adjacent to one of $a_{2}, a_{2}^{\prime}$, and similarly so is $b_{2}^{\prime}$. But $b_{2}, b_{2}^{\prime}$ have no common neighbour in $A_{2}$, for if $v \in A_{2}$ were adjacent to them both then $v-b_{2}-a_{1}-a_{1}^{\prime}-b_{2}^{\prime}-v$ would be an odd hole. So there are exactly two edges between $\left\{a_{2}, a_{2}^{\prime}\right\}$ and $\left\{b_{2}, b_{2}^{\prime}\right\}$, forming a 2-edge matching. There are two possible pairings; in one case the subgraph induced on these eight vertices is a double diamond, and in the other it is $L\left(K_{3,3} \backslash e\right)$. In both cases this contradicts that $G \in \mathcal{F}_{6}$. This proves 15.1 .

Consequently we have the following:

15.2 Let $G \in \mathcal{F}_{6}$, and assume that $G$ admits no balanced skew partition. Let $X, Y \subseteq V(G)$ be nonempty, disjoint, and complete to each other.

- If $X \cup Y=V(G)$, then either $G$ is complete, or $\bar{G}$ has exactly two components, both with $\leq 2$ vertices (and hence $|V(G)| \leq 4$ ).

- If $X \cup Y \neq V(G)$, then $V(G) \backslash(X \cup Y)$ is connected, and if in addition $|X|>1$, then every vertex in $X$ has a neighbour in $V(G) \backslash(X \cup Y)$.

Proof. By 15.1, $G$ admits no skew partition. Assume first that $X \cup Y=V(G)$. Then $\bar{G}$ is not connected; let the anticomponents of $G$ be $B_{1}, \ldots, B_{k}$ say, where $k \geq 2$. We may assume that $G$ is not complete, and therefore we may assume that some $B_{i}$, say $B_{1}$, has cardinality $>1$. Choose $x, y \in B_{1}$, nonadjacent. Then $(\{x, y\}, V(G) \backslash\{x, y\})$ is not a skew partition, and so $G \backslash\{x, y\}$ is anticonnected. Hence $k=2$ and $B_{1}=\{x, y\}$. Similarly $B_{2}$ has cardinality $\leq 2$, and so $|V(G)| \leq 4$ and the theorem holds. Now assume that $G \backslash(X \cup Y)$ is nonnull. Suppose that $V(G) \backslash(X \cup Y)$ is not connected; then $(V(G) \backslash(X \cup Y), X \cup Y)$ is a skew partition, a contradiction. So $V(G) \backslash(X \cup Y)$ is connected. Now suppose some $x \in X$ has no neighbour in $V(G) \backslash(X \cup Y)$. Hence $V(G) \backslash((X \backslash\{x\}) \cup Y)$ is not connected, and since $G$ admits no skew partition it follows that $X=\{x\}$. This proves 15.2.

Here is another consequence:

15.3 Let $G \in \mathcal{F}_{6}$. Let $C$ be a cycle in $G$ of length $\geq 6$, with vertices $p_{1}, \ldots, p_{n}$ in order, and let $1<h<i$ and $i+1<j<n$. Let $C$ be induced except possibly for an edge $p_{h} p_{j}$. Let $Y \subseteq V(G) \backslash V(C)$ be anticonnected, such that the only $Y$-complete vertices in $C$ are $p_{n}, p_{1}, p_{i}, p_{i+1}$. Suppose there is a path $F$ of $G \backslash Y$ from $p_{h}$ to $p_{j}$ (possibly of length 1 ), such that there are no edges between its interior and $V(C) \backslash\left\{p_{h}, p_{j}\right\}$. Then some vertex of $F$ is $Y$-complete.

Proof. Assume no vertex of $F$ is $Y$-complete. Since the hole

$$
p_{1}-\cdots-p_{h^{-}}-F-p_{j^{-}} \cdots-p_{n}-p_{1}
$$

is even, and the path $p_{1}-\cdots-p_{h^{-}} \cdots-p_{i}$ is even (by 2.2), it follows that the path

$$
p_{i}-p_{i-1^{-}} \cdots-p_{h^{-}}-F-p_{j^{-}} \cdots-p_{n}
$$


is odd, and therefore has length 3 by 13.6. So $F$ has length 1 , and $i=h+1$ and $n=j+1$. Similarly $h=2$ and $j=i+2$, and so $n=6$. Then $p_{2}, p_{5}$ are adjacent, so there is an antipath $Q$ joining them with interior in $Y$. But then in $\bar{G}$, the three paths $p_{1-} p_{4}, p_{5}-p_{2}, p_{3}-Q-p_{6}$ form a long prism, a contradiction. This proves 15.3 .

There is a variant of 3.2 , the following.

15.4 Let $G \in \mathcal{F}_{6}$, and let $p_{1}-\cdots-p_{m}$ be a path in $G$. Let $2 \leq s \leq m-2$, and let $p_{s}-q_{1}-\cdots-q_{n}-p_{s+1}$ be an antipath, where $n \geq 2$. Assume that $p_{1}, p_{m}$ are both adjacent to all of $q_{1}, \ldots, q_{n}$. Then $n$ is even and $m=4$.

Proof. If $n$ is even then $p_{s}-q_{1}-\cdots-q_{n}-p_{s+1}$ is an odd antipath, and $p_{1}, p_{m}$ are complete to its interior; and hence $p_{1}, p_{m}$ are both adjacent to one of $p_{s}, p_{s+1}$. So $s=2$ and $m=s+2$, and therefore $m=4$. Now assume $n$ is odd; then $p_{s}-q_{1}-\cdots-q_{n}-p_{s+1}$ is an even antipath of length $\geq 4$, contrary to 13.7 applied in $\bar{G}$ to this antipath and the sets $\left\{p_{1}, \ldots, p_{s-1}\right\},\left\{p_{s+2}, \ldots, p_{n}\right\}$. This proves 15.4.

There is a strengthening of 2.3 :

15.5 Let $G \in \mathcal{F}_{6}$, let $C$ be a hole in $G$, and let $X \subseteq V(G) \backslash V(C)$ be anticonnected. Let $P$ be a path in $C$ of length $>1$ such that its ends are $X$-complete and its internal vertices are not. Then $P$ has even length.

Proof. The claim is trivial if $C$ has length 4 , so we assume it has length $\geq 6$. Let the vertices of $C$ be $p_{1}, \ldots, p_{n}$ in order, and let $P$ be $p_{1}-\cdots-p_{k}$ say, where $3 \leq k<n$. Assume $k$ is even. Then by 13.6 applied to $P$ we deduce that $P$ has length 3 , so $k=4$. By 2.2 every $X$-complete vertex is adjacent to one of $p_{2}, p_{3}$, so there are none in the interior of the odd path $p_{4}-p_{5^{-}} \cdots-p_{n}-p_{1}$. By 13.6 this path also has length 3 , so $n=6$. Let $Q$ be the shortest antipath with interior in $X$, joining either $p_{2}, p_{3}$ or $p_{5}, p_{6}$. From the symmetry we may assume its vertices are $p_{2}-q_{1}-\cdots-q_{m}-p_{3}$ say. Then $Q$ is odd since it can be completed to an antihole via $p_{3}-p_{1}-p_{4}-p_{2}$; and since $p_{5}-p_{2}-Q-p_{3}-p_{5}$ is therefore not an antihole, it follows that $p_{5}$ (and similarly $p_{6}$ ) has a nonneighbour in the interior of $Q$. From the choice of $Q$ it follows that $p_{5}, p_{6}$ both have exactly one nonneighbour in the interior of $Q$; one is nonadjacent to $q_{1}$ and the other to $q_{m}$. Suppose that $m>2$. If $p_{5}$ is nonadjacent to $q_{1}$ then the three antipaths $q_{1}-\cdots-q_{m}, p_{5}-p_{3}, p_{2}-p_{6}$ for a long prism in $\bar{G}$, contrary to $G \in \mathcal{F}_{6}$; while if $p_{5}$ is nonadjacent to $q_{m}$ then $q_{1}-\cdots-q_{m}, p_{6}-p_{3}, p_{2}-p_{5}$ form a long prism, again a contradiction. So $m=2$. But then $G \mid\left\{p_{1}-\cdots-p_{6}, q_{1}, q_{2}\right\}$ is $L\left(K_{3,3} \backslash e\right)$ if $p_{5}$ is nonadjacent to $q_{1}$, and a double diamond if $p_{5}$ is nonadjacent to $q_{2}$, again contrary to $G \in \mathcal{F}_{6}$. This proves 15.5 .

There is also a strengthening of 3.3; we no longer need the vertex $z$.

15.6 Let $G \in \mathcal{F}_{6}$, let $C$ be a hole in $G$ of length $\geq 6$, with vertices $p_{1}, \ldots, p_{m}$ in order, and let $Q$ be an antipath with vertices $p_{1}, q_{1}, \ldots, q_{n}, p_{2}$, with length $\geq 4$ and even. There is at most one vertex in $\left\{p_{3}, \ldots, p_{m}\right\}$ complete to either $\left\{q_{1}, \ldots, q_{n-1}\right\}$ or $\left\{q_{2}, \ldots, q_{n}\right\}$, and any such vertex is one of $p_{3}, p_{m}$.

Proof. Suppose first that one of $q_{1}, \ldots, q_{n}$ belongs to the hole. Since it is adjacent to at least one of $p_{1}, p_{2}$ (since $Q$ is an antipath), we may assume that it is $p_{m}$; and since it is nonadjacent to $p_{2}$, it follows that $p_{m}=q_{n}$. So $p_{3} \neq q_{1}$ ( since $q_{1}$ is adjacent to $q_{n}$ ), and therefore no more of $q_{1}, \ldots, q_{n}$ belong to $C$. Suppose that there exists $i$ with $3 \leq i<m$ such that $p_{i}$ is complete to either $\left\{q_{1}, \ldots, q_{n-1}\right\}$ or $\left\{q_{2}, \ldots, q_{n}\right\}$. If $i<m-1$ then $p_{i}$ is not adjacent to $p_{m}=q_{n}$, so $p_{i}$ is complete 
to $\left\{q_{1}, \ldots, q_{n-1}\right\}$; but then $p_{i}-p_{1}-q_{1}-\cdots-q_{n}-p_{i}$ is an odd antihole. So $i=m-1$. By 15.5 applied to the path $p_{m-1}-p_{m}-p_{1}-p_{2}$ it follows that $p_{m-1}$ is not complete to $\left\{q_{1}, \ldots, q_{n-1}\right\}$, and therefore it is complete to $\left\{q_{2}, \ldots, q_{n}\right\}$ and nonadjacent to $q_{1}$. But then $p_{2}-p_{m-1}-q_{1}-\cdots-q_{n}-p_{2}$ is an odd antihole, a contradiction. So there is no such $i$, and therefore the theorem holds in this case.

So we may assume that none of $q_{1}, \ldots, q_{n}$ belong to $C$. Let $X=\left\{q_{1}, \ldots, q_{n}\right\}$, and let $Y_{1}, Y_{2}$ be the sets of vertices in $\left\{p_{3}, \ldots, p_{m}\right\}$ complete to $X \backslash\left\{q_{n}\right\}, X \backslash\left\{q_{1}\right\}$ respectively.

(1) $Y_{1} \subseteq Y_{2} \cup\left\{p_{m}\right\}$, and $Y_{2} \subseteq Y_{1} \cup\left\{p_{3}\right\}$.

This is proved as in the proof of 3.3 .

(2) If $Y_{1} \nsubseteq\left\{\left\{p_{m}\right\}\right.$ then $p_{3} \in Y_{1} \cap Y_{2}$, and if $Y_{2} \nsubseteq\left\{p_{3}\right\}$ then $p_{m} \in Y_{1} \cap Y_{2}$.

For assume $Y_{1} \nsubseteq\left\{p_{m}\right\}$, and choose $i$ with $3 \leq i \leq m-1$ minimum so that $p_{i} \in Y_{1}$. By (1), $p_{i} \in Y_{2}$, so we may assume $i>3$, for otherwise the claim holds. By 15.5 applied to the anticonnected set $X \backslash\left\{q_{n}\right\}, i$ is even. The path $p_{1}-\cdots-p_{i}$ is odd, and between $X \backslash\left\{q_{1}\right\}$-complete vertices, so by 15.5 it contains another in its interior, say $p_{h}$. From the minimality of $i, p_{h} \notin Y_{1}$, so by (1) $h=3$, and 15.5 applied to the path $p_{3^{-}} \cdots-p_{i}$ implies that $i=4$. Choose $j$ with $4 \leq j \leq m$ maximum such that $p_{j} \in Y_{2}$. By (1), $p_{j}$ is $X$-complete. By 15.4 applied to $p_{j^{-}} \cdots-p_{m^{-}}-p_{1^{-}} \cdots-p_{4}$ we deduce that $j \leq 5$, and so $j \neq m$. By 15.5 applied to the path $p_{j}-\cdots-p_{m}-p_{1}$ and anticonnected set $X \backslash\left\{q_{1}\right\}$, it follows that $j$ is odd, and so $j=5$. From 15.5 applied to the path $p_{5}-\cdots-p_{m}-p_{1}-p_{2}$ and anticonnected set $X \backslash\left\{q_{n}\right\}$, we deduce that there exists $k$ with $6 \leq k \leq m$ such that $p_{k} \in Y_{1}$. Since it is not in $Y_{2}$, it follows from (1) that $k=m$, and so $p_{m} \in Y_{1} \backslash Y_{2}$. But then $p_{3}-q_{1}-\cdots-q_{n}-p_{m}-p_{3}$ is an odd antihole, a contradiction. This proves (2).

Now not both $p_{3}, p_{m}$ are in $Y_{1} \cap Y_{2}$, for otherwise $Q$ could be completed to an odd antihole via $p_{2}-p_{m}-p_{3}-p_{1}$. Hence we may assume $p_{3} \notin Y_{1} \cap Y_{2}$, and so from (2), $Y_{1} \subseteq\left\{p_{m}\right\}$. By (1), $Y_{2} \subseteq\left\{p_{3}\right\} \cup Y_{1}$, and so $Y_{1} \cup Y_{2} \subseteq\left\{p_{3}, p_{m}\right\}$. We may therefore assume that $Y_{1} \cup Y_{2}=\left\{p_{3}, p_{m}\right\}$, for otherwise the theorem holds. In particular, $p_{3} \in Y_{2}$. If also $p_{m} \in Y_{2}$, then $p_{3}-p_{4^{-}} \cdots-p_{m}$ is an odd path between $X \backslash\left\{q_{1}\right\}$-complete vertices, and none of its internal vertices are $X \backslash\left\{q_{1}\right\}$-complete, contrary to 15.5. So $p_{m} \notin Y_{2}$, and so $p_{m} \in Y_{1}$; but then $p_{3}-q_{1}-q_{2}-\cdots-q_{n}-p_{m}-p_{3}$ is an odd antihole, a contradiction. This proves 15.6 .

This implies a strengthening of 3.1:

15.7 Let $G \in \mathcal{F}_{6}$. Let $C$ be a hole of length $>4$ and $D$ an antihole of length $>4$. Then $\mid V(C) \cap$ $V(D) \mid \leq 2$.

Proof. Assume that $|V(C) \cap V(D)| \geq 3$; then by taking complements if necessary, we may assume that there are three vertices in $V(C) \cap V(D)$ such that exactly one pair of them is adjacent. Hence we can number the vertices of $C$ as $p_{1}, \ldots, p_{m}$ in order, and the vertices of $D$ as $p_{1}, q_{1}, \ldots, q_{n}, p_{2}, p_{k}$ for some $k$ with $4 \leq k \leq m-1$. (Possibly the hole and antihole also share some fourth vertex.) Hence the antipath $p_{1}-q_{1}-\cdots-q_{n}-p_{2}$ has length $\geq 4$ and even. The vertex $p_{k}$ is complete to $\left\{q_{1}, \ldots, q_{n}\right\}$, and different from $p_{3}, p_{m}$, contrary to 15.6. This proves 15.7 . 


\section{Odd wheels}

Now we begin the third of the major parts of the proof, handling Berge graphs that do not contain appearances of $K_{4}$, long prisms or double diamonds, but do contain wheels. A wheel in a graph $G$ is a pair $(C, Y)$, satisfying:

- $C$ is a hole of length $\geq 6$

- $Y$ is a non-null anticonnected set disjoint from $C$

- there are two disjoint $Y$-complete edges of $C$.

We need to study how the remainder of a recalcitrant graph can attach onto a wheel. (Conforti, Cornuéjols, Vušković and Zambelli [11] also made such a study, and their paper contains results related to ours.) We call $C$ the rim and $Y$ the $h u b$ of the wheel. A maximal path in a path or hole $H$ whose vertices are all $Y$-complete is called a segment or $Y$-segment of $H$. A wheel $(C, Y)$ is odd if some segment has odd length. Odd wheels are much easier to handle than general wheels, and in this section we prove that there are no odd wheels in a recalcitrant graph. (Gérard Cornuéjols informs us that he and his co-workers proved the same result, independently, but, like us, assuming the truth of 13.4 - see [11].)

Let us say that distinct vertices $u, v$ of the rim of a wheel $(C, Y)$ have the same wheel-parity if there is a path of the rim joining them containing an even number of $Y$-complete edges (and hence by 2.3 , so does the second path, if $u, v$ are nonadjacent); and opposite wheel-parity otherwise. In any odd wheel $(C, Y)$, there are vertices $u, v$ in $C$ of opposite wheel-parity that are not $Y$-complete, and we shall show that, if the odd wheel has been chosen optimally, then $Y$ and its common neighbours separate $u, v$ and thereby give us a balanced skew partition.

16.1 Let $G \in \mathcal{F}_{6}$, and let $(C, Y)$ be a wheel in $G$. Let $v \in V(G) \backslash(V(C) \cup Y)$, such that $v$ is not $Y$-complete. Suppose that there exist neighbours of $v$ in $C$ with opposite wheel-parity. Then in every path of $C$ between them there is a $Y \cup\{v\}$-complete edge. Moreover, either:

- $v$ has only two neighbours in $C$, and they are adjacent and both $Y$-complete, or

- there is a 3-vertex path $p_{1}-p_{2}-p_{3}$ in $C$, such that $p_{1}, p_{2}, p_{3}$ are all $Y \cup\{v\}$-complete, and every other neighbour of $v$ in $C$ has the same wheel-parity as $p_{1}$, or

- $(C, Y \cup\{v\})$ is a wheel.

\section{Proof.}

(1) Let $P$ be a path in $C$ of length $\geq 1$, such that its ends are adjacent to $v$ and have opposite wheel-parity. Then either some internal vertex of $P$ is a neighbour of $v$, or $P$ has length 1.

For let $C$ have vertices $p_{1}, \ldots, p_{n}$ in order, and let $P$ be the path $p_{1}-\cdots-p_{j}$ say, where $j<n$. We assume no internal vertex of $P$ is a neighbour of $v$, and that $j \geq 3$. From the hole $v-p_{1}-\cdots-p_{j}-v$ it follows that $j$ is odd. Since $p_{1}, p_{j}$ have opposite wheel-parity with respect to $(C, Y)$, there are an odd number of $Y$-complete edges in $P$. Choose $Y^{\prime} \subseteq Y$ minimal such that $Y^{\prime}$ is anticonnected and there are an odd number of $Y^{\prime}$-complete edges in $P$. From 2.3 applied to the hole $v-p_{1^{-}} \cdots-p_{j}-v$, 
it contains just one $Y^{\prime}$-complete edge and only two $Y^{\prime}$-complete vertices. Hence there exists $i$ with $1 \leq i<j$ such that $p_{i}, p_{i+1}$ are the only $Y^{\prime}$-complete vertices in $P$. Since $j$ is odd, it follows that exactly one of $i-1, j-i$ is even; so (by replacing $P$ by its reverse if necessary) we may assume that $i$ is odd. So $p_{j}$ is different from $p_{i+1}$, and hence $p_{j}$ is not $Y^{\prime}$-complete. There are two disjoint $Y^{\prime}$-complete edges in $C$, so one of them does not use $p_{i}$; and therefore it does not use $p_{1}$ either (for $p_{1}$ is not $Y^{\prime}$-complete unless $\left.i=1\right)$. Hence both its ends are in $\left\{p_{j+1}, \ldots, p_{n}\right\}$. Consequently $n \geq j+2$, and since $n$ is even and $j$ is odd it follows that $n \geq j+3$. Therefore there is a $Y^{\prime}$-complete vertex in $\left\{p_{j+2}, \ldots, p_{n-1}\right\}$.

Suppose that $v$ has a neighbour in $\left\{p_{j+2}, \ldots, p_{n-1}\right\}$. Then there is a path $Q$ from $v$ to a $Y^{\prime}$ complete vertex $u$ say, with $V(Q) \subseteq\left\{v, p_{j+2}, \ldots, p_{n-1}\right\}$, such that no internal vertex of $Q$ is $Y^{\prime}$ complete. The path $p_{i^{-}} \cdots-p_{1}-v-Q-u$ has both ends $Y^{\prime}$-complete, and no internal vertex $Y^{\prime}$-complete, and the $Y^{\prime}$-complete vertex $p_{i+1}$ has no neighbour in its interior; so this path is even, that is, $Q$ is odd. Hence the path $p_{i+1^{-}} \cdots-p_{j}-v-Q-u$ is odd, and so by 13.6 has length 3 ; and hence $j=i+2$ and $Q$ has length 1 . Also, every $Y^{\prime}$-complete vertex is adjacent to one of $p_{j}, v$, by 2.2 ; and so $p_{i}$ is adjacent to $v$, and so $i=1, j=3$; and $v$ is adjacent to every $Y^{\prime}$-complete vertex in $C$ except $p_{2}$ and possibly $p_{4}$ (for no others are adjacent to $p_{3}$ ). In particular, there are two nonadjacent $Y^{\prime} \cup\{v\}$-complete vertices in $C$, and so by 2.3 there are an even number of $Y^{\prime} \cup\{v\}$-complete edges in $C$. But all $Y^{\prime}$-complete edges of $C$ are $Y^{\prime} \cup\{v\}$-complete except $p_{1} p_{2}$ and possibly $p_{4} p_{5}$; and since there are also an even number of $Y^{\prime}$-complete edges in $C$, it follows that $p_{4}, p_{5}$ are $Y^{\prime}$-complete, and $v$ is adjacent to $p_{5}$ and not to $p_{4}$. But then the vertices $v, p_{1}, p_{2}, p_{3}, p_{4}, p_{5}$ violate 15.3.

This proves that $v$ has no neighbour in $\left\{p_{j+2}, \ldots, p_{n-1}\right\}$. Choose $k$ with $j \leq k \leq n$ minimum such that $p_{k}$ is $Y^{\prime}$-complete. Since there is a $Y^{\prime}$-complete vertex in $\left\{p_{j+2}, \ldots, p_{n-1}\right\}$, it follows that $k<n$. From 2.3 it follows that the path $p_{i+1^{-}} \cdots-p_{k}$ is even, and so $k$ is even. Suppose that $v$ is not adjacent to $p_{j+1}$. Since $v-p_{j^{-}} \cdots-p_{n}-v$ is not an odd hole, it follows that $v$ is not adjacent to $p_{n}$, so $p_{1}, p_{j}$ are its only neighbours in $C$. But $p_{i^{-}} \cdots-p_{1^{-}}-p_{j^{-}} \cdots-p_{k}$ is odd, and therefore has length 3 by 13.6 ; and by 2.2 , every $Y^{\prime}$-complete vertex in $C$ is adjacent to $v$ except possibly $p_{j-1}, p_{j+1}$, a contradiction since there is a $Y^{\prime}$-complete vertex in $\left\{p_{j+2}, \ldots, p_{n-1}\right\}$. So $v$ is adjacent to $p_{j+1}$. Since $v-p_{j+1^{-}} \cdots-p_{n}-p_{1}-v$ is not an odd hole, it follows that $v$ is also adjacent to $p_{n}$, so it has exactly four neighbours in $C$. Choose $m$ with $k \leq m \leq n$ maximum such that $p_{m}$ is $Y^{\prime}$-complete. It follows that $m \geq j+2$. If $m=n$ then a $Y^{\prime}$-complete vertex in $\left\{p_{j+2}, \ldots, p_{n-1}\right\}$ has no neighbours in the interior of the odd path $p_{i+1^{-}} \cdots-p_{j}-v-p_{n}$, and the ends of this path are $Y^{\prime}$-complete and its internal vertices are not, contrary to 2.2. So $m<n$. Then 2.3 applied to the path $p_{m^{-}} \cdots-p_{n}-p_{1^{-}} \cdots-p_{i}$ implies that $m$ is odd, and therefore $m>k$. Suppose that $m>k+1$. Then $p_{m^{-}} \cdots-p_{n}-v-p_{j+1^{-}} \cdots-p_{k}$ is an odd path, and $p_{i+1}$ has no neighbour in its interior, contrary to 2.2 . So $m=k+1$, and there is symmetry between the paths $p_{1}-\cdots-p_{j}$ and $p_{j+1^{-}} \cdots-p_{n}$. Both these paths have length $\geq 2$; suppose they both have length 2. Then $n=6$, and the only $Y^{\prime} \cup\{v\}$-complete vertices in $C$ are $p_{1}, p_{4}$, contrary to 15.5. So one of the paths has length $>2$, and from the symmetry we may assume that $j \geq 4$. Hence the hole $H=v-p_{1}-\cdots-p_{j}-v$ has length $\geq 6$, and the only $Y^{\prime}$-complete vertices in it are $p_{i}, p_{i+1}$. By 2.10, $Y^{\prime}$ contains a hat or a leap. But $p_{k+1}$ has no neighbour in this hole, so the pair $\left(V(H), Y^{\prime}\right)$ is balanced by 2.6 , and hence there is no leap. So there is a hat; that is, there exists $y \in Y^{\prime}$ with no neighbours in $H$ except $p_{i}, p_{i+1}$. From the minimality of $Y^{\prime}$ it follows that $Y^{\prime}=\{y\}$. But then $G \mid(V(C) \cup\{v, y\})$ is the line graph of a bipartite subdivision of $K_{4}$, a contradiction. This proves (1).

From (1) the first assertion of the theorem follows. Now we prove the second assertion. Suppose that $v$ has at least four neighbours in $C$, two with the same wheel-parity, and two others with the 
opposite wheel-parity. Then there are two disjoint paths as in (1), and therefore from (1) there are two disjoint $Y \cup\{v\}$-complete edges in $C$, and so $(C, Y \cup\{v\})$ is a wheel and the theorem holds. So we may assume that $C$ has vertices $p_{1}, \ldots, p_{n}$ in order, and $v$ is adjacent to $p_{1}$, and $v$ has no other neighbour in $C$ with the same wheel-parity as $p_{1}$. Since $v$ has at least one other neighbour, we may assume it has a neighbour in $V(C) \backslash\left\{p_{1}, p_{n}\right\}$. Choose $i>1$ minimum such that $v$ is adjacent to $p_{i}$; then $i<n$, so by (1), $i=2$. So $p_{2}$ is $Y \cup\{v\}$-complete. If $v$ has a third neighbour in $C$ then similarly $p_{n}$ is $Y \cup\{v\}$-complete and the theorem holds; and if not then again the theorem holds. This proves 16.1 .

16.2 Let $G \in \mathcal{F}_{6}$, and let $(C, Y)$ be a wheel in $G$. Let $F \subseteq V(G) \backslash(V(C) \cup Y)$ be connected, such that no vertex in $F$ is $Y$-complete. Let $X \subseteq V(C)$ be the set of attachments of $F$ in $C$. Suppose that there exist vertices in $X$ with opposite wheel-parity, and there are two vertices in $X$ that are nonadjacent. Then either:

- there is a vertex $v \in F$ such that $(C, Y \cup\{v\})$ is a wheel, or

- there is a vertex $v \in F$ with at least four neighbours in $C$, and a 3-vertex path $p_{1}-p_{2}-p_{3}$ in $C$, such that $p_{1}, p_{2}, p_{3}$ are all $Y \cup\{v\}$-complete, and every other neighbour of $v$ in $C$ has the same wheel-parity as $p_{1}$, or

- we can number the vertices of $C$ as $p_{1}, \ldots, p_{n}$ in order, such that $p_{1}, p_{2}, p_{3}$ are all $Y$-complete, and there is a path $p_{1}-f_{1}-\cdots-f_{k}-p_{3}$ with interior in $F$, such that there are no edges between $\left\{f_{1}, \ldots, f_{k}\right\}$ and $\left\{p_{4}, \ldots, p_{n}\right\}$.

Proof. We may assume that $F$ is minimal. If $|F|=1$ then the result follows from 16.1, so we assume $|F| \geq 2$.

(1) If there do not exist nonadjacent vertices in $X$ with different wheel-parity, then the theorem holds.

For there exist vertices in $X$ with different wheel-parity, which are therefore adjacent; say $p_{1}, p_{2}$, where $C$ has vertices $p_{1}, \ldots, p_{n}$ in order. So $p_{1}, p_{2}$ are both $Y$-complete, since they have different wheel-parity. There is a third attachment of $F$, since there are two that are nonadjacent, say $p_{i}$ where $3 \leq i \leq n$. Since $p_{1}, p_{2}$ have different wheel-parity, we may assume that $p_{2}, p_{i}$ have different wheel-parity; and therefore $p_{2}, p_{i}$ are adjacent, that is, $i=3$, and $p_{3}$ is $Y$-complete. Suppose $F$ has a fourth attachment $p_{j}$ say, where $4 \leq j \leq n$. From the symmetry we may assume $j \neq n$; and so $p_{j}$ is nonadjacent to both $p_{1}, p_{2}$, and one of these has different wheel-parity from $p_{j}$, a contradiction. So $p_{1}, p_{2}, p_{3}$ are the only attachments of $F$, and then the theorem holds. This proves (1).

From (1) we may assume there are nonadjacent vertices in $X$ with opposite wheel-parity, say $x_{1}, x_{2}$, and therefore $F$ is the interior of a path between $x_{1}, x_{2}$, from the minimality of $F$. Let $C$ have vertices $p_{1}, \ldots, p_{n}$ in order; then we may assume that there exists $m$ with $3 \leq m \leq n-1$ such that $p_{1}, p_{m}$ have opposite wheel-parity, and there is a path $p_{1}-f_{1}-\cdots-f_{k}-p_{m}$ where $F=\left\{f_{1}, \ldots, f_{k}\right\}$. Let $X_{1}$ be the set of attachments in $C$ of $F \backslash\left\{f_{k}\right\}$, and $X_{2}$ the set of attachments of $F \backslash\left\{f_{1}\right\}$. From the minimality of $F$, for $i=1,2$ either all members of $X_{i}$ have the same wheel-parity, or there are at most two members of $X_{i}$, adjacent if there are two. Since $k \geq 2$ it follows that $X_{1} \cup X_{2}=X$. 
(2) $X_{1}$ and $X_{2}$ do not both have members of opposite wheel-parity.

For suppose they do; then $X_{1}, X_{2}$ both consist of exactly two adjacent vertices of opposite wheelparity, say $X_{1}=\left\{p_{1}, p_{2}\right\}$ and $X_{2}=\left\{p_{m^{\prime}}, p_{m^{\prime}+1}\right\}$. So $p_{1}, p_{2}, p_{m^{\prime}}, p_{m^{\prime}+1}$ are all $Y$-complete, and all distinct since two of them are nonadjacent and of opposite wheel-parity. So the only edges between $F$ and $\left\{p_{1}, p_{2}\right\}$ are incident with $f_{1}$, and similarly for $f_{k}$. But then $G$ contains a long prism since $n \geq 6$, a contradiction. This proves (2).

(3) If $X_{1}$ has members of opposite wheel-parity then the theorem holds.

For assume $X_{1}$ has members of opposite wheel-parity. Then we may assume its only members are $p_{1}, p_{2}$, and they are both $Y$-complete. From (2) we may assume that all members of $X_{2}$ have the same wheel-parity as $p_{2}$. In particular, $p_{1}$ has no neighbour in $F \backslash\left\{f_{1}\right\}$. So the only edges between $F$ and $C$ are $f_{1} p_{1}$, edges incident with $p_{2}$, and edges incident with $f_{k}$. Suppose that $p_{2}$ also has no neighbour in $F \backslash\left\{f_{1}\right\}$, and therefore $p_{2}$ is adjacent to $f_{1}$. If $f_{k}$ has a unique neighbour $x$ in $C$, then $x$ can be linked onto the triangle $\left\{p_{1}, p_{2}, f_{1}\right\}$; if $f_{k}$ has two nonadjacent neighbours in $C$ then $f_{k}$ can be linked onto the same triangle; and if it has exactly two neighbours and they are adjacent, then $G$ contains a long prism, in each case a contradiction. So $p_{2}$ has a neighbour in $F \backslash\left\{f_{1}\right\}$. Let $R_{1}$ be the path $p_{1}-f_{1}-\cdots-f_{k}$, and let $R_{2}$ be the path from $p_{2}$ to $f_{k}$ with interior in $F \backslash\left\{f_{1}\right\}$. Then $p_{1}$ has no neighbours in $R_{2} \backslash p_{2}$. Let $Q_{1}$ be the path from $f_{k}$ to $p_{n}$ with interior in $C \backslash p_{1}$. Now $p_{1}-R_{1}-f_{k}-Q_{1}-p_{n}-p_{1}$ is a hole, so $R_{1}$ and $Q_{1}$ have lengths of opposite parity; and since this hole contains an odd number of $Y$-complete edges (since all neighbours of $f_{k}$ have wheel-parity opposite from that of $p_{1}$ ) it follows from 2.3 that it contains exactly one such edge and only two $Y$-complete vertices. Since $p_{1}$ is $Y$-complete, the other is therefore $p_{n}$. The path $p_{2}-R_{2}-f_{k}-Q_{1}-p_{n}$ is between $Y$-complete vertices, and no internal vertex is $Y$-complete, and the $Y$-complete vertex $p_{1}$ has no neighbour in its interior; so it is even by 2.2 , that is, $R_{1}, R_{2}$ have opposite parity. Now there is a $Y$-complete vertex in $\left\{p_{4}, \ldots, p_{n-1}\right\}$; for there are two disjoint $Y$-complete edges in $C$, and an even number of $Y$-complete edges in $C$. Let $p_{s}$ be such a vertex, where $4 \leq s \leq n-1$. We claim that $f_{k}$ has a neighbour in $\left\{p_{4}, \ldots, p_{n-1}\right\}$. For if not, then since $X \neq\left\{p_{n}, p_{1}, p_{2}\right\}$ (because there are nonadjacent vertices in $X$ of opposite wheel-parity), it follows that $f_{k}$ is adjacent to $p_{3}$. Since $p_{s}$ is not in $Q_{1}$, it follows that $p_{3}$ is not in $Q_{1}$, and so $f_{k}$ has another neighbour, which must be $p_{n}$; but then $f_{k}-p_{3}-p_{4^{-}} \cdots-p_{n^{-}} f_{k}$ is an odd hole. So $f_{k}$ has a neighbour in $\left\{p_{4}, \ldots, p_{n-1}\right\}$; and therefore there is a path $Q_{2}$ from $f_{k}$ to some $x$, such that $x$ is the unique $Y$-complete vertex in $Q_{2}$, and $V\left(Q_{2} \backslash\left\{f_{k}\right\}\right) \subseteq\left\{p_{4}, \ldots, p_{n-1}\right\}$. Now the path $p_{2}-R_{2}-f_{k}-Q_{2}$ has both ends $Y$-complete, and no internal vertex $Y$-complete, and the $Y$-complete vertex $p_{1}$ has no neighbour in its interior, so it is even by 2.2. Therefore the path $p_{1}-R_{1}-f_{k}-Q_{2}$ is odd, since $R_{1}, R_{2}$ have opposite parity; and again its ends are $Y$-complete and its internal vertices are not. So it has length 3 , by 13.6, and so $k=2$; and every $Y$-complete vertex is adjacent to one of $f_{1}, f_{2}$. Consequently there is no $Y$-complete vertex in $C$ different from $p_{1}$ with the same wheel-parity as $p_{1}$, a contradiction. This proves (3).

From (3) we may assume that all members of $X_{1}$ have the same wheel-parity, and all members of $X_{2}$ have the opposite wheel-parity. It follows that $X_{1} \cap X_{2}=\emptyset$, and so there are no edges between the interior of $F$ and $C$. So $X_{1}$ is the set of neighbours of $f_{1}$ in $C$, and $X_{2}$ is the set of neighbours of $f_{k}$ in $C$. 
(4) At least one of $f_{1}, f_{k}$ has only one neighbour in $C$.

For assume they both have at least two. Then there are disjoint paths $Q, R$ of $C$, both containing neighbours of both $f_{1}, f_{k}$. Choose $Q, R$ minimal, and let $Q$ have ends $q_{1}, q_{2}$; then from the minimality of $Q, q_{1}$ is the unique neighbour of one of $f_{1}, f_{k}$ in $Q$, and $q_{2}$ is the unique neighbour of the other. Let $f_{1} q_{1}$ and $f_{k} q_{2}$ be edges say. Similarly let $R$ have ends $r_{1}, r_{2}$, where $f_{1} r_{1}, f_{k} r_{2}$ are edges. Since $q_{1}, q_{2}$ have opposite wheel-parity, it follows that there are an odd number of $Y$-complete edges in the hole $f_{1^{-}} \cdots-f_{k^{-}} q_{2}-Q-q_{1}-f_{1}$; so by 2.3 there is exactly one, and just two $Y$-complete vertices. If there are no edges between $Q$ and $R$ this contradicts 15.3 , applied to the cycle $f_{1}-q_{1}-Q-q_{2}-f_{k^{-}}-r_{2}-R-r_{1^{-}} f_{1}$. Since $Q, R$ are disjoint subpaths of $C$, all the edges between them join their ends; so we may assume that $q_{1}$ is adjacent to one of $r_{1}, r_{2}$. From the hole $f_{1^{-}} \cdots-f_{k^{-}} q_{2}-Q-q_{1}-f_{1}$ it follows that $Q$ has parity $k-1$, and similarly so does $R$. Suppose first that $q_{1}$ is adjacent to $r_{1}$. Since $q_{1}-Q-q_{2}-f_{k}-r_{2}-R-r_{1}-q_{1}$ is not an odd hole, it follows that $q_{2}$ is adjacent to $r_{2}$, and hence $G$ contains a long prism, since $C$ has length $\geq 6$, a contradiction. So $q_{1}$ is adjacent to $r_{2}$. Since $q_{1}$ is a neighbour of $f_{1}$ and $r_{2}$ of $f_{k}$, it follows that $q_{1}, r_{2}$ have opposite wheel-parity, and since they are adjacent, they are both $Y$-complete. Let $q^{\prime}$ be the neighbour of $q_{1}$ in $Q$, let $Q^{\prime}=Q \backslash q_{1}$, let $r^{\prime}$ be the neighbour of $r_{2}$ in $R$, and let $R^{\prime}=R \backslash r_{2}$. Since in the hole $f_{1^{-}} \cdots-f_{k^{-}} q_{2}-Q-q_{1}-f_{1}$ there are only two $Y$-complete vertices and they are adjacent, it follows that the second is $q^{\prime}$, and similarly $r^{\prime}$ is $Y$-complete. If $q_{2}$ is adjacent to $r_{1}$ then not both $q_{2}, r_{1}$ are $Y$-complete since $C$ has length $\geq 6$; and so there are exactly three $Y$-complete edges in $C$, contrary to 2.3. It follows that $q_{2}$ is not adjacent to $r_{1}$. From the hole $q_{1}-Q-q_{2}-f_{k}-r_{2}-q_{1}$ it follows that $Q$ has odd length, and therefore so does $R$ and $k$ is even. But then the path $q^{\prime}-Q^{\prime}-q_{2}-f_{k^{-}} \cdots-f_{1}-r_{1}-R^{\prime}-r^{\prime}$ has odd length, its ends are $Y$-complete and its internal vertices are not, and so by 13.6 it has length 3 ; that is, $Q, R$ have length 1 and $k=2$. Hence the path $r_{1}-f_{1^{-}} f_{2}-q_{2}$ is odd, its ends are $Y$-complete, and its internal vertices are not, so every $Y$-complete vertex is adjacent to one of $f_{1}, f_{2}$. Let $a b, a^{\prime} b^{\prime}$ be two $Y$-complete edges of $C$, disjoint and such that there are no edges from $\{a, b\}$ to $\left\{a^{\prime}, b^{\prime}\right\}$. Then each of $a, b, a^{\prime}, b^{\prime}$ is adjacent to one of $f_{1}, f_{2}$, and since all neighbours of $f_{1}$ in $C$ have opposite wheel-parity from all neighbours of $f_{2}$ in $C$, we may assume that $a, a^{\prime}$ are adjacent to $f_{1}$ and $b, b^{\prime}$ to $f_{2}$. But this contradicts 15.3 , applied to the cycle $a-f_{1}-a^{\prime}-b^{\prime}-f_{2}-b-a$. This proves (4).

From (4) we may assume that $X_{1}$ has only one member, say $p_{1}$. Choose $i, j$ with $2 \leq i, j \leq n$, such that $p_{i}, p_{j}$ are adjacent to $f_{k}$, with $i$ minimum and $j$ maximum. From the hole $p_{1}-f_{1^{-}} \cdots-f_{k}-p_{i}-p_{i-1} \cdots-p_{1}$

( $=H_{1}$ say) we deduce that $i, k$ have the same parity, and from the hole $p_{1}-f_{1^{-}} \cdots-f_{k}-p_{j}-p_{j+1^{-}} \cdots-p_{n}-p_{1}$ ( $=H_{2}$ say) that $j, k$ have the same parity. (So either $p_{i}=p_{j}$ or $p_{i}, p_{j}$ are nonadjacent.) Since $p_{1}, p_{i}$ have different wheel-parity, and so do $p_{1}, p_{j}$, there is an odd number of $Y$-complete edges in each of $H_{1}, H_{2}$; and therefore there is exactly one $Y$-complete edge and exactly two $Y$-complete vertices in each of the holes, by 2.3. Suppose that $i=j$. Then there are only two $Y$-complete edges in $C$, and therefore they are disjoint, and $p_{1}, p_{i}$ are not $Y$-complete (since $H_{1}, H_{2}$ both have only two $Y$-complete vertices), contrary to 15.3 applied to $C$. So $j>i$, and hence $j \geq i+2$. If $p_{1}$ is not $Y$-complete, then the $Y$-complete edge in $H_{1}$ is disjoint from the path $p_{1}-f_{1}-\cdots-f_{k}$, and so is the one in $H_{2}$; but this contradicts 15.3 applied to the hole $p_{1^{-}} \cdots-p_{i^{-}} f_{k^{-}}-p_{j^{-}} \cdots-p_{n}-p_{1}$. So $p_{1}$ is $Y$-complete. Since $H_{1}$ contains only two $Y$-complete vertices and they are adjacent, the other is $p_{2}$, and similarly $p_{n}$ is $Y$-complete.

(5) $f_{k}$ has no neighbour in $\left\{p_{3}, \ldots, p_{j-2}\right\}$. 
For assume it does. We claim there is also a $Y$-complete vertex in this set; for otherwise the only $Y$ complete vertices in $C$ are $p_{n}, p_{1}, p_{2}$ and possibly $p_{j-1}$, which is impossible since there are two disjoint $Y$-complete edges and an even number of $Y$-complete edges in $C$. Hence there is a path $P$ say from $f_{k}$ to some $x$ such that $x$ is the unique $Y$-complete vertex in $P$ and $V\left(P \backslash f_{k}\right) \subseteq\left\{p_{3}, \ldots, p_{j-2}\right\}$. The path $p_{n}-p_{n-1^{-}} \cdots-p_{j}-f_{k}-P$-x is even, since its ends are $Y$-complete, no internal vertex is $Y$-complete, and the $Y$-complete vertex $p_{1}$ has no neighbour in its interior. The path $p_{1}-f_{1}-\cdots-f_{k}-P-x$ is therefore odd (since $k, j$ have opposite parity), and also its ends are $Y$-complete and no internal vertex is $Y$-complete; so it has length 3 by 13.6, and hence $k=2$ and every $Y$-complete vertex is adjacent to one of $f_{1}, f_{2}$, by 2.2. So there is no $Y$-complete vertex in $C \backslash p_{1}$ with the same wheel-parity as $p_{1}$, a contradiction. This proves (5).

Since $f_{k}$ is adjacent to $p_{i}$, and $i<j$ and $j-i$ is even, it follows from (5) that $i=2$, and similarly $f_{k}$ has no neighbours in $\left\{p_{i+2}, \ldots, p_{n-1}\right\}$ and $j=n$. So $f_{k}$ has no neighbours in

$$
\left\{p_{3}, \ldots, p_{j-2}\right\} \cup\left\{p_{i+2}, \ldots, p_{n-1}\right\}=\left\{p_{3}, \ldots, p_{n-1}\right\},
$$

and therefore $p_{2}, p_{n}$ are its only neighbours, contradicting that there are nonadjacent vertices in $X$ of opposite wheel-parity. This proves 16.2.

The main result of this section is 1.8 .7 , which we restate, the following.

16.3 Let $G \in \mathcal{F}_{6}$. If there is an odd wheel in $G$ then $G$ admits a balanced skew partition. In particular, every recalcitrant graph belongs to $\mathcal{F}_{7}$.

Proof. Suppose $(C, Y)$ is an odd wheel with $Y$ maximal, and subject to that, such that the number of $Y$-complete edges in $C$ is minimum. (We refer to these conditions as the "optimality" of $(C, Y)$.)

(1) There is no vertex $v \in V(G) \backslash(V(C) \cup Y)$ such that $v$ is not $Y$-complete and has nonadjacent neighbours in $C$ of opposite wheel-parity.

Suppose there is such a vertex $v$. Suppose first that there is an odd $Y \cup\{v\}$-segment in $C$. From the maximality of $Y,(C, Y \cup\{v\})$ is therefore not a wheel, and so there is a unique $Y \cup\{v\}$-complete edge in $C$. By 2.10, either $v$ has only two neighbours in $C$, or some vertex of $Y$ has only three, in either case a contradiction. So there is no odd $Y \cup\{v\}$-segment in $C$. Define a "line" to be a maximal subpath of $C$ with no internal vertex adjacent to $v$. It follows that every edge of $C$ is in a unique line. Let $C$ have vertices $p_{1}, \ldots, p_{n}$ in order, and let $S$ be an odd $Y$-segment.

Since there are no odd $Y \cup\{v\}$-segments, it follows that an even number of edges of $S$ are $Y \cup\{v\}$-complete. Hence an odd number are not, and therefore there is a line $L$ containing an odd number of edges of $S$ that are not $Y \cup\{v\}$-complete. In particular it contains at least one edge that is $Y$-complete and not $Y \cup\{v\}$-complete, so $L$ has length $>1$. Let the ends of $L$ be $p, q$. By 16.1, $p$ and $q$ have the same wheel-parity with respect to $(C, Y)$, and so $L$ contains an odd number of edges of some other $Y$-segment $S^{\prime} \neq S$. In particular, there are two disjoint $Y$-complete edges in the hole $v-p-L-q-v$ ( $=H$ say); so $H$ has length $\geq 6$ (because $v$ is not $Y$-complete) and so $(H, Y)$ is a wheel. Moreover it is an odd wheel, for it contains an odd number of edges of $S$, and those edges form either one or two $Y$-segments in $H$, and one of these segments is odd. Since there is a $Y \cup\{v\}$-complete 
edge in $C$ (by 16.1, since $v$ has neighbours in $C$ of opposite wheel-parity) which therefore does not belong to $L$, this contradicts the optimality of $(C, Y)$. This proves (1).

Since $(C, Y)$ is an odd wheel, $C$ has at least two segments, and therefore there are vertices $u, v$ in $C$ with different wheel-parity and neither of them $Y$-complete. Let $X$ be the set of all $Y$-complete vertices in $V(G)$. Then $|X|>1$, since $|X \cap V(C)| \geq 4$; so by 15.2 , we may assume that $V(G) \backslash(X \cup Y)$ is nonempty and connected ( $=Z$ say), and every vertex in $X$ has a neighbour in it, for otherwise $G$ admits a balanced skew partition and the theorem holds. In particular $u, v \in Z$, so there is a minimal connected subset $F$ of $Z$ such that there are two vertices of $C \backslash X$ (say $p, q$ ) of opposite wheel-parity, both with neighbours in $F$. Since $p, q$ have opposite wheel-parity and are not $Y$-complete, they are not adjacent. From the minimality of $F, F$ is a path, and no vertex of $F$ is in $C$. By 16.2 and (1), there is a 3 -vertex path $p_{1}-p_{2}-p_{3}$ in $C$, all $Y$-complete, and a path $p_{1}-f_{1}-\cdots-f_{k}-p_{3}$ with interior in $F$, such that there no edges between $\left\{f_{1}, \ldots, f_{k}\right\}$ and $\left\{p_{4}, \ldots, p_{n}\right\}$. But then $C \backslash p_{2}$ can be completed to a hole $C^{\prime}$ say, via $p_{1}-f_{1^{-}} \cdots-f_{k^{-}} p_{3}$; and $C^{\prime}$ has length $\geq 6$. For every odd segment $S$ of $(C, Y)$, either it contained both or neither of the edges $p_{1} p_{2}, p_{2} p_{3}$; and so in either case an odd number of edges of $S$ belong to $C^{\prime}$. Since $(C, Y)$ has an odd segment and there are an even number of $Y$-complete edges in $C$, it has at least two odd segments. It follows that there are two disjoint $Y$-complete edges in $C^{\prime}$, and so $\left(C^{\prime}, Y\right)$ is a wheel. Since an odd number of edges of the odd segment $S$ belong to $C^{\prime}$, it follows that $\left(C^{\prime}, Y\right)$ is an odd wheel, contrary to the optimality of $(C, Y)$. This proves 16.3 .

\section{Another extension of the Roussel-Rubio lemma}

A "pseudowheel" is a variant of an odd wheel, defined in the next section, and we want to show that Berge graphs containing pseudowheels and nothing better admits balanced skew partitions. The main result of this section is a lemma about graphs in $\mathcal{F}_{7}$, that will be used in when we handle pseudowheels in section 18 .

Let $\left\{a_{1}, a_{2}, a_{3}\right\}$ be a triangle in $G$. A reflection of this triangle is another triangle $\left\{b_{1}, b_{2}, b_{3}\right\}$ of $G$, disjoint from the first, such that $a_{1} b_{1}, a_{2} b_{2}, a_{3} b_{3}$ are edges, and these are the only edges between the two triangles. Hence these six vertices induce a prism. A subset $F$ of $V(G)$ is said to catch the triangle $\left\{a_{1}, a_{2}, a_{3}\right\}$ if it is connected and disjoint from that triangle and $a_{1}, a_{2}, a_{3}$ all have neighbours in $F$. We begin with the following extremely useful little fact.

17.1 Let $A$ be a triangle in a graph $G \in \mathcal{F}_{7}$, and let $F \subseteq V(G) \backslash A$ catch $A$. Then either $F$ contains a reflection of $A$, or some vertex of $F$ has $\geq 2$ neighbours in $A$.

Proof. Suppose not, and choose $F$ minimal such that it catches $A$. Let $A=\left\{a_{1}, a_{2}, a_{3}\right\}$ say, and for $i=1,2,3$, let $B_{i}$ be the set of neighbours of $a_{i}$ in $F$. Thus the three sets $B_{1}, B_{2}, B_{3}$ are pairwise disjoint and nonempty.

(1) There is no path in $F$ meeting all of $B_{1}, B_{2}, B_{3}$.

For assume there is, and choose it minimal. So then we may assume there is a path $P$ from $b_{1} \in B_{1}$ to $b_{2} \in B_{2}$, such that some vertex of $P$ is in $B_{3}$, and for $i=1,2, b_{i}$ is the only vertex of $P$ in $B_{i}$. Since $B_{3}$ is disjoint from $B_{1} \cup B_{2}$, every vertex of $B_{3}$ in $P$ is an internal vertex of $P$; and so $P$ has 
length $\geq 2$. But then $\left(C,\left\{a_{3}\right\}\right)$ is an odd wheel, where $C$ is the hole $a_{1}-b_{1}-P-b_{2}-a_{2}-a_{1}$, contrary to $G \in \mathcal{F}_{7}$. This proves (1).

Choose $b_{1} \in F$ such that $F \backslash\left\{b_{1}\right\}$ is connected; then from the minimality of $F, F \backslash\left\{b_{1}\right\}$ does not catch $A$, and so we may assume that $B_{1}=\left\{b_{1}\right\}$. Since $F$ is connected and $|F| \geq 2$, there is a second vertex $b_{2} \neq b_{1}$ in $F$ such that $F \backslash\left\{b_{2}\right\}$ is connected, and so similarly we may assume $B_{2}=\left\{b_{2}\right\}$. Let $P$ be a path in $F$ between $b_{1}, b_{2}$. By (1) no vertex of $P$ is in $B_{3}$, so $F$ contains a connected subset $F^{\prime}$ including $V(P)$ which contains exactly one vertex of $B_{3}$. From the minimality of $F,\left|B_{3}\right|=1$; let $B_{3}=\left\{b_{3}\right\}$ say. Let $Q$ be a minimal path in $F$ such that $b_{3} \in V(Q)$ and some vertex of $P$ has a neighbour in $Q$. From the minimality of $Q$ it follows that $Q$ is vertex-disjoint from $P$, and $Q$ has ends $b_{3}, x$ say, where $x$ is the unique vertex of $Q$ with neighbours in $P$. From the minimality of $F, x$ either has one neighbour in $P$, or just two neighbours and they are adjacent; for if it has two nonadjacent neighbours, any vertex of $P$ between them could be deleted from $F$, contrary to the minimality of $F$. If $x$ has just one neighbour ( $y$ say) in $P$, then $y$ can be linked onto the triangle $A$, contrary to 2.4; so it has two adjacent neighbours. Since $G$ does not contain a long prism it follows that $Q$ has length 0 and $P$ has length 1 , and so $F$ contains a reflection of $A$, as required. This proves 17.1 .

We did not use the full strength of $G \in \mathcal{F}_{7}$ in proving 17.1; we just used that there were no odd wheels with hubs of cardinality 1. This suggests that there should be some generalization of 17.1 whose proof does use the full strength of the hypothesis that there are no odd wheels, and that is true, but not easy - it will be a consequence of the main result of this section.

Before we start on that, let us give a strengthening of 2.10 for graphs in $\mathcal{F}_{7}$.

17.2 Let $G \in \mathcal{F}_{7}$, and let $F, Y \subseteq V(G)$ be disjoint, such that $F$ is connected and $Y$ is anticonnected. Let $a_{0}, b_{0} \in V(G) \backslash(F \cup Y)$ and $a, b \in F$ such that $a-a_{0}-b_{0}-b$ is a 3-edge path in $G$. Suppose that:

- $a_{0}, b_{0}$ are both $Y$-complete, and $a, b$ are not $Y$-complete,

- the only neighbours of $a_{0}, b_{0}$ in $F$ are $a$ and $b$ respectively,

- $F \backslash\{a\}$ and $F \backslash\{b\}$ are both connected.

Then either:

1. there is a vertex in $Y$ with no neighbour in $F$, or

2. there are two nonadjacent vertices $y_{1}, y_{2} \in Y$, such that $a$ is the only neighbour of $y_{1}$ in $F$, and $b$ is the only neighbour of $y_{2}$ in $F$.

Proof. We may assume that every vertex in $Y$ has a neighbour in $F$, for otherwise statement 1 of the theorem holds.

(1) There exist nonadjacent $y_{1}, y_{2}$ in $Y$, such that $y_{1}$ is adjacent to a and not $b$, and $y_{2}$ is adjacent to $b$ and not $a$.

For choose a path $P$ between $a$ and $b$ with $V(P) \subseteq F$. Then the hole $a_{0}-a-P-b-b_{0}-a_{0}(=C$, say) has length $\geq 6$. If there are any $Y$-complete vertices in $P$, then they belong to the interior of $P$ since $a, b$ 
are not $Y$-complete, and there is an odd number of $Y$-complete edges in $P$, by 2.3 ; but then $(C, Y)$ is an odd wheel (the path $a_{0}-b_{0}$ is an odd $Y$-segment), a contradiction. So there are no $Y$-complete vertices in $P$. By 2.10 applied to $C, Y$ contains either a hat or a leap. Suppose first it contains a hat, that is, there is a vertex $y \in Y$ with no neighbour in $P$. By the assumption above, $y$ has a neighbour in $F$. Consequently $F$ catches the triangle $\left\{a_{0}, b_{0}, y\right\}$. But $y$ is not adjacent to $a$ or $b$ since it has no neighbour in $P$, and $a$ is the only vertex in $F$ adjacent to $a_{0}$, and the same for $b, b_{0}$; and $a, b$ are nonadjacent, so $F$ contains no reflection of the triangle. This contradicts 17.1. Hence there is no such $y$, and so there is a leap. This proves (1).

(2) There is no path in $F$ between $a$ and $b$ such that $y_{1}$ or $y_{2}$ has a neighbour in its interior.

For suppose there is such a path, $P^{\prime}$ say. Then the set $\left\{y_{1}, y_{2}\right\}$ contains neither a leap not a hat for the hole $a_{0}-a-P^{\prime}-b-b_{0}-a_{0}$ ( $=C$ say), and so by 2.10 there is a vertex in $P$ adjacent to both $y_{1}, y_{2}$. By 2.3 there is an even number of $\left\{y_{1}, y_{2}\right\}$-complete edges in this hole, and since $a, b$ are not $\left\{y_{1}, y_{2}\right\}$-complete, $\left(C,\left\{y_{1}, y_{2}\right\}\right)$ is an odd wheel, a contradiction. This proves (2).

Now if neither of $y_{1}, y_{2}$ has any more neighbours in $F$ then statement 2 of the theorem holds; so we assume at least one of them has another neighbour in $F$. Since $F \backslash\{a\}, F \backslash\{b\}$ are both connected, there is a connected subset $F^{\prime}$ of $F \backslash\{a, b\}$, such that both $a$ and $b$ have neighbours in $F^{\prime}$, and at least one of $y_{1}, y_{2}$ has a neighbour in $F^{\prime}$. Choose $F^{\prime}$ minimal with these properties. At least one of $y_{1}, y_{2}$ has a neighbour (say $x$ ) in $F^{\prime}$. We claim that $F^{\prime} \backslash\{x\}$ is connected. For if not, let $L$ be a component of it, and $M$ the union of the other components. From the minimality of $F$, not both $a, b$ have neighbours in $L \cup\{x\}$, and not both have neighbours in $M \cup\{x\}$; so we may assume all neighbours of $a$ in $F^{\prime}$ are in $L$, and all neighbours of $b$ are in $M$. But then there is a path from $a$ to $b$ with interior in $F$ and with $x$ in its interior, contrary to (2). This proves that $F^{\prime} \backslash\{x\}$ is connected. There is a path from $a$ to $b$ with interior in $F^{\prime}$, and $x$ is not in it, by (2), and it has length $>1$ since $a, b$ are nonadjacent. So $a, b$ both have neighbours in $F^{\prime} \backslash\{x\}$. From the minimality of $F^{\prime}, y_{1}$ and $y_{2}$ both have no neighbours in $F^{\prime} \backslash\{x\}$. We claim that $x$ is adjacent to both $y_{1}$ and $y_{2}$. For it is adjacent to at least one, say $y_{1}$; let $Q$ be a path from $x$ to $b$ with interior in $F^{\prime}$. Then $y_{1}-x-Q-b$ is a path, since $y_{1}$ has no more neighbours in $F^{\prime}$. Since $b_{0}-y_{1}-x-Q-b-b_{0}$ is a hole it follows that $Q$ is odd. Therefore $a_{0}-y_{1}-x-Q-b-y_{2}-a_{0}$ is not a hole, and so $y_{2}$ has neighbours in $Q$. Since it has no neighbours in $F^{\prime} \backslash\{x\}$, this proves our claim that $x$ is adjacent to both $y_{1}, y_{2}$.

With $Q$ as before, and therefore odd, it follows that $y_{2}-x-Q-b-y_{2}$ is not a hole, and therefore $Q$ has length 1 , that is, $x$ is adjacent to $b$. Similarly $x$ is adjacent to $a$; but then $x-a-a_{0}-b_{0}-b-x$ is an odd hole, a contradiction. This proves 17.2.

The following is a variant of 17.2 , not so symmetrical, but more useful.

17.3 Let $G \in \mathcal{F}_{7}$, and let $F, Y \subseteq V(G)$ be disjoint, such that $F$ is connected and $Y$ is anticonnected. Let $a_{0}, b_{0} \in V(G) \backslash(F \cup Y)$ and $a, b \in F$ such that $a-a_{0}-b_{0}-b$ is a 3-edge path in $G$. Suppose that:

- $a_{0}, b_{0}$ are both $Y$-complete, and $a, b$ are not $Y$-complete,

- the only neighbours of $a_{0}, b_{0}$ in $F$ are $a$ and $b$ respectively,

- $F \backslash\{a\}$ is connected.

Then there is a vertex $y \in Y$ with no neighbour in $F \backslash\{a\}$. 
Proof. If $F \backslash\{b\}$ is connected, the result follows from 17.2. So assume it is not, and let $F_{1}^{\prime}$ be the component of $F \backslash\{b\}$ that contains $a$, and $F_{2}^{\prime}$ the union of all the other components. For $i=1,2$ let $F_{i}=F_{i}^{\prime} \cup\{b\}$. Then $F_{1} \backslash\{a\}, F_{1} \backslash\{b\}$ are both connected, so by 17.2 either there exists $y \in Y$ with no neighbour in $F_{1}$, or there exist nonadjacent $y_{1}, y_{2} \in Y$ with no neighbours in $F_{1}$ except $a, b$ respectively. Suppose the first. If $y$ has a neighbour in $F_{2}$ then $b$ can be linked onto the triangle $\left\{y, a_{0}, b_{0}\right\}$, a contradiction to 2.4; and if not then $y$ satisfies the theorem. Now suppose the second. If $y_{1}$ has neighbours in $F_{2}$ then $(F \backslash\{a\}) \cup\left\{y_{2}\right\}$ catches the triangle $\left\{a, a_{0}, y_{1}\right\}$; the only neighbours of $a, a_{0}, y_{1}$ belong to the disjoint sets $F_{1}^{\prime},\left\{y_{2}\right\}, F_{2}^{\prime}$; and there is no reflection since there are no edges between $y_{2}$ and $F_{1}^{\prime}$, contrary to 17.1. So $y_{1}$ has no neighbours in $F_{2}$. This proves 17.3.

The next result is just a technical lemma for use in proving the main result of this section, which is 17.5 .

17.4 Let $G \in \mathcal{F}_{7}$ and let $P$ be a path in $G$ with length $>1$, with vertices $p_{1}, \ldots, p_{n}$ in order. Let $X, Y \subseteq V(G) \backslash V(P)$ be anticonnected sets, such that $X \cup Y$ is anticonnected, $p_{1}$ is $X$-complete, and $p_{n}$ is the unique $Y$-complete vertex in $P$. (Note that $X, Y$ need not be disjoint.) Let $z \in$ $V(G) \backslash(X \cup Y \cup V(P))$, complete to $X \cup Y$ and with no neighbours in $P$. Assume that $p_{n}$ is not $X$-complete. Let $p_{n}-x_{1}-\cdots-x_{k}-y$ be an antipath with interior in $X$ from $p_{n}$ to some $y \in Y$. Then $p_{n-1}$ is nonadjacent to $x_{1}$.

Proof. Let $F=\left\{p_{n-1}, x_{1}, \ldots, x_{k}\right\} \cup Y$. Since $p_{n-1}$ is not $Y$-complete it follows that $F$ is anticonnected, and both $F \backslash\left\{p_{n-1}\right\}, F \backslash\left\{x_{1}\right\}$ are anticonnected. The only nonneighbour of $z$ in $F$ is $p_{n-1}$, and the only nonneighbour of $p_{n}$ in $F$ is $x_{1}$; and we may assume that $p_{n-1}$ is adjacent to $x_{1}$. Now $p_{n-1}-z-p_{n}-x_{1}$ is a path in $\bar{G}$, and $F$ is connected in $\bar{G}$, and $\left\{p_{1}, \ldots, p_{n-2}\right\}$ is anticonnected in $\bar{G}$. Also, $z$ and $p_{n}$ are $\left\{p_{1}, \ldots, p_{n-2}\right\}$-complete in $\bar{G}$, and $p_{n-1}, x_{1}$ are not. We may therefore apply 17.2 in $\bar{G}$, and deduce that there is a vertex in $\left\{p_{1}, \ldots, p_{n-2}\right\}$ which is complete (in $G$ ) to $F \backslash\left\{p_{n-1}\right\}$. But then this vertex is $Y$-complete, a contradiction. This proves 17.4 .

We gave in 2.9 an extension of the Roussel-Rubio lemma to two anticonnected sets instead of one (we haven't had much use of that theorem yet, but its time is coming.) In that extension the two sets had to be complete to each other. Now we prove a similar result where the two sets are not complete to each other. Incidentally, unlike 2.9 , what we are going to prove here is not true for general Berge graphs — we need the hypothesis that $G \in \mathcal{F}_{7}$.

17.5 Let $G \in \mathcal{F}_{7}$ and let $P$ be an odd path in $G$ with length $>1$, with vertices $p_{1}, \ldots, p_{n}$ in order. Let $X, Y \subseteq V(G) \backslash V(P)$ be anticonnected sets, such that $X \cup Y$ is anticonnected, $p_{1}$ is $X$ complete, and $p_{n}$ is the unique $Y$-complete vertex in $P$. (Note that $X, Y$ need not be disjoint.) Let $z \in V(G) \backslash(X \cup Y \cup V(P))$, complete to $X \cup Y$ and with no neighbours in $P$. Then an odd number of edges of $P$ are $X$-complete.

Proof. If possible choose a counterexample $P, X, Y$ such that

1. $P$ is minimal

2. subject to condition $1, X \cup Y$ is minimal, and

3. subject to conditions 1 and $2,|X|+|Y|$ is minimum. 
We refer to this property as the "optimality" of $P, X, Y$.

\section{(1) No vertex of $P \backslash p_{1}$ is $X$-complete.}

If $p_{n}$ is $X$-complete, then since $P$ has odd length $>1$, and the $X$-complete vertex $z$ has no neighbour in $P$, it follows from 2.2 and 2.3 that there are an odd number of $X$-complete edges in $P$, and the theorem holds, a contradiction. So $p_{n}$ is not $X$-complete. By $17.4, p_{n-1}$ is not $X$-complete. Since $p_{1}$ is $X$-complete, we can choose $i$ with $1 \leq i \leq n$ maximum such that $p_{i}$ is $X$-complete. So $i \leq n-2$. Since $z$ has no neighbour in the path $p_{1^{-}} \cdots-p_{i}$, if $i$ is even then there is an odd number of $X$-complete edges in this path and hence in $P$, by 2.2 and 2.3. So we may assume that $i$ is odd. Hence the theorem is also false for $X, Y$ and the path $p_{i^{-}} \cdots-p_{n}$. From the optimality of $P, X, Y$ it follows that $i=1$. This proves (1).

In view of (1), there is symmetry between $X$ and $Y$.

(2) Suppose that $x_{1}, x_{2} \in X$ are distinct and such that $X \backslash\left\{x_{i}\right\}$ is anticonnected for $i=1,2$. Then $X \cap Y=\emptyset$, and one of $x_{1}, x_{2}$ is the unique vertex of $X$ with a nonneighbour in $Y$.

For if $\left(X \backslash\left\{x_{i}\right\}\right) \cup Y$ is not anticonnected for some $i$, then $Y$ is disjoint from $X \backslash\left\{x_{i}\right\}$ (since both these sets are anticonnected), and $Y$ is complete to $X \backslash\left\{x_{i}\right\}$; and therefore $x_{i} \notin Y$ (since $x_{i}$ has a nonneighbour in $\left.X \backslash\left\{x_{i}\right\}\right)$, so $X \cap Y=\emptyset$. But then the statement of (2) holds. So we may assume that $\left(X \backslash\left\{x_{i}\right\}\right) \cup Y$ is anticonnected for $i=1,2$. From the optimality of $P, X, Y$ it follows that the theorem holds for $X \backslash\left\{x_{i}\right\}, Y, P$; and so, since $p_{n}$ is the unique $Y$-complete vertex in $P$, it follows that there are an odd number of $X \backslash\left\{x_{i}\right\}$-complete edges in $P$, for $i=1,2$. For $i=1,2$ let $W_{i}$ be the set of $X \backslash\left\{x_{i}\right\}$-complete vertices in $P$. So $W_{1} \cap W_{2}=\left\{p_{1}\right\}$. Let $Q$ be an antipath in $X$ between $x_{1}$ and $x_{2}$. We claim that $Q$ is odd. For since $W_{1} \cap W_{2}=\left\{p_{1}\right\}$, there are nonadjacent vertices $p_{i}, p_{j}$ of $P$, such that $p_{i} \in W_{1} \backslash W_{2}$ and $p_{j} \in W_{2} \backslash W_{1}$; and since $p_{i}-x_{1}-Q-x_{2}-p_{j}-p_{i}$ is an antihole it follows that $Q$ is odd. Let us say a line is a minimal subpath of $P \backslash p_{1}$ meeting both $W_{1}$ and $W_{2}$. So every line has length $\geq 1$, and has one end in $W_{1}$ and the other in $W_{2}$, and has no more vertices in either $W_{1}$ or $W_{2}$. If some line $L$ has odd length $>1$, then the triple $L, X \backslash\left\{x_{1}\right\}, X \backslash\left\{x_{2}\right\}$ is another counterexample to the theorem, contrary to the optimality of $P, X, Y$; and if some line has length 1 , say $p_{i-} p_{i+1}$ where $p_{i} \in W_{1}$, then $z-p_{i}-x_{1}-Q-x_{2}-p_{i+1^{-}} z$ is an odd antihole, a contradiction. Hence every line is even. Choose $i$ minimum with $2 \leq i \leq n$ such that $\left\{p_{2}, \ldots, p_{i}\right\}$ includes a line. (This is possible since both $W_{1}, W_{2}$ meet $P \backslash p_{1}$.) Since all lines have length $\geq 2$ it follows that $i \geq 4$. From the minimality of $i,\left\{p_{2}, \ldots, p_{i-1}\right\}$ does not include a line, and so for some $k \in\{1,2\}$, the path $p_{1}-\cdots-p_{i}$ has both ends $X \backslash\left\{x_{k}\right\}$-complete and no internal vertex $X \backslash\left\{x_{k}\right\}$-complete. But this path has length $\geq 2$, and $z$ has no neighbour in it, so by 2.2 it is even, that is, $i$ is odd. Choose $j$ with $j \geq 2$ maximum such that $\left\{p_{j}, \ldots, p_{n}\right\}$ includes a line. Since every line has length $\geq 2$ it follows that $2 \leq j \leq n-2$. From the maximality of $j$ it follows that for some $k \in\{1,2\}, W_{k} \cap\left\{p_{j}, \ldots, p_{n}\right\}=\left\{p_{j}\right\}$. If the path $p_{j^{-}} \cdots-p_{n}$ has odd length, then $p_{j}, \ldots, p_{n}, X \backslash\left\{x_{k}\right\}, Y$ is a counterexample to the theorem, contrary to the optimality of $P, X, Y$. So $n-j$ is even, and hence $j$ is even. Now $i$ is odd, so if $i \geq j$ then $p_{j^{-}} \cdots-p_{i}$ is an odd line, a contradiction. Hence $i<j$, and $j-i$ is odd. Now the edges $p_{i-1} p_{i}, p_{j} p_{j+1}$ are in lines. Consequently we may choose $r, s$ with $i \leq r<s \leq j$ such that $p_{r}, p_{s} \in W_{1} \cup W_{2}$, and the edges $p_{r-1} p_{r}, p_{s} p_{s+1}$ are in lines, and $s-r$ is odd; and therefore we may choose such $r, s$ with $s-r$ minimum. If there is a line contained in the path $p_{r^{-}} \cdots-p_{s}$, say $p_{h^{-}} \cdots-p_{k}$, then since $k-h$ 
is even, one of the paths $p_{r^{-}} \cdots-p_{h}$ and $p_{k^{-}} \cdots-p_{s}$ is odd, contrary to the minimality of $s-r$. So we may assume that none of $p_{r}, \ldots, p_{s}$ belong to $W_{2}$, and in particular $p_{r}, p_{s} \in W_{1}$. Since $p_{r-1} p_{r}, p_{s} p_{s+1}$ are in lines, and $p_{r}, p_{s} \in W_{1}$, there exist $q, t$ with $2 \leq q<r<s<t \leq n$ such that $p_{q^{-}} \cdots-p_{r}$ and $p_{s} \cdots-p_{t}$ are lines. All lines are even, so $r-q$ and $t-s$ are even, and therefore $t-q$ is odd. Moreover $p_{q}, p_{t} \in W_{2}$, and none of $p_{q+1}, \ldots, p_{t-1}$ belongs to $W_{2}$, and the path $p_{q^{-}} \cdots-p_{t}$ is odd, and $z$ has no neighbour in it, contrary to 2.2 . This proves (2).

(3) There is an antipath $x_{1}-\cdots-x_{s}-y_{1}-\cdots-y_{t}$ such that $s, t>1$ and $X=\left\{x_{1}, \ldots, x_{s}\right\}$, and $Y=$ $\left\{y_{1}, \ldots, y_{t}\right\}$.

For if $|X|=1, X=\{x\}$ say, then $z-x-p_{1}-\cdots-p_{n}$ is an odd path of length $\geq 5$ between $Y$-complete vertices, and none of its internal vertices are $Y$-complete, contrary to 13.6 . So $|X| \geq 2$, and similarly $|Y| \geq 2$. Hence there are at least two vertices $x \in X$ such that $X \backslash\{x\}$ is anticonnected, and from (2), $X \cap Y=\emptyset$, and there is a unique vertex $x \in X$ with nonneighbours in $Y$. By (2), there do not exist two vertices $x^{\prime} \in X \backslash\{x\}$ such that $X \backslash\left\{x^{\prime}\right\}$ is anticonnected; and therefore $X$ is an antipath with one end $x^{\prime}$. Because of the symmetry between $X, Y$, the same applies for $Y$, and this proves (3).

Choose $t^{\prime}$ with $1 \leq t^{\prime} \leq t$, minimum such that $p_{1}$ is nonadjacent to $y_{t^{\prime}}$. (This is possible since $p_{1}$ is not $Y$-complete.) So $x_{1}-\cdots-x_{s^{-}} y_{1^{-}} \cdots-y_{t^{\prime}}-p_{1}$ is an antipath. Define $W=\left(X \backslash\left\{x_{1}\right\}\right) \cup\left\{y_{1}, \ldots, y_{t^{\prime}-1}\right\}$.

(4) For every subpath $P^{\prime}$ of $P$, if the ends of $P^{\prime}$ are adjacent to $x_{1}$, then there are an even number of $W$-complete edges in $P^{\prime}$.

For suppose not; then we may choose $P^{\prime}$ such that no internal vertex of $P^{\prime}$ is adjacent to $x_{1}$. Let $P^{\prime}$ be $p_{h^{-}} \cdots-p_{k}$ say, where $1 \leq h<k \leq n$. Choose $i, j$ with $h \leq i \leq j \leq k$ such that $p_{i}, p_{j}$ are $W$-complete, with $i$ minimum and $j$ maximum. Since $p_{k}$ is not $X$-complete it follows that $p_{k}$ is not $W$-complete (because it is adjacent to $x_{1}$ ), and so $j<k$. Since there are an odd number of $W$-complete edges in $p_{h^{-}} \cdots-p_{k}$, it follows that $k \geq h+2$, and $x_{1}-p_{h^{-}} \cdots-p_{k}-x_{1}$ is a hole (so $k-h$ is even), containing an odd number of $W$-complete edges. By 2.3 it contains exactly one, and only two $W$-complete vertices; so $j=i+1$. The path $z$ - $x_{1}-p_{h^{-}} \cdots-p_{i}$ has both ends $W$-complete, and no internal vertex $W$-complete, and the $W$-complete vertex $p_{j}$ has no neighbour in its interior (since $j<k$ ); so it is even, by 2.2, and hence $i-h$ is even. Since $k-h$ is even, it follows that $p_{j^{-}} \cdots-p_{k^{-}} x_{1^{-}} z$ is an odd path; and again its ends are $W$-complete and its internal vertices are not. By 13.6 it has length 3 , so $k=j+1$; and by 2.2 , every $W$-complete vertex is adjacent to one of $p_{k}, x_{1}$. But no $W$-complete vertex in $P$ is adjacent to $x_{1}$ except $p_{1}$, since no other vertex of $P$ is $X$-complete. So every $W$-complete vertex in $P \backslash p_{1}$ is adjacent to $p_{k}$, and so must be one of $p_{k-1}, p_{k+1}$. In particular, since $i<k-1$ it follows that $i=1$, and so $j=2, k=3$, and the $W$-complete vertices in $P$ are $p_{1}, p_{2}$ and possibly $p_{4}$.

By 17.4 (with $X$ and $Y$ exchanged), $p_{2}$ is nonadjacent to $y_{t^{\prime}}$. Choose $d$ with $1 \leq d \leq n$ minimum such that $y_{t^{\prime}}$ is adjacent to $p_{d}$; then $d \geq 3$. Then the path $p_{1^{-}} \cdots-p_{d^{-}} y_{t^{\prime}} z$ has length $\geq 4$, and its ends are $W \cup\left\{x_{1}\right\}$-complete, and its internal vertices are not, so it is even by 13.6. Hence $d$ is odd, and the path $p_{1}-\cdots-p_{d^{-}} y_{t^{\prime}}$ is odd. None of its internal vertices are $X$-complete, and the $X$-complete vertex $z$ has no neighbour in its interior, and one end $p_{1}$ is $X$-complete, so the other end $y_{t^{\prime}}$ is not; and hence $t^{\prime}=1$, since all other vertices of $Y$ are $X$-complete. So $W=X \backslash\left\{x_{1}\right\}$. Let $V=X \backslash\left\{x_{s}\right\}$. 
Now the path $p_{1}-\cdots-p_{d^{-}} y_{1}$ is between $V$-complete vertices, and is odd and has length $>1$, and the $V$-complete vertex $z$ has no neighbour in its interior; so by 2.2 , there is a $V$-complete edge in its interior. Choose $c$ with $2 \leq c \leq d$ minimum such that $p_{c}$ is $V$-complete. Since $p_{2}$ is nonadjacent to $x_{1}$ it follows that $c \geq 3$. Since $p_{1}-\cdots-p_{c}$ is between $V$-complete vertices and its internal vertices are not $V$-complete and $z$ has no neighbour in it, it is even by 2.2, and so $c$ is odd. We already saw that $p_{1}, p_{2}$ and possibly $p_{4}$ are $W$-complete, and $c \geq 3$, so we may choose $b$ with $2 \leq b \leq c$ maximum such that $p_{b}$ is $W$-complete. Hence $b=2$ or 4 . The path $p_{b^{-}}-p_{c}$ is odd, and $p_{b}$ is $W$-complete, and $p_{c}$ is $V$-complete, and no other vertices of the path are either $W$ - or $V$-complete. If $c-b>1$ then $p_{b^{-}} \cdots-p_{c}, W, V$ is a counterexample to the theorem, contradicting the optimality of $X, Y, P$. So $c=b+1$. Then $z-p_{b^{-}} x_{1^{-}} \cdots-x_{s^{-}} p_{c^{-}} z$ is an antihole, so $s$ is odd. But then $p_{2^{-}} x_{1^{-}} \cdots-x_{s^{-}} y_{1}-p_{2}$ is an odd antihole, a contradiction. This proves (4).

Choose $h$ with $1 \leq h \leq n$ maximum such that $x_{1}$ is adjacent to $p_{h}$. Since $x_{1}-p_{h^{-}} \cdots-p_{n}$ is between $Y$-complete vertices (since $s \geq 2$ ) and none of its internal vertices are $Y$-complete, and the $Y$-complete vertex $z$ has no neighbour in its interior, this path either has length 1 or even length by 2.2. So either $h=n$ or $h$ is odd. From the optimality of $P, X, Y$, it follows that $P, W, Y$ is not a counterexample to the theorem, and so there are an odd number of $W$-complete edges in $P$. Since $x_{1}$ is adjacent to $p_{1}$, from (4) there are an even number of $W$-complete edges between $p_{1}$ and $p_{h}$, so there are an odd number in the path $p_{h^{-}} \cdots-p_{n}$, and in particular $h<n$, so $h$ is odd. Choose $i, j$ with $h \leq i \leq j \leq n$ such that $p_{i}, p_{j}$ are $W$-complete, with $i$ minimum and $j$ maximum. Hence $j>i$. Since $z-x_{1}-p_{h^{-}} \cdots-p_{i}$ is a path of length $\geq 2$ between $W$-complete vertices, and its internal vertices are not $W$-complete, and the $W$-complete vertex $p_{j}$ has no neighbour in its interior, it follows from 2.2 that $i-h$ is even.

(5) $h>1$.

For assume $h=1$; so $p_{1}$ is the only neighbour of $x_{1}$ in $P$. Let $S$ be the antipath

$$
x_{1}-\cdots-x_{s^{-}}-y_{1}-\cdots-y_{t}^{\prime}-p_{1} \text {. }
$$

Now $x_{1}-S-p_{1}-z$ is an antipath, of length $\geq 4$; all its internal vertices have neighbours in $P \backslash p_{1}$, and its ends do not. By 13.6 applied in $\bar{G}$, it follows that this antipath has even length and so $S$ has odd length. Its ends have no neighbours in $P \backslash\left\{p_{1}, p_{2}\right\}$, and $z$ is complete to its interior and also has no neighbours in $P \backslash\left\{p_{1}, p_{2}\right\}$; so by 2.2 applied in $\bar{G}$, some internal vertex of $S$ has no neighbour in $P \backslash\left\{p_{1}, p_{2}\right\}$. But they are all adjacent to $p_{j}$ or to $p_{n}$, so $j=2$. By 17.4, $p_{2}$ is nonadjacent to $y_{t^{\prime}}$, and also to $x_{1}$ since it is not $X$-complete. Therefore $p_{2}-x_{1^{-}} \cdots-x_{s^{-}} y_{1^{-}} \cdots-y_{t^{\prime}}-p_{2}$ is an antihole $D$ say. Choose $d$ with $1 \leq d \leq n$ minimum such that $y_{t^{\prime}}$ is adjacent to $p_{d}$; then $d \geq 3$, and so $x_{1}-p_{1^{-}} \cdots-p_{d^{-}} y_{t^{\prime}}-x_{1}$ is a hole of length $\geq 6$, with three vertices in common with $D$, namely $p_{2}, x_{1}, y_{t^{\prime}}$. From 15.7, $D$ has length 4 , and so $t^{\prime}=1$ and $s=2$. Since $W=\left\{x_{2}\right\}$ and $j=2$, it follows that the only edges between $x_{1}, x_{2}$ and $P$ are $x_{1} p_{1}, x_{2} p_{1}, x_{2} p_{2}$. But then the three paths $p_{1}-x_{1}, x_{2^{-}} z, p_{2^{-}} \cdots-p_{d^{-}} y_{1}$ form a long prism, a contradiction. This proves (5).

From (5), since $p_{h}$ is adjacent to $x_{1}$, it follows that $p_{h}$ is not complete to $X \backslash\left\{x_{1}\right\}$, and therefore $h<$ $i<j$. Choose $s^{\prime}$ with $1 \leq s^{\prime} \leq s$ minimum such that $p_{h}$ is nonadjacent to $x_{s^{\prime}}$. So $p_{j^{-}} x_{1^{-}} \cdots-x_{s^{\prime}}-p_{h^{-}} p_{j}$ is an antihole, and so $s^{\prime}$ is even. Hence $x_{1}-\cdots-x_{s^{\prime}}-p_{h^{-}} z$ is an odd antipath; all its internal vertices have neighbours in $\left\{p_{h+1}, \ldots, p_{n}\right\}$, and its ends do not, so by 13.6 it has length 3 , that is, $s^{\prime}=2$. The 
set $F=\left\{x_{2}, p_{h}, \ldots, p_{n}\right\}$ is connected; the only neighbour of $x_{1}$ in $F$ is $p_{h}$; the only neighbour of $z$ in $F$ is $x_{2}$. Since $x_{1}, z$ are $\left(X \backslash\left\{x_{1}, x_{2}\right\}\right) \cup Y$-complete, and $p_{h}, x_{2}$ are not (for $p_{h}$ is not $Y$-complete), it follows from 17.2 that there is a vertex in $\left(X \backslash\left\{x_{1}, x_{2}\right\}\right) \cup Y$ with no neighbour in $F$ except possibly $x_{2}$. But every vertex in $\left(X \backslash\left\{x_{1}, x_{2}\right\}\right) \cup Y$ is adjacent to either $p_{j}$ or to $p_{n}$, a contradiction. This proves 17.5 .

\section{Pseudowheels}

Let us say a pseudowheel in a graph $G$ is a triple $(X, Y, P)$, satisfying:

- $X, Y$ are disjoint nonempty anticonnected subsets of $V(G)$, complete to each other

- $P$ is a path $p_{1^{-}} \cdots-p_{n}$ of $G \backslash(X \cup Y)$, where $n \geq 5$

- $p_{1}, p_{n}$ are the only $X$-complete vertices of $P$

- $p_{1}$ is $Y$-complete, and so is at least one other vertex of $P$; and $p_{2}, p_{n}$ are not $Y$-complete.

A wheel $(C, Y)$ with a $Y$-segment $S$ of length one can be viewed as a pseudowheel, taking $X$ to consist of one of the vertices of $S$. We recommend that the reader think of a general pseudowheel as such an odd wheel, where a vertex of $S$ has "blown up" to become the anticonnected set $X$.

Our current goal is to prove an analogue of 16.3 for pseudowheels. Fortunately we don't need to generalize 16.3 completely, just the case when there is a segment of the wheel of length 1 , and one of its vertices has blown up. We did in fact try to generalize 16.3 completely, but were unable to do it and it gave us a lot of trouble; so eventually we found a way to make do with this special case.

We begin with an even more special case, a form of 15.3 when one vertex is replaced by an anticonnected set.

18.1 Let $G \in \mathcal{F}_{7}$, and let $X, Y$ be disjoint nonempty anticonnected subsets of $V(G)$, complete to each other. Let $p_{1}-p_{2}-p_{3}-p_{4}-p_{5}$ be a track in $G \backslash(X \cup Y)$, induced except possibly for the edge $p_{2} p_{5}$. Let $X$ be complete to $p_{1}, p_{5}$ and not to $p_{2}, p_{3}, p_{4}$. If $p_{1}, p_{3}, p_{4}$ are $Y$-complete then so is one of $p_{2}, p_{5}$.

Proof. Assume not. Then in $\bar{G},\left\{p_{1}, p_{3}, p_{5}\right\}$ is a triangle, and the connected set $F=X \cup Y \cup\left\{p_{2}, p_{4}\right\}$ catches it. In $\bar{G}$, the only neighbours of $p_{5}$ in $F$ are in $Y \cup\left\{p_{2}\right\}$, the only neighbours of $p_{3}$ in $F$ are in $X$, and the only neighbour of $p_{1}$ in $F$ is $p_{4}$. Hence no vertex of $F$ has two neighbours in the triangle, so by 17.1, $F$ contains a reflection of the triangle. So (back in $G$ ) there are vertices $b_{1} \in X$ and $b_{2} \in Y \cup\left\{p_{2}\right\}$ such that $b_{1}, b_{2}, p_{4}$ are pairwise nonadjacent, and $b_{1}$ is adjacent to $p_{1}, p_{5}$ and not $p_{3}$, and $b_{2}$ is adjacent to $p_{1}, p_{3}$ and not $p_{5}$. Since $p_{4}$ is $Y$-complete and $b_{2}, p_{4}$ are nonadjacent it follows that $b_{2} \notin Y$, and so $b_{2}=p_{2}$, and $p_{2}$ is not adjacent to $p_{5}$. Then $Y$ and the six vertices $p_{1}, \ldots, p_{5}, b_{1}$ form an odd wheel, a contradiction. This proves 18.1.

There is a reformulation of 13.7 that we sometimes need:

18.2 Let $G \in \mathcal{F}_{7}$, and let $X, Y$ be disjoint nonempty anticonnected subsets of $V(G)$, complete to each other. Let $P$ be a path in $G$ with even length $>0$, with vertices $p_{1}, \ldots, p_{n}$ in order, such that $p_{1}$ is $X$-complete, $p_{n}$ is not $X$-complete and $p_{n}$ is the unique $Y$-complete vertex of $P$. Suppose that there is a $Y$-complete vertex in $G$ nonadjacent to both $p_{n-1}, p_{n-2}$. Then either: 
- there is an odd number of $X$-complete edges in $P$, or

- $n=3$ and there is an odd antipath joining $p_{n-1}$ and $p_{n}$ with interior in $X$.

Proof. Choose an $X$-complete vertex $p_{i}$ in $P$ with $i$ maximum. Suppose first that $i$ is even. Then the path $p_{1}-\cdots-p_{i}$ is odd, and we may assume that an even number of its edges are $X$-complete. So it has length $>1$; by 2.3 , none of its internal vertices are $X$-complete; and by 13.6 it has length 3 (that is, $i=4$ ), and there is an odd antipath $Q$ joining $p_{2}, p_{3}$ with interior in $X$. Let $R$ be an antipath joining $p_{2}, p_{3}$ with interior in $Y$. Since $n \geq i=4$ and $n$ is odd, it follows that $n \geq 5$, and so one of $p_{2}-R-p_{3}-Q-p_{2}, p_{n}-p_{2}-R-p_{3}-p_{n}$ is an odd antihole, a contradiction.

Thus $i$ is odd. Hence the path $p_{i^{-}} \cdots-p_{n}$ is even, and by 13.7 it has length 2 , that is, $i=n-2$. Let $Q$ be the antipath between $p_{n-2}, p_{n-1}$ with interior in $Y$, and $R$ the antipath between $p_{n-1}, p_{n}$ with interior in $X$. By hypothesis there is a $Y$-complete vertex nonadjacent to $p_{n-1}, p_{n-2}$, and therefore $Q$ is even, so $R$ is odd by 13.7 . Hence $R$ cannot be completed to an antihole via $p_{n}-p_{1}-p_{n-1}$; and so $n=3$ and the theorem holds. This proves 18.2 .

We need the following extension of 2.3 .

18.3 Let $G \in \mathcal{F}_{7}$, and let $X, Y$ be disjoint nonempty anticonnected subsets of $V(G)$, complete to each other. Let $P$ be a path $p_{1}-\cdots-p_{n}$ of $G \backslash(X \cup Y)$, where $n \geq 5$, such that $p_{1}, p_{n}$ are the only $X$-complete vertices of $P$. Then $P$ has even length. Assume that at least two vertices of $P$ are $Y$ complete, and let $P^{\prime}$ be a maximal subpath of $P$ such that none of its internal vertices are $Y$-complete. Then the length of $P^{\prime}$ has the same parity as the number of ends of $P^{\prime}$ that belong to $\left\{p_{1}, p_{n}\right\}$ and are not $Y$-complete. Moreover, the number of $Y$-complete edges of $P$ has the same parity as the number of elements of $\left\{p_{1}, p_{n}\right\}$ that are $Y$-complete.

Proof. Since $P$ is a path of length $\geq 4$, and its ends are $X$-complete and its internal vertices are not, it follows that $P$ has even length, by 13.6. Let us say a line is a maximal subpath $P^{\prime}$ of $P$ such that no internal vertex of $P^{\prime}$ is $Y$-complete. Let $P^{\prime}$ be a line of length $\geq 2$, and assume first that both ends of $P^{\prime}$ are $Y$-complete. Suppose $P^{\prime}$ has odd length, and let its ends be $p_{i}, p_{j}$ where $i<j$. Then 13.6 implies that $j-i=3$, and there is an odd antipath $Q$ joining $p_{i+1}, p_{i+2}$ with interior in $Y$. Since $n \geq 5$, either $n>j$ or $1<i$, and from the symmetry between $p_{1}$ and $p_{n}$ we may assume the latter. Since $p_{i+1}, p_{i+2}$ are not $X$-complete, they are joined by an antipath $Q^{\prime}$ with interior in $X$. Since $Q \cup Q^{\prime}$ is an antihole it follows that $Q^{\prime}$ is odd. But then $p_{1}-p_{i+1}-Q^{\prime}-p_{i+2}-p_{1}$ is an odd antihole, a contradiction. So in this case $P^{\prime}$ has even length. We may therefore assume that an end of $P^{\prime}$ is not $Y$-complete, and from the maximality of $P^{\prime}$, any such end is either $p_{1}$ or $p_{n}$, and we may assume it is $p_{n}$ from the symmetry. The other end of $P^{\prime}$ is therefore not $p_{1}$ since at least two vertices of $P$ are $Y$-complete, and so it is $p_{i}$, where $i$ is maximum with $2 \leq i \leq n$ such that $p_{i}$ is $Y$-complete. Since $i>1$, no vertex of $P^{\prime}$ is $X$-complete except $p_{n}$. Suppose that $P^{\prime}$ is even; then we may apply 13.7. We deduce that $P^{\prime}$ has length 2 , and so $i=n-2$. Now the antipath joining $p_{n-2}, p_{n-1}$ with interior in $X$ is even since it can be completed to an antihole via $p_{n-1}-p_{1}-p_{n-2}$; and the antipath joining $p_{n-1}, p_{n}$ with interior in $Y$ is even since it can be completed to an antihole via $p_{n-} p_{h-} p_{n-1}$, where $p_{h}$ is some $Y$-complete vertex with $1 \leq h<i$. But this contradicts 13.7. Consequently $P^{\prime}$ is odd, as required.

We have shown therefore that a line has odd length if and only if either it has length 1 , or one of its ends is one of $p_{1}, p_{n}$ and is not $Y$-complete. It follows that the number of odd lines equals $y+z$, 
where $y$ is the number of $Y$-complete edges in $P$, and $z$ is the number of ends of $P$ that are not $Y$-complete. But since every edge of $P$ belongs to a unique line and $P$ has even length, it follows that the number of odd lines is even, and so $y, z$ have the same parity. This proves the final claim of the theorem, and so proves 18.3.

18.4 Let $(X, Y, P)$ be a pseudowheel in a graph $G \in \mathcal{F}_{7}$, where $P$ is $p_{1}-\cdots-p_{n}$. Then $P$ contains an odd number, at least 3 , of $Y$-complete edges, and $P$ has length $\geq 6$.

Proof. By 18.3, $P$ contains an odd number of $Y$-complete edges, since an odd number of ends of $P$ are $Y$-complete. Suppose it only contains one, say $p_{i} p_{i+1}$. Since $p_{2}, p_{n}$ are not $Y$-complete it follows that $3 \leq i \leq n-2$. So there is an antipath joining $p_{i}, p_{i+1}$ with interior in $X$, and by 15.4 applied to the path $P$, this antipath has length 2 , that is, there exists $x \in X$ nonadjacent to both $p_{i}, p_{i+1}$. Let $C$ be a hole containing $x, p_{i}, p_{i+1}$ and with $C \backslash x \subseteq P$. Then $(C, Y)$ is an odd wheel, since $C$ contains the $Y$-complete vertices $x, p_{i}, p_{i+1}$ and it also contains $p_{i-1}, p_{i+2}$ which are not $Y$-complete, contrary to $G \in \mathcal{F}_{7}$. So at least three edges of $P$ are $Y$-complete, and therefore $P$ has length $\geq 6$. This proves 18.4 .

A pseudowheel $(X, Y, P)$ in $G$ is optimal if

- there is no pseudowheel $\left(X^{\prime}, Y^{\prime}, P^{\prime}\right)$ in $G$ such that the number of $Y^{\prime}$-complete vertices in $P^{\prime}$ is less than the number of $Y$-complete vertices in $P$, and

- there is no pseudowheel $\left(X, Y^{\prime}, P\right)$ in $G$ such that $Y \subset Y^{\prime}$.

18.5 Let $G \in \mathcal{F}_{7}$, and let $(X, Y, P)$ be an optimal pseudowheel in $G$, where $P$ is $p_{1}-\cdots-p_{n}$. Let $v \in V(G) \backslash(X \cup Y \cup V(P))$, not $Y$-complete. Then there is a subpath $P^{\prime}$ of $P$ such that

- $V\left(P^{\prime}\right)$ contains all the neighbours of $v$ in $P$,

- there is no $Y$-complete vertex in the interior of $P^{\prime}$, and

- if $v$ is $X$-complete, then either $V\left(P^{\prime}\right)=\left\{p_{1}\right\}$, or $p_{n} \in V\left(P^{\prime}\right)$.

Proof. Choose $h, k$ with $1 \leq h \leq k \leq n$ such that $v$ is adjacent to $p_{h}, p_{k}$, with $h$ minimum and $k$ maximum. (If this is impossible then the theorem holds.) Choose $i, j$ with $2 \leq i \leq j \leq n$ such that $p_{i}, p_{j}$ are $Y$-complete, with $i$ minimum and $j$ maximum. By 18.3 it follows that $i$ is odd and $j$ is even, and $j-i \geq 3$ by 18.4 , since all $Y$-complete edges in $P$ lie in the path $p_{i^{-}} \cdots-p_{j}$.

(1) If $v$ is both adjacent to $p_{1}$ and $X$-complete then the theorem holds.

For from the optimality of $(X, Y, P)$ it follows that $(X, Y \cup\{v\}, P)$ is not a pseudowheel, and so $p_{1}$ is the only $Y \cup\{v\}$-complete vertex in $P$. By 2.11 (with $X, Y$ replaced by $Y \cup\{v\}, X$ ) we deduce that either there exists $y \in Y \cup\{v\}$ nonadjacent to all $p_{2}, \ldots, p_{n}$, or there exist nonadjacent $y_{1}, y_{2} \in Y \cup\{v\}$ such that $y_{1}-p_{2}-\cdots-p_{n}-y_{2}$ is a path. But $p_{i}$ is $Y$-complete and $3 \leq i \leq n-1$, so the second statement does not hold; and the first holds only if $y=v$. This proves (1).

(2) We may assume that there is a path $Q$ from $v$ to some vertex $q$, such that $q$ is the only $Y$ complete vertex in $Q$, and $V(Q \backslash v) \subseteq\left\{p_{i+1}, \ldots, p_{j-1}\right\}$. 
For by 18.3 and the fact that there is a $Y$-complete edge in $P$, it follows that there is a $Y$-complete vertex in $\left\{p_{i+1}, \ldots, p_{j-1}\right\}$. If $v$ has a neighbour in this set then the claim holds, so suppose it does not. We may assume $v$ has a neighbour in $\left\{p_{1}, \ldots, p_{i}\right\}$, for otherwise the theorem holds. Suppose it also has a neighbour in $\left\{p_{j}, \ldots, p_{n}\right\}$. Then there is a hole $C$ containing $v$, with $C \backslash v \subseteq P$, such that $p_{i^{-}} \cdots-p_{j}$ is a path of $C$. Since all $Y$-complete edges in $P$ belong to this path, and there are an odd number of them, it follows that there is an odd number $(\geq 3)$ of $Y$-complete edges in $C$, contrary to 2.3. So $v$ has no neighbours in $\left\{p_{j}, \ldots, p_{n}\right\}$, and hence $k \leq i$. We may therefore assume that $v$ is $X$-complete, so $k>1$ by (1). The path $v-p_{k^{-}} \cdots-p_{n}$ has length $\geq 4$, and its ends are $X$-complete, and its internal vertices are not, so by 13.6 it has even length, and therefore the path $v-p_{k^{-}} \cdots-p_{i}$ is even. But $v$ is the only $X$-complete vertex in $v-p_{k^{-}} \cdots-p_{i}$, and $p_{i}$ is its only $Y$-complete vertex (since $k>1$ ), so by 13.7 , this path has length 2 , and so $k=i-1$. There is no odd antipath joining $v, p_{k}$ with interior in $Y$, since the $Y$-complete vertex $p_{j}$ is nonadjacent to $v, p_{k}$; and there is no odd antipath joining $p_{k}, p_{i}$ with interior in $X$, since the $X$-complete vertex $p_{n}$ is nonadjacent to $p_{k}, p_{i}$, contrary to 13.7 . This proves (2).

\section{(3) If $v$ is $X$-complete then the theorem holds.}

For then we may assume that $v$ is nonadjacent to $p_{1}$ by (1). If $h$ is odd then $p_{1^{-}} \cdots-p_{h^{-}} v$ is an odd path with ends $X$-complete and its internal vertices not, so it has length 3 by 13.6 ; but the $X$-complete vertex $p_{n}$ has no neighbour in its interior ( $\operatorname{since} n \geq 5$ ), contrary to 2.2. So $h$ is even. Suppose that one of $p_{2}, \ldots, p_{h}$ is $Y$-complete. Then $h \neq 2$ since $p_{2}$ is not $Y$-complete, so $h \geq 4$, and $h<j$ by $(2)$. Hence $\left(X, Y, p_{1}-\cdots-p_{h^{-}} v\right)$ is a pseudowheel, not containing $p_{j}$, contrary to the optimality of $(P, X, Y)$. So there are no $Y$-complete vertices in $\left\{p_{2}, \ldots, p_{h}\right\}$, and so $i>h$. Let $Q, q$ be as in (2). Since the $X \cup Y$-complete vertex $p_{1}$ has no neighbours in $Q$, the pairs $(V(Q), X),(V(Q), Y)$ are balanced by 2.6 ; so by $2.9, Q$ has odd length. Hence the path $p_{1^{-}} \cdots-p_{h^{-}}-Q-q$ has odd length, and its ends are $Y$-complete, and its internal vertices are not. By 13.6 it has length 3 ; so $h=2$ and $v$ is adjacent to $q$. Also every $Y$-complete vertex in $P$ is adjacent to one of $v, p_{2}$, by 2.2 , so they are all adjacent to $v$ except $p_{1}$ and possibly $p_{3}$. Suppose $p_{i}$ is adjacent to $v$, and is therefore $Y \cup\{v\}$-complete. The path $p_{1}-\cdots-p_{i}$ has even length; the only $X$-complete vertex in it is $p_{1}$; and the only $Y \cup\{v\}$-complete vertex in it is $p_{i}$. By 13.7 it has length 2. But $p_{n}$ is an $X$-complete vertex nonadjacent to both $p_{2}, p_{3}$, and $p_{j}$ is a $Y \cup\{v\}$-complete vertex nonadjacent to both $p_{1}, p_{2}$, since $j-i \geq 3$ and therefore $p_{j}$ is is necessarily adjacent to $v$ as we already saw. Hence both pairs $\left(\left\{p_{1}, p_{2}\right\}, Y \cup\{v\}\right)$ and $\left(\left\{p_{2}, p_{3}\right\}, X\right)$ are balanced by 2.6, contrary to 13.7. This proves that $p_{i}$ is not adjacent to $v$, and therefore $i=3$. Choose $h^{\prime}>i$ minimum such that $v$ is adjacent to $p_{h^{\prime}}$. From the hole $v-p_{2^{-}} \cdots-p_{h^{\prime}} v$ it follows that $h^{\prime}$ is even. From 18.2 applied to the even path $p_{3^{-}} \cdots-p_{h^{\prime}} v$, and using the fact that the $X \cup Y$-complete vertex $p_{1}$ has no neighbour in this path, we deduce that there is a $Y$-complete edge in $p_{3}-\cdots-p_{h^{\prime}}-v$. Since $v$ is adjacent to every $Y$-complete vertex in $P$ except $p_{1}, p_{3}$, it follows that the only such edge is $p_{3} p_{4}$, and therefore $h^{\prime}=4$. But then the track $p_{1}-\cdots-p_{4}-v$ violates 18.1. This proves (3).

Henceforth we may therefore assume that $v$ is not $X$-complete. If $k \leq h+1$ then the theorem holds, so we assume $k \geq h+2$.

(4) If $v$ is not adjacent to $p_{1}$ then the theorem holds. 
For let $P^{\prime}$ be the path $p_{1^{-}} \cdots-p_{h^{-}} v-p_{k^{-}} \cdots-p_{n}$. Suppose that any of $p_{2}, \ldots, p_{h}, p_{k}, \ldots, p_{n}$ is $Y$-complete. Then $P^{\prime}$ has length $\geq 4$, since $h>1$ and $p_{2}, p_{n}$ are not $Y$-complete, and so $\left(X, Y, P^{\prime}\right)$ is a pseudowheel. By the optimality of $(X, Y, P)$ it follows that there are no $Y$-complete vertices among $\left\{p_{h+1}, \ldots, p_{k-1}\right\}$; but then the claim holds. So we may assume that none of $p_{2}, \ldots, p_{h}, p_{k}, \ldots, p_{n}$ is $Y$-complete, and therefore $h<i \leq j<k$, and since $j-i \geq 3$ it follows that $k-h \geq 5$. Let $Q, q$ be as in (2). Then $q-Q-v-p_{k^{-}} \cdots-p_{n}$ is a path, $R$ say; the only $Y$-complete vertex in $R$ is $q$; the only $X$-complete vertex in $R$ is $p_{n}$; and the $X \cup Y$-complete vertex $p_{1}$ has no neighbour in its interior. By $2.9, R$ is odd. Therefore the paths $p_{1^{-}} \cdots-p_{h}-v-Q-q$ and $p_{1^{-}} \cdots-p_{h}-v-p_{k^{-}} \cdots-p_{n}$ have lengths of opposite parity. For the first path, its ends are $Y$-complete and its internal vertices are not. For the second, its ends are $X$-complete and its internal vertices are not. So by 13.6, one of them has length 3 , and so $h=2$, and there is an odd antipath joining $v, p_{2}$ with interior in one of $X, Y$. Since $v, p_{2}$ are joined by an antipath with interior in $X$ and by another with interior in $Y$, and all such pairs of antipaths have the same parity (since their union is an antihole), it follows that $v, p_{2}$ are joined by an odd antipath with interior in each of $X, Y$. Hence every $X$-complete vertex is adjacent to one of $v, p_{2}$, and so is every $Y$-complete vertex. In particular $k=n$, and $v$ is adjacent to every $Y$-complete vertex in $P$ except $p_{1}$ and possibly $p_{3}$. But then $R$ has length 2 , contradicting that it has odd length. This proves (4).

Henceforth then we assume that $v$ is adjacent to $p_{1}$ and not $X$-complete.

$p_{n-1}$ is not $Y \cup\{v\}$-complete.

For suppose it is. Since $n \geq 7$, it follows from 13.6 applied to $P \backslash p_{n}$ and $Y \cup\{v\}$ that there is a $Y \cup\{v\}$-complete vertex in $\left\{p_{2}, \ldots, p_{n-2}\right\}$; choose such a vertex, $p_{j^{\prime}}$ say, with $j^{\prime}$ maximum. Now $j=n-1$. If $j^{\prime}<j-1$ then $j-j^{\prime}$ is even from 2.2 applied to $p_{j^{\prime}} \cdots-p_{j}$, since $p_{1}$ is $Y \cup\{v\}$-complete and has no neighbours in the interior of $p_{j^{\prime}} \cdots-p_{j}$; but then the odd path $p_{j^{\prime}} \cdots-p_{n}$ contains no $Y \cup\{v\}$-complete edges, and $p_{1}$ is $X$-complete, $Y \cup\{v\}$-complete and has no neighbours in the path $p_{j^{\prime}} \cdots-p_{n}$, contrary to 17.5. So $j^{\prime}=j-1$. Let $F=X \cup Y \cup\left\{v, p_{n-1}\right\}$. Then $F$ is anticonnected, and each of $p_{1}, p_{n-2}, p_{n}$ has a nonneighbour in $F$; the only nonneighbour of $p_{1}$ in $F$ is $p_{n-1}$; all nonneighbours of $p_{n-2}$ in $F$ belong to $X$; and all nonneighbours of $p_{n}$ in $F$ belong to $Y \cup\{v\}$. So in $\bar{G}$, the connected set $F$ catches the triangle $\left\{p_{1}, p_{n-2}, p_{n}\right\}$, and by 17.1 it contains a reflection of the triangle, which is impossible since $p_{n-1}$ is complete (in $G$ ) to $Y \cup\{v\}$. This proves (5).

(6) $v$ is not adjacent to $p_{n}$.

For suppose it is. By 18.4 there are at least three $Y$-complete edges in $P$, and so there is a $Y$ complete vertex $p_{a}$ in $P$ with $a \geq 3$, even and different from $p_{n-1}$. Thus $j-a$ is even, and so by 2.3 there is an even number of $Y$-complete edges in the even path $p_{a^{-}} \cdots-p_{j}$, and hence in the odd path $p_{a^{-}} \cdots-p_{n}$. But $p_{a}$ is $Y$-complete, and $p_{n}$ is the unique $X \cup\{v\}$-complete vertex in this path, contrary to 17.5 . This proves (6).

(7) There is no neighbour $p_{m}$ of $v$ in $P$ with $1 \leq m \leq n$ such that $v, p_{m}$ are joined by an odd antipath with interior in $Y$. 
For suppose such a neighbour exists. So $1<m<n$ by (6), and there is an antipath joining $v, p_{m}$ with interior in $X$, which therefore is also odd, since its union with the antipath through $Y$ is an antihole. Since it cannot be completed to an odd antihole via $p_{m}-p_{n}-v$, it follows that $m=n-1$, and in particular $m$ is even. Since $j$ is even, either $p_{j}=p_{m}$ or $p_{j}$ is nonadjacent to $p_{m}$; and in either case it follows that $p_{j}$ is adjacent to $v$, since every $Y$-complete vertex is adjacent to one of $v, p_{m}$. By (5) $n-j \geq 3$ and odd, and the path $p_{j^{-}} \cdots-p_{n}$ (with anticonnected sets $X$ and $Y \cup\{v\}$ ) violates 17.5. This proves $(7)$.

Suppose that $j \geq k$, and let $P^{\prime}$ be the path $p_{1}-v-p_{k^{-}} \cdots-p_{n}$. Then $P^{\prime}$ has length $\geq 4$, since $p_{n-1}$ is not $Y \cup\{v\}$-complete, and so $\left(X, Y, P^{\prime}\right)$ is a pseudowheel; and by the optimality of $(X, Y, P)$ it follows that there are no $Y$-complete vertices in $p_{2^{-}} \cdots-p_{k-1}$, contrary to (2). So $j<k$. Let $Q, q$ be as in (2), and assume first that $Q$ is even. Then the path $p_{1}-v-Q-q$ has odd length; its ends are $Y$-complete, and its internal vertices are not, so by 13.6 it has length 3 , and its internal vertices are joined by an odd antipath with interior in $Y$, contrary to (7). So $Q$ is odd.

Next assume that $k$ is even. Then the path $p_{1}-v-p_{k^{-}} \cdots-p_{n}$ is odd, and its ends are $X$-complete, and its internal vertices are not, so by 13.6 it has length 3 , and $k=n-1$, and its internal vertices $v, p_{n-1}$ are joined by an odd antipath with interior in $X$. Since $p_{n-1}$ is not $Y \cup\{v\}$-complete, they are also joined by an odd antipath with interior in $Y$, contrary to (7). This proves that $k$ is odd. Hence the path $q-Q-v-p_{k^{-}} \cdots-p_{n}$ is even, and by (6) it has length $>2$ contrary to 13.7. This proves 18.5 .

18.6 Let $G \in \mathcal{F}_{7}$, and let $(X, Y, P)$ be an optimal pseudowheel in $G$, where $P$ is $p_{1}-\cdots-p_{n}$. Let $F \subseteq V(G) \backslash(X \cup Y \cup V(P))$ be connected, such that no vertex in $F$ is $Y$-complete. Then there is a subpath $P^{\prime}$ of $P$ such that

- $V\left(P^{\prime}\right)$ contains all the attachments of $F$ in $P$,

- there is no $Y$-complete vertex in the interior of $P^{\prime}$, and

- if some vertex of $F$ is $X$-complete then either $V\left(P^{\prime}\right)=\left\{p_{1}\right\}$ or $p_{n} \in V\left(P^{\prime}\right)$.

Proof. Suppose the theorem is false, and choose a minimal counterexample $F$. From $18.5|F| \geq 2$.

(1) Some vertex in $F$ is $X$-complete.

For suppose not. Since $F$ is a counterexample, it has attachments $p_{a}, p_{c}$ such that there is a $Y$-complete vertex $p_{b}$ with $a<b<c$. From the minimality of $F, F$ is the interior of a path $p_{a}-f_{1}-\cdots-f_{k}-p_{c}$. Let $W_{1}$ be the set of attachments of $F \backslash\left\{f_{k}\right\}$ in $P$, and $W_{2}$ the set of attachments of $F \backslash\left\{f_{1}\right\}$ in $P$. From the minimality of $F$, for $i=1,2$ there is a subpath $p_{a_{i}} \cdots-p_{b_{i}}$ of $P$ ( $=P_{i}$ say), such that no internal vertex of $P_{i}$ is $Y$-complete, and $W_{i} \subseteq V\left(P_{i}\right)$. Choose $P_{1}, P_{2}$ minimal; then $p_{a_{1}}$ is a neighbour of some member of $F \backslash\left\{f_{k}\right\}$, and therefore of $f_{1}$ from the minimality of $F$, and similarly $p_{b_{2}}$ is a neighbour of $f_{k}$, and $p_{1}-\cdots-p_{a_{1}}-f_{1^{-}} \cdots-f_{k}-p_{b_{2}}-\cdots-p_{n}$ is a path $P^{\prime}$ say. Suppose that there is a $Y$-complete vertex in $P^{\prime}$ different from $p_{1}$. Then $P^{\prime}$ has length $\geq 4$, and $\left(X, Y, P^{\prime}\right)$ is a pseudowheel, contrary to the optimality of $(X, Y, P)$. So there are no $Y$-complete vertices in $P^{\prime}$ different from $p_{1}$. But also there are none in $\left\{p_{a_{1}+1}, \ldots, p_{b_{1}-1}\right\}$ and none in $\left\{p_{a_{2}+1}, \ldots, p_{b_{2}-1}\right\}$, so all the $Y$-complete 
vertices of $P$ belong to $\left\{p_{b_{1}}, \ldots, p_{a_{2}}\right\}$, except for $p_{1}$. By 18.4 there are an odd number, at least 3 , of $Y$-complete edges in this path. From the minimality of $F, f_{1^{-}} \cdots-f_{k^{-}}-p_{a_{2}}-p_{a_{2}-1^{-}} \cdots-p_{b_{1}}-f_{1}$ is a hole, which therefore also contains an odd number $\geq 3$ of $Y$-complete edges. But this contradicts 2.3. This proves (1).

(2) There do not exist $a, b$ with $1<a<b \leq n$ such that $p_{a}$ is an attachment of $F$ and $p_{b}$ is $Y$-complete.

For suppose that such $a, b$ exist. From (1), there is an $X$-complete vertex in $F$; and from the minimality of $F$, there is a path $p_{a^{-}} f_{1^{-}} \cdots-f_{k}$ such that $F=\left\{f_{1}, \ldots, f_{k}\right\}$ and $f_{k}$ is the unique $X$-complete vertex in $F$. Let $W_{1}$ be the set of attachments of $F \backslash\left\{f_{k}\right\}$ in $P$, and $W_{2}$ the set of attachments of $F \backslash\left\{f_{1}\right\}$ in $P$. From the minimality of $F$, for $i=1,2$ there is a subpath $p_{a_{i}-\cdots-p_{b_{i}} \text { of }}$ $P\left(=P_{i}\right.$ say), such that no internal vertex of $P_{i}$ is $Y$-complete, and $W_{i} \subseteq V\left(P_{i}\right)$, and either $b_{2}=n$ or $a_{2}=b_{2}=1$.

First assume that $b_{2}=n$. Choose $P_{1}, P_{2}$ minimal; then $p_{a_{1}}$ is a neighbour of $f_{1}$, and $p_{1}-\cdots-p_{a_{1}}-f_{1^{-}} \cdots-f_{k}$ is a path $P^{\prime}$ say. Suppose that there is a $Y$-complete vertex in $P^{\prime}$ different from $p_{1}$. Then $P^{\prime}$ has length $\geq 4$, and $\left(X, Y, P^{\prime}\right)$ is a pseudowheel, contrary to the optimality of $(X, Y, P)$. So there are no $Y$-complete vertices in $P^{\prime}$. But also there are none in $\left\{p_{a_{1}+1}, \ldots, p_{b_{1}-1}\right\}$ and none in $\left\{p_{a_{2}+1}, \ldots, p_{b_{2}-1}\right\}$, so all the $Y$-complete vertices of $P$ belong to $\left\{p_{b_{1}}, \ldots, p_{a_{2}}\right\}$, except for $p_{1}$. By 18.4 there are an odd number, at least 3 , of $Y$-complete edges in this path. From the minimality of $F, f_{1}-\cdots-f_{k}-p_{a_{2}}-p_{a_{2}-1}-\cdots-p_{b_{1}}-f_{1}$ is a hole, which therefore also contains an odd number $\geq 3$ of $Y$-complete edges. But this contradicts 2.3.

So we may assume that $a_{2}=b_{2}=1$, and that $p_{1} \in W_{2}$, and therefore $b_{1}>1$. From the minimality of $F$ there are no edges between $F \backslash\left\{f_{1}\right\}$ and $V\left(P \backslash p_{1}\right)$. Choose $P_{1}$ minimal. So $p_{b_{1}}$ is adjacent to $f_{1}$, and either $a_{1}=1$ or $p_{a_{1}}$ is adjacent to $f_{1}$. Suppose first that an odd number of edges of the path $p_{1}-\cdots-p_{a_{1}}$ are $Y$-complete. Hence $p_{1}$ has no neighbours in $F \backslash\left\{f_{k}\right\}$, and so $f_{1}-\cdots-f_{k^{-}} p_{1^{-}} \cdots-p_{a_{1}}-f_{1}$ is a hole. It contains an odd number of $Y$-complete edges, and at least three $Y$-complete vertices, because $p_{1}$ is $Y$-complete and $p_{2}$ is not, a contradiction to 2.3. So there are an even number of $Y$-complete edges in the path $p_{1}-\cdots-p_{a_{1}}$, and therefore an odd number in $p_{b_{1}}-\cdots-p_{n}$, since there are an odd number in $P$, and none in $P_{1}$. Therefore there are an odd number in the path $f_{k^{-}} \cdots-f_{1}-p_{b_{1}}-\cdots-p_{n}\left(=R\right.$ say). But an edge of $p_{b_{1}}-\cdots-p_{n}$ is $Y$-complete and $p_{n}$ is not, so $b_{2} \leq n-2$; and since $k \geq 2$, it follows that $R$ has length $\geq 4$. Also, at least two vertices of $R$ are $Y$-complete, and its ends are not $Y$-complete, and its ends are its only $X$-complete vertices. This contradicts 18.3 , So there is no such $F$. This proves (2).

Choose $b$ with $1 \leq b \leq n$ maximum such that $p_{b}$ is $Y$-complete. By (2), none of $p_{2}, \ldots, p_{b-1}$ are attachments of $F$, and since $F$ is a counterexample, it follows that $p_{1}$ is an attachment of $F$ and also there exists $c$ with $b \leq c \leq n$ such that $p_{c}$ is an attachment of $F$. Choose $c$ with $c$ minimum, and let $Q$ be a path between $p_{1}, p_{c}$ with interior in $F$. Then $p_{1^{-}} \cdots-p_{c^{-}} Q-p_{1}$ is a hole, and the $Y$-complete edges in it are precisely the $Y$-complete edges in $P$. But there are an odd number of such edges and at least 3 , by 18.4, contrary to 2.3. Thus there is no such $F$. This proves 18.6.

Now we come to the main result of this section, 1.8.8, which we restate, the following.

18.7 Let $G \in \mathcal{F}_{7}$. If it contains a pseudowheel then it admits a balanced skew partition. In particular, every recalcitrant graph belongs to $\mathcal{F}_{8}$. 
Proof. Suppose $G$ contains a pseudowheel; then it contains an optimal pseudowheel, say $(X, Y, P)$, where $P$ is $p_{1}-\cdots-p_{n}$. Let $Z$ be the set of all $Y$-complete vertices in $G$. So $Y, Z$ are disjoint, nonempty, and complete to each other, and $|Z| \geq 2$. Let $F_{0}=V(G) \backslash(Y \cup Z)$. By 15.2, we may assume that $F_{0}$ is connected and every vertex in $Z$ has a neighbour in $F_{0}$, for otherwise the theorem holds. Choose $i>1$ such that $p_{i} p_{i+1}$ is $Y$-complete, and let $A, B$ be the two components of $V\left(P \backslash p_{i}\right)$. Since $p_{1}, p_{i+1}$ both have neighbours in $F_{0}$, it follows that $F_{0}$ contains a minimal connected set $F$ such that there are vertices in $A$ and in $B$ with neighbours in $F$. From the minimality of $F$ it is disjoint from $V(P)$; and disjoint from $X \cup Y$ since $X \subseteq Z$, contrary to 18.6. This proves 18.7.

\section{Wheel systems}

Henceforth, therefore, we can exclude pseudowheels, and so our graph $G$ belongs to $\mathcal{F}_{8}$. Please note that $G$ might still contain wheels; not every wheel can be converted to a pseudowheel. Our next goal is to show that if there is a wheel in a member of $\mathcal{F}_{8}$ then the graph admits a balanced skew partition, and in particular that there is no wheel in a recalcitrant graph. Assuming there is no balanced skew partition, the strategy is to show that there is no anticonnected set which is maximal such that there is a wheel of which it is a hub. In other words, we want to show that given any wheel, there is a second wheel whose hub is a proper superset of the hub of the first wheel. The proof of this is quite complex, and we begin with an overview before we launch into the details. But before the overview we need some definitions.

Let $G$ be a graph. A frame in $G$ is a pair $\left(z, A_{0}\right)$, where $z \in V(G)$, and $A_{0}$ is a nonnull connected subset of $V(G) \backslash\{z\}$, containing no neighbours of $z$. For the moment, fix a frame $\left(z, A_{0}\right)$. With respect to the given frame, a wheel system in $G$ of height $t \geq 1$ is a sequence $x_{0}, \ldots, x_{t}$ of distinct vertices of $G \backslash\left(A_{0} \cup\{z\}\right)$, satisfying the following conditions:

1. $A_{0}$ contains neighbours of $x_{0}$ and of $x_{1}$, and no vertex in $A_{0}$ is $\left\{x_{0}, x_{1}\right\}$-complete.

2. For $2 \leq i \leq t$, there is a connected subset of $V(G)$ including $A_{0}$, containing a neighbour of $x_{i}$, containing no neighbour of $z$, and containing no $\left\{x_{0}, \ldots, x_{i-1}\right\}$-complete vertex.

3. For $1 \leq i \leq t, x_{i}$ is not $\left\{x_{0}, \ldots, x_{i-1}\right\}$-complete.

4. $z$ is adjacent to all of $x_{0}, \ldots, x_{t}$.

Note that this definition is symmetric between $x_{0}, x_{1}$, so $x_{1}, x_{0}, x_{2}, \ldots, x_{t}$ is another wheel system.

A wheel system is defined with respect to a given frame, but it is convenient usually to leave the dependence on the frame implicit. Until 23.3 we shall always be working with a fixed frame, and all wheel systems are with respect to that frame.

Let $x_{0}, \ldots, x_{t}$ be a wheel system of height $t$. For $1 \leq i \leq t$ we define $X_{i}=\left\{x_{0}, \ldots, x_{i}\right\}$, and we define $A_{i}$ to be the maximal connected subset of $V(G)$ that includes $A_{0}$, contains no neighbour of $z$, and contains no $X_{i}$-complete vertex. So for each $i, A_{i-1} \subseteq A_{i}$. Note that condition 2 above just says that $x_{i}$ has a neighbour in $A_{i-1}$.

Let $x_{0}, \ldots, x_{t}$ be a wheel system, and let $Y$ be a nonempty anticonnected subset of $V(G) \backslash\left(A_{0} \cup\right.$ $\{z\})$. We say $Y$ is a $h u b$ for the wheel system if $z, x_{0}, \ldots, x_{t-1}$ are all $Y$-complete and $x_{t}$ is not.

Now we can begin the overview. Suppose there is a wheel system with hub $Y$. We would like to infer that there is a wheel with hub $Y$. This is not in general true, but our main theorem about wheel systems, the following, asserts that this is true under some mild extra hypotheses: 
19.1 Let $G \in \mathcal{F}_{8}$, let $\left(z, A_{0}\right)$ be a frame, and let $x_{0}, \ldots, x_{t+1}$ be a wheel system with hub $Y$, and with $t \geq 1$. Define $A_{i}, X_{i}$ as usual, and assume that at most one member of $Y$ has no neighbour in $A_{1}$. Suppose that for all $r$ with $1 \leq r \leq t$, if $x_{0}, x_{1}, \ldots, x_{r}, x_{t+1}$ is a wheel system, then every member of $Y$ has a neighbour in $A_{r} \cup\left\{x_{t+1}\right\}$. Then there is a wheel with hub $Y$.

The proof of this is lengthy, but here is the idea. Choose $r$ with $1 \leq r \leq t$, minimum such that $x_{t+1}$ has a neighbour in $A_{r}$ and a nonneighbour in $X_{r}$. By hypothesis, every member of $Y$ has a neighbour in $A_{r} \cup\left\{x_{t+1}\right\}$. From the minimality of $r$, either

- $r=1$, or

- $r>1$ and $x_{t+1}$ has a neighbour in $A_{r-1}$, and $x_{t+1}$ is $X_{r-1}$-complete, or

- $r>1$ and $x_{t+1}$ has no neighbour in $A_{r-1}$.

We handle these three cases separately; they are the results 19.2, 20.1, and 21.2 respectively. In the second case, we call the wheel system $x_{0}, x_{1}, \ldots, x_{r}, x_{t+1}$ a " $Y$-diamond", and prove the claim by induction on its height; and in this case, it turns out that the hypothesis that every member of $Y$ has a neighbour in $A_{r} \cup\left\{x_{t+1}\right\}$ is redundant (and indeed, so is the hypothesis that $z$ is $Y$-complete), and there is an advantage to relaxing these hypotheses, to strengthen the inductive hypothesis. The proof of 19.1 is completed in section 21.

Now let us sketch how 19.1 will be applied. The first application is to prove that no recalcitrant graph contains a wheel. For suppose that $(C, Y)$ is a wheel, with $Y$ maximal. Since it is not an odd wheel, there are three consecutive $Y$-complete vertices $x_{0}, z, x_{1}$ of $C$. Let $A_{0}=V(C) \backslash\left\{x_{0}, z, x_{1}\right\}$; then $\left(z, A_{0}\right)$ is a frame, and $x_{0}, x_{1}$ is a wheel system with respect to it. Since $G$ admits no balanced skew partition, there is a path $T$ from $z$ to $A_{0}$ so that no internal vertex of $T$ belongs to $Y$ or is $Y$-complete. Let $y$ be the neighbour of $z$ in $T$. If we choose the rim $C$ carefully, then because $G$ contains no pseudowheels, it can be shown (in the proof of 23.2) that $y$ is adjacent to $x_{0}, x_{1}$. Enlarge $x_{0}, x_{1}$ to a wheel system $x_{0}, \ldots, x_{t}$ such that $x_{0}, \ldots, x_{t}$ are all $Y \cup\{y\}$-complete, with $t$ maximum. Since we may assume that $G$ admits no balanced skew partition, there is a path $P$ from $z$ to $A_{0}$ so that no internal vertex of $T$ is in $X_{t}$ or is $X_{t}$-complete. Let $x_{t+1}$ be the neighbour of $z$ in $P$; then $x_{0}, \ldots, x_{t+1}$ is a wheel system, so from the maximality of $t, x_{t+1}$ has a nonneighbour in $Y \cup\{y\}$. Hence $Y \cup\{y\}$ is a hub for the wheel system $x_{0}, \ldots, x_{t+1}$. From the maximality of $Y$, there is no wheel with hub $Y \cup\{y\}$, and since every member of $Y$ has a neighbour in $A_{0}$, we deduce from 19.1 that there exists $r$ with $1 \leq r \leq t$, such that $x_{0}, x_{1}, \ldots, x_{r}, x_{t+1}$ is a wheel system, and $y$ has no neighbour in $A_{r} \cup\left\{x_{t+1}\right\}$. In particular, $y$ has only three neighbours in $C$. On the other hand, recall that $y$ was the second vertex of the path $T$ between $z$ and $A_{0}$. We deduce that the other neighbour of $y$ in $T$ does not belong to $A_{r} \cup\left\{x_{t+1}\right\}$, and therefore there are vertices of $T \backslash\{y, z\}$ that are $X_{r}$-complete. Since $G$ contains no pseudowheels, this turns out to be impossible, as we show in the proof of 22.4 .

There is another application of 19.1, to prove that in a recalcitrant graph, if $C$ is a hole of length at least 6 then no vertex has three consecutive neighbours in $C$. But this application (in the proof of 23.3) is much less convoluted, since at that stage we know there are no wheels, and we do not sketch it here.

The result of this section is the following. (Incidentally, we will not need the hypothesis that there is no pseudowheel in $G$ for several more sections. What we are proving here is true also for graphs in $\mathcal{F}_{7}$, and we formulate it that way, although we only need it for graphs in $\mathcal{F}_{8}$.) 
19.2 Let $G \in \mathcal{F}_{7}$, and let $\left(z, A_{0}\right)$ be a frame. Let $x_{0}, x_{1}, x_{2}$ be a wheel system with respect to this frame, and define $A_{1}$ as usual. Let $Y \subseteq V(G) \backslash\left\{z, x_{0}, x_{1}, x_{2}\right\}$ be anticonnected, such that

- $x_{0}, x_{1}$ are $Y$-complete and $x_{2}$ is not, and

- every vertex in $Y$ that is nonadjacent to $x_{2}$ has a neighbour in $A_{1}$ and is adjacent to $z$.

Then $z$ is $Y$-complete and there is a wheel $(C, Y)$ in $G$ with $x_{0}, x_{1}, z \in V(C) \subseteq\left\{x_{0}, x_{1}, z\right\} \cup A_{1}$.

Proof. If possible, choose $Y$ not satisfying the theorem, with $|Y|$ minimum. For fixed $Y$ choose $A \subseteq A_{1}$ minimal with the properties that

- $A$ is connected

- $x_{0}, x_{1}, x_{2}$ all have neighbours in $A$, and

- every vertex in $Y$ that is nonadjacent to $x_{2}$ has a neighbour in $A$.

It follows from the hypotheses that $A, Y$ are both nonempty.

(1) There exists $y \in Y$ adjacent to $z$ and with a neighbour in A, such that $Y \backslash\{y\}$ is empty or anticonnected.

For if $|Y|=1$, let $Y=\{y\}$; then since $x_{2}$ is not $Y$-complete it follows that $y$ is nonadjacent to $x_{2}$, and therefore is adjacent to $z$ and has a neighbour in $A$ and the claim holds. So assume $|Y|>1$, and choose distinct $y_{1}, y_{2} \in Y$ such that $Y \backslash\left\{y_{i}\right\}$ is anticonnected $(i=1,2)$. Not both $y_{1}, y_{2}$ is the unique nonneighbour of $x_{2}$ in $Y$; so we may assume that $x_{2}$ is not $Y \backslash\left\{y_{2}\right\}$-complete. By the minimality of $|Y|, z$ is $Y \backslash\left\{y_{2}\right\}$-complete and there is a $Y \backslash\left\{y_{2}\right\}$-complete vertex in $A$; and in particular, $y_{1}$ is adjacent to $z$ and has a neighbour in $A$, so we may set $y=y_{1}$. This proves (1).

Let $y$ be as in (1), and let $Y^{\prime}=Y \backslash\{y\}$.

(2) Either $x_{2}$ is $Y^{\prime}$-complete and nonadjacent to $y$, or $z$ is $Y$-complete and there is a path $x_{0}-p_{1}-\cdots-p_{n}-x_{1}$ from $x_{0}$ to $x_{1}$ with interior in A, containing at least two $Y^{\prime}$-complete edges.

For if $x_{2}$ is $Y^{\prime}$-complete the first assertion holds, so we assume not; and in particular $Y^{\prime}$ is nonempty. From the minimality of $|Y|, z$ is $Y^{\prime}$-complete and therefore $Y$-complete, and there is a path as in the claim. This proves (2).

(3) There is no connected $F \subseteq A$ containing neighbours of all of $x_{0}, x_{1}, x_{2}, y$ except $A$ itself.

For suppose there is. From the minimality of $A$, some member of $Y$ has no neighbour in $F$ and is nonadjacent to $x_{2}$. In particular, $x_{2}$ is not $Y^{\prime}$-complete, so $Y^{\prime}$ is nonempty and by (2), at least two vertices of $A$ are $Y^{\prime}$-complete. Since $F \neq A$, there exists $f \in A \backslash F$ such that $A \backslash\{f\}$ is connected. But every vertex in $Y \cup\left\{x_{0}, x_{1}, x_{2}\right\}$ has a neighbour in $A \backslash\{f\}$; for all members of $Y^{\prime}$ have at least two neighbours in $A$ (since $A$ contains two $Y^{\prime}$-complete vertices), and $x_{0}, x_{1}, x_{2}, y$ have neighbours in $F$. This contradicts the minimality of $A$, and therefore proves (3). 
Let $x_{0}-p_{1}-\cdots-p_{n^{-}}-x_{1}$ be a path from $x_{0}$ to $x_{1}$ with interior in $A$, and let $C$ be the hole $z-x_{0}-p_{1}-\cdots-p_{n^{-}}-x_{1}-z$.

(4) If any vertex of $p_{1}, \ldots, p_{n}$ is $Y \cup\left\{x_{2}\right\}$-complete then $z$ is $Y$-complete; and if $z$ is $Y$-complete then no edge of $x_{0}-p_{1}-\cdots-p_{n}-x_{1}$ is $Y$-complete. In particular, neither of $p_{1}, p_{n}$ is $Y \cup\left\{x_{2}\right\}$-complete.

For let $p_{i}$ be $Y \cup\left\{x_{2}\right\}$-complete, say, and suppose $z$ is not $Y$-complete. By (2), $x_{2}$ is $Y^{\prime}$-complete and nonadjacent to $y$. Let $Q$ be an antipath between $z, y$ with interior in $Y^{\prime}$, and let $R$ be an antipath between $x_{2}, p_{i}$ with interior in $\left\{x_{0}, x_{1}\right\}$. Then $z-Q-y-x_{2}-R-p_{i^{-}} z$ is an antihole, meeting the hole $C$ in at least three vertices, contrary to 15.7. This proves the first assertion. The second is immediate, for otherwise $(C, Y)$ satisfies the theorem. For the third, note that if say $p_{n}$ is $Y \cup\left\{x_{2}\right\}$-complete, then $p_{n} x_{1}$ is a $Y$-complete edge, a contradiction. This proves (4).

(5) With $p_{1}, \ldots, p_{n}$ and $C$ as in (4), if $x_{0}$ is adjacent to $x_{2}$, then $x_{2}$ is nonadjacent to all of $p_{2}, \ldots, p_{n}$.

For suppose $x_{2}$ is adjacent to one of $p_{2}, \ldots, p_{n}$, and choose $i$ with $2 \leq i \leq n$ maximum such that $x_{2}$ is adjacent to $p_{i}$. Suppose first that $i=n$. Since $x_{0}, x_{1}, p_{n}$ belong to $C$, there is no antihole of length $\geq 5$ containing them by 15.7. By (4), $p_{n}$ is not $Y$-complete, and hence there is an antipath between $p_{n}, x_{2}$ with interior in this set, and it can be completed via $x_{2}-x_{1}-x_{0}-p_{n}$ to an antihole of length $\geq 5$ containing $x_{0}, x_{1}, p_{n}$, a contradiction. So $i<n$.

Since the hole $C$ is even, it follows that $n$ is odd. From the hole $z-x_{2^{-}}-p_{i^{-}} \cdots-p_{n^{-}}-x_{1^{-}} z$ it follows that $i$ is odd. Since $i>1, x_{0}-x_{2}-p_{i^{-}} \cdots-p_{n}-x_{1}$ is an odd path of length $\geq 5$. Its ends are $Y \cup\{z\}$-complete, and its internal vertices are not, so by 13.6, $Y \cup\{z\}$ is not anticonnected. Hence $z$ is $Y$-complete. The ends of the same path are both $Y$-complete, so by 13.6, some edge of the path is $Y$-complete. Since $x_{2}$ is not $Y$-complete, this edge belongs to $C$, contrary to (4). This proves (5).

Let us choose $p_{1}, \ldots, p_{n}$ and $C$ such that either $x_{2}$ is $Y^{\prime}$-complete or $\left(C, Y^{\prime}\right)$ is a wheel (this is possible by (2)).

(6) If $x_{0}$ is adjacent to $x_{2}$, then not both $x_{2}, y$ have neighbours in $\left\{p_{1}, \ldots, p_{n}\right\}$.

For if they do, then by (5) $p_{1}$ is the only neighbour of $x_{2}$ in $\left\{p_{1}, \ldots, p_{n}\right\}$. Suppose first that $x_{2}$ is adjacent to $y$. By (2), $z$ is $Y$-complete, and $\left(C, Y^{\prime}\right)$ is a wheel, and so every vertex in $Y^{\prime}$ has a neighbour in $\left\{p_{2}, \ldots, p_{n}\right\}$. By (4) $p_{1}$ is not $Y$-complete. Therefore $z, x_{0}$ are the only $Y \cup\left\{x_{2}\right\}$ complete vertices in $C$, and by 2.10 there is a hat or a leap. Since all vertices in $Y^{\prime}$ have a neighbour in $\left\{p_{2}, \ldots, p_{n}\right\}$, and $y$ is adjacent to $x_{1}$, it follows that there is no hat, and so $y, x_{2}$ form a leap, a contradiction since they are adjacent. So $x_{2}$ is nonadjacent to $y$. Choose $j$ with $1 \leq j \leq n$ minimum such that $y$ is adjacent to $p_{j}$. From the hole $z-x_{2}-p_{1^{-}} \cdots-p_{j}-y-z$ we deduce that $j$ is odd, and therefore $x_{0}-p_{1}-\cdots-p_{j}-y-x_{0}$ is not a hole, that is, $j=1$, and hence $p_{1}$ is adjacent to $y$. By (4) $p_{1}$ is not $Y^{\prime}$-complete. If $x_{2}$ is $Y^{\prime}$-complete, then an antipath between $p_{1}$ and $y$ with interior in $Y^{\prime}$ can be extended to an antihole via $y-x_{2}-x_{1}-p_{1}$, and this antihole shares the vertices $p_{1}, x_{1}, x_{2}$ with the hole $z-x_{2}-p_{1}-\cdots-p_{n}-x_{1}-z$, contrary to 15.7. So $x_{2}$ is not $Y^{\prime}$-complete. By $(2), z$ is $Y$-complete, and $\left(C, Y^{\prime}\right)$ is a wheel. By 16.1 applied to the wheel $\left(C, Y^{\prime}\right)$ and vertex $x_{2}$, it follows that $p_{1}$ is $Y^{\prime}$-complete and therefore $Y \cup\left\{x_{2}\right\}$-complete, contrary to (4). This proves (6).

(7) Not both $x_{2}, y$ have neighbours in $\left\{p_{1}, \ldots, p_{n}\right\}$. 
For by (6) we may assume that $x_{2}$ is nonadjacent to $x_{0}$, and similarly nonadjacent to $x_{1}$. Choose $i$ with $1 \leq i \leq n$ maximum such that $x_{2}$ is adjacent to $p_{i}$. From the hole $z-x_{2}-p_{i^{-}} \cdots-p_{n^{-}} x_{1^{-}} z$ it follows that $i$ is odd. Suppose first that $x_{2}$ is not $Y^{\prime}$-complete. By $(2), z$ is $Y$-complete and $\left(C, Y^{\prime}\right)$ is a wheel. By 16.1, $p_{i}, z$ have the same wheel-parity, and so there are an odd number of $Y^{\prime}$-complete edges in $p_{i^{-}} \cdots-p_{n}-x_{1}$. By (4) no edge of the path $x_{0}-p_{1}-\cdots-p_{n}-x_{1}$ is $Y$-complete. Consequently $z x_{1}$ is the unique $Y$-complete edge of the hole $z-x_{2}-p_{i^{-}} \cdots-p_{n^{-}} x_{1}-z$ (=C $C_{1}$ say). Suppose that $y$ is nonadjacent to all $x_{2}, p_{i}, \ldots, p_{n}$. Now $y$ has a neighbour in $\left\{p_{1}, \ldots, p_{n}\right\}$ by hypothesis, so $\left\{p_{1}, \ldots, p_{n}, x_{2}\right\}(=F$ say) catches the triangle $\left\{z, x_{1}, y\right\}$. The only neighbour of $z$ in $F$ is $x_{2}$; the only neighbour of $x_{1}$ in $F$ is $p_{n}$; and $y$ is nonadjacent to both $x_{2}, p_{n}$ by assumption. By 17.1, $F$ includes a reflection of the triangle; but then $i=n$ and there is an antihole of length 6 using $z, x_{1}, p_{n}$, contrary to 15.7. This proves that $y$ is adjacent to one of $x_{2}, p_{i}, \ldots, p_{n}$. Since there is an odd number of $Y^{\prime}$-complete edges in the path $p_{i^{-}} \cdots-p_{n}-x_{1}$, it follows that every member of $Y$ is adjacent to one of $x_{2}, p_{i}, \ldots, p_{n}$. Consequently $Y$ contains no hat for $C_{1}$. Assume that $C_{1}$ has length $\geq 6$. By 2.10, $Y$ contains a leap, so there are nonadjacent $y_{1}, y_{2} \in Y$ such that $y_{1^{-}} x_{2^{-}} p_{i^{-}} \cdots-p_{n^{-}} y_{2}$ is a path, of odd length $\geq 5$. But the ends of this path are $\left\{x_{0}, x_{1}\right\}$-complete and its internal vertices are not, contrary to 13.6. So $C_{1}$ has length 4 , that is, $i=n$, and $p_{n}$ is $Y^{\prime}$-complete. By (4) it follows that $p_{n}$ is nonadjacent to $y$, and therefore $y$ is adjacent to $x_{2}$ (since we already showed that $y$ is adjacent to one of $\left.x_{2}, p_{i}, \ldots, p_{n}\right)$. From the symmetry between $x_{0}, x_{1}$ we deduce that the same holds for $p_{1}$, that is, $p_{1}$ is $Y^{\prime} \cup\left\{x_{2}\right\}$-complete and nonadjacent to $y$. Let $Q$ be an antipath between $x_{2}, y$ with interior in $Y^{\prime}$; then the three antipaths $p_{1}-x_{1}, p_{n}-x_{0}$ and $y-Q-x_{2}$ form a long prism in $\bar{G}$ with triangles $\left\{p_{1}, p_{n}, y\right\}$ and $\left\{x_{1}, x_{0}, x_{2}\right\}$, a contradiction. This proves (7) assuming that $x_{2}$ is not $Y^{\prime}$-complete.

We therefore assume that $x_{2}$ is $Y^{\prime}$-complete, and consequently nonadjacent to $y$. Now $\left\{x_{2}, p_{1}, \ldots, p_{n}\right\}$ is connected and catches the triangle $\left\{z, x_{1}, y\right\}$. By 15.7, it contains no reflection of the triangle, since as before that would give an antihole of length 6 with three vertices in $C$. So by 17.1 , there is a vertex in $\left\{x_{2}, p_{1}, \ldots, p_{n}\right\}$ with two neighbours in the triangle. The only neighbour of $z$ in it is $x_{2}$, which is nonadjacent to both $x_{1}, y$. The only neighbour of $x_{1}$ in it is $p_{n}$, and therefore $y$ is adjacent to $p_{n}$. We recall that $i$ is maximum such that $x_{2}$ is adjacent to $p_{i}$. Since $y$ is adjacent to $p_{n}$, we may choose $j$ with $i \leq j \leq n$ minimum such that $y$ is adjacent to $p_{j}$. From the hole $z-x_{2^{-}} p_{i^{-}} \cdots-p_{j}-y-z$ we

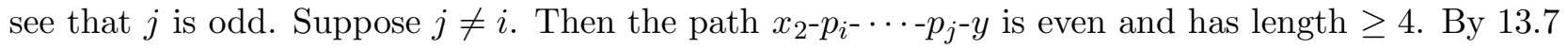
with anticonnected sets $\left\{x_{0}, x_{1}\right\}, Y^{\prime} \cup\{z\}$ we deduce that $Y^{\prime} \cup\{z\}$ is not anticonnected, and hence $z$ is $Y$-complete. Consequently, by (4), no edge of $x_{0}-p_{1^{-}} \cdots-p_{n}-x_{1}$ is $Y$-complete, and in particular $p_{n}$ is not $Y$-complete, and therefore not $Y^{\prime}$-complete (since $p_{n}$ is adjacent to $y$ ). Since there is no $Y$-complete edge in the odd path $p_{j^{-}} \cdots-p_{n}-x_{1}$, and the $Y$-complete vertex $z$ has no neighbour in its interior, it follows from 2.2 that $p_{j}$ is not $Y$-complete and hence not $Y^{\prime}$-complete. By 18.2 with sets $\left\{x_{0}, x_{1}\right\}, Y^{\prime}$, since the $\left\{x_{0}, x_{1}\right\} \cup Y^{\prime}$-complete vertex $z$ has no neighbours in $A$, it follows that there are an odd number of $Y^{\prime}$-complete edges in the path $x_{2^{2}}-p_{i^{-}} \cdots-p_{j^{-}} y$. Since $y$ is not $Y^{\prime}$-complete, they all belong to the path $x_{2}-p_{i^{-}} \cdots-p_{j}$. Since $x_{2} z, z x_{1}$ are both $Y^{\prime}$-complete edges and $x_{1} p_{n}$ is not, it follows that $p_{j}, p_{n}$ have opposite wheel-parity with respect to the wheel $\left(C_{1}, Y^{\prime}\right)$, where $C_{1}$ is $z-x_{2}-p_{i^{-}} \cdots-p_{n}-x_{1}-z$. But $p_{j}, p_{n}$ are both not $Y^{\prime}$-complete, and so $\left(C_{1}, Y^{\prime}\right)$ is an odd wheel, contrary to $G \in \mathcal{F}_{7}$. This proves that $j=i$, that is, $y$ is adjacent to $p_{i}$.

Suppose that $i<n$. If $p_{i}$ is not $Y$-complete then an antipath between $p_{i}$ and $y$ with interior in $Y^{\prime}$ can be extended via $y-x_{2}-x_{1}-p_{i}$ to an antihole sharing the vertices $p_{i}, x_{1}, x_{2}$ with the hole $z-x_{2}-p_{i^{-}} \cdots-p_{n^{-}} x_{1^{-}} z$ (=C $C_{1}$ say), contrary to 15.7. So $p_{i}$ is $Y$-complete, and therefore so is $z$, by (4). 
But then $\left(C_{1}, Y\right)$ is an odd wheel, since $z, x_{1}, p_{i}$ are $Y$-complete and $x_{2}, p_{n}$ are not (by (4)), contrary to $G \in \mathcal{F}_{7}$. So $i=n$, and hence $p_{n}$ is adjacent to both $x_{2}, y$. From the symmetry between $x_{0}, x_{1}$ it follows that $p_{1}$ is adjacent to both $x_{2}, y$. By (4), $p_{1}, p_{n}$ are not $Y$-complete. So in $\bar{G}$, the connected set $Y \cup\left\{p_{1}, p_{n}\right\}$ catches the triangle $\left\{x_{0}, x_{1}, x_{2}\right\} ; x_{0}, x_{1}, x_{2}$ all have unique neighbours in it, namely $p_{n}, p_{1}, y$ respectively; and these three vertices do not form a triangle since $y p_{1}$ is not an edge (of $\bar{G}$ ), contrary to 17.1. This proves (7).

(8) If $x_{2}$ is nonadjacent to $y$ then it is nonadjacent to both $x_{0}, x_{1}$.

For assume $x_{2}$ is nonadjacent to $y$ and adjacent to $x_{0}$ say. Now $A \cup\left\{x_{1}\right\}$ catches the triangle $\left\{z, x_{0}, x_{2}\right\}$; it contains no reflection of this triangle, since $x_{0}, x_{1}$ have no common neighbour in $A$; and the unique neighbour of $z$ in this set is nonadjacent to both $x_{0}, x_{2}$. So by 17.1 it follows that there is a vertex in $A$ adjacent to both $x_{0}, x_{2}$. Also, $A \cup x_{2}$ catches the triangle $\left\{z, x_{1}, y\right\}$. Suppose that $A \cup\left\{x_{2}\right\}$ contains a reflection of this triangle; then there exists $f \in A$ adjacent to $x_{1}, x_{2}$ and not to $y$. Since $f \in A$ it follows that $f$ is nonadjacent to $x_{0}$; but then $f-x_{2}-x_{0}-y-x_{1}-f$ is an odd hole, a contradiction. Hence by 17.1 there is a vertex in $A$ adjacent to both $x_{1}, y$. Consequently from (3), $A$ is the vertex set of a path $f_{1}-\cdots-f_{k}$, where $f_{1}$ is adjacent to $x_{0}, x_{2}$, and $f_{k}$ to $x_{1}, y$. Since $f_{1} \in A$ it follows that $f_{1}$ is not adjacent to $x_{1}$.

Now assume that $f_{1}$ is not the unique neighbour of $x_{2}$ in $A$. From (3), $f_{1}$ is the unique neighbour of $x_{0}$ in $A$. By (7), $f_{k}$ is not the unique neighbour of $x_{1}$ in $A$, and so from (3) it is the unique neighbour of $y$ in $A$. In particular $y$ is not adjacent to $f_{1}$. Both $x_{0}, z$ have unique neighbours in $A \cup\left\{x_{1}\right\}=F$ say, namely $f_{1}, x_{1}$ respectively. Now $x_{0}, z$ are both $\left\{x_{2}, y\right\}$-complete, and $f_{1}, x_{1}$ are not. Since $F \backslash\left\{x_{1}\right\}$ is connected, this contradicts 17.3. So $f_{1}$ is the unique neighbour of $x_{2}$ in $A$. Suppose that $f_{k}$ is the unique neighbour of $y$ in $A$. Then both $z, y$ have unique neighbours in $A \cup\left\{x_{2}\right\}$, namely $x_{2}, f_{k}$ respectively; and $z, y$ are $\left\{x_{0}, x_{1}\right\}$-complete, and $x_{2}, f_{k}$ are not. Once again this contradicts 17.3. So $f_{k}$ is not the unique neighbour of $y$ in $A$, and therefore it is the unique neighbour of $x_{1}$ in $F$.

Suppose that $f_{k}$ is $Y$-complete. Since $f_{k}=p_{n}$, it follows from (4) that $z$ is not $Y$-complete; and so $x_{2}$ is $Y^{\prime}$-complete by (2), and an antipath between $z, y$ with interior in $Y^{\prime}$ can be extended to an antihole via $y-x_{2}-f_{k^{-}} z$, which shares the vertices $z, x_{2}, f_{k}$ with the hole $z-x_{2^{-}} f_{1^{-}} \cdots-f_{k^{-}} x_{1^{-}} z\left(=C_{1}\right.$ say), contrary to 15.7 . So $f_{k}$ is not $Y$-complete and therefore not $Y^{\prime}$-complete (and in particular, $Y^{\prime}$ is nonempty).

Suppose that $z$ is not $Y$-complete; and therefore $Y^{\prime} \cup\{z\}$ is anticonnected, and $x_{2}$ is $Y^{\prime}$-complete by (2). Choose $h$ with $1 \leq h<k$ minimum such that $f_{h}$ is adjacent to $y$ (this exists since $f_{k}$ is not the unique neighbour of $y$ in $A$ ). The path $x_{2^{-}} f_{1^{-}} \cdots-f_{h^{-}} y$ is even, since it can be completed to a hole via $y-z-x_{2}$, and therefore the path $x_{2^{-}} f_{1^{-}} \cdots-f_{h^{-}}-y-x_{1}$ is odd (this is a path since $f_{k}$ is the unique neighbour of $x_{1}$ in $A$ ); and the ends of this path are $Y^{\prime} \cup\{z\}$-complete, and its internal vertices are not. By 13.6 it has length 3. So $f_{1}$ is adjacent to $y$ and $x_{2}$. If $f_{1}$ is not $Y^{\prime}$-complete, then an antipath between $f_{1}, y$ with interior in $Y^{\prime}$ can be completed to an antihole via $y$ - $x_{2}-x_{1}-f_{1}$, which shares the vertices $x_{1}, x_{2}, f_{1}$ with the hole $C_{1}$, contrary to 15.7 ; while if $f_{1}$ is $Y$-complete, then an antipath between $z, y$ with interior in $Y^{\prime}$ can be completed to an antihole via $y-x_{2}-x_{1^{-}} f_{1^{-}} z$, again contrary to 15.7. This proves that $z$ is $Y$-complete.

In the hole $C_{1}, z, x_{1}$ are $Y$-complete and $x_{2}, f_{k}$ are not; so since $G \in \mathcal{F}_{7}$, no other vertex of $C_{1}$ is $Y$-complete. By 2.10, $Y$ contains a leap or hat for $C_{1}$. From a hypothesis of the theorem, every 
vertex in $Y$ has a neighbour in $A \cup\left\{x_{2}\right\}$, so there is no hat, and hence there exist nonadjacent $y_{1}, y_{2}$ in $Y$ such that $y_{1}-x_{2}-f_{1^{-}} \cdots-f_{k^{-}} y_{2}$ is a path. Since both ends of this path are $\left\{x_{0}, x_{1}\right\}$-complete, and no internal vertex is $\left\{x_{0}, x_{1}\right\}$-complete, this contradicts 13.6. This proves (8).

(9) There is no connected $F \subseteq A$ containing neighbours of all of $x_{0}, x_{1}, x_{2}$ except $A$ itself.

For suppose that such a set $F$ exists with $F \neq A$, and choose $f \in A \backslash F$ such that $A \backslash\{f\}$ is connected. From the minimality of $A$, there exists $y^{\prime} \in Y$ nonadjacent to $x_{2}$ with no neighbour in $A \backslash\{f\}$, and therefore $f$ is the unique neighbour of $y^{\prime}$ in $A$. If $y^{\prime} \in Y^{\prime}$, then $x_{2}$ is not $Y^{\prime}$-complete, and therefore by (2) there are two $Y^{\prime}$-complete vertices in $A$, a contradiction. So $y^{\prime}=y$, and therefore $y$ is not adjacent to $x_{2}$. Suppose that $x_{2}$ is not adjacent to $f$. Then both $z, y$ have unique neighbours in $A \cup\left\{x_{2}\right\}$, namely $x_{2}, f ; z, y$ are $\left\{x_{0}, x_{1}\right\}$-complete, and $x_{2}, f$ are not; $f-y-z-x_{2}$ is a path; and $x_{0}, x_{1}$ both have neighbours in $A$, contrary to 17.3 . So $x_{2}$ is adjacent to $f$. By (8) $x_{2}$ is nonadjacent to both $x_{0}, x_{1}$. Since $f$ is not $\left\{x_{0}, x_{1}\right\}$-complete, we may assume from the symmetry that $f$ is nonadjacent to $x_{1}$. Now $A \cup\left\{x_{2}\right\}$ catches the triangle $\left\{z, y, x_{1}\right\}$; the only neighbour of $z$ in $A \cup\left\{x_{2}\right\}$ is $x_{2}$; the only neighbour of $y$ in $A \cup\left\{x_{2}\right\}$ is $f ; x_{2}, f$ are both nonadjacent to $x_{1}$; and so by $17.1, A \cup\left\{x_{2}\right\}$ contains a reflection of the triangle. Hence there exists $f_{1} \in A \backslash\{f\}$, adjacent to $x_{1}, x_{2}, f$ and not to $y$ (and therefore not to $x_{0}$ ). Since every path between $x_{0}, x_{1}$ with interior in $A$ has length $\geq 4$ it follows that $x_{0}$ is nonadjacent to $f, f_{1}$, and this restores the symmetry between $x_{0}, x_{1}$; and consequently by the same argument there exists $f_{0} \in A \backslash\{f\}$ adjacent to $x_{2}, f, x_{0}$ and not to $y, x_{1}$. Since $z-x_{0^{-}} f_{0^{-}} f_{1^{-}} x_{1^{-}} z$ is not an odd hole, $f_{0}$ is nonadjacent to $f_{1}$; but then $x_{0^{-}} f_{0^{-}} f-f_{1}-x_{1}$ violates $(7)$. This proves $(9)$.

From (7) and (9), it follows that there exists $f \in A$ such that $A \backslash\{f\}$ is connected, $f$ does not belong to $C$, and $f$ is the unique neighbour of $x_{2}$ in $A$.

(10) $x_{2}$ is nonadjacent to both of $x_{0}, x_{1}$.

For suppose that $x_{2}$ is adjacent to $x_{0}$ say. Suppose first that $x_{0}$ is not adjacent to $f$. Then $A \cup\left\{x_{1}\right\}$ catches the triangle $\left\{z, x_{2}, x_{0}\right\}$; the only neighbour of $z$ in $A \cup\left\{x_{1}\right\}$ is $x_{1}$; the only neighbour of $x_{2}$ in $A \cup\left\{x_{1}\right\}$ is $f ; x_{1}, f$ are both nonadjacent to $x_{0}$; and $A \cup\left\{x_{1}\right\}$ contains no reflection of the triangle since that would give a 6 -antihole with 3 vertices in common with $C$, contradicting 17.1. So $x_{0}$ is adjacent to $f$, and therefore $x_{1}$ is nonadjacent to both $x_{2}, f$. By (8) $x_{2}$ is adjacent to $y$, and therefore not $Y^{\prime}$-complete. By $(2) z$ is $Y^{\prime}$-complete and $\left(C, Y^{\prime}\right)$ is a wheel. Let $x_{2}-q_{1}-\cdots-q_{k}-x_{1}$ be a path between $x_{1}, x_{2}$ with interior in $A$ (so $f=q_{1}$ ) and let $C_{1}$ be the hole $z-x_{2}-q_{1}-\cdots-q_{k^{-}} x_{1}-z$. From (9), $A=\left\{q_{1}, \ldots, q_{k}\right\}$. Since $q_{k}=p_{n}$ and $z$ is $Y$-complete, it follows from (4) that $q_{k}$ is not $Y$-complete. Since $\left(C_{1}, Y\right)$ is not an odd wheel, it follows that $\left(C_{1}, Y\right)$ is not a wheel, and so $z, x_{1}$ are the only $Y$-complete vertices in $C_{1}$, by 2.3. By 2.10, $Y$ contains a leap or hat for $C_{1}$. But $y$ is adjacent to $x_{2}$, and all other vertices of $Y$ have at least two neighbours in $\left\{p_{1}, \ldots, p_{n}\right\}$, which is a subset of $\left\{q_{1}, \ldots, q_{k}\right\}$, a contradiction. This proves (10).

From (9) one of $x_{0}, x_{1}$ has a unique neighbour in $A$, and from the symmetry we may assume it is $x_{1}$. Let its neighbour be $f_{1}$. By (7) and (9), $x_{2}$ has no neighbour in $\left\{p_{1}, \ldots, p_{n}\right\}$, and in particular $f \neq f_{1}$. Let $Q$ be a path in $A$ between $f, f_{1}$, say $f=q_{1^{-}} \cdots-q_{k}=f_{1}$, so $z-x_{2}-q_{1^{-}} \cdots-q_{k^{-}} x_{1^{-}} z$ is a hole $\left(C_{1}\right.$ say). 
(11) $z$ is not $Y^{\prime}$-complete, and $x_{2}$ is $Y^{\prime}$-complete and nonadjacent to $y$.

For assume $z$ is $Y^{\prime}$-complete. So $z, x_{1}$ both have unique neighbours in $A \cup\left\{x_{2}\right\}$, namely $x_{2}, f_{1}$. By (4), $f_{1}$ is not $Y$-complete. So $z, x_{1}$ are $Y$-complete, and $x_{2}, f_{1}$ are not. By 17.3 , it follows that some vertex in $Y$ has no neighbour in $A$. But $y$ has a neighbour in $A$ by (1), and so some vertex in $Y^{\prime}$ has no neighbour in $A$. In particular, there is no $Y^{\prime}$-complete vertex in $A$, and so by (2), $x_{2}$ is $Y^{\prime}$-complete and nonadjacent to $y$. From 17.3 applied to the path $x_{2}-z-x_{1}-f_{1}$ and the anticonnected set $\{y\}$, it follows that $y$ is adjacent to $f_{1}$. Since $\left(C_{1}, Y\right)$ is not an odd wheel, it follows from 2.10 that $Y$ contains a leap or a hat for $C_{1}$. Since all members of $Y^{\prime}$ are adjacent to $x_{2}$ and $y$ is adjacent to $f_{1}$, there is no hat, and the leap must use $y$; so we may assume $y, y^{\prime}$ is a leap for some $y^{\prime} \in Y^{\prime}$. Hence $y-f_{1}-Q-f-x_{2}-y^{\prime}$ is a path. Since this path has odd length $\geq 5$, and its ends are $\left\{x_{0}, x_{1}\right\}$-complete and its internal vertices are not, this contradicts 13.6. So $z$ is not $Y^{\prime}$-complete. The claim follows from (2). This proves (11).

(12) $y$ is nonadjacent to all of $q_{1}, \ldots, q_{k-1}$.

For suppose not, and choose $i$ with $1 \leq i<k$ minimum such that $y$ is adjacent to $q_{i}$. From the hole $z-x_{2}-q_{1}-\cdots-q_{i}-y-z$ it follows that $i$ is odd. So by (10) $x_{2}-q_{1^{-}} \cdots-q_{i}-y-x_{1}$ is an odd path. Its ends are $Y^{\prime} \cup\{z\}$-complete, its internal vertices are not, and $Y^{\prime} \cup\{z\}$ is anticonnected by (11); so it has length 3 by 13.6, that is, $i=1$ and $y$ is adjacent to $f$. If $f$ is not $Y^{\prime}$-complete, an antipath between $f, y$ with interior in $Y^{\prime}$ can be completed to an antihole via $y-x_{2}-x_{1}-f$, sharing the vertices $x_{1}, x_{2}, f$ with $C_{1}$, contrary to 15.7. So $f$ is $Y^{\prime}$-complete. Since $z$ is not, an antipath between $z, y$ with interior in $Y^{\prime}$ can be completed to an antihole via $y-x_{2}-x_{1}-f-z$, again contrary to 15.7. This proves (12).

To conclude, $A \cup\left\{x_{2}\right\}$ catches $\left\{y, z, x_{1}\right\}$, and so by 17.1, $y$ is adjacent to $f_{1}=q_{k}$. Suppose that $x_{0}$ is adjacent to one of $q_{1}, \ldots, q_{k}$. Then $\left\{p_{1}, \ldots, p_{n}\right\} \subseteq\left\{q_{1}, \ldots, q_{k}\right\}$ from the minimality of $A$, and so the neighbours of $y$ in $C$ are precisely $x_{0}, z, x_{1}, q_{k}=p_{n}$, contrary to 2.3 applied to $C$ and $y$. So $x_{0}$ is nonadjacent to all of $q_{1}, \ldots, q_{k}$; but then $x_{2}-q_{1^{-}} \cdots-q_{k}-y$ - $x_{0}$ is an odd path of length $\geq 5$, its ends are $Y^{\prime} \cup\{z\}$-complete, and its internal vertices are not, contrary to 13.6. Thus there is no such choice of $Y$. This proves 19.2.

\section{Diamond and square wheel systems}

Now we turn to the second of the three steps of the proof of 19.1. We need two special kinds of wheel systems. Let $x_{0}, \ldots, x_{t}$ be a wheel system, and define $X_{i}, A_{i}$ as usual. Let $Y \subseteq V(G)$ be nonempty and anticonnected, such that $Y$ is disjoint from $\left\{z, x_{0}, \ldots, x_{t}\right\}$, and $x_{0}, \ldots, x_{t-1}$ are all $Y$-complete and $x_{t}$ is not. We say $x_{0}, \ldots, x_{t}$ is a

- $Y$-diamond if $t \geq 3, x_{t}$ is $X_{t-2}$-complete, and $x_{t}$ has a neighbour in $A_{t-2}$

- $Y$-square if $t \geq 3, x_{t}$ is adjacent to $x_{t-1}, x_{t}$ has no neighbour in $A_{t-2}$, and there is a vertex in $A_{t-1}$ adjacent to $x_{t}$ with a neighbour in $A_{t-2}$ 
A $Y$-diamond $x_{0}, \ldots, x_{t}$ is said to be polished if $t \geq 4, x_{t-1}$ is not $X_{t-3}$-complete, $x_{t}$ has no neighbour in $A_{t-3}, x_{t-1}$ has a neighbour in $A_{t-3}$, and there is a vertex in $A_{t-2}$ adjacent to both $x_{t}, x_{t-1}$ with a neighbour in $A_{t-3}$.

We need four lemmas to prove the main result of this section, which is the following.

20.1 Let $G \in \mathcal{F}_{7}$ and let $\left(z, A_{0}\right)$ be a frame. For all $Y \subseteq V(G) \backslash\left(A_{0} \cup\{z\}\right)$, if $Y$ is nonempty and anticonnected, and there is either a $Y$-diamond or a $Y$-square in $G$, then $z$ is $Y$-complete and $G$ contains a wheel with hub $Y$.

Proof of 20.1, assuming 20.2, 20.3, 20.4, and 20.5.

We shall prove by induction on $t$ that for any nonempty anticonnected $Y \subseteq V(G) \backslash\left(A_{0} \cup\{z\}\right)$, if there is a $Y$-diamond or $Y$-square in $G$ of height $t$, then $z$ is $Y$-complete and $G$ contains a wheel with hub $Y$. Certainly $t \geq 3$, and if $t=3$ then the result holds by 20.2 , so we may assume that $t \geq 4$. By 20.3 and 20.4, we may assume that there is an anticonnected set $Y^{\prime}$ with $Y^{\prime} \subseteq V(G) \backslash\left(A_{0} \cup\{z\}\right)$ such that either $Y \subseteq Y^{\prime}$ or $z$ is not $Y^{\prime}$-complete, and such that either:

- there is a $Y^{\prime}$-diamond in $G$ of height $t-1$, or

- there is a $Y^{\prime}$-square in $G$ of height $t-1$, or

- there is a polished $Y^{\prime}$-diamond in $G$ of height $t$.

In the first two cases, it follows from the inductive hypothesis that $z$ is $Y^{\prime}$-complete, and there is a wheel with hub $Y^{\prime}$. Since $z$ is $Y^{\prime}$-complete, it follows that $Y \subseteq Y^{\prime}$, and so $z$ is $Y$-complete and there is a wheel with hub $Y$, as required. Thus we may assume that the third case holds. By 20.2 it follows that $t \geq 5$; and by 20.5 , there is an anticonnected set $Y^{\prime \prime}$ with $Y^{\prime \prime} \subseteq V(G) \backslash\left(A_{0} \cup\{z\}\right)$ such that either $Y^{\prime} \subseteq Y^{\prime \prime}$ or $z$ is not $Y^{\prime \prime}$-complete, and either

- there is a $Y^{\prime \prime}$-diamond in $G$ of height $t-2$, or

- there is a $Y^{\prime \prime}$-square in $G$ of height $t-2$, or

- there is a polished $Y^{\prime \prime}$-diamond in $G$ of height $t-1$.

In each case it follows from the inductive hypothesis that $z$ is $Y^{\prime \prime}$-complete and there is a wheel with hub $Y^{\prime \prime}$. Consequently $Y^{\prime} \subseteq Y^{\prime \prime}$, and so $z$ is $Y^{\prime}$-complete; and therefore $Y \subseteq Y^{\prime}$, and so $z$ is $Y$-complete, and there is a wheel with hub $Y$. This proves 20.1 .

Now we turn to the proofs of the lemmas. First we show:

20.2 Let $G \in \mathcal{F}_{7}$, let $\left(z, A_{0}\right)$ be a frame, and let $Y \subseteq V(G) \backslash\left(A_{0} \cup\{z\}\right)$ be nonempty and anticonnected. There is no $Y$-square of height 3 or polished $Y$-diamond of height 4 in $G$; and if $x_{0}, \ldots, x_{3}$ is a $Y$-diamond of height 3 , then $z$ is $Y$-complete and $G$ contains a wheel $\left(C, Y \cup\left\{x_{3}\right\}\right)$.

Proof. Let $x_{0}, \ldots, x_{t}$ be a wheel system in $G$, and let $X_{i}, A_{i}$ be defined as before. Suppose first that $x_{0}, \ldots, x_{t}$ is a $Y$-square of height 3 . So $t=3, x_{3}$ is adjacent to $x_{2}, x_{3}$ has no neighbour in $A_{1}$, and there is a vertex $q$ in $A_{2}$ adjacent to $x_{3}$ with a neighbour in $A_{1}$. From the maximality of $A_{1}$ it follows that $q$ is $X_{1}$-complete, and therefore nonadjacent to $x_{2}$ (since it belongs to $A_{2}$ and so is not $X_{2}$-complete). Let $Q$ be a path from $q$ to $x_{2}$ with interior in $A_{1}$; so $Q$ has length $\geq 2$. But 
$Q$ is even since it can be completed to a hole via $x_{2}-x_{3}-q$, and so $q-Q-x_{2}-z$ is an odd path; its ends are $X_{1}$-complete, and its internal vertices are not. By 13.6 it has length 3 , and there is an antipath with interior in $X_{1}$, joining its middle vertices $\left(x_{2}\right.$ and $r$ say). This antipath can be completed via $r-z-q-x_{2}$ to an antihole of length $\geq 6$, containing $x_{0}, x_{1}$ and $z$. But let $P$ be a path from $x_{0}$ to $x_{1}$ with interior in $A_{0}$; then it has length $\geq 3$ since $A_{0}$ contains no vertex adjacent to both $x_{0}, x_{1}$, and hence $z-x_{0}-P-x_{1}-z$ is a hole of length $\geq 6$ containing $x_{0}, x_{1}$ and $z$. But this contradicts 15.7, as required.

Now suppose $x_{0}, \ldots, x_{t}$ is a polished $Y$-diamond of height 4 . So $t=4, x_{4}$ is $X_{2}$-complete, $x_{3}$ is not $X_{1}$-complete, $x_{4}$ has no neighbour in $A_{1}, x_{3}$ has a neighbour in $A_{1}$, and there is a vertex $q$ in $A_{2}$ adjacent to both $x_{4}, x_{3}$ with a neighbour in $A_{1}$. As before $q$ is $X_{1}$-complete, and therefore not adjacent to $x_{2}$; let $Q$ be a path from $q$ to $x_{2}$ with interior in $A_{1}$. The proof is completed exactly as in the previous paragraph.

So now we may assume that $x_{0}, \ldots, x_{t}$ is a $Y$-diamond of height 3 . So $t=3, x_{3}$ is $X_{1}$-complete (and therefore nonadjacent to $x_{2}$ ), and $x_{3}$ has a neighbour in $A_{1}$. But then from 19.2 with $A=A_{1}$, $v=x_{2}$ and anticonnected set $Y \cup\left\{x_{3}\right\}$, the result follows. This proves 20.2.

We remark that the pieces of this jigsaw do not seem to fit well together. There is some annoying wastage in 20.2; we produce a wheel with hub $Y \cup\left\{x_{3}\right\}$, and all we use in proving 20.1 is that there is a wheel with hub $Y$. Perhaps there is a better way to organize it, but so far it eludes us.

20.3 Let $G \in \mathcal{F}_{7}$, let $\left(z, A_{0}\right)$ be a frame, and let $Y \subseteq V(G) \backslash\left(A_{0} \cup\{z\}\right)$ be nonempty and anticonnected. Let $x_{0}, \ldots, x_{t}$ be a $Y$-diamond in $G$ of height $t \geq 4$. Suppose that there is no anticonnected set $Y^{\prime}$ with $Y \subseteq Y^{\prime} \subseteq V(G)$ such that either:

- there is a $Y^{\prime}$-diamond in $G$ of height $t-1$, or

- there is a $Y^{\prime}$-square in $G$ of height $t-1$, or

- there is a polished $Y^{\prime}$-diamond in $G$ of height $t$.

Then $z$ is $Y$-complete and $G$ contains a wheel $(C, Y)$.

Proof. Assume that either $z$ is not $Y$-complete or $G$ contains no wheel $(C, Y)$. Define $X_{i}, A_{i}$ as usual. So $x_{t}$ is $X_{t-2}$-complete, and $x_{t}$ has a neighbour in $A_{t-2}$, and $Y$ is complete to $X_{t-1}$ and not to $x_{t}$.

(1) Not both $x_{t}$ and $x_{t-1}$ have neighbours in $A_{t-3}$.

For suppose they do. If $x_{t-1}$ is $X_{t-3}$-complete, then

$$
x_{0}, \ldots, x_{t-1}
$$

is a $Y \cup\left\{x_{t}\right\}$-diamond of height $t-1$, while if $x_{t-1}$ is not $X_{t-3}$-complete, then

$$
x_{0}, \ldots, x_{t-3}, x_{t-1}, x_{t}
$$

is a $Y$-diamond of height $t-1$, in both cases a contradiction. This proves (1).

(2) There is a vertex $q$ in $A_{t-2}$ adjacent to both $x_{t}$ and $x_{t-1}$, and a path $R$ in $A_{t-2}$ from $q$ to 
$A_{t-3}$ such that not both $x_{t}$ and $x_{t-1}$ have neighbours in $A_{t-3} \cup V(R \backslash q)$.

For let $F$ be a minimal connected subgraph of $A_{t-2}$ including $A_{t-3}$ and containing neighbours of both $x_{t}$ and $x_{t-1}$. If $x_{t}, x_{t-1}$ have a common neighbour in $F$, then the claim is satisfied (from the minimality of $F$ ), so we assume not. Let $P$ be a path between $x_{t}$ and $x_{t-1}$ with interior in $F$, say $x_{t}-p_{1}-\cdots-p_{n}-x_{t-1}$. Hence $P$ has length $>2$, and the hole $z-x_{1}-P-x_{2}-z(=C$ say) it follows that $P$ is even. The only $X_{t-2}$-complete vertices in $C$ are $z$ and $x_{t}$, so by $2.10, X_{t-2}$ contains a leap or a hat for $C$. Suppose it contains a leap; then there are nonadjacent $x_{i}, x_{j} \in X_{t-2}$ such that $x_{i}-p_{1}-\cdots-p_{n}-x_{t-1}-x_{j}$ is an odd path. Since $x_{i}, x_{j}$ are $Y \cup\left\{x_{t}\right\}$-complete, it follows from 13.6 that this path contains another $Y \cup\left\{x_{t}\right\}$-complete vertex, which must be $p_{1}$ since no others are adjacent to $x_{t}$. Its ends are also $Y \cup\left\{x_{t}, z\right\}$-complete, and no internal vertex is $Y \cup\left\{x_{t}, z\right\}$-complete, so by 13.6, $Y \cup\left\{x_{t}, z\right\}$ is not anticonnected, that is, $z$ is $Y$-complete. But then let $C_{1}$ be the hole $z-x_{i}-p_{1}-\cdots-p_{n}-x_{t-1}-z$; then $\left(C_{1}, Y\right)$ is a wheel, a contradiction.

So $X_{t-2}$ contains a hat for $C$; that is, there exists $x_{i} \in X_{t-2}$ with no neighbours in $C$ except $x_{t}, z$. Hence the path $x_{i}-x_{t}-p_{1}-\cdots-p_{n}-x_{t-1}$ is odd and has length $\geq 5$, and its ends are $Y \cup\{z\}$-complete, and no internal vertex is $Y \cup\{z\}$-complete, so by 13.6, $z$ is $Y$-complete. Let $S$ be a path between $x_{i}$ and $x_{t-1}$ with interior in $F$. Then $V(S \cup P) \backslash\left\{x_{i}, x_{t}\right\}\left(=F^{\prime}\right.$ say) is connected and catches the triangle $\left\{z, x_{i}, x_{t}\right\}$. The only neighbour of $z$ in $F^{\prime}$ is $x_{t-1}$, which is nonadjacent to both $x_{i}, x_{t}$. If $F^{\prime}$ contains a reflection of the triangle, there is an antihole of length 6 containing $z, x_{t-1}, x_{t}$, which is impossible by 15.7 since these three vertices belong to $C$. So by 17.1, there is a vertex in $F^{\prime}$ adjacent to both $x_{i}, x_{t}$. Since $x_{i}$ has no neighbour in $P \backslash x_{t}$, it follows that both $x_{t}, x_{t-1}$ have neighbours in the interior of $S$, and so there is a path $P^{\prime}$ between $x_{t}, x_{t-1}$ with $P^{\prime} \backslash x_{t}$ a subpath of $S \backslash x_{i}$. As before $P^{\prime}$ has length $\geq 4$, and so $S$ has length $\geq 4$, and $P^{\prime}, S$ both have even length since they can be completed to holes through $z$. Since the $X_{t-2}$-complete vertex $z$ has no neighbours in the interior of $P^{\prime}$, from 18.2 (applied to $P^{\prime}$ with anticonnected sets $Y$ and $X_{t-2}$ ) it follows that there is a $Y$-complete edge in $P^{\prime}$, and since $x_{t}$ is not $Y$-complete, there is therefore one in $S$. But since the edges $z x_{t-1}, z x_{i}$ are also $Y$-complete, we deduce that there are at least three $Y$-complete edges in the hole $z-x_{i}-S-x_{t-1}-z$, and such that hole is the rim of a wheel with hub $Y$, a contradiction. This proves (2).

Choose $q, R$ as in (2) with $R$ minimal, and let $R$ be $r_{1}-\cdots-r_{n}$, where $r_{1}=q$ and $r_{n}$ is the only vertex of $R$ in $A_{t-3}$.

(3) $x_{t-1}$ has neighbours in $A_{t-3}$.

For assume not. Since $x_{t-1}$ has no neighbours in $A_{t-3}$ it follows that $q \notin A_{t-3}$, and so $R$ has length $>0$. Suppose first that every antipath between $x_{t-1}$ and $q$ with interior in $X_{t-2}$ is odd, and let $Q$ be such an antipath. Since all internal vertices of $Q$ have neighbours in $A_{t-3}$, and $z$ is complete to its interior and anticomplete to $A_{t-3}$, it follows from 2.2 applied in $\bar{G}$ that one end of $Q$ has a neighbour in $A_{t-3}$. By hypothesis, $x_{t-1}$ does not, so $q$ does. From the maximality of $A_{t-3}$ it follows that $q$ is $X_{t-3}$-complete; and since $q \in A_{t-2}$ and is therefore not $X_{t-2}$-complete, $q$ is nonadjacent to $x_{t-2}$. Now by assumption, every every antipath between $x_{t-1}$ and $q$ with interior in $X_{t-2}$ is odd, and so $x_{t-2}$ is adjacent to $x_{t-1}$. But then

$$
x_{0}, \ldots, x_{t-1}
$$


is a $Y \cup\left\{x_{t}\right\}$-square of height $t-1$, a contradiction. So we may assume some antipath $Q$ between between $x_{t-1}$ and $q$ with interior in $X_{t-2}$ is even.

From (2), not both $x_{t}, x_{t-1}$ have neighbours in $A_{t-3} \cup V(R \backslash q)$. Suppose that $x_{t-1}$ has such a neighbour, and so $x_{t}$ does not. Since by assumption $x_{t-1}$ has no neighbours in $A_{t-3}$, it follows that all neighbours of $x_{t-1}$ in $A_{t-3} \cup V(R \backslash q)$ lie in the interior of $R$, and in particular $R$ has length $\geq 2$. The antipath $x_{t}-x_{t-1}-Q-q$ is odd, and its ends have no neighbours in the connected set $A_{t-3} \cup\left\{r_{3}, \ldots, r_{n}\right\}$. Since $z$ is complete to its interior and anticomplete to $A_{t-3} \cup\left\{r_{3}, \ldots, r_{n}\right\}$, it follows from 2.2 applied in $\bar{G}$ that some internal vertex of this antipath has no neighbours in $A_{t-3} \cup\left\{r_{3}, \ldots, r_{n}\right\}$. But all internal vertices of $Q$ lie in $X_{t-2}$ and therefore have neighbours in $A_{t-3}$; so $x_{t-1}$ has no neighbour in $A_{t-3} \cup\left\{r_{3}, \ldots, r_{n}\right\}$. Hence $r_{2}$ is its only neighbour in $A_{t-3} \cup V(R \backslash q)$. Suppose that every antipath between $x_{t-1}$ and $r_{2}$ with interior in $X_{t-2}$ is odd, and let $Q^{\prime}$ be such an antipath. All internal vertices of $Q^{\prime}$ have neighbours in the connected set $A_{t-3}$, and $z$ is complete to the interior of $Q^{\prime}$ and anticomplete to $A_{t-3}$; so by 2.2 applied in $\bar{G}$, it follows that $r_{2}$ has neighbours in $A_{t-3}$. From the maximality of $A_{t-3}, r_{2}$ is $X_{t-3}$-complete, and therefore not adjacent to $x_{t-2}$. Since by assumption every antipath between $x_{t-1}$ and $r_{2}$ with interior in $X_{t-2}$ is odd, it follows that $x_{t-1}$ is adjacent to $x_{t-2}$. But then

$$
x_{0}, \ldots, x_{t-1}
$$

is a $Y \cup\left\{x_{t}\right\}$-square of height $t-1$, a contradiction. So some antipath $Q^{\prime}$ between $x_{t-1}$ and $r_{2}$ with interior in $X_{t-2}$ is even. Hence the antipath $x_{t-1}-Q^{\prime}-r_{2}-z$ is odd. All its internal vertices have neighbours in the connected set $A_{t-3} \cup\left\{r_{3}, \ldots, r_{n}\right\}$ and its ends do not, so by 13.6 this antipath has length 3, that is, $Q^{\prime}$ has length 2. Let $x_{i}$ be its middle vertex. Then the connected set $A_{t-3} \cup V(R \backslash$ $\left.\left\{r_{1}, r_{2}\right\}\right) \cup\left\{x_{i}, x_{t}, z\right\}$ ( $=F$ say) catches the triangle $\left\{r_{1}, r_{2}, x_{t-1}\right\}$; the only neighbours of $r_{1}$ in $F$ are $x_{t}$ and possibly $x_{i}$; the neighbours of $r_{2}$ in $F$ lie in $A_{t-3} \cup\left\{r_{3}\right\}$; and the only neighbour of $x_{t-1}$ in $F$ is $z$. This contradicts 17.1 , since $z$ has no neighbour in $A_{t-3} \cup\left\{r_{3}\right\}$.

So $x_{t-1}$ has no neighbours in $A_{t-3} \cup V(R \backslash q)$. Now the antipath $z-q-Q-x_{t-1}$ is odd, and all its internal vertices have neighbours in $A_{t-3} \cup V(R \backslash q)$, and its ends do not, so by 13.6 it has length 3 , that is, $Q$ has length 2 (let its middle vertex be $x_{i}$ ); and there is an odd path $P$ between $q, x_{i}$ with interior in $A_{t-3} \cup V(R \backslash q)$. Let $C$ be the hole $z-x_{t-1}-q-P-x_{i}-z$; then $C$ has length $\geq 6$. By 15.7 there is no antihole of length $\geq 6$ containing $q, x_{i}, x_{t-1}$. If $q$ is not $Y$-complete then an antipath between $q, x_{t}$ with interior in $Y$ can be completed to such an antihole via $x_{t}-x_{t-1}-x_{i}-q$, so $q$ is $Y$-complete; and if $z$ is not $Y$-complete, an antipath between $z$ and $x_{t}$ with interior in $Y$ can be extended to such an antihole, via $x_{t}-x_{t-1}-x_{i}-q-z$. So $z$ is also $Y$-complete. Hence the hole $C$ contains at least three $Y$-complete edges, namely $x_{i} z, z x_{t-1}$ and $x_{t-1} q$, a contradiction. This proves (3).

From (3) and the choice of $R$ it follows that $x_{t}$ has no neighbours in $A_{t-3} \cup V(R \backslash q)$. Let $Q$ be an antipath between $q$ and $x_{t-1}$ with interior in $X_{t-2}$. Then $z-q-Q-x_{t-1}-x_{t}$ is an antipath of length $\geq 4$, and its ends have no neighbours in the connected set $A_{t-3} \cup V(R \backslash q)$, and its internal vertices do, so by 13.6 it has even length, that is, $Q$ is even. The antipath $x_{t}-x_{t-1}-Q-q$ is therefore odd, and its internal vertices have neighbours in $A_{t-3}$, and $z$ is complete to its interior and anticomplete to $A_{t-3}$, so by 2.2 applied in $\bar{G}$, it follows that one of its ends, and hence $q$ has a neighbour in $A_{t-3}$. From the maximality of $A_{t-3}$ it follows that $q$ is $X_{t-3}$-complete and therefore nonadjacent to $x_{t-2}$. If $x_{t-1}$ is not $X_{t-3}$-complete, then

$$
x_{0}, \ldots, x_{t}
$$


is a polished $Y$-diamond of height $t$; while if $x_{t-1}$ is $X_{t-3}$-complete, then

$$
x_{0}, \ldots, x_{t-1}
$$

is a $Y \cup\left\{x_{t}\right\}$-diamond of height $t-1$, in both cases a contradiction. This proves 20.3.

20.4 Let $G \in \mathcal{F}_{7}$, let $\left(z, A_{0}\right)$ be a frame, and let $Y \subseteq V(G) \backslash\left(A_{0} \cup\{z\}\right)$ be nonempty and anticonnected. Let $x_{0}, \ldots, x_{t}$ be a $Y$-square in $G$ of height $t \geq 4$. Then there is a nonempty anticonnected set $Y^{\prime}$ with $Y^{\prime} \subseteq V(G) \backslash\left(A_{0} \cup\{z\}\right)$ such that either $Y=Y^{\prime}$ or $z$ is not $Y^{\prime}$-complete, and such that either:

- there is a $Y^{\prime}$-diamond in $G$ of height $t-1$, or

- there is a $Y^{\prime}$-square in $G$ of height $t-1$, or

- there is a polished $Y^{\prime}$-diamond in $G$ of height $t$.

Proof. Assume that no such $Y^{\prime}$ exists. Define $X_{i}, A_{i}$ as usual. So $x_{t}$ is adjacent to $x_{t-1}, x_{t}$ has no neighbour in $A_{t-2}$, there is a vertex $q$ in $A_{t-1}$ adjacent to $x_{t}$ with a neighbour in $A_{t-2}$, and $Y$ is complete to $X_{t-1}$ and not to $x_{t}$. From the maximality of $A_{t-2}$ it follows that $q$ is $X_{t-2}$-complete. Since $q \in A_{t-1}$, it is not $X_{t-1}$-complete, and so $q$ is nonadjacent to $x_{t-1}$.

(1) $x_{t-1}$ has neighbours in $A_{t-3}$.

For suppose not. Let $R$ be a path between $q$ and $x_{t-1}$ with interior in $A_{t-2}$. Then $R$ has length $\geq 2$, and from the hole $q-R-x_{t-1}-x_{t^{-}} q$ it follows that $R$ has even length. So the path $q-R-x_{t-1^{-}} z$ is odd, and its ends are $X_{t-2}$-complete, and its interior vertices are not, so by 13.6 it has length 3 , that is, $R$ has length 2. Let its middle vertex be $r$. Since $x_{t-1}$ has no neighbour in $A_{t-3}$, it follows that $r \in A_{t-2} \backslash A_{t-3}$. Let $Q$ be an antipath between $r$ and $x_{t-1}$ with interior in $X_{t-2}$. Since $r-Q-x_{t-1}-q-z-r$ is an antihole, it follows that $Q$ is odd. All its internal vertices have neighbours in $A_{t-3}$, and one end $x_{t-1}$ does not, and $z$ is complete to its interior and anticomplete to $A_{t-3}$. By 2.2 applied in $\bar{G}$, it follows that $r$ has neighbours in $A_{t-3}$. Hence $r$ is $X_{t-3}$-complete, and nonadjacent to $x_{t-2}$. Since $z-x_{t-1}-r-q-x_{t-2^{-}} z$ is not an odd hole it follows that $x_{t-2}$ is adjacent to $x_{t-1}$. But then

$$
x_{0}, \ldots, x_{t-1}
$$

is a $\{q\}$-square of height $t-1$, and yet $z$ is not $\{q\}$-complete, a contradiction. This proves (1).

(2) $q$ has neighbours in $A_{t-3}$.

For suppose not. Let $S$ be an antipath between $x_{t}$ and $x_{t-1}$ with $V(S) \subseteq X_{t}$, that is, with interior in $X_{t-2}$. Then $x_{t}-S-x_{t-1}-q$ is an antipath with length $\geq 3$; by (1), all its internal vertices have neighbours in $A_{t-3}$, and its ends do not, and $z$ is complete to its interior and anticomplete to $A_{t-3}$; so by 2.2 applied in $\bar{G}$ it follows that $S$ has odd length. But then $x_{t}-S-x_{t-1}-q-z$ has odd length $\geq 5$, and its internal vertices have neighbours in $A_{t-2}$ and its ends do not, contrary to 13.6 applied in $\bar{G}$. This proves (2). 
If $x_{t-1}$ is $X_{t-3}$-complete, then

$$
x_{0}, \ldots, x_{t-1}
$$

is a $\{q\}$-diamond of height $t-1$, and yet $z$ is not $\{q\}$-complete, a contradiction. So $x_{t-1}$ is not $X_{t-3}$-complete. It follows from (2) that if $x_{t}$ is $X_{t-3}$-complete then

$$
x_{0}, \ldots, x_{t-3}, x_{t-1}, x_{t-2}, x_{t}
$$

is a polished $Y$-diamond of height $t$, while if $x_{t}$ is not $X_{t-3}$-complete then

$$
x_{0}, \ldots, x_{t-3}, x_{t-1}, x_{t}
$$

is a $Y$-square of height $t-1$, in either case a contradiction. This proves 20.4 .

20.5 Let $G \in \mathcal{F}_{7}$, let $\left(z, A_{0}\right)$ be a frame, and let $Y \subseteq V(G) \backslash\left(A_{0} \cup\{z\}\right)$ be nonempty and anticonnected. Let $x_{0}, \ldots, x_{t+1}$ be a polished $Y$-diamond in $G$ of height $t+1 \geq 5$. Then there is a nonempty anticonnected set $Y^{\prime}$ with $Y^{\prime} \subseteq V(G) \backslash\left(A_{0} \cup\{z\}\right)$ such that either $Y \subseteq Y^{\prime}$ or $z$ is not $Y^{\prime}$-complete, and such that either:

- there is a $Y^{\prime}$-diamond in $G$ of height $t-1$, or

- there is a $Y^{\prime}$-square in $G$ of height $t-1$, or

- there is a polished $Y^{\prime}$-diamond in $G$ of height $t$.

Proof. Suppose that no such $Y^{\prime}$ exists. Let $x_{0}, \ldots, x_{t+1}$ be a polished $Y$-diamond in $G$, and define $X_{i}, A_{i}$ as usual. So $x_{t+1}$ is $X_{t-1}$-complete, $x_{t}$ is not $X_{t-2}$-complete, $x_{t+1}$ has no neighbour in $A_{t-2}$, $x_{t}$ has a neighbour in $A_{t-2}$, there is a vertex $q$ in $A_{t-1}$ adjacent to both $x_{t+1}, x_{t}$ with a neighbour in $A_{t-2}$, and $Y$ is complete to $X_{t}$ and not to $x_{t+1}$. From the maximality of $A_{t-2}$ it follows that $q$ is $X_{t-2}$-complete, and therefore nonadjacent to $x_{t-1}$.

Choose a path $v_{1}-\cdots-v_{s}$ with $s$ minimum such that $v_{1}, \ldots, v_{s} \in A_{t-2}$, and $v_{1}$ is adjacent to $q$, and $v_{s} \in A_{t-3}$. (If $q$ has a neighbour in $A_{t-3}$ then $s=1$.) Let $R$ be a path between $q$ and $x_{t-1}$ with interior in $A_{t-2}$, and if possible with interior in $A_{t-3} \cup\left\{v_{1}, \ldots, v_{s}\right\}$. Then $R$ has length $\geq 2$, and from the hole $q-R-x_{t-1^{-}} x_{t+1^{-}} q$ it follows that $R$ has even length. So the path $q-R-x_{t-1^{-}} z$ is odd, and its ends are $X_{t-2}$-complete, and its internal vertices are not, so by 13.6 it has length 3 , that is, $R$ has length 2 . Let its middle vertex be $r$.

(1) $x_{t-1}$ has neighbours in $A_{t-3}$.

For suppose not. It follows that $r \in A_{t-2} \backslash A_{t-3}$. Let $Q$ be an antipath between $r$ and $x_{t-1}$ with interior in $X_{t-2}$. Since $r-Q-x_{t-1}-q-z-r$ is an antihole, it follows that $Q$ is odd. All its internal vertices have neighbours in $A_{t-3}$, and one end $x_{t-1}$ does not, and $z$ is complete to its interior and anticomplete to $A_{t-3}$. By 2.2 applied in $\bar{G}$, it follows that $r$ has neighbours in $A_{t-3}$. Hence $r$ is

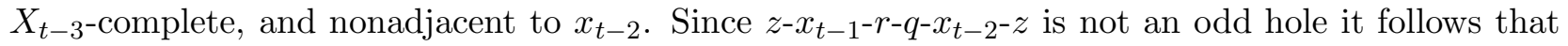
$x_{t-2}$ is adjacent to $x_{t-1}$. But then

$$
x_{0}, \ldots, x_{t-1}
$$

is a $\{q\}$-square of height $t-1$, and yet $z$ is not $\{q\}$-complete, a contradiction. This proves (1). 
From (1) it follows that it is possible to choose $R$ with interior in $A_{t-3} \cup\left\{v_{1}, \ldots, v_{s}\right\}$, and therefore we have done so.

(2) $q$ has neighbours in $A_{t-3}$, and therefore $r \in A_{t-3}$.

For suppose it does not. Then $s \geq 2$ and $r=v_{1}$. Let $Q$ be an antipath between $x_{t-1}$ and $r$ with interior in $X_{t-2}$. From the antihole $x_{t-1}-Q-r-z-q-x_{t-1}$ it follows that $Q$ is odd. Hence the antipath $q-x_{t-1}-Q-r-x_{t+1}$ is odd with length $\geq 5$; and its internal vertices have neighbours in $A_{t-3} \cup\left\{v_{2}, \ldots, v_{s}\right\}$, and its ends do not, contrary to 13.6 applied in $\bar{G}$. This proves (2).

(3) $x_{t-1}$ is not $X_{t-3}$-complete.

For if it is, then

$$
x_{0}, \ldots, x_{t-1}
$$

is a $\{q\}$-diamond of height $t-1$, and yet $z$ is not $\{q\}$-complete, a contradiction. This proves (3).

(4) $x_{t}$ has no neighbour in $A_{t-3}$.

For suppose $x_{t}$ has a neighbour in $A_{t-3}$. If $x_{t}$ is $X_{t-3}$-complete then since it is not $X_{t-2}$-complete, it is nonadjacent to $x_{t-2}$, and therefore

$$
x_{0}, \ldots, x_{t-2}, x_{t}
$$

is a $Y \cup\left\{x_{t+1}\right\}$-diamond of height $t-1$; while if $x_{t}$ is not $X_{t-3}$-complete then

$$
x_{0}, \ldots, x_{t-3}, x_{t-1}, x_{t}, x_{t+1}
$$

is a polished $Y$-diamond of height $t$, in either case a contradiction. This proves (4).

In particular, $x_{t}$ is not adjacent to $r$. Since $z-x_{t}-q-r-x_{t-1^{-}} z$ is not an odd hole it follows that $x_{t}$ is adjacent to $x_{t-1}$. If $x_{t}$ is $X_{t-3}$-complete, then

$$
x_{0}, \ldots, x_{t-3}, x_{t-1}, x_{t-2}, x_{t}
$$

is a polished $Y \cup\left\{x_{t+1}\right\}$-diamond of height $t$; while if $x_{t}$ is not $X_{t-3}$-complete, then

$$
x_{0}, \ldots, x_{t-3}, x_{t-1}, x_{t}
$$

is a $Y \cup\left\{x_{t+1}\right\}$-square of height $t-1$, in either case a contradiction. This proves 20.5.

\section{From wheel systems to wheels}

Now we complete the proof of 19.1. First we need a lemma.

21.1 Let $G \in \mathcal{F}_{7}$, and let $X, Y$ be disjoint nonempty anticonnected subsets of $V(G)$, complete to each other. Let $p_{1}, \ldots, p_{n}$ be a path in $G \backslash(X \cup Y)$ of length $\geq 4$, such that $p_{1}, p_{n}$ are $X$-complete and $p_{2}, \ldots, p_{n-1}$ are not. Suppose that either: 
1. $p_{1}, p_{2}, p_{3}$ are $Y$-complete, or

2. there exists $i$ with $1 \leq i \leq n-3$ such that $p_{i}, p_{i+1}, p_{i+2}, p_{i+3}$ are all $Y$-complete, or

3. there exists $i$ with $1 \leq i \leq n-3$ such that $p_{i+1}, p_{i+2}$ are $Y$-complete and $p_{i}, p_{i+3}$ are not.

Then there is a wheel in $G$ with hub $Y$.

Proof. In the second and third case let $i$ be as given, and in the first case let $i=1$. Let $Q$ be an antipath joining $p_{i+1}, p_{i+2}$ with interior in $X$. Since $1<i+1, i+2<n$, and $n \geq 5$, and $p_{1}, p_{n}$ are both complete to the interior of $Q$, it follows from 15.4 that $Q$ has length 2, that is, there exists $x \in X$ nonadjacent to both $p_{i+1}, p_{i+2}$. Choose $h$ with $1 \leq h \leq i$ maximum such that $x$ is adjacent to $p_{h}$, and choose $j$ with $i+3 \leq j \leq n$ minimum such that $x$ is adjacent to $p_{j}$. Then $x-p_{h^{-}} \cdots-p_{j}-x$ is a hole of length $\geq 6$, say $C$, and $x, p_{i}, p_{i+1}, p_{i+2}, p_{i+3}$ are all vertices of it, and $x, p_{i+1}, p_{i+2}$ are $Y$-complete. In the first case $x p_{1}, p_{1} p_{2}, p_{2} p_{3}$ are all $Y$-complete edges of the hole, so $(C, Y)$ is a wheel. In the second case, the three edges of $p_{i}-p_{i+1}-p_{i+2}-p_{i+3}$ are all $Y$-complete edges of $C$, so again $(C, Y)$ is a wheel. In the third case, 2.3 implies that $(C, Y)$ is a wheel (and in this case it is in fact an odd wheel, a contradiction). This proves 21.1.

The final step of the proof of 19.1 is given by the following. (In this paper we only apply it to graphs in containing no pseudowheels, that is, graphs in $\mathcal{F}_{8}$, so the first hypothesis could be simplified; but it is convenient to present it this way for a future application.)

21.2 Let $G \in \mathcal{F}_{7}$, and let $Y \subseteq V(G)$, such that there do not exist $X, P$ so that $(X, Y, P)$ is a pseudowheel. Let $\left(z, A_{0}\right)$ be a frame, and let $x_{0}, \ldots, x_{t+1}$ be a wheel system with hub $Y$, and with $t \geq 2$. Define $X_{i}, A_{i}$ as usual. Suppose that $x_{t+1}$ has no neighbour in $A_{t-1}$; and moreover that at most one member of $Y$ has no neighbour in $A_{t-1} \cup\left\{x_{t+1}\right\}$, and any such vertex has a neighbour in $A_{t}$. Then there is a wheel in $G$ with hub $Y$.

\section{Proof.}

(1) There do not exist $x_{i}, x_{j} \in X_{t}$ joined by an odd path $x_{i}-x_{t+1}-P-x_{j}$ of length $\geq 5$ such that $x_{i}, x_{j} \in X_{t}$ and $P$ has interior in $A_{t}$.

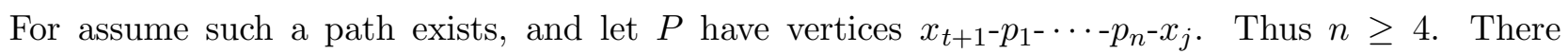
is an even path $S$ between $x_{i}$ and $x_{j}$ with interior in $A_{t-1}$. Since $x_{i}-x_{t+1}-P-x_{j}-S-x_{i}$ is not an odd hole, and $x_{t+1}$ has no neighbours in $A_{t-1}$, it follows that $\left\{p_{1}, \ldots, p_{n}\right\} \cup A_{t-1}$ is connected. Since $p_{1} \notin A_{t-1}$, there exists $k$ such that $p_{k} \notin A_{t-1}$ and $p_{k}$ has a neighbour in $A_{t-1}$; and since $p_{k}$ is not adjacent to $z$, it follows from the maximality of $A_{t-1}$ that $p_{k}$ is $X_{t-1}$-complete. Since at least one of $x_{i}, x_{j}$ is in $X_{t-1}$, it follows that $k=n$ and $i=t$. But $\left\{p_{1}, \ldots, p_{n}, x_{j}\right\} \cup A_{t-1}(=F$ say) catches the triangle $\left\{z, x_{t+1}, x_{t}\right\}$; the only neighbour of $z$ in $F$ is $x_{j}$; the only neighbour of $x_{t+1}$ in $F$ is $p_{1}$; and $x_{j}, p_{1}$ are nonadjacent (since $n \geq 4$ ), and are both nonadjacent to $x_{t}$, contrary to 17.1. This proves (1).

Since $x_{t+1}$ has a neighbour in $A_{t}$ and none in $A_{t-1}$, there is a path from $x_{t+1}$ to $A_{t-1}$ with interior in $A_{t} \backslash A_{t-1}$. Hence there is a path $x_{t+1}-p_{1}-\cdots-p_{m}$ such that $p_{1}, \ldots, p_{m} \in A_{t} \backslash A_{t-1}$ and $p_{m}$ is the unique vertex of this path with a neighbour in $A_{t-1}$. (Hence $m \geq 1$, and $p_{m}$ is 
$X_{t-1}$-complete.) Choose such a path such that if possible, every member of $Y$ has a neighbour in $A_{t-1} \cup\left\{x_{t+1}, p_{1}, \ldots, p_{m}\right\}$.

(2) We may assume that one of $x_{0}, \ldots, x_{t}$ is nonadjacent to both $x_{t+1}, p_{1}$.

For certainly there is an antipath $Q$ joining $x_{t+1}, p_{1}$ with interior in $X_{t}$, since $x_{t+1}, p_{1}$ are not $X_{t^{-}}$ complete. Suppose that $Q$ is odd. Every vertex of the interior of $Q$ has neighbours in the connected set $A_{t-1}$, and $x_{t+1}$ does not, and $z$ is complete to the interior of $Q$ and anticomplete to $A_{t-1}$; so by 2.2 applied in $\bar{G}$ it follows that $p_{1}$ has a neighbour in $A_{t-1}$. Hence $m=1$, and $p_{1}$ is $X_{t-1}$-complete, and therefore not adjacent to $x_{t}$. If $x_{t}$ is also nonadjacent to $x_{t+1}$ then the claim holds, and if $x_{t}$ is adjacent to $x_{t+1}$, then

$$
x_{0}, \ldots, x_{t+1}
$$

is a $Y$-square, and the theorem holds by 20.1. Now assume that $Q$ is even. The antipath $z-p_{1}-Q-x_{t+1}$ is therefore odd and has length $\geq 3$; all its internal vertices have neighbours in the connected set $A_{t-1} \cup\left\{p_{2}, \ldots, p_{m}\right\}$, and its ends do not. So it has length 3, by 13.6 applied in $\bar{G}$, and hence $Q$ has length 2. This proves (2).

(3) Every vertex in $Y$ has a neighbour in $A_{t-1} \cup\left\{x_{t+1}, p_{1}, \ldots, p_{m}\right\}$.

For suppose some $y \in Y$ has no such neighbour. By hypothesis $y$ has a neighbour in $A_{t}$. Consequently there is a connected subset $F$ of $A_{t}$ including $A_{t-1} \cup\left\{p_{1}, \ldots, p_{m}\right\}$ which contains a neighbour of $y$, and we may choose $F$ minimal with this property. Since $y$ has no neighbour in $A_{t-1} \cup\left\{p_{1}, \ldots, p_{m}\right\}$, it follows from the minimality of $F$ that $y$ has a unique neighbour in $F$, say $f$, and therefore $f \in A_{t} \backslash A_{t-1}$. There is a path $R$ between $y$ and $x_{t+1}$ with interior in $F$, and therefore $z-x_{t+1}-R-y-z$ is a hole $(C$ say), and so $R$ has even length. Suppose it has length $\geq 4$. The only $X_{t}$-complete vertices in $C$ are $z, y$, so by $2.10, X_{t}$ contains a hat or leap. By (1) there is no leap, so there exists $x \in X_{t}$ with no neighbours in $C$ except $y, z$. But $F \cup\left\{x_{t+1}\right\}$ catches the triangle $\{x, y, z\}$; the only neighbour of $z$ in $F \cup\left\{x_{t+1}\right\}$ is $x_{t+1}$; the only neighbour of $y$ in $F \cup\left\{x_{t+1}\right\}$ is $f$; and $x_{t+1}, f$ are nonadjacent, and both nonadjacent to $x$, contrary to 17.1. So $R$ has length 2 , and therefore $x_{t+1}$ is adjacent to $f$.

Since $y$ has no neighbour in $\left\{x_{t+1}\right\} \cup A_{t-1}$, it follows from the hypothesis that all other members of $Y$ have neighbours in $\left\{x_{t+1}\right\} \cup A_{t-1}$. We recall that initially we chose the path $x_{t+1}-p_{1^{-}} \cdots-p_{m}$ such that $p_{m}$ is the unique vertex of it with a neighbour in $A_{t-1}$, and if possible every member of $Y$ has a neighbour in $A_{t-1} \cup\left\{x_{t+1}, p_{1}, \ldots, p_{m}\right\}$. Since $f$ is adjacent to both of $y, x_{t+1}$, it follows that $f$ has no neighbours in $A_{t-1}$, and $f$ is nonadjacent to $p_{2}, \ldots, p_{m}$, since otherwise there would be a better choice of path using $f$. Let $Q$ be an antipath between $f, x_{t+1}$ with interior in $X_{t}$. Every internal vertex of $Q$ has a neighbour in $A_{t-1}$, and its ends do not, and $z$ is complete to the interior of $Q$ and anticomplete to $A_{t-1}$; so by 2.2 applied in $\bar{G}$, it follows that $Q$ is even. So the antipath $y-x_{t+1}-Q-f$ is odd, and all its internal vertices have neighbours in $A_{t-1} \cup\left\{p_{1}, \ldots, p_{m}\right\}$, and $y$ does not; and $z$ is complete to the interior of the antipath and anticomplete to $A_{t-1} \cup\left\{p_{1}, \ldots, p_{m}\right\}$. By 2.2 applied in $\bar{G}$, it follows that $f$ has a neighbour in $A_{t-1} \cup\left\{p_{1}, \ldots, p_{m}\right\}$, and therefore $f$ is adjacent to $p_{1}$. By (2) there exists $x \in X_{t}$ nonadjacent to $x_{t+1}, p_{1}$. Consequently, $\left\{z, y, x, p_{2}, \ldots, p_{m}\right\} \cup A_{t-1}$ (= $F^{\prime}$ say) catches the triangle $\left\{x_{t+1}, f, p_{1}\right\}$. The only neighbour of $x_{t+1}$ in $F^{\prime}$ is $z$; the only neighbours of $f$ in $F^{\prime}$ are $y$ and possibly $x$; and $x, y, z$ are all nonadjacent to $p_{1}$. By $17.1, F^{\prime}$ contains a reflection of the triangle, and hence there is a vertex in $F^{\prime}$ adjacent to both of $z, p_{1}$. But the only neighbours of $z$ in 
$F^{\prime}$ are $x, y$, and they are both nonadjacent to $p_{1}$, a contradiction. This proves (3).

Since $p_{m}$ is $X_{t-1}$-complete it follows that $x_{0}, \ldots, x_{t-1}$ all have neighbours in $p_{1}, \ldots, p_{m}$. Since $x_{t}, p_{m}$ have neighbours in $A_{t-1}$ and none of $x_{t+1}, p_{1}, \ldots, p_{m-1}$ have neighbours in $A_{t-1}$, we can extend the path $x_{t+1}-p_{1}-\cdots-p_{m}$ to a path $x_{t+1}-p_{1}-\cdots-p_{m}-p_{m+1}-\cdots-p_{n}$ containing neighbours of all members of $X_{t}$. By (2), we can choose $i$ with $2 \leq i \leq n$ maximum such that some vertex of $X_{t}$ is nonadjacent to all of $x_{t+1}, p_{1}, \ldots, p_{i-1}$; and choose $s$ with $0 \leq s \leq t$ such that $x_{s}$ is nonadjacent to all of $x_{t+1}, p_{1}, \ldots, p_{i-1}$. Since every vertex in $X_{t}$ has a neighbour in $\left\{x_{t+1}, p_{1}, \ldots, p_{n}\right\}$, it follows from the maximality of $i$ that every vertex in $X_{t}$ is adjacent to one of $x_{t+1}, p_{1}, \ldots, p_{i}$, and in particular, $x_{s}$ is adjacent to $p_{i}$. Note that if $i>m$ then $s=t$, since $p_{m}$ is $X_{t-1}$-complete.

(4) $i$ is odd, and $p_{i}$ is $Y$-complete.

For $z-x_{t+1}-p_{1}-\cdots-p_{i}-x_{s^{-}} z$ is a hole $C$ say, and so $i$ is odd. Suppose $p_{i}$ is not $Y$-complete. Now $C$ has length $\geq 6$, and $z, x_{s}$ are $Y$-complete (since $Y$ is a hub), and $x_{t+1}, p_{i}$ are not. Since $(C, Y)$ is not an odd wheel, 2.10 implies that $Y$ contains a leap or hat for $C$. Suppose it contains a leap; then there are nonadjacent $y_{1}, y_{2} \in Y$ such that $y_{1}-x_{t+1}-p_{1}-\cdots-p_{i}-y_{2}$ is a path. This path is odd and has length $\geq 5$, and its ends are $X_{t}$-complete and its internal vertices are not, contrary to 13.6. So $Y$ contains a hat, that is, there exists $y \in Y$ nonadjacent to $x_{t+1}, p_{1}, \ldots, p_{i}$. By (3), $y$ has a neighbour in $A_{t-1} \cup\left\{p_{j}: i+1 \leq j \leq m\right\}$.

Suppose first that $i \leq m$, and let $p_{i}-r_{1^{-}} \cdots-r_{k}-y$ be a path from $p_{i}$ to $y$ with interior in $A_{t-1} \cup$ $\left\{p_{i+1}, \ldots, p_{m}\right\}$. Then $z-x_{t+1}-p_{1^{-}} \cdots-p_{i}-r_{1^{-}} \cdots-r_{k}-y-z$ is a hole of length $\geq 6$, and the only $X_{t}$-complete vertices in this hole are $z, y$. Since this hole is not the rim of an odd wheel, 2.10 implies that $X$ contains a hat or leap, and so some $x \in X_{t}$ has no neighbour in $\left\{x_{t+1}, p_{1}, \ldots, p_{i}\right\}$, contrary to the choice of $i$.

Now suppose that $i>m$, and so $s=t$. Let $p_{m^{-}} r_{1^{-}} \cdots-r_{k^{-}} y$ be a path from $p_{m}$ to $y$ with interior in $A_{t-1}$. Again, $z-x_{t+1}-p_{1^{-}} \cdots-p_{m^{-}}-r_{1^{-}} \cdots-r_{k^{-}} y-z$ is a hole of length $\geq 6$, and its only $X_{t}$-complete vertices are $z, y$. By $2.10 X_{t}$ contains a hat or leap. By (1) it contains no leap, so there exists $x \in X_{t}$ nonadjacent to all $x_{t+1}, p_{1}, \ldots, p_{m}, r_{1}, \ldots, r_{k}$. Since $p_{m}$ is $X_{t-1}$-complete, it follows that $x=x_{t}$. Now $\left\{x_{t+1}, p_{1}, \ldots, p_{i}, r_{1}, \ldots, r_{k}\right\}$ ( $=F$ say) is connected, and catches the triangle $\left\{y, z, x_{t}\right\}$; the only neighbour of $z$ in $F$ is $x_{t+1}$; the only neighbour of $y$ in $F$ is $r_{k}$ (because $y$ is nonadjacent to $x_{t+1}, p_{1}, \ldots, p_{i}$ ); and the only neighbour of $x_{t}$ in $F$ is $p_{i}$ (because $x_{t}$ is a hat). Since $x_{t+1}$ is not adjacent to $p_{i}$, this contradicts 17.1 . This proves (4).

(5) Let $R$ be a path from $x_{t}$ to some vertex $r$, such that $r$ is the unique $X_{t-1}$-complete vertex in $R$, and $V\left(R \backslash x_{t}\right) \subseteq A_{t-1} \cup\left\{p_{1}, \ldots, p_{m}\right\}$. Then $R$ is odd, and has length $\geq 3$. In particular, $x_{t}$ is nonadjacent to $p_{m}, p_{m-1}$.

For assume that $R$ is even. Then the path $z-x_{t}-R-r$ is odd, and its ends are $X_{t-1}$-complete, and its internal vertices are not, so by 13.6, it has length 3 , that is, $R$ has length 2 . Let $q$ be the middle vertex of $R$. By 13.6 there is an odd antipath $Q$ joining $q, x_{t}$ with interior in $X_{t-1}$. Now $p_{m}$ is $X_{t-1}$-complete and nonadjacent to $x_{t}$, and since $Q$ cannot be completed to an antihole via $x_{t}-p_{m}-q$, it follows that $p_{m}$ is adjacent to $q$. Suppose first that $q \in\left\{p_{1}, \ldots, p_{m}\right\}$; then it follows that $q=p_{m-1}$. Hence $q-Q-x_{t}-p_{m}$ is an even antipath of length $\geq 4 ; q$ is its only vertex that is anticomplete to $A_{t-1}$, and $p_{m}$ is its only vertex that is anticomplete to $\left\{z, x_{t+1}, p_{1}, \ldots, p_{m-2}\right\}$. Since the sets $A_{t-1},\left\{z, x_{t+1}, p_{1}, \ldots, p_{m-2}\right\}$ are 
each connected and anticomplete to each other, this contradicts 13.7 applied in $\bar{G}$. So $q \in A_{t-1}$, and in particular $x_{t}$ is nonadjacent to $p_{m}, p_{m-1}$. Let $R^{\prime}$ be a path between $x_{t}, p_{m}$ with interior in $\left\{z, x_{t+1}, p_{1}, \ldots, p_{m}\right\}$; then $x_{t}-R-p_{m}-R^{\prime}-x_{t}$ is a hole of length $\geq 6$ sharing the vertices $x_{t}, q, p_{m}$ with the antihole $q-Q-x_{t}-p_{m}-z-q$, contrary to 15.7 . So $R$ is odd. Since $r$ is not $X_{t}$-complete, it follows that $R$ has length $\geq 3$. The last assertion of the claim is immediate. This proves (5).

(6) We may assume that none of $x_{t+1}, p_{1}, \ldots, p_{i-1}$ is $X_{t-1}$-complete, and in particular $i \leq m$.

For suppose first that one of $p_{1}, \ldots, p_{i-1}$ is $X_{t-1}$-complete, and choose $h$ with $1 \leq h<i$ maximum such that $p_{h}$ is $X_{t-1}$-complete. Since $p_{h}$ is not adjacent to $x_{s}$ it follows that $s=t$, and therefore $p_{i}$ is not $X_{t-1}$-complete (because $p_{i}$ is not $X_{t}$-complete and is adjacent to $x_{s}$ ). By (5), $i-h$ is even, and so the path $p_{h^{-}} \cdots-p_{i^{-}} x_{t^{-}} z$ is even and has length $\geq 4$. Since its only $X_{t-1^{-c o m p l e t e ~ v e r t i c e s ~ a r e ~}}$ its ends, and since $z, x_{t}, p_{i}$ are $Y$-complete by (4), it follows from 21.1 that there is a wheel with hub $Y$, and the theorem holds. So we may assume that none of $p_{1}, \ldots, p_{i-1}$ is $X_{t-1}$-complete, and in particular $i \leq m$, since $p_{m}$ is $X_{t-1}$-complete. Now assume that $x_{t+1}$ is $X_{t-1}$-complete. Since $x_{t+1}$ is nonadjacent to $x_{s}$ it follows that $s=t$. Let $R$ be a path between $x_{t}, p_{m}$ with interior in $A_{t-1}$. By (5), $R$ is odd, and so the path $x_{t+1}-p_{1}-\cdots-p_{i}-x_{t}-R-p_{m}$ is odd, of length $\geq 5$, its ends are $X_{t-1}$-complete, and its internal vertices are not, contrary to 13.6. This proves (6).

Choose $k$ with $i \leq k \leq m$ minimum such that $p_{k}$ is $X_{t-1 \text {-complete. }}$

(7) None of $x_{t+1}, p_{1}, \ldots, p_{k-1}$ is $X_{t-1}$-complete, and $k$ is odd.

The first assertion follows from (6) and the choice of $k$. Hence the path $z-x_{t+1}-p_{1}-\cdots-p_{k}$ has length $\geq 4$, and its ends are $X_{t-1}$-complete, and its internal vertices are not; so by 13.6, it has even length. This proves (7).

(8) $x_{t}$ is adjacent to one of $p_{1}, \ldots, p_{k}$.

For suppose $x_{t}$ is nonadjacent to all of $p_{1}, \ldots, p_{k}$. From the definition of $i$ it follows that $x_{t}$ is adjacent to $x_{t+1}$. Let $S$ be a path between $x_{t}, p_{k}$ with interior in $A_{t-1} \cup\left\{p_{k+1}, \ldots, p_{m}\right\}$, and let $C$ be the hole $x_{t}-x_{t+1}-p_{1}-\cdots-p_{k}-S-x_{t}$. Since $C$ is even and $k$ is odd, it follows that $S$ is even, and so by (5), some internal vertex of $S$ is $X_{t-1}$-complete. The path $z-x_{t}-S-p_{k}$ is odd, and its ends are $X_{t-1}$-complete, so by 2.3 it contains an odd number of $X_{t-1}$-complete edges. Since $x_{t}$ is not $X_{t-1}$-complete, all these $X_{t-1}$-complete edges belong to $S$ and hence to $C$, and there are no further $X_{t-1}$-complete edges in $C$. Thus an odd number of edges of $C$ are $X_{t-1}$-complete, and so by 2.3 there is exactly one, and exactly two $X_{t-1}$-complete vertices. Since $p_{k}$ is $X_{t-1}$-complete, the second such vertex is the neighbour of $p_{k}$ in $S$. This therefore does not belong to $A_{t-1}$, and so $k<m$, and $p_{k+1}$ is the second $X_{t-1}$-complete vertex of $C$. By 2.10 applied to $C, X_{t-1}$ contains a leap or hat, and in either case some $x \in X_{t-1}$ is nonadjacent to all of $x_{t}, x_{t+1}, p_{1}$, and adjacent to $p_{k}$. Hence $\left(V(C) \backslash\left\{x_{t}, x_{t+1}\right\}\right) \cup\{x\}$ (=F say) catches the triangle $\left\{z, x_{t}, x_{t+1}\right\}$; the only neighbour of $z$ in $F$ is $x$; the only neighbour of $x_{t+1}$ in $F$ is $p_{1}$; and $x, p_{1}$ are nonadjacent, and are both nonadjacent to $x_{t}$, contrary to 17.1 . This proves $(8)$.

(9) $p_{k}$ is $Y$-complete. 
For suppose not. Then $i<k$, by (4). But then $z, X_{t-1}$ are $Y$-complete and $x_{t+1}, p_{k}$ are not, and some vertex of the path $x_{t+1}-p_{1}-\cdots-p_{k}$ is $Y$-complete (namely $\left.p_{i}\right)$; and so $\left(X_{t-1}, Y, z-x_{t+1}-p_{1}-\cdots-p_{k}\right)$ is a pseudowheel, contrary to $G \in \mathcal{F}_{8}$. This proves (9).

By (8), we may choose $j$ with $1 \leq j \leq k$ maximum such that $x_{t}$ is adjacent to $p_{j}$. By $(5), k-j$ is even and $\geq 2$. Suppose that $p_{j}$ is $Y$-complete. The path $z-x_{t}-p_{j^{-}} \cdots-p_{k}$ has even length $\geq 4$, and its only $X_{t-1}$-complete vertices are its ends, and $z, x_{t}, p_{j}, X_{t-1}$ are all $Y$-complete, so by 21.1 , there is a wheel with hub $Y$ and the theorem holds. So we may assume that $p_{j}$ is not $Y$-complete. Now the path $x_{t}-p_{j}-\cdots-p_{k}$ has odd length $\geq 3$, and both its ends are $Y$-complete, and the $Y$-complete vertex $z$ has no neighbour in its interior, so by 2.2 and 2.3, an odd number of its edges are $Y$-complete. Since $p_{j}$ is not $Y$-complete, an odd number of edges of $p_{j}-\cdots-p_{k}$ are $Y$-complete. The path $z-x_{t+1}-p_{1}-\cdots-p_{k}$ (=P say) is even, by (7), and since its ends are $Y$-complete, it follows that an even number of its edges are $Y$-complete, by 2.3. We deduce that an odd number of edges of $z-x_{t+1}-p_{1}-\cdots-p_{j}$ are $Y$-complete. There is therefore a $Y$-segment $P^{\prime}$ of this path that has odd length. Since $p_{j}$ is not $Y$-complete, it follows that $P^{\prime}$ is also a $Y$-segment of $P$. If $P^{\prime}$ has length $>1$ then 21.1 applied to $P$ implies that there is a wheel with hub $Y$, and the theorem holds. So we may assume that $P^{\prime}$ has length 1. But both vertices of $P^{\prime}$ are internal vertices of $P$, since $x_{t+1}, p_{j}$ are not $Y$-complete, and again 21.1 applied to $P$ implies there is a wheel with hub $Y$. This proves 21.2.

Now we can deduce our main theorem about wheel systems, 19.1, which we restate:

21.3 Let $G \in \mathcal{F}_{8}$, let $\left(z, A_{0}\right)$ be a frame, and let $x_{0}, \ldots, x_{t+1}$ be a wheel system with hub $Y$, and with $t \geq 1$. Define $A_{i}, X_{i}$ as usual, and assume that at most one member of $Y$ has no neighbour in $A_{1}$. Suppose that for all $r$ with $1 \leq r \leq t$, if $x_{0}, x_{1}, \ldots, x_{r}, x_{t+1}$ is a wheel system, then every member of $Y$ has a neighbour in $A_{r} \cup\left\{x_{t+1}\right\}$. Then there is a wheel with hub $Y$.

Proof. Suppose there is no such wheel. Choose $r$ with $1 \leq r \leq t$, minimum such that $x_{t+1}$ has a neighbour in $A_{r}$ and a nonneighbour in $X_{r}$. By hypothesis, every member of $Y$ has a neighbour in $A_{r} \cup\left\{x_{t+1}\right\}$. By 19.2, $r>1$. Since at most one member of $Y$ has no neighbour in $A_{r-1}$ (because at most one has no neighbour in $A_{1}$ ), it follows from 21.2 that $x_{t+1}$ has a neighbour in $A_{r-1}$. Since no wheel has hub $Y, 20.1$ implies that

$$
x_{0}, \ldots, x_{r}, x_{t+1}
$$

is not a $Y$-diamond, and so $x_{t+1}$ is not $X_{r-1}$-complete. But that contradicts the minimality of $r$. Thus there is a wheel with hub $Y$. This proves 19.1 .

\section{Wheels and tails}

We continue with the proof that recalcitrant graphs do not contain wheels. Now we come to apply 19.1, as explained at the start of section 19. We use the following lemma.

22.1 Let $G \in \mathcal{F}_{8}$, not admitting a balanced skew partition, let $\left(z, A_{0}\right)$ be a frame, and let $x_{0}, \ldots, x_{s}$ be a wheel system. Let $Y \subseteq V(G) \backslash\left(A_{0} \cup\left\{z, x_{0}, \ldots, x_{s}\right\}\right)$ be nonempty and anticonnected, such that $z, x_{0}, \ldots, x_{s}$ are $Y$-complete. Then there is a sequence $x_{s+1}, \ldots, x_{t+1}$ with $t \geq s$ such that $x_{0}, \ldots, x_{t+1}$ is a wheel system with respect to the frame $\left(z, A_{0}\right)$, with hub $Y$. 
Proof. Choose a sequence $x_{s+1}, \ldots, x_{t}$, all $Y$-complete and such that $x_{0}, \ldots, x_{t}$ is a wheel system with respect to $\left(z, A_{0}\right)$, with $t$ maximum. So $t \geq s \geq 1$. Define $X_{i}$ and $A_{i}$ as usual. From 15.2 , there is a path $P$ from $z$ to $A_{t}$, disjoint from $X_{t}$ and containing no $X_{t}$-complete vertex except $z$. Let $v$ be the neighbour of $z$ in this path. From the maximality of $A_{t}$, it follows that $P$ has length 2 . So $v$ has a neighbour in $A_{t}$, and therefore $x_{0}, \ldots, x_{t}, v$ is a wheel system. From the maximality of $t$ it follows that $v$ is not $Y$-complete, and therefore $Y$ is a hub for this wheel system. This proves 22.1.

We combine 19.1 and 22.1 to prove the following.

22.2 Let $G \in \mathcal{F}_{8}$, not admitting a balanced skew partition, let $\left(z, A_{0}\right)$ be a frame, and let $x_{0}, \ldots, x_{s}$ be a wheel system. Let $Y \subseteq V(G) \backslash\left(A_{0} \cup\left\{z, x_{0}, \ldots, x_{s}\right\}\right)$ be nonempty and anticonnected, such that $z, x_{0}, \ldots, x_{s}$ are $Y$-complete. Define $A_{i}, X_{i}$ as usual, and assume that every member of $Y$ has a neighbour in $A_{s}$, and at most one member of $Y$ has no neighbour in $A_{1}$. Suppose there is no wheel with hub $Y$. Then there exists $r$ with $1 \leq r<s$, and a member $y \in Y$, and a vertex $v \notin Y \cup\left\{z, x_{0}, \ldots, x_{s}\right\}$ with the following properties:

- y has no neighbour in $A_{r} \cup\{v\}$

- $v$ is adjacent to $z$, and has a neighbour in $A_{r}$, and a non-neighbour in $X_{r}$.

Proof. By 22.1, there is a sequence $x_{s+1}, \ldots, x_{t+1}$ with $t \geq s$ such that $x_{0}, \ldots, x_{t+1}$ is a wheel system with respect to the frame $\left(z, A_{0}\right)$, with hub $Y$. By 19.1, there exists $r$ with $1 \leq r \leq t$, and a member $y \in Y$, such that $y$ has no neighbour in $A_{r} \cup\left\{x_{t+1}\right\}$, and $x_{t+1}$ has a neighbour in $A_{r}$, and a non-neighbour in $X_{r}$. Since every member of $Y$ has a neighbour in $A_{s}$, it follows that $r<s$, and the result holds (taking $v=x_{t+1}$ ). This proves 22.2 .

If $(C, Y)$ is a wheel in $G$, and there is no wheel $\left(C^{\prime}, Y^{\prime}\right)$ with $Y \subset Y^{\prime}$, we say $(C, Y)$ is an optimal wheel. Let $(C, Y)$ be a wheel in $G$. A kite for $(C, Y)$ is a vertex $y \in V(G) \backslash(Y \cup V(C))$, not $Y$-complete, that has at least four neighbours in $C$, three of which are consecutive and $Y$-complete.

22.3 Let $G \in \mathcal{F}_{8}$, not admitting a balanced skew partition, and let $(C, Y)$ be an optimal wheel in $G$. Then there is no kite for $(C, Y)$.

Proof. Assume $y$ is a kite for $(C, Y)$. Let $x_{0}-z-x_{1}$ be a subpath of $C$, all $Y$-complete and adjacent to $y$. Let $A_{0}=V(C) \backslash\left\{z, x_{0}, x_{1}\right\}$, so $x_{0}, x_{1}$ is a wheel system with respect to $\left(z, A_{0}\right)$, and $x_{0}, x_{1}$ are $Y \cup\{y\}$-complete. Thus every member of $Y \cup\{y\}$ has a neighbour in $A_{0}$, and yet there is no wheel with hub $Y \cup\{y\}$, contrary to 22.2 with $s=1$. This proves 22.3 .

Let $(C, Y)$ be a wheel in $G$, let $z \in V(C)$, and let $x_{0}, x_{1}$ be the neighbours of $z$ in $C$. A path $T$ of $G \backslash\left\{x_{0}, x_{1}\right\}$ from $z$ to $V(C) \backslash\left\{z, x_{0}, x_{1}\right\}$ is called a tail for $z$ (with respect to the wheel $(C, Y)$ ) if

- $x_{0}, z, x_{1}$ are all $Y$-complete, and there is a $Y$-complete edge in $C \backslash\left\{x_{0}, z, x_{1}\right\}$

- the neighbour of $z$ in $T$ is adjacent to $x_{0}, x_{1}$, and

- no internal vertex of $T$ is in $Y$ or is $Y$-complete.

22.4 Let $G \in \mathcal{F}_{8}$, and let $(C, Y)$ be an optimal wheel, such that no vertex is a kite for $(C, Y)$. Let $z \in V(C)$, and let $x_{0}, x_{1}$ be the neighbours of $z$ in $C$. Let $T$ be a tail for $z$, and let $y$ be the neighbour of $z$ in $T$. Let $A_{0}=V(C) \backslash\left\{z, x_{0}, x_{1}\right\}$, and let $x_{0}, \ldots, x_{t+1}$ be a wheel system with respect to the frame $\left(z, A_{0}\right)$, with hub $Y \cup\{y\}$. Define $A_{1}, \ldots, A_{t+1}$ as usual. Then $y$ has a neighbour in $A_{t} \cup\left\{x_{t+1}\right\}$. 
Proof. We assume for a contradiction that $y$ has no neighbour in $A_{t} \cup\left\{x_{t+1}\right\}$. Let $y-u_{1^{-}} \cdots-u_{n}$ be a minimal subpath of $T \backslash z$ such that $u_{n}$ has a neighbour in $A_{t}$; so $n>0$. From the maximality of $A_{t}$ it follows that $u_{n}$ is $X_{t}$-complete and therefore $X_{1}$-complete since $t \geq 1$; and since $T$ is a tail it follows that none of $u_{1}, \ldots, u_{n}$ are $Y$-complete. Let $P$ be a path with vertex set in $A_{t} \cup\left\{u_{n}\right\}$, from $u_{n}$ to some $Y$-complete vertex $p$ say, such that no vertex of $P \backslash p$ is $Y$-complete.

(1) $P$ is odd.

For $P$ has length $\geq 1$ since no vertex of $T \backslash z$ is $Y$-complete; and the only $X_{t}$-complete vertex of $P$ is $u_{n}$, and the only $Y$-complete vertex of $P$ is $p$. Since $z$ is complete to $X_{t}$ and to $Y$, and anticomplete to $V(P)$, it follows from 2.9 that $P$ has odd length. This proves (1).

Since $y, u_{1}, \ldots, u_{n-1}$ have no neighbours in $A_{t}$ it follows that $z-y-u_{1^{-}} \cdots-u_{n}-P-p$ is a path, $Q$ say.

(2) We may assume that $Q$ has even length $\geq 4$, and so $n$ is even.

For the ends of $Q$ are $Y$-complete, and since none of $y, u_{1}, \ldots, u_{n}$ are $Y$-complete, it follows that no internal vertex of $Q$ is $Y$-complete. Suppose that $Q$ has length 3 . So $n=1$, and there is an odd antipath joining $y, u_{1}$ with interior in $Y$. Hence every $Y$-complete vertex in $G$ is adjacent to one of $y, u_{1}$. In particular, since $y$ has no neighbour in $A_{t}$, it follows that $u_{1}$ is adjacent to all the $Y$-complete vertices in $C$ except $z$ (for we already showed that it is $X_{t}$-complete and therefore adjacent to $x_{0}, x_{1}$ ). Since $(C, Y)$ is not an odd wheel, it follows that $u_{1}$ is a kite for $(C, Y)$, a contradiction. So we may assume that $Q$ does not have length 3. Hence by 13.6, $Q$ has even length. From (1), it follows that $n$ is even. This proves (2).

(3) $x_{t+1}$ is adjacent to one of $u_{1}, \ldots, u_{n-1}$.

For suppose not. Choose a path $N$ from $x_{t+1}$ to $u_{n}$ with interior in $A_{t}$ (possibly of length 1 ). Then $z-y-u_{1}-\cdots-u_{n}-N-x_{t+1}-z$ is a hole, and since $n$ is even it follows that $N$ is even. Hence $z-x_{t+1}-N-u_{n}$ is an odd path; its ends are $X_{t}$-complete, its internal vertices are not, and the $X_{t}$-complete vertex $y$ has no neighbour in its interior, contrary to 2.2. This proves (3).

(4) $x_{t+1}$ is not $Y$-complete.

For suppose it is. Since $G \in \mathcal{F}_{8}$, the triple $\left(Y, X_{t+1}, Q\right)$ is not a pseudowheel. Since $y, p$ are not $X_{t+1}$-complete, it follows that no internal vertex of $Q$ is $X_{t+1}$-complete. By 2.11 applied to $Q, Y$ and $X_{t+1}$, it follows that there exists $x \in X_{t+1}$ with no neighbour in $Q \backslash z$ except possibly $p$. But $x_{t+1}$ is adjacent to one of $u_{1}, \ldots, u_{n-1}$ by (3), and all other members of $X_{t+1}$ are adjacent to $y$, a contradiction. This proves (4).

Since $x_{t+1}$ has a neighbour in $A_{t}$, there is a path $R$ from $x_{t+1}$ to some $Y$-complete vertex $r$ in $A_{t}$ with $V\left(R \backslash x_{t+1}\right) \subseteq A_{t}$ such that no vertex of $R \backslash r$ is $Y$-complete.

(5) $R$ has odd length. 
For certainly $R$ has length $\geq 1$; suppose it has length 2 , and let its middle vertex be $a$ say. There is an antipath joining $x_{t+1}, a$ with interior in $Y$, and it is odd since it can be completed to an antihole via $a-z-r-x_{t+1}$. Now $x_{t+1}, a$ are not $X_{t}$-complete (since $a \in A_{t}$ ) and so there is an antipath joining $x_{t+1}, a$ with interior in $X_{t}$, which is therefore also odd, since its union with the antipath with interior in $Y$ is an antihole. But $y$ is $X_{t}$-complete and nonadjacent to both $x_{t+1}$ and $a$ (since it has no neighbour in $A_{t}$ ), and so this antipath can be completed to an odd antihole via $a-y-x_{t+1}$, a contradiction. This proves that $R$ does not have length 2 . Hence the path $z-x_{t+1}-R-r$ does not have length 3 ; its ends are $Y$-complete and its internal vertices are not, and it has length $>1$, so by 13.6 it has even length, that is, $R$ has odd length. This proves (5).

(6) If $x_{t+1}$ is adjacent to $u_{1}$ then $u_{1}$ is $X_{t}$-complete.

For suppose not; then there is an antipath $L$ say joining $x_{t+1}, u_{1}$ with interior in $X_{t}$. So $z-u_{1}-L-x_{t+1}-y$ is an antipath of length $\geq 4$; all its internal vertices have neighbours in $A_{t} \cup\left\{u_{2}, \ldots, u_{n}\right\}$, and its ends do not. By 13.6 applied in $\bar{G}$, it has even length, and so $u_{1}-L-x_{t+1}-y$ is an odd antipath. But all its internal vertices have neighbours in $A_{t}$, and its ends do not (for $n \geq 2$ since $n$ is even), and $z$ is complete to its interior and has no neighbours in $A_{t}$, contrary to 2.2 applied in $\bar{G}$. This proves (6).

(7) None of $u_{1}, \ldots, u_{n-1}$ is $X_{t}$-complete.

For suppose that one of $u_{1}, \ldots, u_{n-1}$ is $X_{t^{-}}$complete, and let $S$ be a path from $x_{t+1}$ to some $X_{t^{-}}$ complete vertex $s$ say, with $V\left(S \backslash x_{t+1}\right) \subseteq\left\{u_{1}, \ldots, u_{n-1}\right\}$, such that $s$ is the only $X_{t}$-complete vertex in $S$. Certainly $S$ has length $\geq 1$. Suppose it has even length. Then the path $z-x_{t+1}-S-s$ is odd, and its ends are $X_{t}$-complete, and its internal vertices are not; so by 2.2 , the $X_{t}$-complete vertex $y$ has a neighbour in its interior, contrary to (6). So $S$ has odd length. The path $s-S-x_{t+1}-R-r$ therefore has even length; its only $X_{t}$-complete vertex is $s$, and its only $Y$-complete vertex is $r$, so by 13.7 , the path has length 2, that is, both $R, S$ have length 1 . Moreover, either $x_{t+1}, r$ are joined by an odd antipath with interior in $X_{t}$, or $x_{t+1}, s$ are joined by an odd antipath with interior in $Y$. The first is impossible since the antipath could be completed to an odd antihole via $r-y-x_{t+1}$, so the second holds. In particular, every $Y$-complete vertex is adjacent to one of $x_{t+1}, s$, and therefore all such vertices in $A_{t}$ are adjacent to $x_{t+1}$. In particular, $x_{t+1}$ is adjacent to all the $Y$-complete vertices in $C$ except possible $x_{0}, x_{1}$. Since there is a $Y$-complete edge in $C \backslash\left\{x_{0}, z, x_{1}\right\}$ from the definition of a tail, it follows that $x_{t+1}$ has two adjacent neighbours in $C$ of opposite wheel-parity, and at least one other neighbour in $C$; but it is not a kite, and the wheel is optimal, contrary to 16.1. This proves (7).

By (3) we may choose $i$ with $1 \leq i \leq n-1$ minimum such that $x_{t+1}$ is adjacent to $u_{i}$. By (7), the only $X_{t}$-complete vertices in the hole $z-y-u_{1^{-}} \cdots-u_{i^{-}} x_{t+1^{-}} z$ are $z, y$, and therefore by (6) this hole has length $\geq 6$. By $2.10 X_{t}$ contains a leap or a hat. If it contains a leap, there are nonadjacent vertices in $X_{t}$, joined by an odd path of length $\geq 5$ with interior in $\left\{u_{1}, \ldots, u_{i}, x_{t+1}\right\}$, and consequently with no internal vertex $Y$-complete. Since both its ends are $Y$-complete, this contradicts 13.6. So there is a hat, that is, there exists $x \in X_{t}$ with no neighbours in $\left\{u_{1}, \ldots, u_{i}, x_{t+1}\right\}$. Then $A_{t} \cup\left\{u_{1}, \ldots, u_{n}, x_{t+1}\right\}$ ( $=F$ say) catches the triangle $\{z, y, x\}$; the only neighbour of $z$ in $F$ is $x_{t+1}$; the only neighbour of $y$ in $F$ is $u_{1}$; and both $x_{t+1}, u_{1}$ are nonadjacent to $x$. Moreover $x_{t+1}$ is nonadjacent to $u_{1}$, and so $F$ contains no reflection of the triangle. This contradicts 17.1, and therefore proves 22.4. 
We combine the previous result with 19.1 to prove the following.

22.5 Let $G \in \mathcal{F}_{8}$, not admitting a balanced skew partition, and let $(C, Y)$ be an optimal wheel in $G$. Then no vertex of $C$ has a tail.

Proof. Suppose $z \in V(C)$ has a tail $T$; let $y$ be the neighbour of $z$ in $T$, and let $x_{0}, x_{1}$ be the neighbours of $z$ in $C$. Let $A_{0}=V(C) \backslash\left\{z, x_{0}, x_{1}\right\}$, so $x_{0}, x_{1}$ is a wheel system with respect to $\left(z, A_{0}\right)$, and $x_{0}, x_{1}$ are $Y \cup\{y\}$-complete. By 22.1 there exist $x_{2}, \ldots, x_{t+1}$ with $t \geq 1$ such that $x_{0}, \ldots, x_{t+1}$ is a wheel system with respect to $\left(z, A_{0}\right)$, with hub $Y \cup\{y\}$. Define $A_{i}, X_{i}$ as usual. From the construction, all members of $Y$ have a neighbour in $A_{0}$. By 19.1, there exists $r$ with $1 \leq r \leq t$, such that $x_{0}, \ldots, x_{r}, x_{t+1}$ is a wheel system and $y$ has no neighbour in $A_{r} \cup\left\{x_{t+1}\right\}$. But $Y \cup\{y\}$ is a hub for this wheel system, and $T$ is a tail for $z$. By 22.3 , there is no kite for $(C, Y)$; and so by 22.4 applied to this wheel system, $y$ has a neighbour in $A_{r} \cup\left\{x_{t+1}\right\}$, a contradiction. This proves 22.5.

\section{The end of wheels}

In this section we complete the proof that there is no wheel in a recalcitrant graph. We need the following:

23.1 Let $G \in \mathcal{F}_{8}$, not admitting a balanced skew partition, and let $(C, Y)$ be an optimal wheel in $G$. Then there is a subpath $c_{1}-c_{2}-c_{3}$ of $C$ such that $c_{1}, c_{2}, c_{3}$ are all $Y$-complete, and a path $c_{1}-p_{1}-\cdots-p_{k}-c_{3}$ such that none of $p_{1}, \ldots, p_{k}$ are in $V(C) \cup Y$, none of them is $Y$-complete, and none of them has a neighbour in $V(C) \backslash\left\{c_{1}, c_{2}, c_{3}\right\}$.

Proof. There are two nonadjacent $Y$-complete vertices in $C$ with opposite wheel-parity, say $a, b$, and by 15.2 , there is a path $P$ in $G$ joining them such that none of its interior vertices is in $Y$ or is $Y$-complete. There may be internal vertices of $P$ that belong to $C$, but we may choose a subpath $P^{\prime}$ of $P$, with ends $a^{\prime}, b^{\prime}$ say, such that $a^{\prime}, b^{\prime} \in V(C)$ have opposite wheel-parity and $P^{\prime}$ has minimum length. It follows that no vertex of the interior of $P^{\prime}$ is in $C$. Suppose $a^{\prime}, b^{\prime}$ are adjacent; then since they are in $C$ and have opposite wheel-parity, they are both $Y$-complete, and therefore neither is in the interior of $P$, and so $a, b$ are adjacent, a contradiction. So $a^{\prime}, b^{\prime}$ are nonadjacent. Let $F$ be the interior of $P^{\prime}$; then no vertex of $F$ is in $Y \cup V(C)$, no vertex of $F$ is $Y$-complete, and there are attachments of $F$ in $C$ which are nonadjacent and have opposite wheel-parity. The result follows from 22.3 and 16.2 applied to $F$. This proves 23.1 .

Now we can prove 1.8 .9 , which we restate.

23.2 Let $G \in \mathcal{F}_{8}$, not admitting a balanced skew partition; then there is no wheel in $G$. In particular, every recalcitrant graph belongs to $\mathcal{F}_{9}$.

Proof. Suppose there is a wheel in $G$, and choose an optimal wheel $(C, Y)$ such that $C$ contains as few $Y$-complete edges as possible.

(1) Exactly 4 edges of $C$ are $Y$-complete. 
For by 23.1 there is a subpath $c_{1}-c_{2}-c_{3}$ of $C$ such that $c_{1}, c_{2}, c_{3}$ are all $Y$-complete, and a path $c_{1}-p_{1}-\cdots-p_{k}-c_{3}$ such that none of $p_{1}, \ldots, p_{k}$ are in $V(C) \cup Y$, none of them is $Y$-complete, and none of them has a neighbour in $V(C) \backslash\left\{c_{1}, c_{2}, c_{3}\right\}$. Let $C^{\prime}$ be the hole formed by the union of the paths $C \backslash c_{2}, c_{1}-p_{1}-\cdots-p_{k}-c_{3}$. Then it has length $\geq 6$, and it contains fewer $Y$-complete edges than $C$. From the choice of $(C, Y)$ it follows that $\left(C^{\prime}, Y\right)$ is not a wheel, and since $C$ has at least $4 Y$-complete edges, and $C^{\prime}$ has only two fewer, it follows that exactly 4 edges of $C$ are $Y$-complete. This proves (1).

Since $(C, Y)$ is not an odd wheel, there are vertices $x_{0}, z, x_{1}, c_{1}, c_{2}, c_{3}$ of $C$, in order, and all distinct except possibly $x_{1}=c_{1}$ or $c_{3}=x_{0}$, such that the $Y$-complete edges in $C$ are $x_{0} z, z x_{1}, c_{1} c_{2}, c_{2} c_{3}$. Let $A_{0}=V(C) \backslash\left\{z, x_{0}, x_{1}\right\}$. Since $G$ does not admit a skew partition, there is a path $T$ of $G \backslash\left\{x_{0}, x_{1}\right\}$ from $z$ to $A_{0}$, such that no vertex in its interior is in $Y$ or $Y$-complete. Let $y$ be the neighbour of $z$ in $T$.

(2) $y$ is not adjacent to both $x_{0}, x_{1}$.

For assume it is. By 22.3 there is no kite for $(C, Y)$, and with respect to the wheel $(C, Y), T$ is a tail for $z$ (because at least one of the $Y$-complete edges $c_{1} c_{2}, c_{2} c_{3}$ belongs to $C \backslash\left\{x_{0}, z, x_{1}\right\}$ ). This contradicts 22.5 , and therefore proves (2).

(3) y has no neighbour in $A_{0}$.

For suppose first that it has a neighbour in $A_{0} \backslash\left\{c_{2}\right\}$, say $c$. Then $c, z$ are nonadjacent and have opposite wheel-parity in the wheel $(C, Y)$; it is not the case that $c$ and both its neighbours in $C$ are $Y$-complete, by (1) and the fact that $c \in A_{0}$; not both neighbours of $z$ in $C$ are adjacent to $y$, by (2); so 16.1 implies that $(C, Y \cup\{y\})$ is a wheel, a contradiction. So $y$ has no neighbour in $A_{0} \backslash\left\{c_{2}\right\}$. Next suppose that $y$ is adjacent to $c_{2}$. From the symmetry we may assume that $x_{0} \neq c_{3}$. Let $Q$ be the path of $C \backslash z$ between $x_{0}, c_{3}$; so $Q$ has length $>0$, and even length by 2.3. Since $x_{0}-Q-c_{3}-c_{2}-y-x_{0}$ is not an odd hole, it follows that $y$ is not adjacent to $x_{0}$. But then the hole $x_{0}-Q-c_{3}-c_{2}-y-z-x_{0}$ is the rim of an odd wheel with hub $Y$, contrary to $G \in \mathcal{F}_{8}$. So $y$ is not adjacent to $c_{2}$. This proves (3).

Let $T$ have vertices $z-y-v_{1}-\cdots-v_{n+1}$, where $v_{n+1} \in A_{0}$. From (3), $n \geq 1$. By choosing $T$ of minimum length we may assume that none of $y, v_{1}, \ldots, v_{n-1}$ have neighbours in $A_{0}$.

(4) If $n=1$ then no neighbour of $v_{1}$ in $A_{0}$ is $Y$-complete.

For otherwise we may assume $v_{2}$ is $Y$-complete. From the symmetry we may assume that $x_{0} \neq c_{3}$. Let $Q$ be the path of $C \backslash z$ between $x_{0}, c_{3}$; so $Q$ has length $>0$, and even length by 2.3. Since $y, v_{1}$ are not $Y$-complete, there is an antipath joining them with interior in $Y$, and it is odd since it can be completed to an antihole via $v_{1}-z-v_{2}-y$. Hence every $Y$-complete vertex is adjacent to one of $y, v_{1}$, and since $c_{2}, c_{3}$ are $Y$-complete and not adjacent to $y$ by (3), it follows that $v_{1}$ is adjacent to $c_{2}, c_{3}$. By (2), $v_{1}$ is adjacent to one of $x_{0}, x_{1}$, and so it has two nonadjacent neighbours in $C$, and two neighbours in $C$ of opposite wheel-parity. By 16.1, there are three consecutive vertices in $C$, all $Y$-complete and adjacent to $v_{1}$. By 22.3, $v_{1}$ has no other neighbours in $C$. Hence $x_{1}=c_{1}$ and the neighbours of $v_{1}$ in $C$ are $c_{1}, c_{2}, c_{3}$. Consequently $x_{0}$ is adjacent to $y$; but then $x_{0}-Q-c_{3}-v_{1}-y-x_{0}$ is an 
odd hole, a contradiction. This proves (4).

(5) One of $x_{0}, x_{1}$ has no neighbours in $\left\{y, v_{1}, \ldots, v_{n}\right\}$.

For let $P$ be a path $y-p_{1}-\cdots-p_{k}$ from $y$ to some $Y$-complete vertex $p_{k} \in A_{0}$, with interior in $A_{0} \cup\left\{v_{1}, \ldots, v_{n}\right\}$, such that $p_{k}$ is the only $Y$-complete vertex in $P$. Since none of $y, v_{1}, \ldots, v_{n-1}$ have neighbours in $A_{0}$ it follows that $\left\{y, v_{1}, \ldots, v_{n}\right\} \subseteq\left\{y, p_{1}, \ldots, p_{k-1}\right\}$. From (4), $k \geq 3$. Since $G \in \mathcal{F}_{8},\left(Y,\left\{x_{0}, x_{1}\right\}, z-y-p_{1}-\cdots-p_{k}\right)$ is not a pseudowheel. But the ends of the path $z-y-p_{1}-\cdots-p_{k}$ are $Y$-complete and its internal vertices are not; the path has length $\geq 4$ (and therefore has even length by 13.6); $Y, z$ are $\left\{x_{0}, x_{1}\right\}$-complete, and $y, p_{k}$ are not. So no other vertices of the path are $\left\{x_{0}, x_{1}\right\}$-complete. By 2.11, applied to the same path and the same anticonnected sets, it follows that one of $x_{0}, x_{1}$ is nonadjacent to all of $y, p_{1}, \ldots, p_{k-1}$. Since $\left\{y, v_{1}, \ldots, v_{n}\right\} \subseteq\left\{y, p_{1}, \ldots, p_{k-1}\right\}$, this proves (5).

Let $F=\left\{y, v_{1}, \ldots, v_{n}\right\}$. From the symmetry we may assume that $x_{0}$ has no neighbours in $F$. Let $S$ be a path from $y$ to $x_{0}$ with interior in $F \cup A_{0}$. It follows that $S$ has length $\geq 3$. Let $C^{\prime}$ be the hole $z-y-S-x_{0}-z$; so $C^{\prime}$ has length $\geq 6$. Suppose that $x_{0}$ is different from $c_{3}$. Since $\left(C^{\prime}, Y\right)$ is not an odd wheel, it follows that $\left(C^{\prime}, Y\right)$ is not a wheel, and so $x_{0}, z$ are the only $Y$-complete vertices in $C^{\prime}$. By 2.10, $Y$ contains a leap or a hat. A leap would imply there are two vertices in $Y$, joined by an odd path of length $\geq 5$ with interior in $F \cup A_{0}$. Hence its ends are $\left\{x_{0}, x_{1}\right\}$-complete, and its internal vertices are not, contrary to 13.6. So $Y$ contains a hat, that is, there exists $y^{\prime} \in Y$ with no neighbour in $C^{\prime}$ except $z, x_{0}$. But $F \cup A_{0}$ catches the triangle $\left\{x_{0}, y^{\prime}, z\right\}$; the only neighbour of $x_{0}$ in $F \cup A_{0}$ is its neighbour in $S$, say $s$; the only neighbour of $z$ in $F \cup A_{0}$ is $y$; and $s, y$ are nonadjacent, and both nonadjacent to $y^{\prime}$, contrary to 17.1. This proves that $x_{0}=c_{3}$, and therefore $x_{1} \neq c_{1}$. By exchanging $x_{0}, x_{1}$, we deduce that $x_{1}$ has a neighbour in $F$. There are therefore two attachments of $F$ in $C$ with opposite wheel-parity, and two that are nonadjacent. By (1), 16.2, 22.3 and the optimality of the wheel, and since $x_{0}=c_{3}$ has no neighbour in $F$, it follows that there is a path $R$ between $z, c_{2}$ with interior in $F$, and no vertex of $C$ has neighbours in the interior of $R$ except $z, c_{2}$. But then the hole formed by the union of $R$ and the path $C \backslash x_{0}$ is the rim of an odd wheel with hub $Y$, a contradiction. This proves 23.2 .

23.3 Let $G \in \mathcal{F}_{9}$, admitting no balanced skew partition, let $\left(z, A_{0}\right)$ be a frame and $x_{0}, \ldots, x_{s}$ a wheel system with respect to it, and define $X_{i}, A_{i}$ as usual. Then there is no vertex $y \in V(G) \backslash\left\{z, x_{0}, \ldots, x_{s}\right\}$ that is $\left\{z, x_{0}, \ldots, x_{s}\right\}$-complete and has a neighbour in $A_{s}$.

Proof. Suppose there is such a frame, wheel system, and $y$, and choose them with $s$ minimum (it is important here that we minimize over all choices of the frame, not just of the wheel system); say $\left(z, A_{0}\right), x_{0}, \ldots, x_{s}$ and $y$ respectively. By 22.2 , there exists $r$ with $1 \leq r<s$, and a vertex $v$ such that $y$ has no neighbour in $A_{r} \cup\{v\}$, and $v$ is adjacent to $z$, and has a neighbour in $A_{r}$, and a non-neighbour in $X_{r}$. Then $\left(y, A_{0}\right)$ is a frame, and $x_{0}, \ldots, x_{r}$ is a wheel system with respect to it, and $z$ is $\left\{y, x_{0}, \ldots, x_{r}\right\}$-complete, and has a neighbour in $A_{r}^{\prime}$ (namely $v$ ), where $A_{r}^{\prime}$ is the maximal connected subset of $V(G)$ including $A_{0}$ and containing no neighbour of $y$ and no $X_{r}$-complete vertex. But this contradicts the minimality of $s$. This proves 23.3.

Now we can prove 1.8.10, the following. 
23.4 Let $G \in \mathcal{F}_{9}$, admitting no balanced skew partition, and let $C$ be a hole in $G$ of length $\geq 6$. Then there is no vertex of $G \backslash V(C)$ with three consecutive neighbours in $C$. In particular, every recalcitrant graph belongs to $\mathcal{F}_{10}$.

Proof. Suppose that there is such a vertex, say $y$, and let it be adjacent to $x_{0}, z, x_{1} \in V(C)$, where $x_{0}-z-x_{1}$ is a path. Let $A_{0}=V(C) \backslash\left\{z, x_{0}, x_{1}\right\}$. By 23.3 applied to $\left(z, A_{0}\right)$ and $x_{0}, x_{1}$, it follows that $y$ has no other neighbour in $C$. Choose $t$ maximum such that there is a sequence $x_{2}, \ldots, x_{t}$ with the following properties:

- for $2 \leq i \leq t$, there is a connected subset $A_{i-1}$ of $V(G)$ including $A_{i-2}$, containing a neighbour of $x_{i}$, containing no neighbour of $z$ or $y$, and containing no $\left\{x_{0}, \ldots, x_{i-1}\right\}$-complete vertex,

- for $1 \leq i \leq t, x_{i}$ is not $\left\{x_{0}, \ldots, x_{i-1}\right\}$-complete, and

- $x_{0}, \ldots, x_{t}$ are $\{y, z\}$-complete.

Since $G$ admits no skew partition by 15.1 , there is a path $P$ from $\{z, y\}$ to $A_{0}$, disjoint from $\left\{x_{0}, \ldots, x_{t}\right\}$ and containing no $\left\{x_{0}, \ldots, x_{t}\right\}$-complete vertex in its interior. Choose such a path of minimum length. From the symmetry between $z, y$ we may assume its first vertex is $y$; say the path is $y-p_{1}-\cdots-p_{k+1}$, where $p_{k+1} \in A_{0}$. From the minimality of the length of $P$ it follows that $z$ is not adjacent to any of $p_{2}, \ldots, p_{k}$. If $z$ is adjacent to $p_{1}$ then we may set $x_{t+1}=p_{1}$, contrary to the maximality of $t$. So $p_{1}, \ldots, p_{k+1}$ are all nonadjacent to $z$. Hence $\left(z, A_{0}\right)$ is a frame, and $x_{0}, \ldots, x_{t}$ is a wheel system with respect to it, and $y$ is adjacent to all of $z, x_{0}, \ldots, x_{t}$, and there is a connected subset of $V(G)$ including $A_{0}$, containing a neighbour of $y$, containing no neighbour of $z$, and containing no $\left\{x_{0}, \ldots, x_{t}\right\}$-complete vertex. But this contradicts 23.3. This proves 23.4.

This has the following useful corollary, which is 1.8.11.

23.5 Let $G \in \mathcal{F}_{10}$; then $G$ does not contain both a hole of length $\geq 6$ and an antihole of length $\geq 6$. In particular, for every recalcitrant graph $G$, one of $G, \bar{G}$ belongs to $\mathcal{F}_{11}$.

Proof. Let $C$ be a hole and $D$ an antihole, both of length $\geq 6$. Let $W=V(C) \cap V(D), A=V(C) \backslash W$, and $B=V(D) \backslash W$. Let $W, A, B$ have cardinality $w, a, b$ respectively. Let there be $p$ edges between $A$ and $W, q$ edges between $B$ and $W, r$ edges between $A$ and $B$, and $s$ edges with both ends in $W$. Let there be $p^{\prime}$ nonedges between $A$ and $W, q^{\prime}$ nonedges between $B$ and $W, r^{\prime}$ nonedges between $A$ and $B$, and $s^{\prime}$ nonedges with both ends in $W$. By 2.3, and since $G \in \mathcal{F}_{10}$, every vertex in $B$ has at most $\frac{1}{2}(a+w)$ neighbours in $C$, so $q+r \leq \frac{1}{2}(a+w) b$. Also, every vertex in $W$ has at most two neighbours in $A \cup W$, so $p+2 s \leq 2 w$. Summing, we obtain

$$
p+q+r+2 s \leq \frac{1}{2} a b+\frac{1}{2} b w+2 w .
$$

By the same argument in the complement we deduce that

$$
p^{\prime}+q^{\prime}+r^{\prime}+2 s^{\prime} \leq \frac{1}{2} a b+\frac{1}{2} a w+2 w .
$$

But

$$
p+p^{\prime}+q+q^{\prime}+r+r^{\prime}+2 s+2 s^{\prime}=a b+a w+b w+w(w-1)
$$




$$
4 w \geq \frac{1}{2} a w+\frac{1}{2} b w+w(w-1)
$$

that is,

$$
w(a+b+2 w-10) \leq 0 .
$$

Since $a+w, b+w \geq 6$, it follows that $w=0$, and so $C, D$ are disjoint. Moreover, equality holds throughout this calculation, so every vertex in $D$ is adjacent to exactly half the vertices of $C$ and vice versa. By 2.3 , and since $G \in \mathcal{F}_{10}$, it follows that for each $v \in D$, its neighbours in $C$ are pairwise nonadjacent. Let $C$ have vertices $c_{1}, \ldots, c_{m}$ in order, and let $D$ have vertices $d_{1}, \ldots, d_{n}$. So for every vertex of $D$, its set of neighbours in $V(C)$ is either the set of all $c_{i}$ with $i$ even, or the set with $i$ odd, and the same with $C, D$ exchanged. We may assume that $c_{1}$ is adjacent to $d_{1}$. Hence the edges between $\left\{c_{1}, c_{2}, c_{4}, c_{5}\right\}$ and $\left\{d_{1}, d_{2}, d_{4}, d_{5}\right\}$ are $c_{1} d_{1}, c_{1} d_{5}, c_{2} d_{2}, c_{2} d_{4}, c_{4} d_{2}, c_{4} d_{4}, c_{5} d_{1}, c_{5} d_{5}$; and so the subgraph induced on these eight vertices is a double diamond, contrary to $G \in \mathcal{F}_{10}$. This proves 23.5 .

Let us mention a theorem of [12], which could be applied at this stage as an alternative to the next section, the following (and see also [8] for some related material):

23.6 Let $G \in \mathcal{F}_{5}$. Suppose that for every hole $C$ in $G$ of length $\geq 6$, and every vertex $v \in V(G) \backslash$ $V(C)$, either:

- $v$ has $\leq 3$ neighbours in $C$, or

- $v$ has exactly 4 neighbours in $C$, say $a, b, c, d$, where $a b$ and $c d$ are edges, or

- $v$ is $V(C)$-complete, or

- no two neighbours of $v$ in $C$ are adjacent.

Suppose also that the same holds in $\bar{G}$. Then either one of $G, \bar{G}$ is bipartite or a line graph of a bipartite graph, or $G$ admits a loose skew partition.

The method we give below is somewhat shorter than the proof of 23.6 in [12], however.

\section{The end}

We recall that we are trying to prove 13.5. In view of 23.5, it suffices to show the following, which is 1.8.12, and the objective of the remainder of the paper:

24.1 Let $G \in \mathcal{F}_{11}$; then either $G$ is complete, or $G$ is bipartite, or $G$ admits a balanced skew partition.

We begin with a further strengthening of 13.6, as follows.

24.2 Let $G \in \mathcal{F}_{11}$, and let $P$ be a path in $G$ with odd length. Let $X \subseteq V(G)$ be anticonnected, such that both ends of $P$ are $X$-complete. Then some edge of $P$ is $X$-complete.

Proof. Suppose not; then from 13.6, $P$ has length 3 (let its vertices be $p_{1}, p_{2}, p_{3}, p_{4}$ in order) and $p_{2}, p_{3}$ are joined by an antipath $Q$ with interior in $X$. But then $p_{2}-Q-p_{3}-p_{1}-p_{4}-p_{2}$ is an antihole of length $>4$, a contradiction. This proves 24.2 . 
24.3 Let $G \in \mathcal{F}_{11}$. Let $X \subseteq V(G)$ be nonempty and anticonnected, and let $p_{1}-\cdots-p_{n}$ be a path of $G \backslash X$ with $n \geq 4$, such that $p_{1}, p_{n}$ are $X$-complete and $p_{2}, \ldots, p_{n-1}$ are not. There is no vertex $y \in V(G) \backslash\left(X \cup\left\{p_{1}, \ldots, p_{n}\right\}\right)$ such that $y$ is $X$-complete and adjacent to $p_{1}, p_{2}$.

Proof. Suppose such a vertex $y$ exists. By 24.2, $n$ is odd, and therefore $n \geq 5$. Let $Q$ be an antipath joining $p_{2}, p_{3}$ with interior in $X$. Since $Q$ can be completed to an antihole via $p_{3}-p_{n}-p_{2}$, it follows that $Q$ has length 2 , and so there exists $x \in X$ nonadjacent to $p_{2}, p_{3}$. Since $x$ is adjacent to $p_{n}$, we may choose $i$ with $2 \leq i \leq n$ minimum such that $x$ is adjacent to $p_{i}$. Hence $x-p_{1}-\cdots-p_{i}-x$ is a hole of length $\geq 6$, and $y$ has three consecutive neighbours in it, contrary to $G \in \mathcal{F}_{11}$. This proves 24.3.

The next is a strengthening of 17.1 .

24.4 Let $G \in \mathcal{F}_{11}$. Let $X_{1}, X_{2}, X_{3}$ be disjoint nonempty anticonnected sets, complete to each other. Let $F \subseteq V(G) \backslash\left(X_{1} \cup X_{2} \cup X_{3}\right)$ be connected, such that for $i=1,2,3$ there is an $X_{i}$-complete vertex in $F$. Then there is a vertex in $F$ complete to two of $X_{1}, X_{2}, X_{3}$.

Proof. Suppose not; then we may assume $F$ is minimal with this property.

(1) If $p_{1}, \ldots, p_{n}$ is a path in $F$, and $p_{1}$ is its unique $X_{1}$-complete vertex and $p_{n}$ is its unique $X_{2}$ complete vertex then $n$ is even.

For $n>1$, since no vertex is both $X_{1}$-complete and $X_{2}$-complete. Assume $n$ is odd; then by 13.7, $n=3$. But there is an antipath $Q_{1}$ between $p_{2}, p_{3}$ with interior in $X_{1}$, and an antipath $Q_{2}$ between $p_{1}, p_{2}$ with interior in $X_{2}$; and then $p_{2}-Q_{1}-p_{3}-p_{1}-Q_{2}-p_{2}$ is an antihole of length $>4$, a contradiction. This proves (1).

From the minimality of $F$, there are (up to symmetry) three cases:

1. For $i=1,2,3$ there is a unique $X_{i}$-complete vertex $v_{i} \in F$; there is a vertex $u \in F$ different from $v_{1}, v_{2}, v_{3}$, and three paths $P_{1}, P_{2}, P_{3}$ in $F$, all of length $\geq 1$, such that each $P_{i}$ is from $v_{i}$ to $u$, and for $1 \leq i<j \leq 3, V\left(P_{i} \backslash u\right)$ is disjoint from $V\left(P_{j} \backslash u\right)$ and there is no edge between them.

2. For $i=1,2,3$ there is a unique $X_{i}$-complete vertex $v_{i} \in F$; there are three paths $P_{1}, P_{2}, P_{3}$ in $F$, where each $P_{i}$ is from $v_{i}$ to some $u_{i}$ say, possibly of length 0 ; and for $1 \leq i<j \leq 3, V\left(P_{i}\right)$ is disjoint from $V\left(P_{j}\right)$ and the only edge between $V\left(P_{i}\right), V\left(P_{j}\right)$ is $u_{i} u_{j}$.

3. For $i=1,2$ there is a unique $X_{i}$-complete vertex $v_{i} \in F$, and there is a path $P$ in $F$ between $v_{1}, v_{2}$ containing at least one $X_{3}$-complete vertex.

Suppose that the first holds, and let $P_{1}, P_{2}, P_{3}$ be as in the first case. Then some two of $P_{1}, P_{2}, P_{3}$ have lengths of the same parity, and their union violates (1).

Now suppose the second holds, and for $i=1,2,3$ let $u_{i}, v_{i}, P_{i}$ be as in the second case. Let $Q_{1}$ be an antipath joining $u_{2}, u_{3}$ with interior in $X_{1}$, and define $Q_{2}, Q_{3}$ similarly. If $P_{1}, P_{2}, P_{3}$ all have length 0 , then the union of $Q_{1}, Q_{2}, Q_{3}$ is an antihole of length $>4$, a contradiction. So we may assume that $P_{1}$ has length $>0$, and hence $u_{1} \neq v_{1}$. Since $v_{1}-u_{2}-Q_{1}-u_{3}-v_{1}$ is an antihole, $Q_{1}$ has length 1 . Since $u_{1}, u_{3}$ are not $X_{1}$-complete, they are joined by an antipath with interior in $X_{1}$, and 
its union with $Q_{2}$ is an antihole; so $Q_{2}$ has length 2 , and similarly so does $Q_{3}$. For $i=1,2,3$ let $x_{i}$ be the middle vertex of $Q_{i}$. So

$$
V\left(P_{1} \backslash u_{1}\right) \cup V\left(P_{2} \backslash u_{2}\right) \cup V\left(P_{3} \backslash u_{3}\right) \cup\left\{x_{1}, x_{2}, x_{3}\right\}
$$

is connected, and catches the triangle $\left\{u_{1}, u_{2}, u_{3}\right\}$; and none of its vertices have two neighbours in the triangle, and it contains no reflection of the triangle since there is no antihole of length 6 . This is contrary to 17.1 .

Now suppose the third holds, and let $v_{1}, v_{2}, P$ be as in the third case. Let $P$ have vertices $p_{1}, \ldots, p_{n}$ where $v_{1}=p_{1}$ and $v_{2}=p_{n}$. Since one of its vertices is $X_{3}$-complete and $p_{1}, p_{n}$ are not, it follows that $n \geq 3$; and by (1), $n$ is odd, so $n \geq 4$. Choose $i$ minimum and $j$ maximum with $1 \leq i, j \leq n$ such that $p_{i}, p_{j}$ are $X_{3}$-complete. So $i>1$, and $i$ is even by (1), and similarly $j<n$ and $j$ is odd. So the path $p_{i^{-}} \cdots-p_{j}$ has odd length, and so by 24.2 one of its edges is $X_{3}$-complete, say $p_{k} p_{k+1}$ where $2 \leq k \leq n-2$. Now $p_{k}, p_{k+1}$ are joined by an antipath with interior in $X_{1}$, and by another with interior in $X_{2}$, and the union of these is an antihole; so they both have length 2. Hence for $i=1,2$ there exist $x_{i} \in X_{i}$ nonadjacent to both $p_{k}, p_{k+1}$. Let $R$ be a path between $p_{k+2}, p_{k-1}$ with interior in $\left(V(P) \backslash\left\{p_{k}, p_{k+1}\right\}\right) \cup\left\{x_{1}, x_{2}\right\}$. Then $R$ can be completed to a hole $C$ via $p_{k-1}-p_{k}-p_{k+1}-p_{k+2}$, and $C$ has length $\geq 6$, and at least one edge of $C$ is $X_{3}$-complete, namely $p_{k} p_{k+1}$, and at least one more vertex of it is $X_{3}$-complete, since $R$ uses at least one of $x_{1}, x_{2}$. But this contradicts 2.3 , and the hypothesis that $G \in \mathcal{F}_{11}$.

This proves 24.4 .

24.5 Let $G \in \mathcal{F}_{11}$, admitting no balanced skew partition. Let $X, Y$ be disjoint anticonnected subsets of $V(G)$, complete to each other, and let $p_{1}-\cdots-p_{n}$ be a path of $G \backslash(X \cup Y)$, with $n \geq 2$, such that $p_{1}$ is the unique $X$-complete vertex in the path, and $p_{n}$ is the unique $Y$-complete vertex. Then there is no $z \in V(G) \backslash\left(X \cup Y \cup\left\{p_{1}, \ldots, p_{n}\right\}\right)$, complete to $X \cup Y$ and nonadjacent to $p_{1}, p_{n}$.

Proof. Suppose that $z$ exists, and choose $X$ maximal. By 15.2, there is a path $Q$ in $G$ from $z$ to $p_{1}$, such that none of its internal vertices is in $X$ or is $X$-complete. Since no vertex of $\left\{p_{2}, \ldots, p_{n}\right\}$ is $X$ complete, we may choose $Q$ such that if $z$ has a neighbour in $\left\{p_{2}, \ldots, p_{n}\right\}$ then $V(Q) \subseteq\left\{z, p_{1}, \ldots, p_{n}\right\}$. The connected subset $V(Q \backslash z) \cup\left\{p_{1}, \ldots, p_{n}\right\}$ (=F say) contains an $X$-complete vertex, a $Y$-complete vertex, and a $\{z\}$-complete vertex. The only $X$-complete vertex in $F$ is $p_{1}$, and that is not $Y$-complete or $\{z\}$-complete; so by 24.4 some vertex in $F$ is $Y$-complete and adjacent to $z$. If $z$ has a neighbour in $\left\{p_{1}, \ldots, p_{n}\right\}$, then $V(Q) \subseteq\left\{z, p_{1}, \ldots, p_{n}\right\}$, and so $p_{n}$ is the only vertex of $F$ that is $Y$-complete; and it is not adjacent to $z$, a contradiction. So $z$ has no neighbour in $\left\{p_{1}, \ldots, p_{n}\right\}$, and therefore only one vertex in $F$ is adjacent to $z$, the neighbour of $z$ in $Q$, say $q$. Hence $q$ is nonadjacent to $p_{1}$, for otherwise we could add $q$ to $X$, contrary to the maximality of $X$. Consequently $Q$ has length $>2$. This contradicts 24.3 applied to $Q, X$ and any vertex $y \in Y$. This proves 24.5.

We deduce

24.6 Let $G \in \mathcal{F}_{11}$, admitting no balanced skew partition, and let $C$ be a hole. If $z \in V(G) \backslash V(C)$ has two neighbours in $C$ that are adjacent, then $C$ has length 4 and $z$ has a third neighbour in $C$. In particular, $G$ has no antipath of length 4 .

Proof. Let $C$ be the hole with vertices $p_{1}, \ldots, p_{n+2}$ in order, and assume some $z \in V(G) \backslash V(C)$ is adjacent to $p_{n+1}, p_{n+2}$. By 24.5 , taking $X=\left\{p_{n+1}\right\}$ and $Y=\left\{p_{n+2}\right\}$ we deduce that $z$ is adjacent to at least one of $p_{1}, p_{n}$. Since $G \in \mathcal{F}_{11}$ it follows that $C$ has length 4. This proves 24.6. 
24.7 Let $G \in \mathcal{F}_{11}$, admitting no balanced skew partition. Let $X_{1}, X_{2}, X_{3}$ be pairwise disjoint, nonempty, anticonnected subsets of $V(G)$, complete to each other. Let $F \subseteq V(G) \backslash\left(X_{1} \cup X_{2} \cup X_{3}\right)$ be connected, such that for at least two values of $i \in\{1,2,3\}$, every member of $X_{i}$ has a neighbour in $F$. Then some vertex of $F$ is complete to two of $X_{1}, X_{2}, X_{3}$.

Proof. Assume not, and choose a counterexample with $X_{1} \cup X_{2} \cup X_{3} \cup F$ minimal. Suppose $F$ contains an $X_{i}$-complete vertex for two values of $i \in\{1,2,3\}$, say $i=1,2$; and choose a path $p_{1}-\cdots-p_{n}$ of $F$ such that $p_{1}$ is $X_{1}$-complete and $p_{n}$ is $X_{2}$-complete, with $n$ minimum. So $n \geq 2$. From the minimality of $F, F=V(P)$, and there is a vertex $x_{1} \in X_{1}$ such that $p_{1}$ is its only neighbour in $F$, and there exists $x_{2} \in X_{2}$ such that $p_{n}$ is its only neighbour in $F$. By 24.6 applied to the hole $x_{2}-x_{1}-p_{1}-\cdots-p_{n}-x_{2}$ and any $x_{3} \in X_{3}$, it follows that $n=2$. Let $Q$ be an antipath between $p_{1}, p_{2}$ with interior in $X_{3}$; since $p_{1}$ has a nonneighbour $x_{2} \in X_{2}$, and $p_{2}$ has a nonneighbour $x_{1} \in X_{1}$, it follows that $x_{1}-p_{2}-Q-p_{1}-x_{2}$ is an antipath of length $\geq 5$, contrary to 24.5 .

So there is at most one $i$ such that $F$ contains $X_{i}$-complete vertices, and from the symmetry we may assume that $F$ contains no $X_{1}$ - or $X_{2}$-complete vertices. We may also assume that all members of $X_{1}$ have neighbours in $F$, and therefore $\left|X_{1}\right| \geq 2$; choose distinct $x_{1}, x_{1}^{\prime} \in X_{1}$ such that $X_{1} \backslash\left\{x_{1}\right\}, X_{1} \backslash\left\{x_{1}^{\prime}\right\}$ are both anticonnected. From the minimality of $X_{1}$, there is a vertex $f$ of $F$ complete to two of $X_{1} \backslash\left\{x_{1}\right\}, X_{2}, X_{3}$, and therefore complete to $X_{1} \backslash\left\{x_{1}\right\}$ and $X_{3}$, and similarly a vertex $f^{\prime}$ of $F$ complete to $X_{1} \backslash\left\{x_{1}^{\prime}\right\}$ and $X_{3}$. Let $P$ be a path in $F$ between $f, f^{\prime}$. Since all vertices of $X_{1} \cup X_{3}$ have neighbours in $V(P)$, the minimality of $F$ implies that $F=V(P)$; and moreover, since all vertices of $\left(X_{1} \backslash\left\{x_{1}\right\}\right) \cup X_{3}$ are adjacent to $f$, the minimality of $F$ implies that $f^{\prime}$ is the unique neighbour of $x_{1}$ in $F$. Similarly $f$ is the unique neighbour of $x_{1}^{\prime}$ in $F$. Let $Q$ be an antipath in $X_{1}$ joining $x_{1}, x_{1}^{\prime}$. Since $f$ has a nonneighbour $x \in X_{2}, x-f-x_{1}-Q-x_{1}^{\prime}$ is an antipath, and so $Q$ has length 1 , and hence $x_{1}, x_{1}^{\prime}$ are nonadjacent. From the minimality of $F$, there exists $x_{2} \in X_{2}$ with no neighbour in $F \backslash\{f\}$. If $x_{2}$ is also nonadjacent to $f$, then $x_{2}-x_{1}-f^{\prime}-P-f-x_{1}^{\prime}-x_{2}$ is a hole of length $\geq 6$, and any member of $X_{3}$ has three consecutive neighbours on it, contrary to $G \in \mathcal{F}_{11}$. But then $x_{1}$ has two consecutive neighbours on the hole $x_{1}^{\prime}-f-P-f^{\prime}-x_{1}-x_{2}-x_{1}^{\prime}$, and this hole has length $>4$, contrary to 24.6. This proves 24.7.

Now we can complete the proof of 24.1, and hence of 13.5 and therefore of 1.3 and 1.2 , as follows. Proof of 24.1. Let $G \in \mathcal{F}_{11}$, admitting no balanced skew partition. We may assume that $G$ is not bipartite, and therefore has a triangle. Consequently we may choose disjoint nonempty anticonnected sets $X_{1}, \ldots, X_{k}$, complete to each other, with $k \geq 3$, with maximal union. Suppose first that $X_{1} \cup \cdots \cup X_{k} \neq V(G)$, and let $F=V(G) \backslash\left(X_{1} \cup \cdots \cup X_{k}\right)$. By $15.2\left(\right.$ applied to $X_{k}$ and $\left.X_{1} \cup \cdots \cup X_{k-1}\right)$, $F$ is connected and every vertex of $X_{1} \cup X_{2}$ has a neighbour in it. By 24.7, some vertex $v \in F$ is complete to two of $X_{1}, X_{2}, X_{3}$. We may assume that for some $i$ with $2 \leq i \leq k, v$ is $X_{j}$-complete for $1 \leq j \leq i$ and not $X_{j}$-complete for $i<j \leq k$. Define

$$
X_{i+1}^{\prime}=X_{i+1} \cup \cdots \cup X_{k} \cup\{v\} ;
$$

then the sets $X_{1}, \ldots, X_{i}, X_{i+1}^{\prime}$ violate the optimality of the choice of $X_{1}, \ldots, X_{k}$.

Hence $X_{1} \cup \cdots \cup X_{k}=V(G)$, and therefore $\bar{G}$ has at least three components. From 15.2 it follows that $G$ is complete. This proves 24.1. 


\section{Acknowledgements}

We worked on pieces of this with several other people, and we would particularly like to thank Jim Geelen, Bruce Reed, Chunwei Song and Carsten Thomassen for their help.

We would also like to acknowledge our debt to Michele Conforti, Gérard Cornuéjols and Kristina Vušković; their pioneering work was very important to us, and several of their ideas were seminal for this paper. In particular, they conjectured the truth of 1.3 (or something quite close to it), and suggested that Berge graphs containing wheels should have skew partitions induced by the wheels, and Vušković convinced us that prisms were interesting, and a theorem like 13.4 should exist. In addition, they proved a sequence of steadily improving theorems, saying that any counterexample to the strong perfect graph conjecture must contain subgraphs of certain kinds (wheels and parachutes), and those gave us a great incentive to work from the other end, proving that minimum counterexamples cannot contain subgraphs of certain kinds.

Finally, we would like to acknowledge the American Institute of Mathematics, who generously supported two of us full-time for six months to work on this project.

\section{References}

[1] C. Berge, "Färbung von Graphen, deren sämtliche bzw. deren ungerade Kreise starr sind", Wiss. Z. Martin-Luther-Univ. Halle-Wittenberg Math.-Natur. Reihe 10 (1961), 114.

[2] M. E. Bertschi and B. A. Reed, "A note on even pairs", Discrete Math. 65 (1987), 317-318.

[3] M. Chudnovsky, "Berge trigraphs and their applications", PhD thesis, Princeton University, 2003.

[4] M. Chudnovsky, "Berge trigraphs", manuscript, March 2004, submitted for publication.

[5] M. Chudnovsky, N. Robertson, P. Seymour and R. Thomas, "Progress on perfect graphs", Math. Programming, Ser. B, 97 (2003), 405-422.

[6] V. Chvátal, "Star-cutsets and perfect graphs", J. Combinatorial Theory, Ser. B 39 (1985), 189-199.

[7] V. Chvátal and N. Sbihi, "Bull-free Berge graphs are perfect", Graphs and Combinatorics 3 (1987), 127-139.

[8] M. Conforti and G. Cornuéjols, "Graphs without odd holes, parachutes or proper wheels: a generalization of Meyniel graphs and of line graphs of bipartite graphs", J. Combinatorial Theory, Ser. B, 87 (2003), 300-330.

[9] M. Conforti, G. Cornuéjols and M. R. Rao, "Decomposition of balanced matrices", J. Combinatorial Theory, Ser. B, 77 (1999), 292-406.

[10] M. Conforti, G. Cornuéjols and K. Vušković, "Square-free perfect graphs", J. Combinatorial Theory, Ser. B, 90 (2004), 257-307. 
[11] M. Conforti, G. Cornuéjols, K. Vušković and G. Zambelli, "Decomposing Berge graphs containing proper wheels", manuscript, March 2002.

[12] M. Conforti, G. Cornuéjols and G. Zambelli, "Decomposing Berge graphs containing no proper wheels, long prisms or their complements", Combinatorica, to appear.

[13] G. Cornuéjols and W.H. Cunningham, "Compositions for perfect graphs", Discrete Math. 55 (1985), 245-254.

[14] C. T. Hoang, "Some properties of minimal imperfect graphs", Discrete Math. 160 (1996), 165175 .

[15] D. König, "Über Graphen und ihre Anwendung auf Determinantentheorie und Mengenlehre", Math. Ann. 77 (1916), 453-465.

[16] L. Lovász, "A characterization of perfect graphs", J. Combinatorial Theory, Ser. B 13 (1972), 95-98.

[17] W. McCuaig, "Pólya's permanent problem", preprint, 1996.

[18] H. Meyniel, “A new property of critical imperfect graphs and some consequences", European J. Combinatorics 8 (1987), 313-316.

[19] B. A. Reed, "A semistrong perfect graph theorem", PhD thesis, McGill Univ., 1986.

[20] J. Ramirez-Alfonsin and B. Reed (eds.), Perfect Graphs, Wiley, Chichester, 2001.

[21] N. Robertson, P. Seymour and R. Thomas, "Permanents, Pfaffian orientations, and even directed circuits", Annals of Math., 150 (1999), 929-975.

[22] N. Robertson, P. Seymour and R. Thomas, "Tutte's edge-colouring conjecture", J. Combinatorial Theory, Ser. B 70 (1997), 166 - 183.

[23] F. Roussel and P. Rubio, "About skew partitions in minimal imperfect graphs", J. Combinatorial Theory, Ser. B 83 (2001), 171-190.

[24] P. Seymour, "Decomposition of regular matroids", J. Combinatorial Theory, Ser. B 28 (1980), 305-359.

[25] K. Wagner, "Über eine Eigenschaft der ebenen Komplexe", Math. Ann. 114 (1937), 570-590. 